\title{
VALIDATION OF A CFD APPROACH FOR GAS TURBINE INTERNAL COOLING PASSAGE HEAT TRANSFER PREDICTION
}

\author{
A Thesis \\ presented to \\ the Faculty of California Polytechnic State University, \\ San Luis Obispo
}

\author{
In Partial Fulfillment \\ of the Requirements for the Degree \\ Master of Science in Aerospace Engineering
}

by

Daniel Wilde

June 2015 
(C)2015

Daniel Wilde

ALL RIGHTS RESERVED 


\section{COMMITTEE MEMBERSHIP}

TITLE:

AUTHOR:

DATE SUBMITTED:

COMMITTEE CHAIR:

COMMITTEE MEMBER

COMMITTEE MEMBER:

Validation of A CFD Approach for Gas Turbine Internal Cooling Passage Heat Transfer Prediction

Daniel Wilde

June 2015

David Marshall, Ph.D.

Professor of Aerospace Engineering

Robert McDonald, Ph.D.

Associate Professor of Aerospace Engineering

Paul Choboter, Ph.D.

Associate Professor of Mathematics

COMMITTEE MEMBER: $\quad$ Kim Shollenberger, Ph.D.

Professor of Mechanical Engineering 


\begin{abstract}
Validation of a CFD Approach for Gas Turbine Internal Cooling Passage Heat Transfer Prediction

Daniel Wilde
\end{abstract}

GE Power \& Water, has directed the enclosed research regarding CFD and its' application to internal convective turbine cooling. This report was prepared as an account of work sponsored by an agency of the United States Government. Neither the United States Government nor any agency thereof, nor any of their employees, makes any warranty, express or implied, or assumes any legal liability or responsibility for the accuracy, completeness, or usefulness of any information, apparatus, product, or process disclosed, or represents that its use would not infringe privately owned rights. Reference herein to any specific commercial product, process, or service by trade name, trademark, manufacturer, or otherwise does not necessarily constitute or imply its endorsement, recommendation, or favoring by the United States Government or any agency thereof. The views and opinions of authors expressed herein do not necessarily state or reflect those of the United States Government or any agency thereof.

This report describes the development and application of a validated Computational Fluid Dynamics (CFD) modelling approach for internal cooling passages in rotating turbomachinery. A CFD Modelling approach and accompanying assumptions are tuned and validated against academically available experimental results for various serpentine passages. Criteria of the CFD modelling approach selected for investigation into advanced internal cooling flows include accuracy, robustness, industry familiarity, and computational cost.

Experimental data from NASA HOST (HOt Section Technology), Texas A\&M, and University of Manchester tests are compared to RANS CFD results generated using Fluent v14.5 in order to benchmark a CFD modelling approach.

Capability of various turbulence models in the representation of cooling physics is evaluated against experimental data. Model sensitivity to boundary conditions and mesh density is also evaluated.

The development of a validated computational model of internal turbine cooling channels with bounded error allows for the identification of particular shortcomings of heat transfer correlations and provides a baseline for future CFD based exploration of internal turbine cooling concepts. 


\section{ACKNOWLEDGMENTS}

The Master's Thesis is considered a culminating experience for graduate education and is the product of instruction, collaboration, and support which have come from many important figures around me who I would like to thank.

I would like to thank my advisor, Dr. David Marshall for taking me on as a graduate student and remaining supportive and available over the long duration of the thesis process, in spite of my having moved out of state.

Additionally, I would like to thank my team at QuEST, Jean Valcin, Daniel Sartoris, and Jan Zlebek, who not only provided feedback on the engineering analysis behind this project, but also helped with data processing, formatting, and generation of many plots and images which appear in this report.

I would also like to thank GE Power \& Water for directing this research and providing the opportunity to perform some truly interesting analysis. Specifically, I would like to thank the GE engineers and managers involved in the project for attending my weekly updates and providing feedback and insight. Thanks to Benjamin Lacy, Fred Willet, Sandip Dutta, Franklin Fu, and Sam Ciray

I would also like to thank my parents who have been there for me me all along the way.

Finally, I would like to thank my wife, Jordan Elkins who has remained supportive throughout this entire process, in spite of it taking years longer than I had expected. Thanks for keeping me focused most of the time. 


\section{TABLE OF CONTENTS}

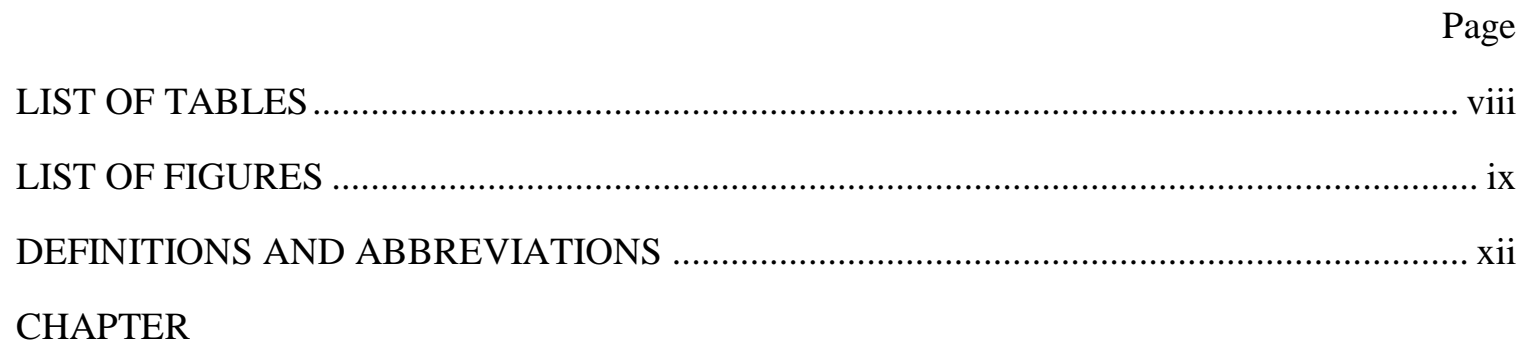

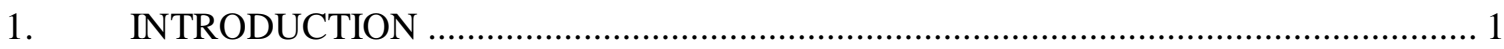

1.1 Turbine Cooling and Serpentine Passages................................................................ 1

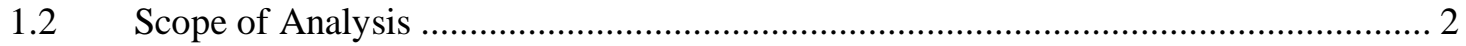

2. PHYSICAL PHENOMENA OF INTERNAL COOLING .............................................. 4

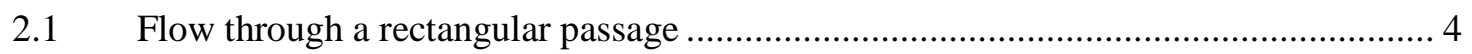

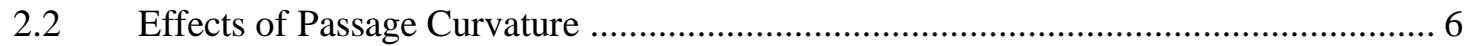

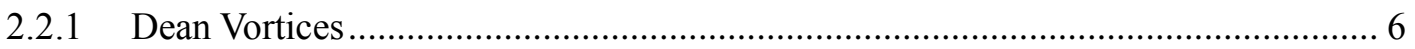

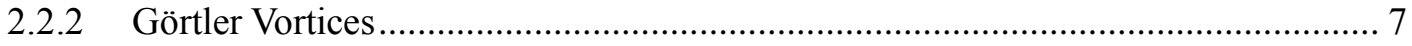

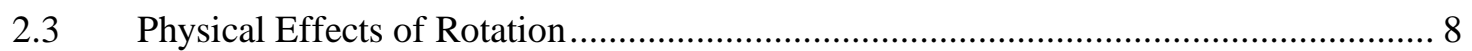

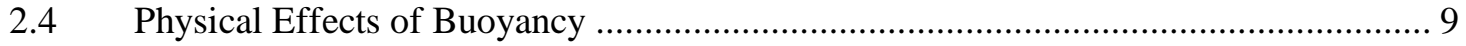

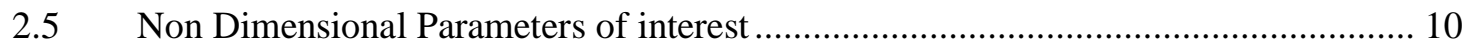

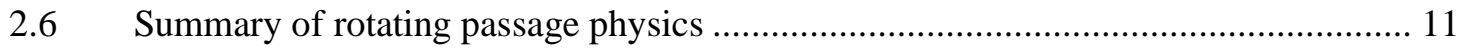

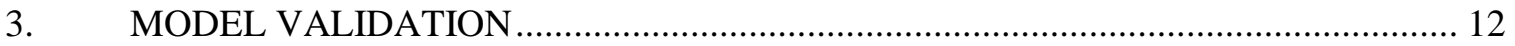

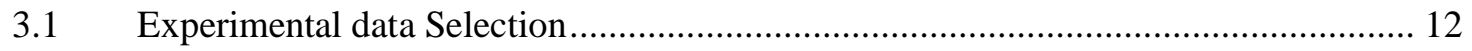

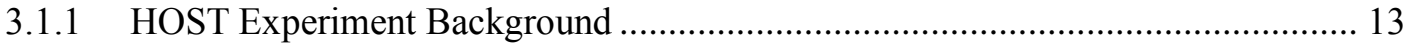

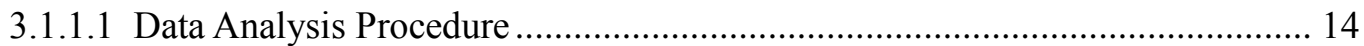

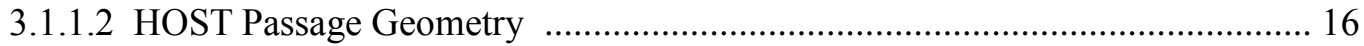

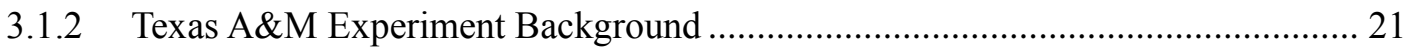

3.1.3 University of Manchester Experiment Background .......................................... 24

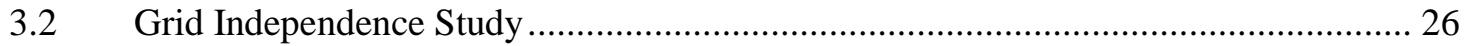

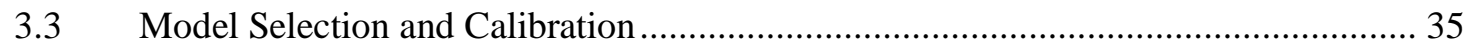

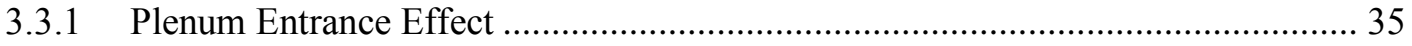

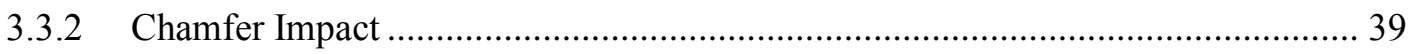

3.3.3 Simulation convergence and Physical unsteadiness ........................................ 41 


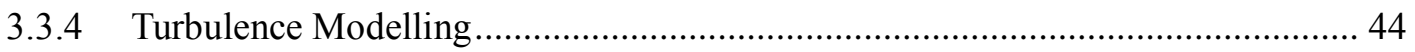

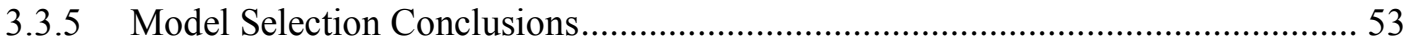

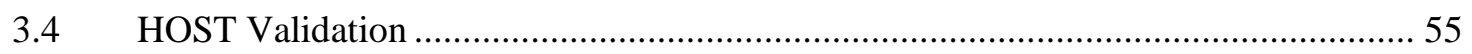

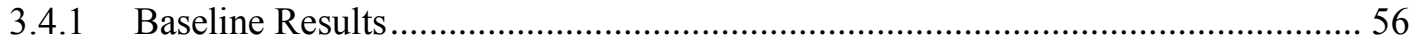

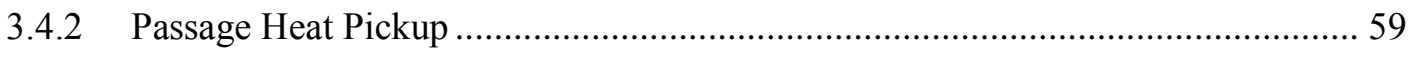

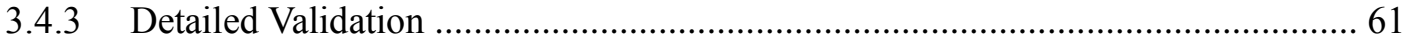

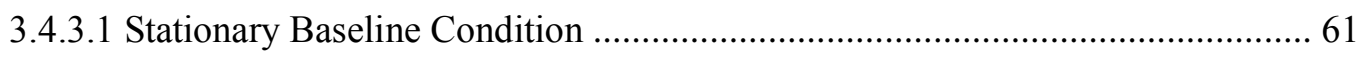

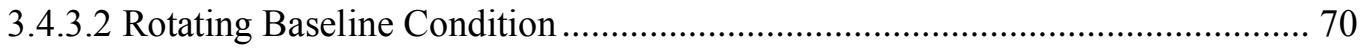

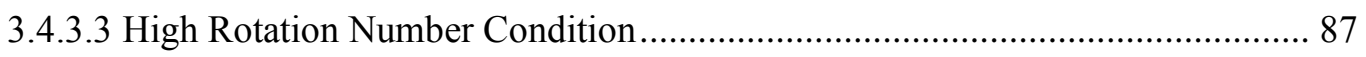

3.4.3.4 Density Ratio Variation................................................................................ 94

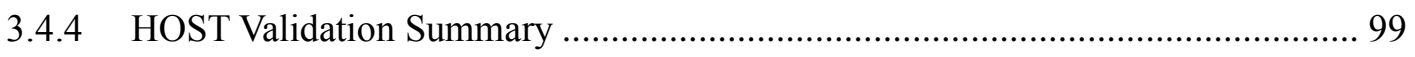

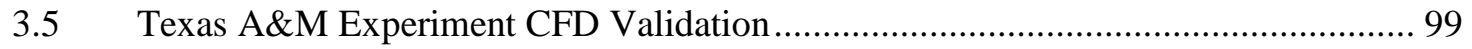

3.6 University of Manchester Experiment CFD Validation ......................................... 104

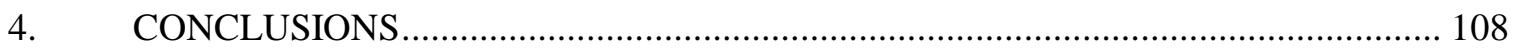

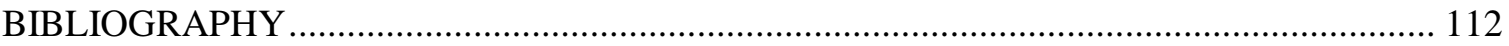

APPENDICES

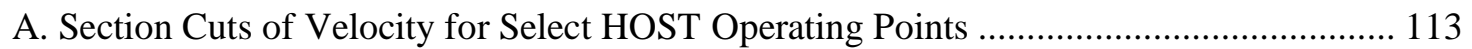




\section{LIST OF TABLES}

Table Page

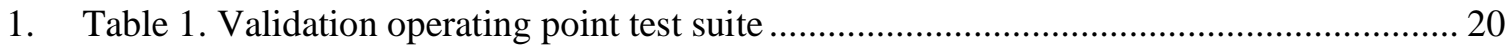

2. Table 2. Operating point used for validation against Texas A\&M data [5] ........................ 23

3. Table 3. Operating point used for validation against Iacovides experimental data .............. 25

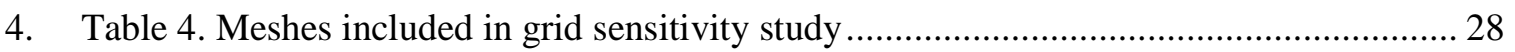

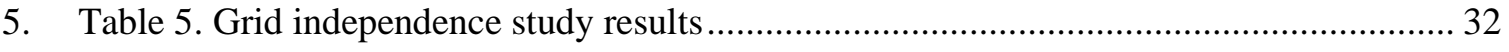

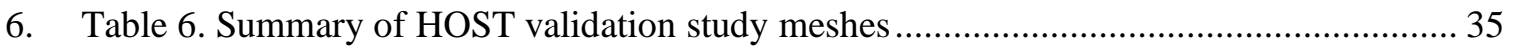

7. Table 7. Error from experiment for Turbulence model study …........................................ 51

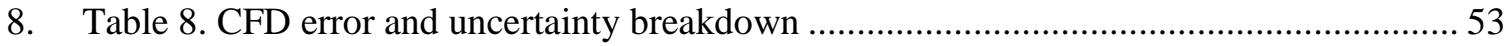

9. Table 9. Simulation setup used for Final Simulations .......................................................... 54

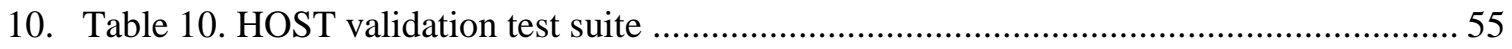

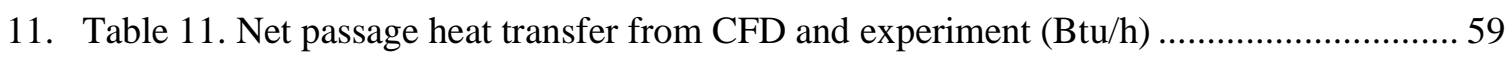

12. Table 12. Sensitivity of experiment and CFD to changes in operating condition ................. 60

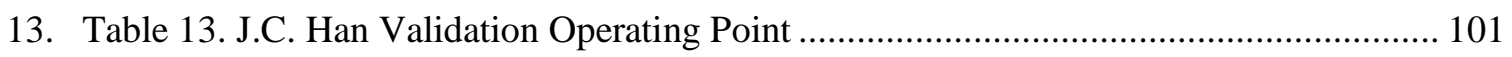




\section{LIST OF FIGURES}

Figure Page

1. Example of internal cooling geometry of a turbine blade [2] ............................................. 2

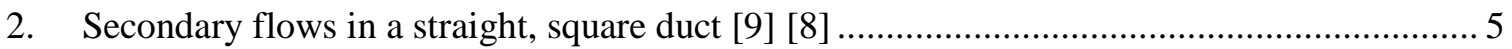

3. Steady, Non-Rotating Square Channel CFD Showing Dean Vortices; (left) view looking radially inward; (right) isometric showing projected vectors and secondary flows ............... 7

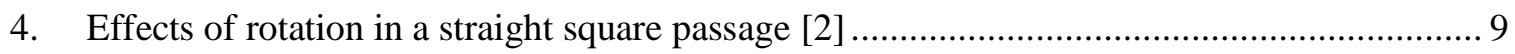

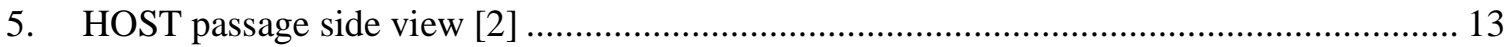

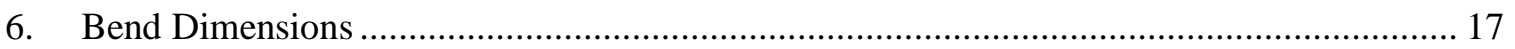

7. Host documentation of geometry [2] (black) overlaid with QuEST model (red) .................. 17

8. HOST passage geometry with various turbulator configurations ...................................... 18

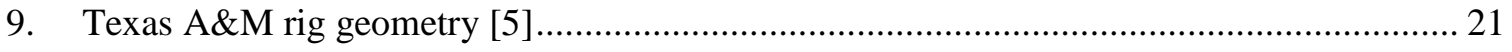

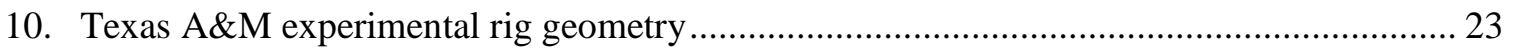

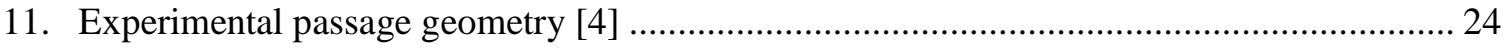

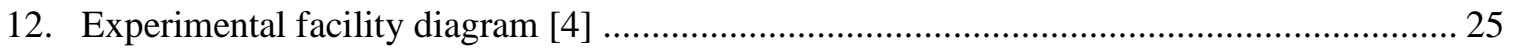

13. Leading and trailing wall Nusselt Number ratio for grid independence study ..................... 29

14. Velocity contours and mesh at mid plane with element counts specified ............................ 30

15. Comparison of secondary flow leaving the $1^{\text {st }}$ bend with different mesh types .................... 31

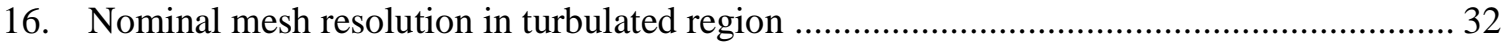

17. $\mathrm{y}+$ contours for rotating turbulated baseline simulation ................................................ 33

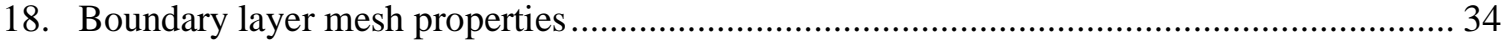

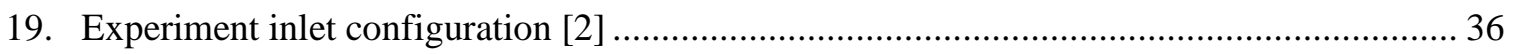

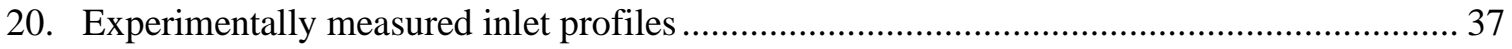

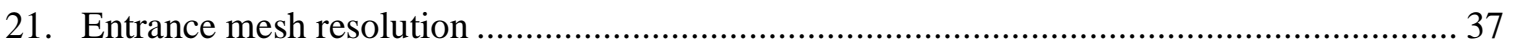

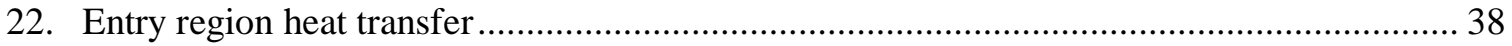

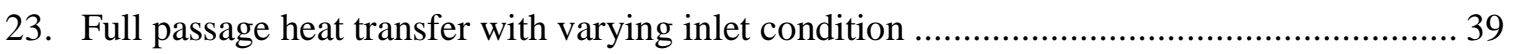

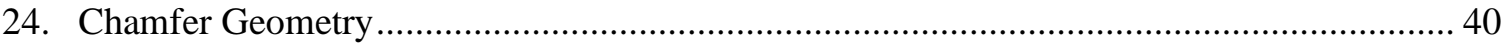

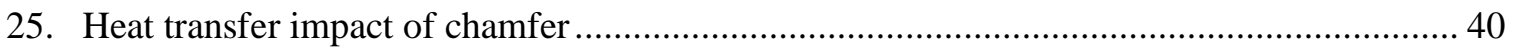

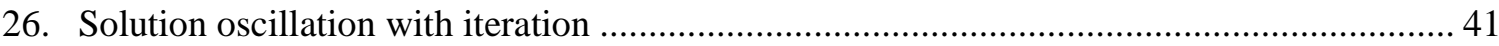

27. Heat transfer variation by station along the HOST passage ............................................. 42 
28. Steady state, pseudo averaged, and time averaged Nu\# on leading endwall at stationary baseline condition

29. Passage Nusselt Number predicted with various turbulence models

30. Wall shear contours from various turbulence models (psi)

31. Nusselt Number Ratio predicted by various turbulence models for rotating baseline operating point

32. Experiment comparison to CFD 45 degree plot

33. Side view of passage flow for non-rotating smooth HOST geometry (Case 1) .................... 56

34. Velocity visualization viewed toward leading end wall ................................................... 57

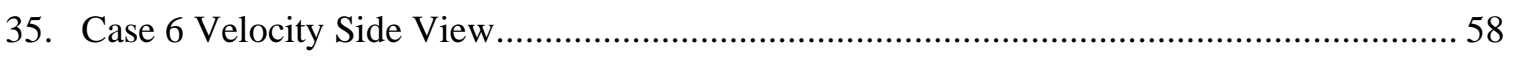

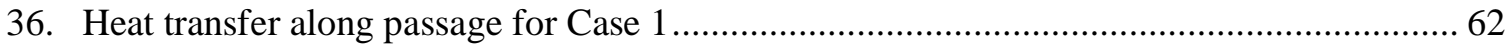

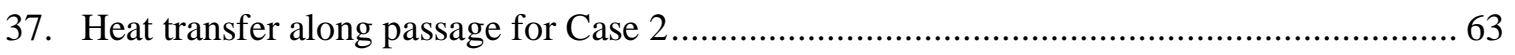

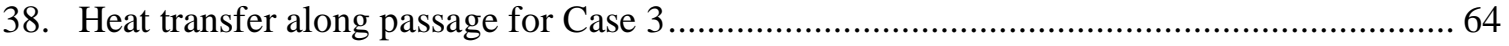

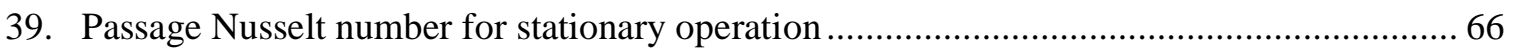

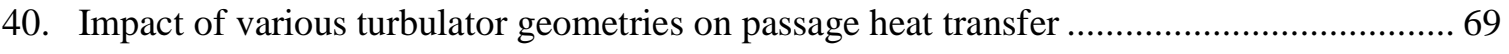

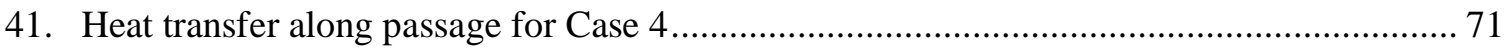

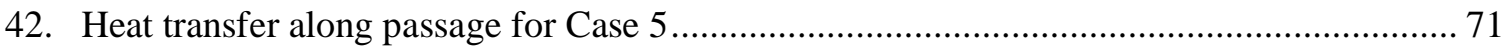

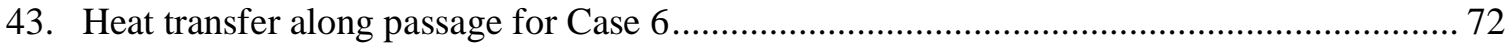

44. Nusselt number ratio for baseline rotating operating point simulations, Cases 4,5 , and $6 \ldots 74$

45. \% variation in Nusselt number associated with rotating vs. stationary baseline cases .......... 77

46. 45 degree plots representing error of baseline set of operating points ............................... 78

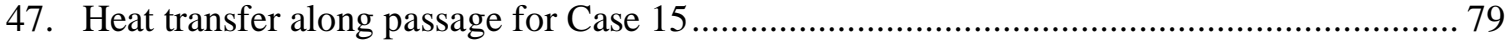

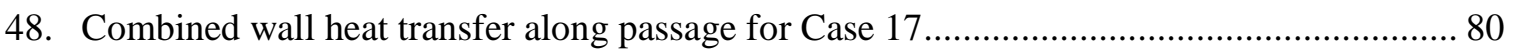

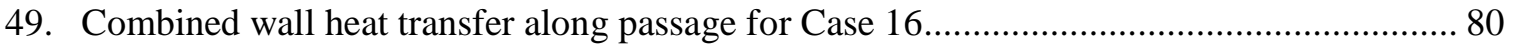

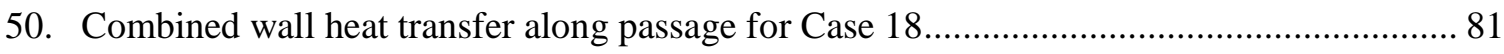

51. $\mathrm{Nu} / \mathrm{Nu}_{\infty}$ variation throughout passage due to changing Reynolds Number ......................... 83

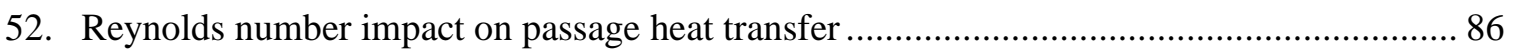

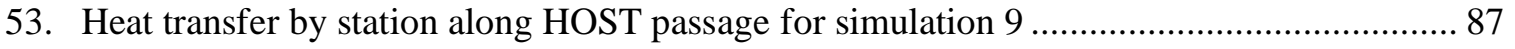

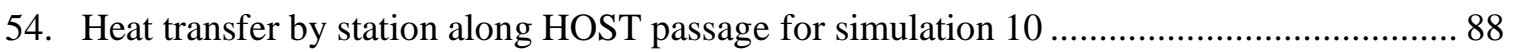

55. $\mathrm{Nu} / \mathrm{Nu}_{\infty}$ variation in passage at increased rotational speed ............................................. 90

56. \% Impact of increased rotational speed on passage heat transfer ..................................... 93 


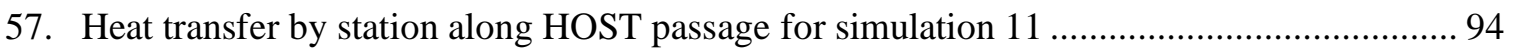

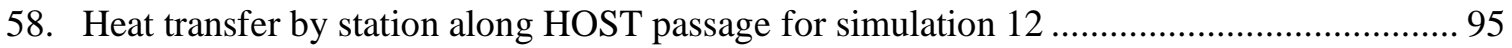

59. Heat transfer by station along HOST passage for simulation 13 ...................................... 95

60. Heat transfer by station along HOST passage for simulation 14 ...................................... 96

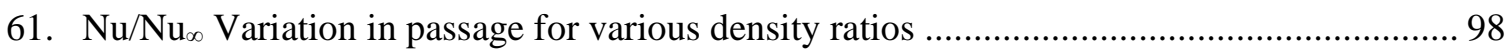

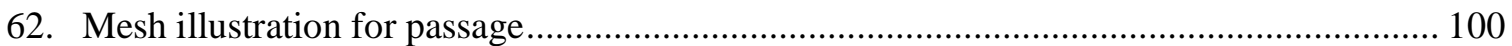

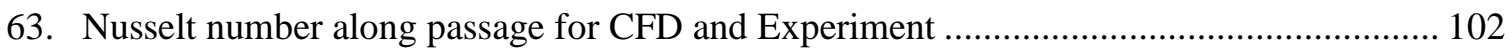

64. Difference between Leading and Trailing Nusselt number ratio due to rotation ................. 103

65. \% Deviation between experimental and CFD predicted Nusselt Number .......................... 103

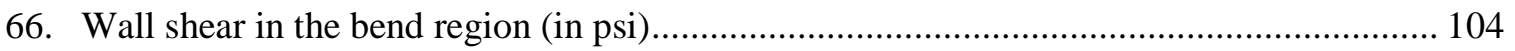

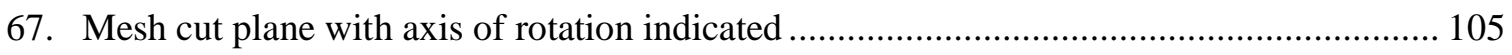

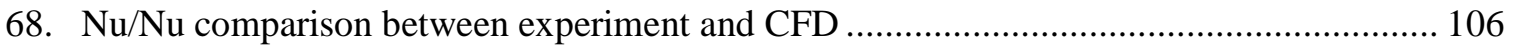

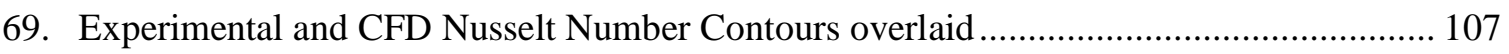




\section{DEFINITIONS AND ABBREVIATIONS}

\begin{tabular}{|c|c|}
\hline CFD & Computational Fluid Dynamics \\
\hline HOST & Hot Section Technology \\
\hline RANS & Reynolds Averaged Navier Stokes \\
\hline URANS & Unsteady Reynolds Averaged Navier Stokes \\
\hline SAS & Scale Adaptive Simulation \\
\hline SST & Shear Stress Transport \\
\hline LEVM & Linear Eddy Viscosity Model \\
\hline NLEVM & Non-Linear Eddy Viscosity Model \\
\hline RSM & Reynolds Stress Model \\
\hline $\operatorname{Re}$ & Reynolds Number \\
\hline Ro & Rotation Number \\
\hline $\operatorname{Pr}$ & Prandtl Number \\
\hline $\mathrm{Nu}$ & Nusselt Number \\
\hline Bo & Buoyancy Parameter \\
\hline h, HTC & Heat Transfer Coefficient \\
\hline Q & Heat Flow \\
\hline$k$ & thermal conductivity \\
\hline $\mathrm{k}$ & Turbulence Kinetic Energy \\
\hline$\varepsilon$ & Turbulence Dissipation \\
\hline$\omega$ & Specific Rate of Turbulence Dissipation \\
\hline$\Omega$ & Rotational speed \\
\hline A & Area \\
\hline $\mathrm{T}$ & Temperature \\
\hline $\mathrm{P}$ & Pressure \\
\hline$\rho$ & density \\
\hline$\mu$ & dynamic viscosity \\
\hline a & passage cross section dimension along axial direction \\
\hline $\mathrm{b}$ & passage cross section dimension from suction side to pressure side wall \\
\hline
\end{tabular}




\begin{tabular}{|c|c|}
\hline $\mathrm{R}$ & Radius from axis of rotation \\
\hline $\mathrm{g}_{\mathrm{c}}$ & gravitational constant \\
\hline$c_{\mathrm{p}}$ & Specific heat at constant pressure \\
\hline$C k$ & Loss coefficient \\
\hline $\mathrm{C}_{\mathrm{d}}$ & Coefficient of discharge \\
\hline $\mathrm{F} 1$ & heat transfer coefficient multiplier to account for rotation effects \\
\hline $\mathrm{F} 2$ & heat transfer coefficient multiplier to account for entrance effects \\
\hline & SUBSCRIPTS \\
\hline $\mathrm{b}$ & Bulk flow property \\
\hline $\mathrm{w}$ & Property at passage wall \\
\hline film & Film property, average of wall and bulk property \\
\hline$\infty$ & Nominal, based on a smooth straight passage \\
\hline
\end{tabular}




\section{INTRODUCTION}

This research effort is undertaken to provide practices and information which are useful in a practical sense for future cooling passage engineering efforts. Internal cooling of gas turbine rotors allows for increased firing temperatures which ultimately allow for increased power output of a given system. The understanding and prediction of turbine cooling performance translates to improvements to efficiency and capacity in power generation. A validated CFD approach for modelling the physics involved provides a valuable tool for the advancement of this understanding.

\subsection{Turbine Cooling and Serpentine Passages}

In order to achieve high efficiency, modern turbine rotors are subjected to very high temperatures. The Brayton cycle, which represents a simplified gas turbine process, indicates that the power output of a gas turbine system increases as the firing temperature at the inlet to the turbine increases. This means that it is valuable to operate gas turbines at very high temperatures.

In order to keep the metal temperature of the turbine blades within operational the range of the blade material, many measures are taken. When the primary gaspath surrounding the blade is above the allowable metal temperature, the blade temperature can be kept within acceptable limits by removing heat from the metal and insulating the blade from the hot environment. A common means for removal of heat from the turbine blade is to flow a small percentage of cool compressor bleed air through internal passages hollowed out within the blade. Often, these passages snake up and down within the blade before being exhausted into the hot gaspath, lending the name 'serpentine passages'.

An illustrative example of a serpentine passage from the NASA HOST documentation [2] is provided in Figure 1. 


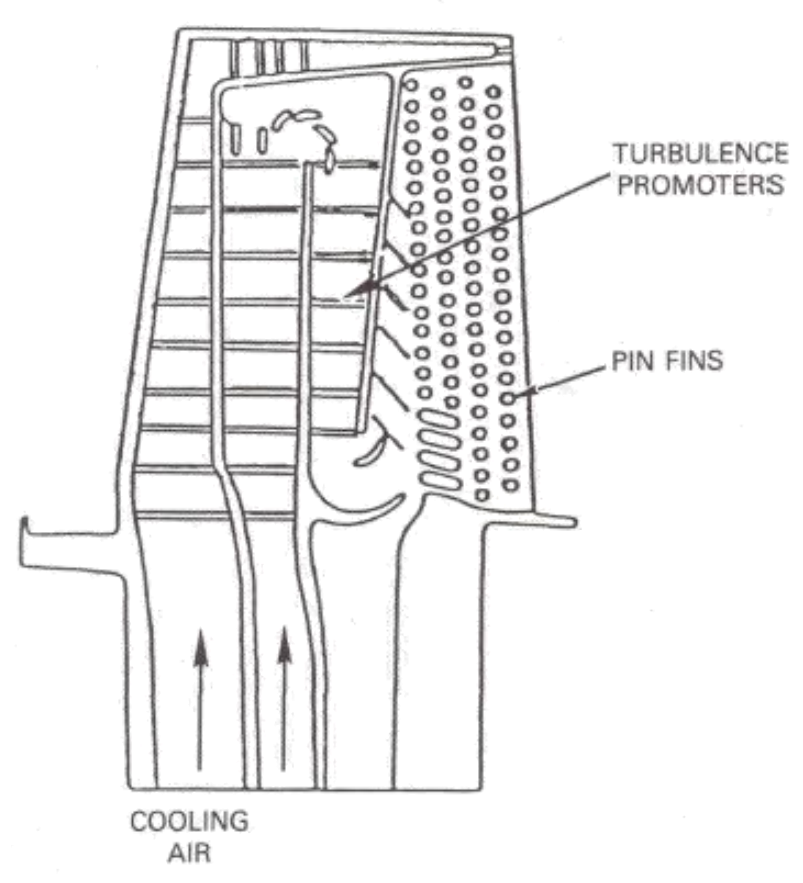

Figure 1. Example of internal cooling geometry of a turbine blade [2]

In addition to allowing increased combustion temperature and power output, use of compressor bleed air to cool turbine blades reduces the amount of inlet flow which is involved in power generation. Any flow that is bled from the compressor reduces the efficiency of the engine, so it is key to use as little compressor bleed air as possible in order to cool the turbine.

Note that other limiting factors exist which prevent arbitrarily high firing temperatures in gas turbines, such as the generation of nitrous oxide when burning hyrdrocarbons at excessive or uneven temperatures, but are not the focus of this document. This research is focused entirely on the performance and behavior of serpentine passages in blade cooling.

\subsection{Scope of Analysis}

The scope of this project and report is to evaluate the capabilities of mainstream CFD methods for heat transfer prediction inside rotating serpentine passages and develop best 
practices for internal passage modeling. Simulation is tuned and benchmarked against publically available experimental data to understand the capabilities and shortcomings of various CFD modelling approaches. This knowledge is accumulated across a wide range of operating points from various experimental programs. The majority of experimental data used comes from the NASA HOST (Hot Section Technology) program of the 1980s [2,3]. The HOST program includes experimentation across a wide range of operating parameters including Reynolds number, Rotation number, turbulator configuration, passage rotation angle, and density ratio. Additional experiments from Texas A\&M [5] and the University of Manchester [4] are used to expand model validation to additional data points.

Observations made through comparison to the described set of experimental data are used to build a modelling approach which is well understood in terms of accuracy and robustness. Model performance is considered in terms of accuracy, computational time, ease of implementation, and industry familiarity to down-select a CFD simulation approach.

The resultant data set is used to explore computational model behavior and the impact of various modelling decisions on accuracy. 


\section{PHYSICAL PHENOMENA OF INTERNAL COOLING}

This section outlines the physics associated with internal passage flow in rotating turbomachinery. Interplay between duct flow, rotation, flow turning through passage bends, and buoyancy result in a very complex flow. The section introduces simple passage flow then addresses each of the additional physical phenomena present in a rotating cooling passage.

\subsection{Flow through a rectangular passage}

Flow through a stationary square or rectangular duct has been shown by Nikuradse [7] to develop secondary flows in the passage corners. The development of these secondary flows is purely a function of anisotropic turbulence stress. The ability of CFD to predict this behavior is determined by the turbulence model selected. Most 2 equation RANS turbulence models $(\mathrm{k}-\varepsilon, \mathrm{k}-\omega)$ treat turbulence stress as an isotropic quantity, and are unable to predict the presence of corner secondary flows. It is well known that linear eddyviscosity models do not capture the anisotropic that is responsible. Figure 2 compares secondary flows predicted by various turbulence models in a square duct 

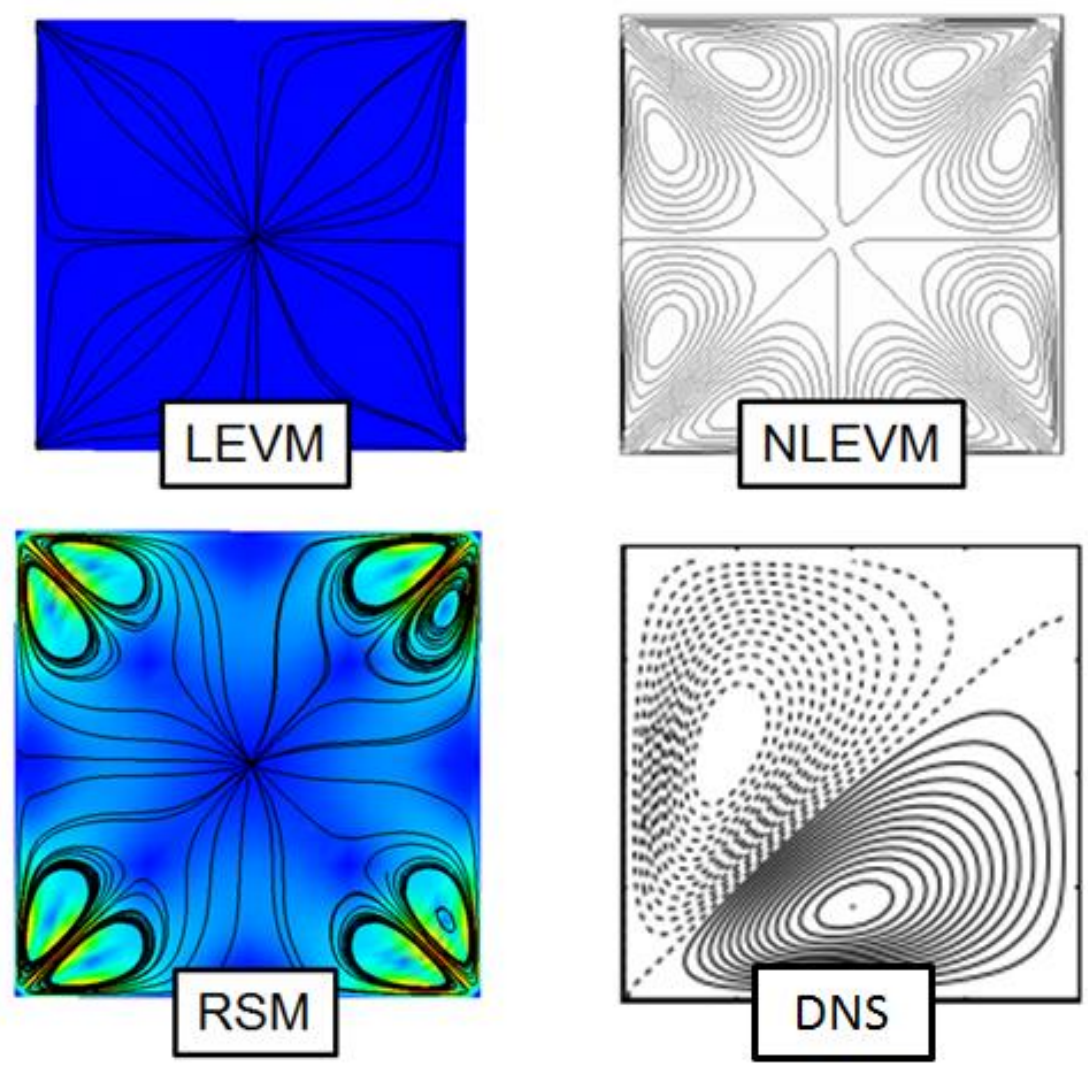

Figure 2. Secondary flows in a straight, square duct [9] [8]

LEVM refers to a linear eddy-viscosity model. NLEVM refers to a nonlinear eddyviscosity model. RSM refers to the Reynolds stress model which models turbulence anisotropically. DNS refers to direct numerical simulation of the full Navier-Stokes equations.

The level of impact these flow structures have on the heat transfer within rotating cooling passages is difficult to assess. Their presence would likely attribute to enhanced heat transfer near the corners due to higher local shear. More significant impacts could be observed when these secondary flows interplay with those due to curvature and rotation, but would be very difficult to isolate. The magnitude of bend turning, Coriolis, and buoyancy driven secondary flows is expected to dominate these corner vortices, but the interplay between them could have interesting and highly coupled effects. 


\subsection{Effects of Passage Curvature}

Research into the effect of curvature on flow goes back nearly 150 years to the study of rivers. James Thomson [1] is typically given credit with forming the third theories regarding this phenomenon. His paper describes the phenomenon such that, as water moves around a river bend, a helical secondary current is set up in the flow. While significant work is still being done to understand these geological hydrodynamic process, the early work of river scientists formed the foundation of what we now know as "Dean Vortices".

\subsubsection{Dean Vortices}

The early work of Dean [2] [3] was paramount in understand the secondary flows through curved pipes. To understand this, he started by writing the Navier-Stokes equations in toroidal form. By assuming the radius ratio ${ }^{1}$ was small, he was able to linearize the equations using the characteristic length values. While an approximation, it does capture all first-order effects. The final Dean Equations become

$$
\begin{gathered}
\frac{\partial u_{x}}{\partial x}+\frac{\partial u_{z}}{\partial z}=0 \\
K\left(\frac{D u_{x}}{D t}-u_{\phi}^{2}\right)=-K \frac{\partial p}{\partial x}+\nabla^{2} u_{x} \\
K \frac{D u_{\phi}}{D t}=1+\nabla^{2} u_{\phi} \\
K \frac{D u_{z}}{D t}=-K \frac{\partial p}{\partial z}+\nabla^{2} u_{z}
\end{gathered}
$$

Where $\mathrm{D} / \mathrm{Dt}$ is the total or substantial derivative and $\mathrm{K}$ is the Dean Number

$$
K=\frac{\rho U a}{\mu}\left(\frac{a}{b}\right)^{1 / 2}=\operatorname{Re} \sqrt{C}
$$

\footnotetext{
${ }^{1}$ Radius ratio is the ratio of pipe radius to curvature radius
} 
Where $\mathrm{C}$ is the curvature ratio. The key result from the Dean equations is that the flow is very nearly simple two-dimensional Navier-Stokes, but with an additional body force, $u_{\phi}^{2}$ acting towards the inside of the bend.
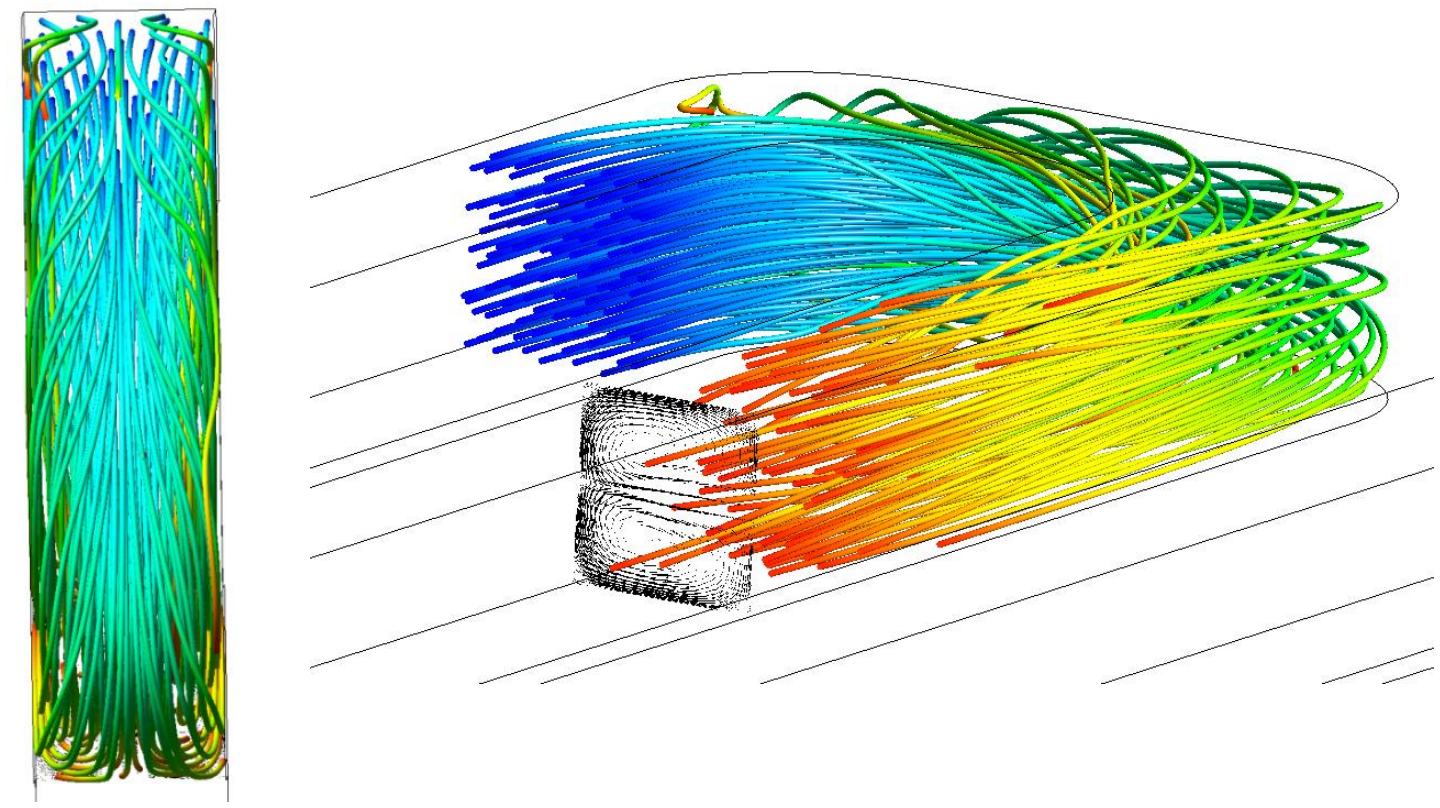

Figure 3. Steady, Non-Rotating Square Channel CFD Showing Dean Vortices; (left) view looking radially inward; (right) isometric showing projected vectors and secondary flows

\subsubsection{Görtler Vortices}

Görtler vortices present additional potential flow instability due to curvature. When the boundary layer thickness is comparable to the radius of curvature of a wall, a pressure gradient exists across the boundary layer. This pressure variation causes centrifugal instability and subsequent formation of Görtler vortices. The onset of the instability can be predicted by the non-dimensional number called the Görtler number, which is defined as 


$$
G=\frac{\rho U \theta}{\mu}\left(\frac{\theta}{R}\right)^{\frac{1}{2}}
$$

Where $\theta$ is the momentum thickness. Typically instability occurs when $\mathrm{G}>0.3$.

\subsection{Physical Effects of Rotation}

The effects of rotation are clear on a turbine cooling passage. However, correlation of these impacts is typically complex and not very robust. The issue is not only the complexity, but the difference in flow interaction each individual wall experiences. The inertial forces of rotation will create higher pressure on the pressure side wall and lower pressure on the suction side wall on outflowing passages, and higher pressure on the suction side wall for inflowing legs. This differential pressure along with the apparent Coriolis force drives flow along the sidewalls from the trailing wall to the leading wall when flow is moving away from the axis of rotation. This creates a highly destabilized boundary layer on the highpressure wall, against which the secondary flow impinges. Conversely, the leading wall has a dampened shear layer, which is much more stable. The pressure difference drives the fluid core towards the high pressure, destabilized wall. The general behavior for an outflowing straight passage is illustrated in Figure 4. 


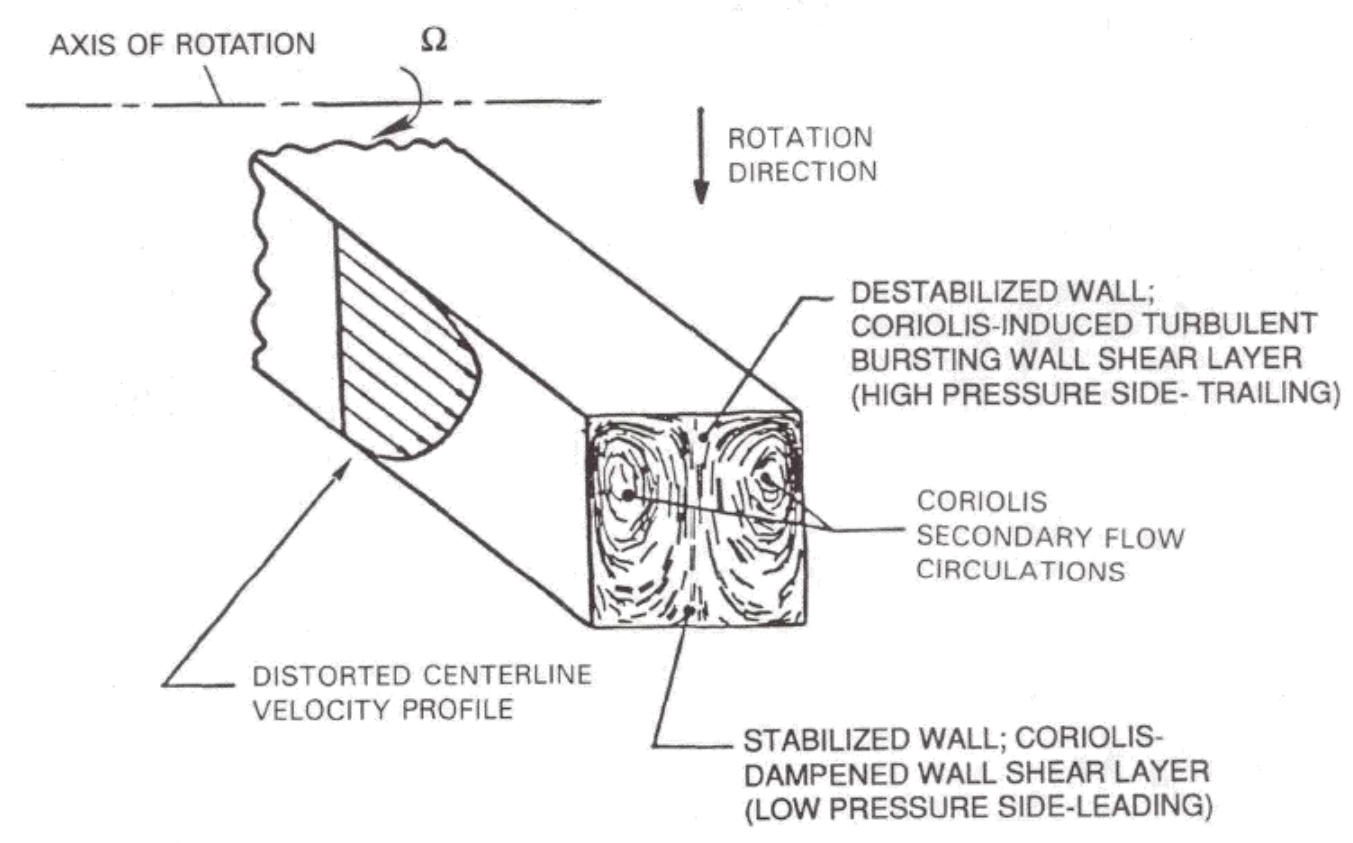

Figure 4. Effects of rotation in a straight square passage [2]

When flow is moving toward the axis of rotation, the Coriolis Effect has the opposite impact. Flow at a higher radius in a passage has a greater tangential velocity than flow at a lower radius. As air is moved from the higher radius to the lower one, it carries with it excess tangential velocity relative to the walls of the passage. This results in a motion of the bulk flow toward the leading interior wall. To compensate for this, there is a flow created along the sidewalls from the leading wall to the trailing.

It is evident why a single, unified correlation for this flow is rare, if unachievable.

\subsection{Physical Effects of Buoyancy}

Density variations create significant body forces in the presence of high rotational speed, which impact flow behavior. High rotational speeds drive denser, colder fluid toward the outer radius, pulling hotter fluid, relatively, toward the axis of rotation. For situations such as turbine cooling where the wall temperature is higher than the bulk temperature, 
buoyancy drives warmer, near-wall flow toward the inner radius. In outflowing passages this results in a buoyancy force opposite the bulk flow direction, while in inflowing legs, the buoyancy force drives near wall flow in the direction of bulk flow.

On outflowing legs, Coriolis enhances shear and heat transfer on the trailing, high pressure, wall. This creates density variation, with denser air near the leading, low pressure, wall. The existing secondary flow due to rotation interacts with the body forces due density variation, impacting the passage flow field and wall heat transfer.

When density variations across the passage cross section interact with turbulators, especially turbulators orthogonal to the flow direction, the variation in buoyancy force can drive secondary flows that would not otherwise exist. [3 p78].

Buoyancy tends to enhance pressure side wall heat transfer. On outflowing legs, this complements Coriolis behavior, enhancing the total heat transfer augmentation on the high pressure wall. On inflowing legs, this behavior acts opposite Coriolis, which enhances shear on the leading wall. Often the phenomena cancel each other out on inflowing rotating passages.

\subsection{Non Dimensional Parameters of interest}

When developing an understanding of fluid phenomena and their impacts, it is useful to specify the parameters that define the system in terms of normalized, non-dimensional parameters. Non dimensional parameters typically represent the ratio of quantities which are known to drive certain behavior. Correlations developed non-dimensionally can cover a wide range of operating points and system sizes.

Buckingham Pi theorem and the concept of dynamic similitude demonstrate the power of non-dimensional parameters. A dimensional analysis performed prior to the NASA HOST 
[2] [3] experimentation indicated that the physics of cooling flow in rotating turbomachinery can be expressed in terms of the following non-dimensional parameters.

- Density Ratio:

$$
\frac{\rho_{b}-\rho_{w}}{\rho_{b}}=\frac{T_{b}-T_{w}}{T_{b}} \text { for constant pressure ideal gas }
$$

-Rotation number:

$$
\frac{\Omega d_{h}}{V_{b}}
$$

- Buoyancy Parameter:

$$
\left[\frac{\rho_{b}-\rho_{w}}{\rho_{b}}\right]\left[\frac{\Omega d_{h}}{V_{b}}\right]\left[\frac{\Omega R}{V_{b}}\right]=\text { Density Ratio } * \text { Ro\# } *\left[\frac{\Omega R}{V_{b}}\right]
$$

- Reynolds Number: $\quad \frac{\rho_{b} V_{b} d_{h}}{\mu_{b}}$

Notice that these parameters quantify the magnitude and impact of the physical phenomena described in this section, relative to each other.

\subsection{Summary of rotating passage physics}

Many of the physical phenomena described in this section are difficult to predict and understand on their own. In rotating turbomachinery, these impacts and others interact in ways that are extremely difficult to predict analytically. Additionally, it is very difficult to isolate the effects from one another using experimental data, which only provides information for which instrumentation is in place to extract. Numerical models such as CFD present a unique opportunity to capture each of these phenomena and their interaction without making assumptions aside from those inherent to the computational model. The behavior of the fluid, as governed by the computational model used, can be interrogated freely for any and all features of the flow modelled, at any location or region within the simulation domain. A CFD model validated against experiment which captures these physical interactions is invaluable in the creation of updated correlations. Increased understanding of the flowfield and the ability to simulate flow behavior outside the range of experimental data are key objectives for the CFD model which is to be developed. 


\section{MODEL VALIDATION}

The first phase of the present effort consists of developing a CFD model of academic internal cooling applications and validating against experimental data. Primary characteristics of the model which need to be validated are mesh type, mesh resolution, Turbulence model, and treatment of unsteadiness in model.

\subsection{Experimental data Selection}

The NASA HOST (Hot Section Technology) [1,2] test suite represents the most comprehensive publically available data set for rotating internal passages. The project was undertaken in the late 1980 's to investigate "heat transfer characteristics of rotating multipass passages" of turbine blades from that period. Sensitivity of passage heat transfer to Reynolds Number, Rotation Number, Density Ratio, and Turbulator configuration were studied experimentally. The extent and availability of the HOST experimental data has led to its use in the generation of correlations and general understanding of serpentine cooling passage physics throughout the gas turbine industry.

One issue encountered when validating CFD against the HOST data is that the bend geometry is not explicitly described in available literature. Diagrams and photographs are digitized to extract the geometry used in the CFD model described herein.

Additional experimental data is included in the validation to improve confidence and filter out any uncertainties exclusive to the HOST experiment, including variations between the bend geometry used in the current model and the experimental bend geometry.

Je-Chin Han of Texas A\&M [5] has published several papers regarding heat transfer within serpentine passages. One set of J.C. Han experimental data was included in the validation phase of the current effort due to complete documentation of test article geometry, and more modern 
instrumentation. Experimental data for wall heat transfer is collected at a higher resolution than in the HOST program, and the geometry is fully described in the documentation.

Hector Iacovides of the University of Manchester has also conducted experimental research into heat transfer within rotating passages. Experimental results [4] are included in this validation because the experiment includes liquid crystal imaging, providing experimental contours of heat transfer, which can be validated against in 2-D. Some uncertainties in the test setup and postprocessing have arisen which will be discussed later.

\subsubsection{HOST Experiment Background}

The HOST test article consists of a duct with 4 legs and 3 bends. The side view of the passage is illustrated in Figure 5.

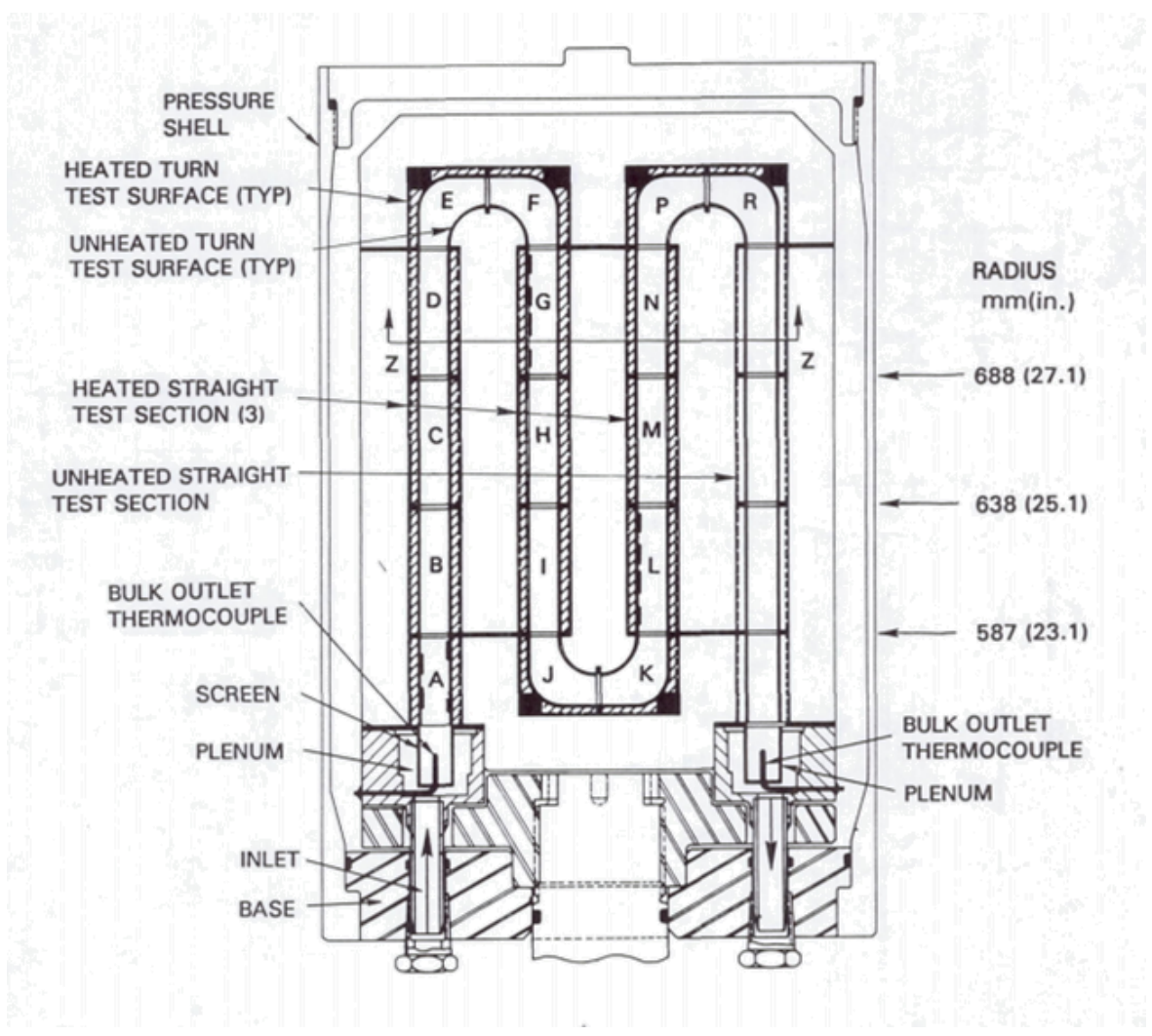

Figure 5. HOST passage side view [2] 
The geometry is made up of a square cross section, 4 pass duct. The walls of the duct are split into plates which are labelled by letter in the image above. Heat transfer data is extracted from the experiment by performing an energy balance to solve for the heat transferred through each plate. Bulk temperature and material properties are calculated as an average for each plate's streamwise location to account for heat up. Error associated with the energy balance for plate heat flux cited by the HOST documentation [2] is $\pm 2 \%$ for the $1^{\text {st }}$ leg, $\pm 4 \%$ for the $2^{\text {nd }}$ leg, and $\pm 7 \%$ for the final heated leg. This error is primarily due to compounding error in the calculation of bulk temperature along the passage.

\subsubsection{Data Analysis Procedure}

The HOST published data is presented in terms of Nusselt number. The integrated heat flux through each plate is converted to a heat transfer coefficient by the following calculation.

$$
h=\frac{Q}{A *\left(T_{w}-T_{\text {Film }}\right)}
$$

Where $T_{\text {film }}$ is defined as the average of the bulk fluid temperature and wall temperature.

$$
T_{f i l m}=\frac{T_{w}+T_{b}}{2}
$$

The HTC can then be normalized as a Nusselt number based on the hydraulic diameter and fluid thermal conductivity.

$$
N u=\frac{h * D_{h}}{k}
$$

In many cases, the Nusselt number is further normalized, as $\mathrm{Nu} / \mathrm{Nu}_{\text {inf. }}$. Where $\mathrm{Nu}_{\text {inf }}$ represents the expected Nusselt number for a corresponding fully developed turbulent duct. $\mathrm{Nu}_{\text {inf }}$ is calculated based on the Dittus Boelter equation. 


$$
N u_{\text {inf }}=0.023 \operatorname{Re}^{0.8} \operatorname{Pr}^{0.4}
$$

The HOST data uses the Colburn equation instead of Dittus-Boelter, assuming a constant Prandtl number equal to 0.72 .

$$
N u_{\text {inf }}=0.0176 R e^{0.8}
$$

This research uses the HOST calculation for $\mathrm{Nu}_{\text {inf }}$ when comparing to HOST data, and Dittus Boelter when comparing to other data sets.

The bulk temperature used in calculation of the HTC is an important factor. The NASA HOST program calculated the bulk fluid temperature at each station along the serpentine passage by integrating heat addition through all plates and using fluid $\mathrm{Cp}$ to calculate a temperature difference from the previous station.

CFD allows the direct postprocessing of local bulk temperature, rather than using an estimation based on heat addition. When validating the CFD model against HOST data, the HOST methodology for updating bulk temperature was applied to the CFD results. Bulk temperature is updated at each station along the HOST test passage according to.

$$
T_{i}=T_{i-1}+\frac{Q_{i-1}}{c_{p_{i-1}}}
$$

Where $i$ is an index which represents the current region, associated with a set of heated plates in the experimental rig. The index, $i$ increases in the streamwise direction. $\mathrm{Q}_{\mathrm{i}}$ represents the integrated heat flux through all heated plates at a given indexed region.

Inlet temperature is prescribed, so the temperature throughout the passage is calculated by marching the above equation along the flow direction. Marching the fluid temperature represents a low-order, one sided discretization of the differential equation for conservation of thermal 
energy. In the presence of sharp gradients, this approach could lead to appreciable numerical error. For that reason, the result has been compared against arithmetic mean, and log-mean temperature differences to ensure minimal error, well below $1 \%$.

Polynomial representation of air properties in terms of temperature is used to update $\mathrm{c}_{\mathrm{p}}, \mu$, and $k$ at each station. Pressure data is interrogated from the CFD results and used in combination with bulk temperature to calculate local density based on the ideal gas equation of state.

$$
P=\rho R T
$$

Note that Re\# and Pr\# vary from station to station based on changes in material properties. Density, for example, is directly coupled to flow velocity through the continuity equation in a closed passage at steady state.

$$
\rho V A=\text { constant }
$$

The Nusselt number ratio, $\mathrm{Nu} / \mathrm{Nu}_{\text {inf }}$ can be thought of as the ratio of heat transfer observed in the experiment to the heat transfer that would be observed in a smooth straight tube. For example, if a straight turbulated duct exhibits a $\mathrm{Nu} / \mathrm{Nu}_{\text {inf }}$ of 2, this means that the presence of turbulators has doubled the heat transfer through the duct walls relative to a smooth passage. The data available from the HOST documentation is primarily in terms of Nusselt number ratio for each of the lettered stations in Figure 5 for each of the 4 walls of the passage, independently.

\subsubsection{HOST Passage Geometry}

QuEST was unable to find any explicit documentation for some aspects of the HOST test article geometry. Specifically, the bend inner radius and tip radius within the bend were not documented. Digitizing images of the test article and referring to other CFD studies that validated against HOST (Sleiti), led to the tip geometry shown in Figure 6. 


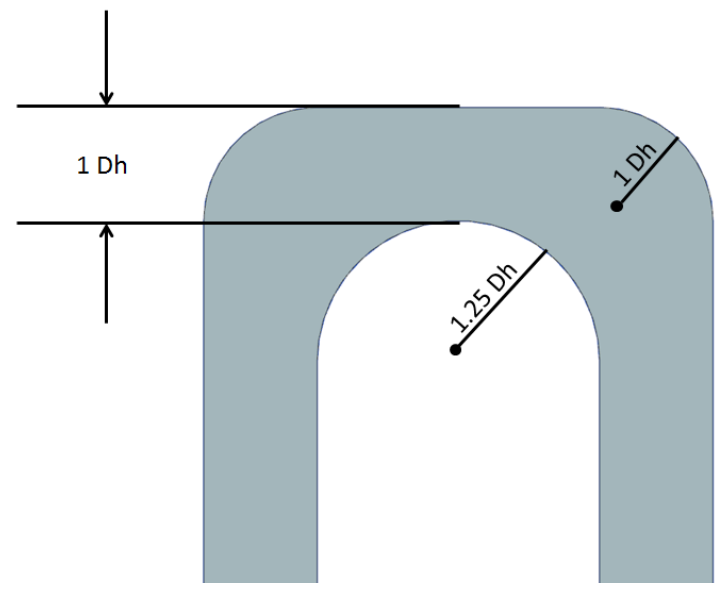

Figure 6. Bend Dimensions

The inner radius of the bends is $1.25 \mathrm{Dh}$. The outer radius of the bends at the corners is $1 \mathrm{Dh}$. The minimum passage height, halfway through the bend is equal to the hydraulic diameter of the straight sections. The comparison of this geometry to an image from HOST documentation is shown in Figure 7.

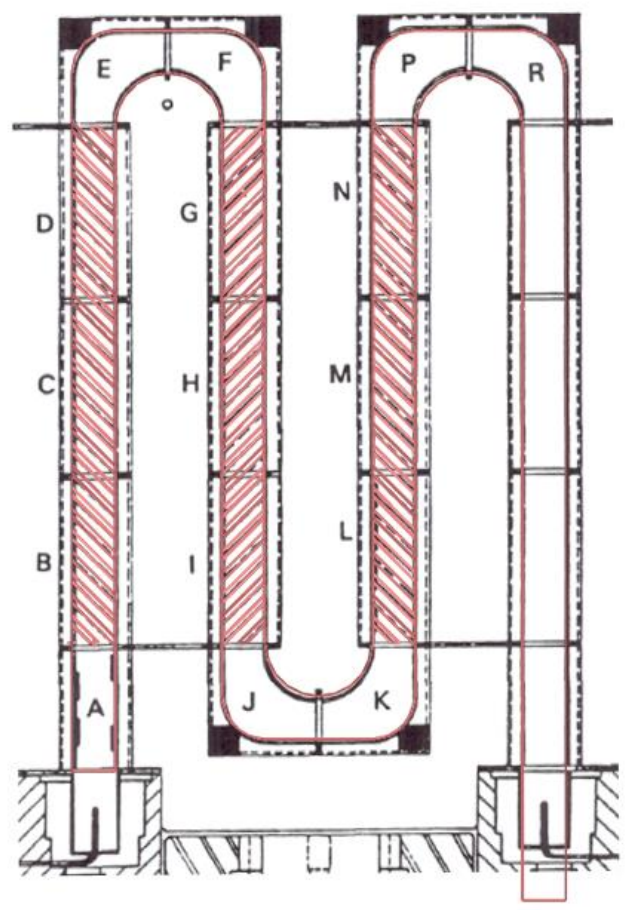

Figure 7. Host documentation of geometry [2] (black) overlaid with QuEST model (red) 
The geometry generated for this analysis is overlaid in red over an illustration from HOST Volume 2 [3].

3D CAD of the HOST passage was generated and is illustrated in Figure 8.

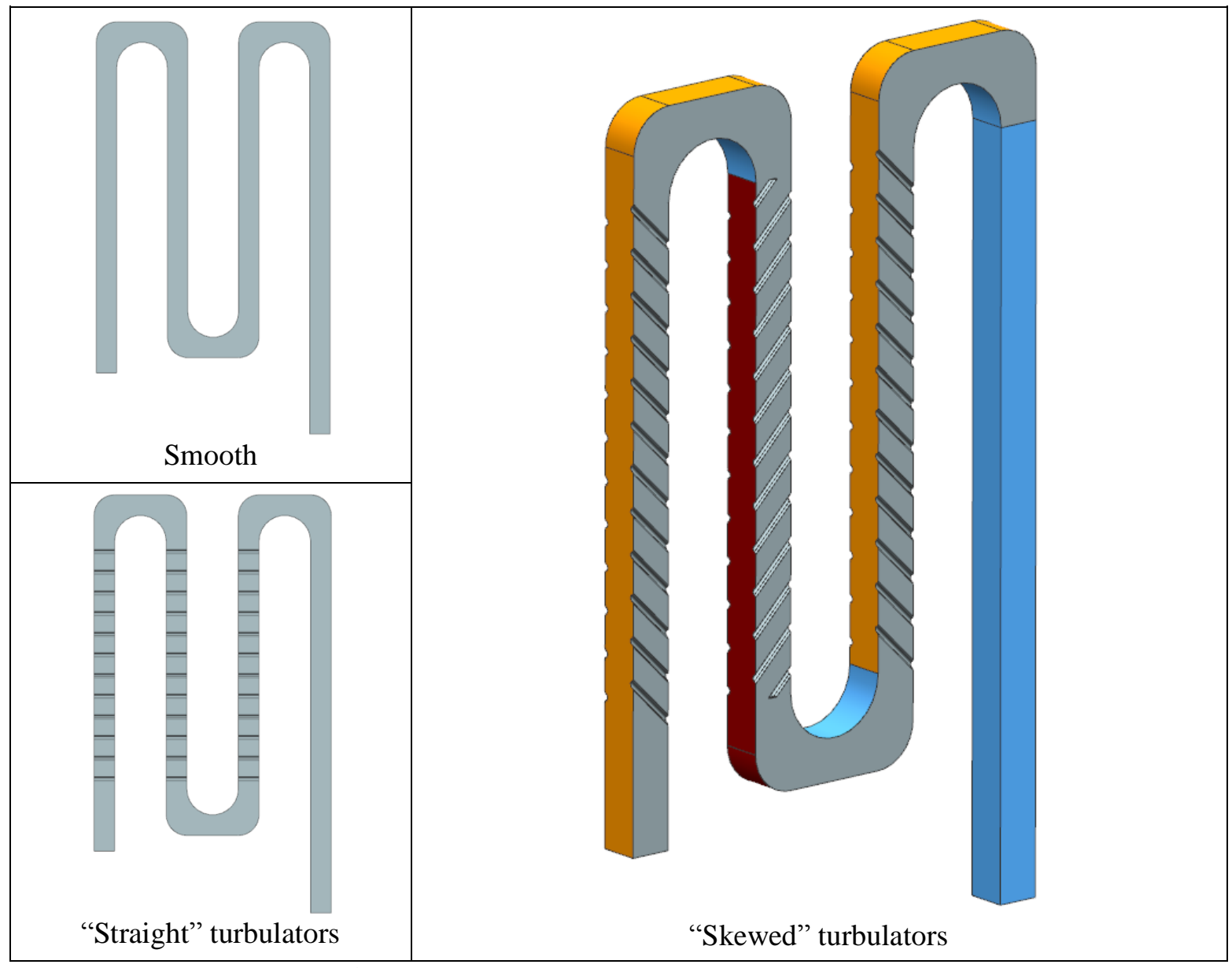

Figure 8. HOST passage geometry with various turbulator configurations

On the right in figure 6 , the non turbulated walls are color coded by region. The nomenclature used in this report refers to the orange surfaces shown as the "outer" sidewall. The red surfaces are referred to as the "inner" sidewall. Any blue surface is unheated and was treated as isothermal in the CFD and HOST experiment, including the inner radius of each bend. The turbulated walls are referred to as the "leading" and "trailing" endwalls. When the passage is rotating, the leading endwall is the wall visible in Figure 8, which leads the rotation, corresponding to the suction side of a turbine blade cooling passage. 
A range of experimental test points were selected from the HOST program results to validate the CFD model against. The set of operating points included in the validation is summarized in

\begin{tabular}{|c|c|c|c|c|c|c|c|}
\hline Case & $\begin{array}{c}\mathbf{R e} \\
\text { Number }\end{array}$ & $\begin{array}{c}\text { Ro } \\
\text { Number }\end{array}$ & $\begin{array}{c}\text { Radius } \\
\text { Ratio }(\mathbf{R} / \mathbf{d})\end{array}$ & $\begin{array}{c}\text { Rotation } \\
\text { Angle }\end{array}$ & $\begin{array}{c}\text { Inlet } \\
\text { Density } \\
\text { Ratio }\end{array}$ & Turbulators & Purpose \\
\hline 1 & 25,000 & 0 & 49 & 0 & 0.13 & None & \multirow{3}{*}{$\begin{array}{l}\text { Baseline } \\
\text { Stationary }\end{array}$} \\
\hline 2 & 25,000 & 0 & 49 & 0 & 0.13 & Straight & \\
\hline 3 & 25,000 & 0 & 49 & 0 & 0.13 & $45 \mathrm{deg}$ & \\
\hline 4 & 25,000 & 0.24 & 49 & 0 & 0.13 & None & \multirow{3}{*}{$\begin{array}{l}\text { Baseline } \\
\text { Rotating }\end{array}$} \\
\hline 5 & 25,000 & 0.24 & 49 & 0 & 0.13 & Straight & \\
\hline 6 & 25,000 & 0.24 & 49 & 0 & 0.13 & $45 \mathrm{deg}$ & \\
\hline 7 & 25,000 & 0.34 & 49 & 0 & 0.13 & None & \multirow{2}{*}{$\begin{array}{l}\text { Effect of } \\
\text { Rotation } \\
\text { Number }\end{array}$} \\
\hline 8 & 25,000 & 0.34 & 49 & 0 & 0.13 & $45 \mathrm{deg}$ & \\
\hline 9 & 25,000 & 0.24 & 49 & 0 & 0.07 & None & \multirow{4}{*}{$\begin{array}{c}\text { Effect of } \\
\text { Density } \\
\text { Ratio }\end{array}$} \\
\hline 10 & 25,000 & 0.24 & 49 & 0 & 0.23 & None & \\
\hline 11 & 25,000 & 0.24 & 49 & 0 & 0.07 & $45 \mathrm{deg}$ & \\
\hline 12 & 25,000 & 0.24 & 49 & 0 & 0.23 & $45 \mathrm{deg}$ & \\
\hline 13 & 12,500 & 0.12 & 49 & 0 & 0.13 & None & \multirow{4}{*}{$\begin{array}{c}\text { Effect of } \\
\text { Reynolds } \\
\text { Number }\end{array}$} \\
\hline 14 & 50,000 & 0.12 & 49 & 0 & 0.13 & None & \\
\hline 15 & 12,500 & 0.12 & 49 & 0 & 0.13 & $45 \mathrm{deg}$ & \\
\hline 16 & 75,000 & 0.12 & 49 & 0 & 0.13 & $45 \mathrm{deg}$ & \\
\hline
\end{tabular}


Table 1. Validation operating point test suite

\begin{tabular}{|c|c|c|c|c|c|c|c|}
\hline Case & $\begin{array}{c}\mathbf{R e} \\
\text { Number }\end{array}$ & $\begin{array}{c}\text { Ro } \\
\text { Number }\end{array}$ & $\begin{array}{c}\text { Radius } \\
\text { Ratio }(\mathbf{R} / \mathbf{d})\end{array}$ & $\begin{array}{c}\text { Rotation } \\
\text { Angle }\end{array}$ & $\begin{array}{c}\text { Inlet } \\
\text { Density } \\
\text { Ratio }\end{array}$ & Turbulators & Purpose \\
\hline 1 & 25,000 & 0 & 49 & 0 & 0.13 & None & \multirow{3}{*}{$\begin{array}{l}\text { Baseline } \\
\text { Stationary }\end{array}$} \\
\hline 2 & 25,000 & 0 & 49 & 0 & 0.13 & Straight & \\
\hline 3 & 25,000 & 0 & 49 & 0 & 0.13 & $45 \mathrm{deg}$ & \\
\hline 4 & 25,000 & 0.24 & 49 & 0 & 0.13 & None & \multirow{3}{*}{$\begin{array}{l}\text { Baseline } \\
\text { Rotating }\end{array}$} \\
\hline 5 & 25,000 & 0.24 & 49 & 0 & 0.13 & Straight & \\
\hline 6 & 25,000 & 0.24 & 49 & 0 & 0.13 & $45 \mathrm{deg}$ & \\
\hline 7 & 25,000 & 0.34 & 49 & 0 & 0.13 & None & \multirow{2}{*}{$\begin{array}{l}\text { Effect of } \\
\text { Rotation } \\
\text { Number }\end{array}$} \\
\hline 8 & 25,000 & 0.34 & 49 & 0 & 0.13 & $45 \mathrm{deg}$ & \\
\hline 9 & 25,000 & 0.24 & 49 & 0 & 0.07 & None & \multirow{4}{*}{$\begin{array}{c}\text { Effect of } \\
\text { Density } \\
\text { Ratio }\end{array}$} \\
\hline 10 & 25,000 & 0.24 & 49 & 0 & 0.23 & None & \\
\hline 11 & 25,000 & 0.24 & 49 & 0 & 0.07 & $45 \mathrm{deg}$ & \\
\hline 12 & 25,000 & 0.24 & 49 & 0 & 0.23 & $45 \mathrm{deg}$ & \\
\hline 13 & 12,500 & 0.12 & 49 & 0 & 0.13 & None & \multirow{4}{*}{$\begin{array}{c}\text { Effect of } \\
\text { Reynolds } \\
\text { Number }\end{array}$} \\
\hline 14 & 50,000 & 0.12 & 49 & 0 & 0.13 & None & \\
\hline 15 & 12,500 & 0.12 & 49 & 0 & 0.13 & $45 \mathrm{deg}$ & \\
\hline 16 & 75,000 & 0.12 & 49 & 0 & 0.13 & $45 \mathrm{deg}$ & \\
\hline
\end{tabular}

As indicated, the baseline operating condition is at a Reynolds number of 25,000 and an inlet density ratio of 0.13 . The Rotation number is 0.24 in the rotating baseline case. Other cases vary parameters away from the baseline case to evaluate the impact of changes in those parameters and assess the capability of the CFD model to capture those impacts. 
Validation against HOST program data comprises the bulk of the current validation effort. A wide range of available data at varying operating points allows for a comprehensive look at modelling capability.

\subsubsection{Texas A\&M Experiment Background}

Experimental heat transfer measurements in a u-bend conducted by Je-Chin Han of Texas A\&M are also included in the validation of the current CFD model. Experiments documented include many aspect ratios and operating points of a 2 pass rotating duct. Figure 9 illustrates the rig geometry selected for validation herein.

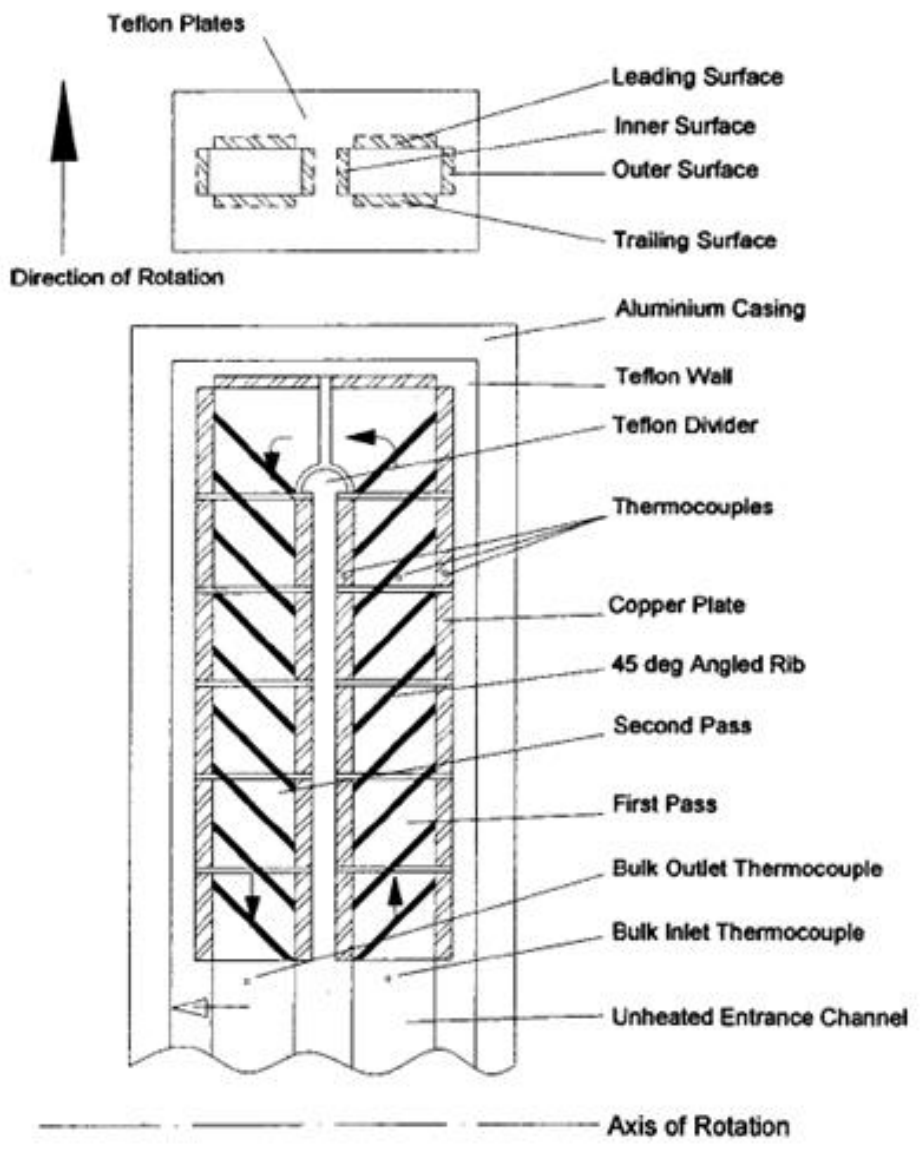

Figure 9. Texas A\&M rig geometry [5] 
Heated plates indicated with cross-hatching surround the test section similarly to the HOST experiment. The interior bend radius is unheated. The aspect ratio of the duct is 2 , with the suction and pressure side wall larger than the outer and inner sidewalls. Turbulators are not staggered, meaning that the pressure side and suction side turbulators extend into the flow passage at the same radial location, resulting in a net choking down of the passage dimension $b$ equivalent to $2 \mathrm{e}$.

The turbulators are feature sharp corners, unlike those considered for the HOST experiment which had rounded edges. Heat flux is measured through heated plates on the leading and trailing walls. These measurements for the leading and trailing endwall can be compared to CFD results. The CAD geometry created for the analysis is illustrated in Figure 10. 


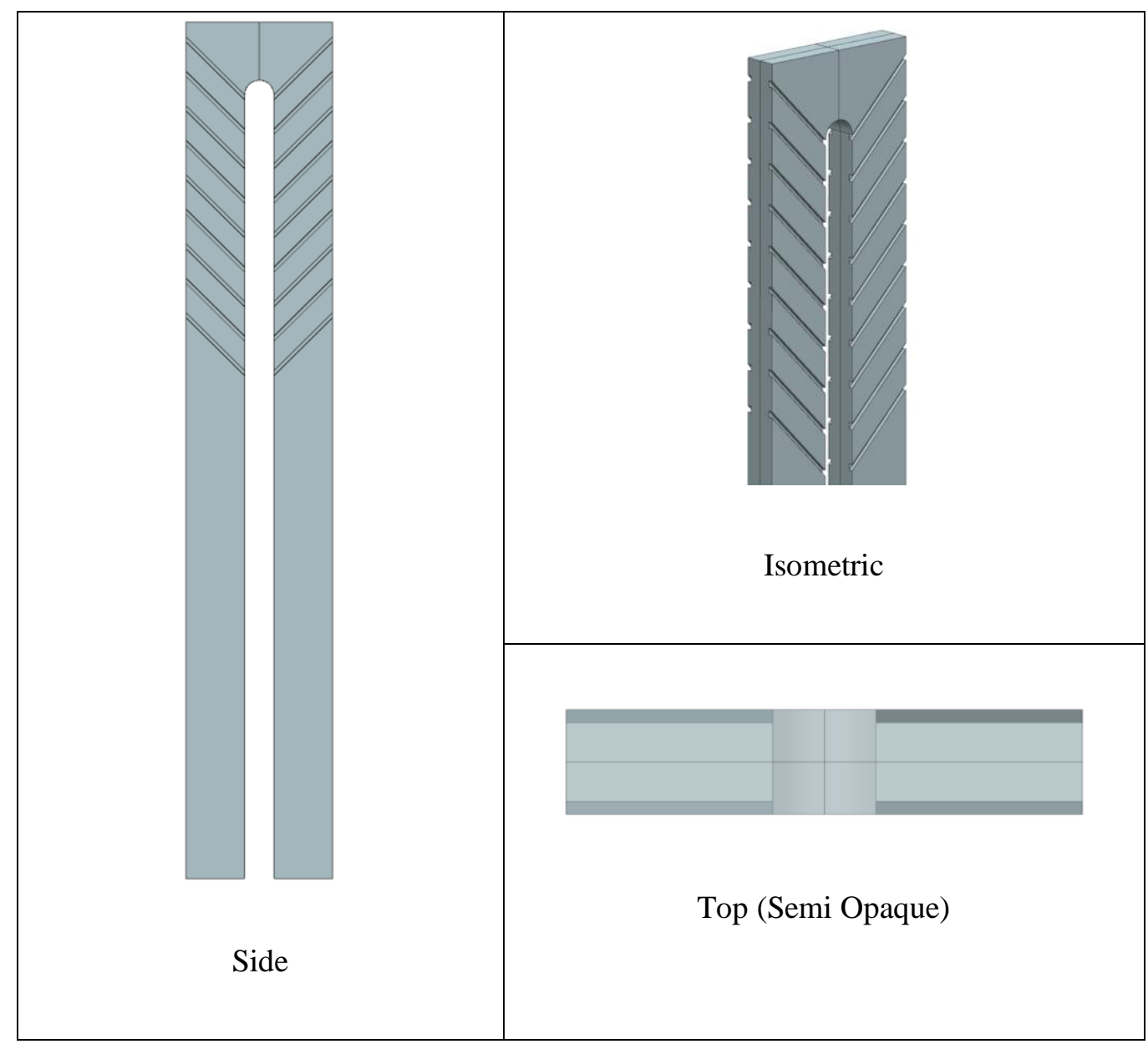

Figure 10. Texas A\&M experimental rig geometry

The operating point and geometric parameters chosen for comparison to CFD is outlined in Table 2.

Table 2. Operating point used for validation against Texas A\&M data [5]

\begin{tabular}{|c|c|}
\hline Parameter & Value \\
\hline Ro \# & 0.21 \\
\hline Reynolds \# & 25,000 \\
\hline Density Ratio $\frac{\Delta \rho}{\rho}$ & 0.115 \\
\hline Rib height to hydraulic diameter $\left(\mathrm{e} / \mathrm{D}_{\mathrm{h}}\right)$ & 0.094 \\
\hline Rib pitch-to-height $(\mathrm{P} / \mathrm{e})$ & 10 \\
\hline
\end{tabular}


In this case rotation number is non-dimensionalized by $b$, the distance from the suction side wall to the pressure side wall. The J.C. Han experiment is included in this validation because the experimental data is more recent, the geometry is explicitly fully defined, and the number of plates, and therefore resolution of test data, is increased compared to the HOST data.

\subsubsection{University of Manchester Experiment Background}

The experiment conducted by Iacovides of University of Manchester [4] consists of a 2 pass duct which is rotated on a turntable. The inflowing leg of the duct has a square cross section. The downstream leg expands to a 2:1 aspect ratio. The passage geometry is outlined in Figure 11.

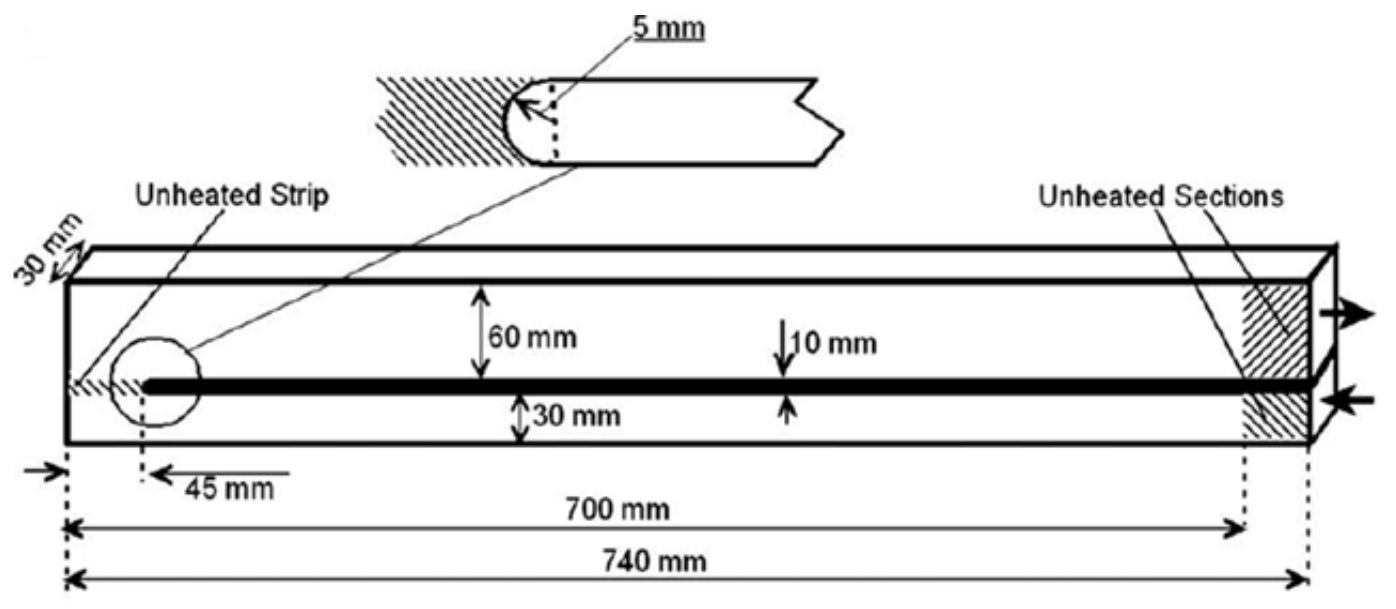

Figure 11. Experimental passage geometry [4]

The reason for inclusion of this experiment in the model validation is that liquid crystals are used to generate experimental contours of Nusselt Number on the suction and pressure side walls. This 2-D representation of experimental data allows for a more qualitative validation of the CFD model physics. Stationary experiments were conducted at various Reynolds numbers with air and water as the working fluid. Rotating experiments were then conducted with water as the working fluid. The passage was mounted to a turntable within a chamber. For rotating experiments, the chamber was filled with water. The flowpath and experimental setup are illustrated in Figure 12. 


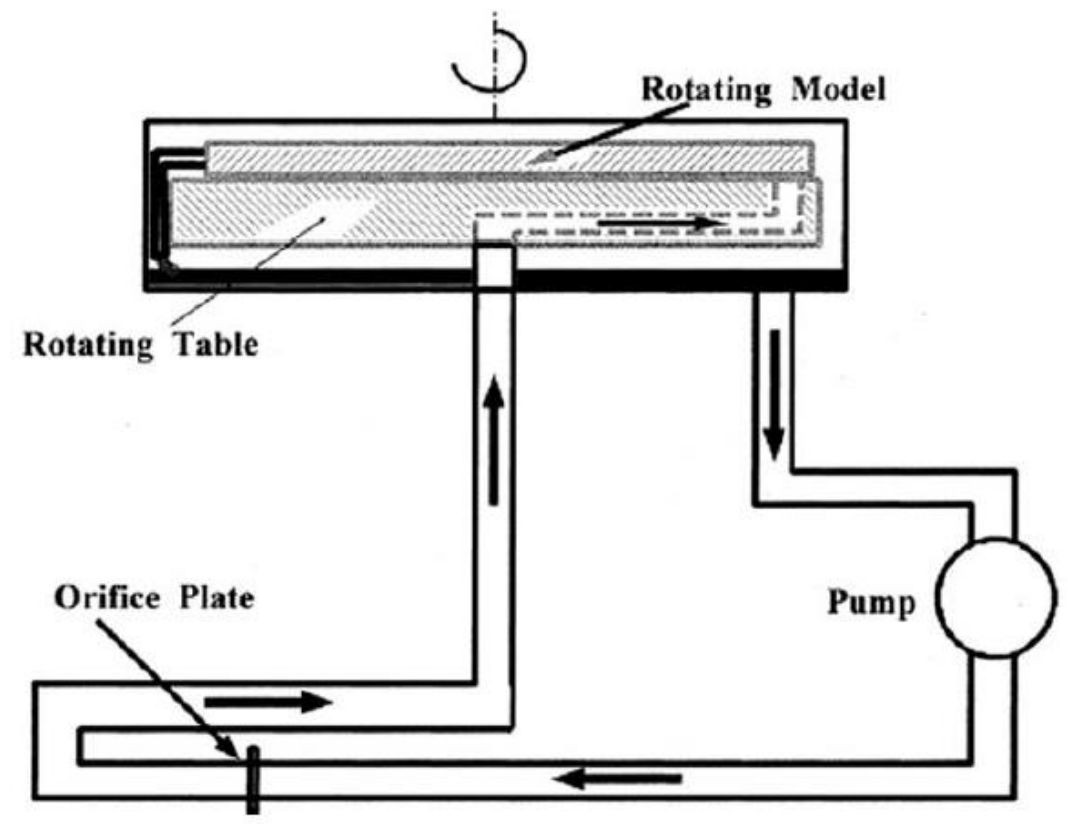

Figure 12. Experimental facility diagram [4]

The primary uncertainty with validation against this experiment is due to uncertainty about the model placement within the chamber. The passage total length is $740 \mathrm{~mm}$. the diameter of the chamber is 1.2 meters. Unfortunately, the specific orientation of the axis of rotation relative to the passage geometry is not specified in the documentation. This means that, at a given rotational speed, the rotational acceleration experienced at the bend cannot be precisely controlled to match the experiment.

The experimental operating point against which CFD is validated is experimental test 7 , described in Table 3

Table 3. Operating point used for validation against Iacovides experimental data

\begin{tabular}{|c|c|c|c|c|c|}
\hline $\begin{array}{c}\text { Reynolds } \\
\text { Number }\end{array}$ & $\begin{array}{c}\text { Inflowing leg } \\
\text { velocity }(\mathrm{m} / \mathrm{s})\end{array}$ & $\begin{array}{c}\text { Prandtl } \\
\text { Number }\end{array}$ & $\mathrm{T}$ in $\left({ }^{\circ} \mathrm{C}\right)$ & $\Omega(\mathrm{rad} / \mathrm{s})$ & $\begin{array}{c}\text { Rotation } \\
\text { Number }\end{array}$ \\
\hline 36,000 & 1.03 & 24.7 & 24.7 & 13.7 & 0.4 \\
\hline
\end{tabular}


Very high resolution sampling of wall Nusselt number and availability of Nusselt Number contours are the key benefits of this data set. Use of water as the working fluid, and uncertainty about model orientation relative to the axis of rotation are the primary drawbacks of the data set.

\subsection{Grid Independence Study}

Insufficient mesh resolution presents a possible source of error in any CFD analysis. The flow structures that are responsible for the performance of the system, in this case wall heat transfer, must be resolved to get an accurate result. A mesh that is too coarse to resolve these flow structures will artificially diffuse them, resulting in a poor prediction of wall heat transfer.

A grid independence study is carried out using the HOST geometry to evaluate mesh density requirements for the following simulations.

Simulations are performed on several meshes at varying resolution to delineate the necessary mesh resolution to capture all flow structures which drive wall heat transfer. Mesh resolution which is deemed adequate based on this comparison is used for all other simulation within this study.

In addition to comparing mesh resolution, it is of interest to compare heat transfer results achieved with different mesh types. Unstructured tetrahedral meshes with inflation layers are standard for application to complex geometries for industrial application of CFD. Block structured hexahedral meshes, on the other hand, present advantages in terms of reduced element count and improved simulation performance at the cost of vastly increased time spent generating mesh. Block structured mesh is typically reserved for simpler geometries or academic analysis of complex geometries.

To account for impact of mesh type and resolution on simulation results, several mesh resolutions of each type are generated for the HOST geometry with skewed turbulators The HOST geometry with skewed turbulators was chosen as the test case because the most complex flow is expected 
which includes all the key physics that drive heat transfer in a rotating passage. The set of meshes compared is summarized in Table 4. 
Table 4. Meshes included in grid sensitivity study

\begin{tabular}{|c|c|c|}
\hline Type & Element Count & Node Count \\
\hline Hex & $6 \mathrm{M}$ & $5.9 \mathrm{M}$ \\
\hline Hex & $17 \mathrm{M}$ & $18.3 \mathrm{M}$ \\
\hline Hex & $32 \mathrm{M}$ & $33.1 \mathrm{M}$ \\
\hline Hex & $50 \mathrm{M}$ & $51.0 \mathrm{M}$ \\
\hline Tet & $9.4 \mathrm{M}$ & $4.4 \mathrm{M}$ \\
\hline Tet & $27 \mathrm{M}$ & $12.5 \mathrm{M}$ \\
\hline Tet & $41 \mathrm{M}$ & $18.8 \mathrm{M}$ \\
\hline Tet & $63 \mathrm{M}$ & $15.5 \mathrm{M}$ \\
\hline
\end{tabular}

All block structured mesh was generated using the Gridpro meshing software. Unstructured meshes are generated using ICEM in combination with the Fluent mesher.

Postprocessing for heat transfer within the HOST program geometry is performed by monitoring integrated heat flux through each of the heated plates of the experiment. The heat transfer is then manipulated to calculate a normalized Nusselt number, $\mathrm{Nu} / \mathrm{Nu}_{\infty}$. $\mathrm{Nu}_{\infty}$ represents the Nusselt number expected for fully developed flow in a straight duct at the Reynolds number observed in the CFD. The procedure described in section 3.1.1.1 is applied to calculate normalized Nusselt number, $\mathrm{Nu} / \mathrm{Nu}_{\infty}$ throughout the passage.

Normalized Nusselt numbers of each plate provide a convenient means to analyze heat transfer throughout the passage. Figure 13 compares these results for each of the meshes included in the grid independence study. 


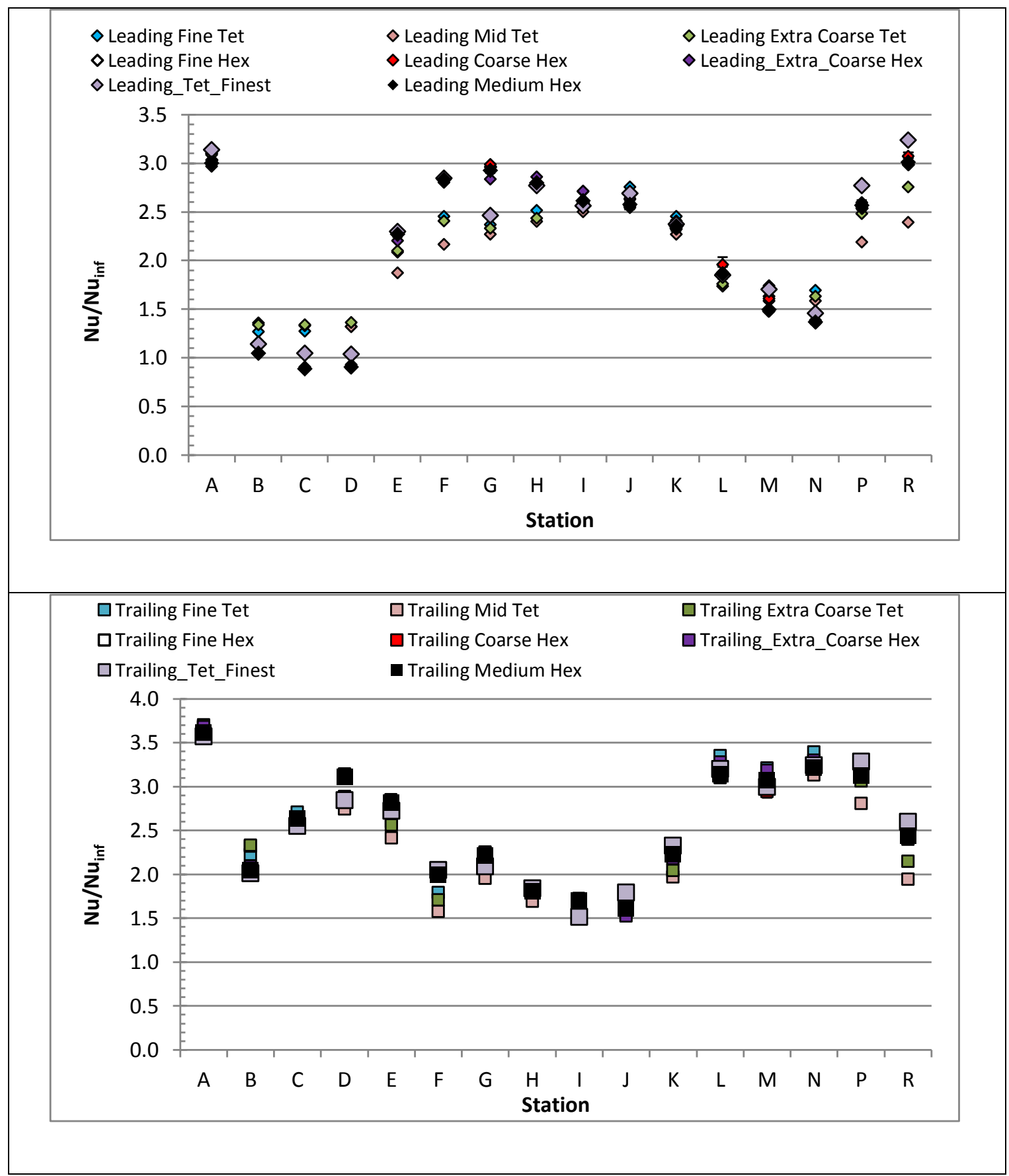

Figure 13. Leading and trailing wall Nusselt Number ratio for grid independence study

Small variations are observed in computational results between the various block structured meshes included in the study. The unstructured mesh results, however, vary significantly. The 'Tet Finest' data series approaches the behavior of the structured mesh results, but still differs significantly in some locations, especially station $\mathrm{G}$ on the leading endwall. 
At station $\mathrm{G}$, a vortex core is present which reattaches to the wall after the bend. The attachment point and path of the vortex core is significant to the local mesh resolution. Figure 14 and Figure 15 compare the nominal hex mesh results to the finest tetra mesh results at station G.

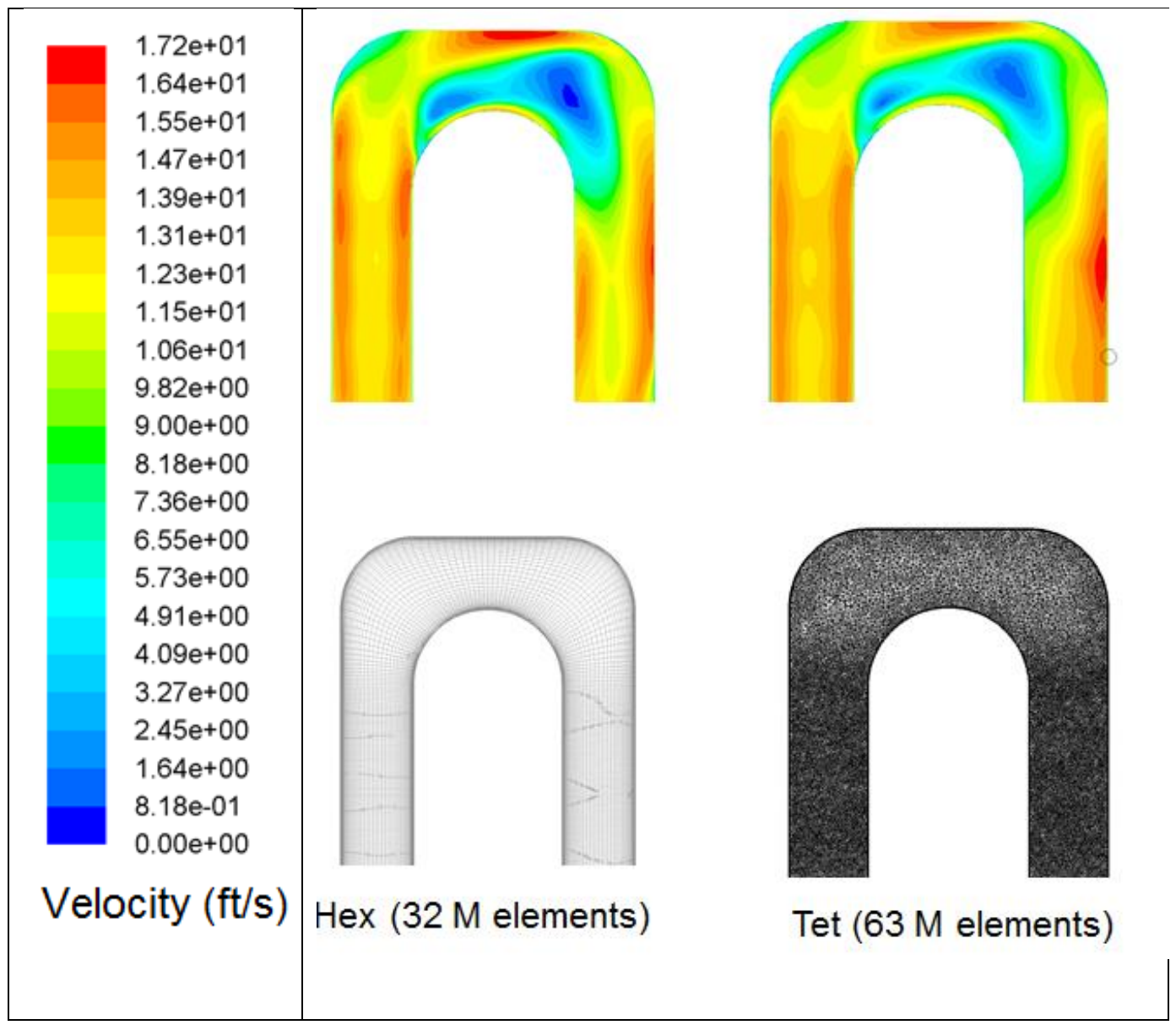

Figure 14. Velocity contours and Mesh at mid plane with element counts specified 


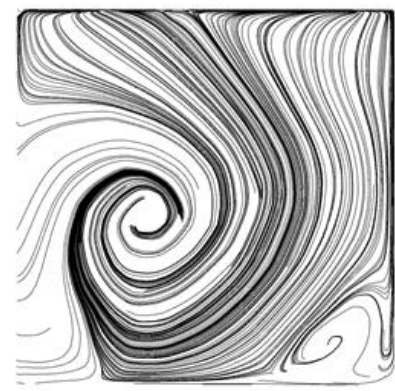

Tet mesh secondary flow

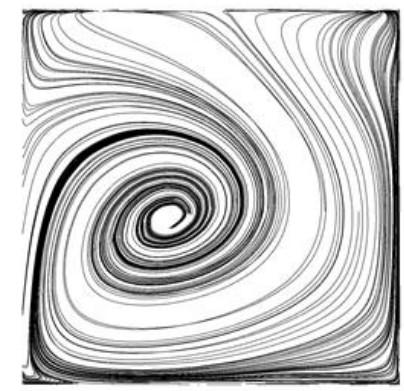

Hex mesh secondary flow

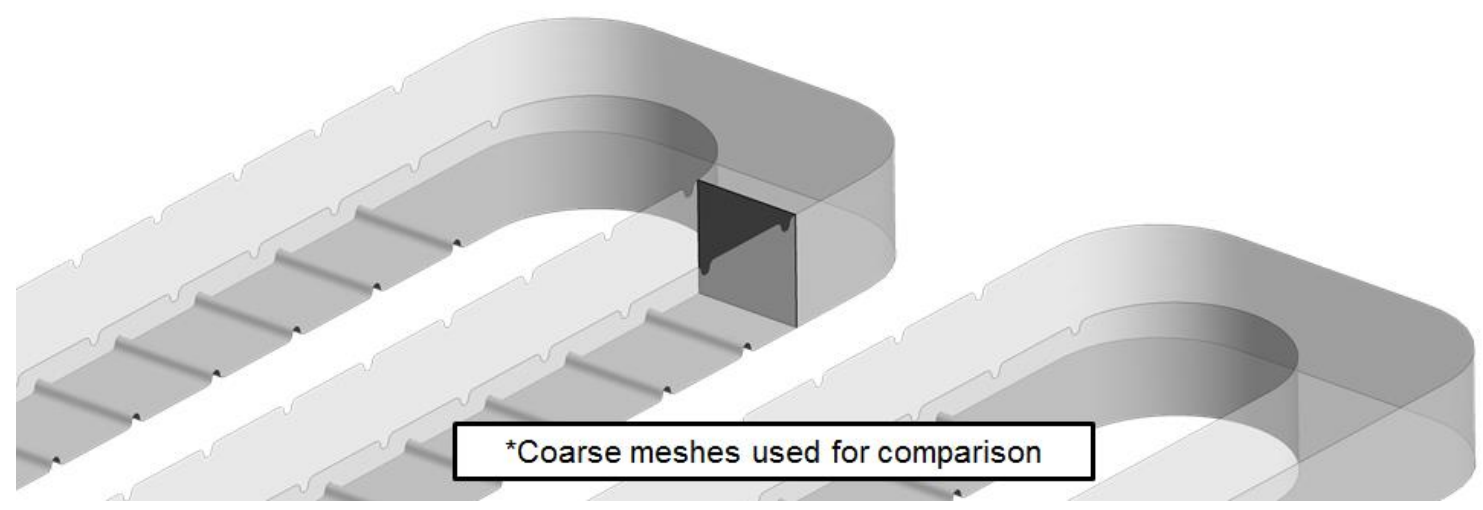

Figure 15. Comparison of secondary flow leaving the $1^{\text {st }}$ bend with different mesh types

The course hex mesh results in a strong vortex which is fully attached to the inner bend wall at the indicated location. In the Tet mesh, this vortex is accompanied by additional secondary vortices and separated from the inner wall. Because the behavior in the bend is clearly grid dependent on all simulated tetrahedral meshes, the differences between results in Figure 14 and Figure 15 is interpreted as an impact of insufficient mesh resolution to capture the generation and propagation of the secondary flows which determine flow behavior downstream of the bend.

A numerical summary of the grid independence study results is provided in Table 5. The $32 \mathrm{M}$ element hex mesh is chosen as the nominal hex mesh resolution, as it is shown to be grid independent. 
Table 5. Grid independence study results

\begin{tabular}{|c|c|c|c|c|}
\hline Type & $\begin{array}{c}\text { Element } \\
\text { Count }\end{array}$ & Node Count & $\begin{array}{c}\text { Average } \\
\text { plate error } \\
\text { (from nominal) }\end{array}$ & $\begin{array}{c}\text { Temperature } \\
\text { Out (F) }\end{array}$ \\
\hline Hex & $6 \mathrm{M}$ & $5.9 \mathrm{M}$ & $2.08 \%$ & 604.5 \\
\hline Hex & $17 \mathrm{M}$ & $18.3 \mathrm{M}$ & $1.05 \%$ & 604.5 \\
\hline Hex & $32 \mathrm{M}$ & $33.1 \mathrm{M}$ & $\mathrm{N} / \mathrm{A}$ & 604.5 \\
\hline Hex & $50 \mathrm{M}$ & $51.0 \mathrm{M}$ & $1.01 \%$ & 604.4 \\
\hline Tet & $9.4 \mathrm{M}$ & $4.4 \mathrm{M}$ & $9.02 \%$ & 603.5 \\
\hline Tet & $27 \mathrm{M}$ & $12.5 \mathrm{M}$ & $15.32 \%$ & 603.3 \\
\hline Tet & $41 \mathrm{M}$ & $18.8 \mathrm{M}$ & $8.38 \%$ & 603.4 \\
\hline Tet & $63 \mathrm{M}$ & $15.5 \mathrm{M}$ & $5.10 \%$ & 603.8 \\
\hline
\end{tabular}

The chosen mesh approach for the host validation study based on the $32 \mathrm{M}$ element mesh in

Table 5 is summarized below. Figure 16 illustrates the typical mesh resolution in the turbulated region.

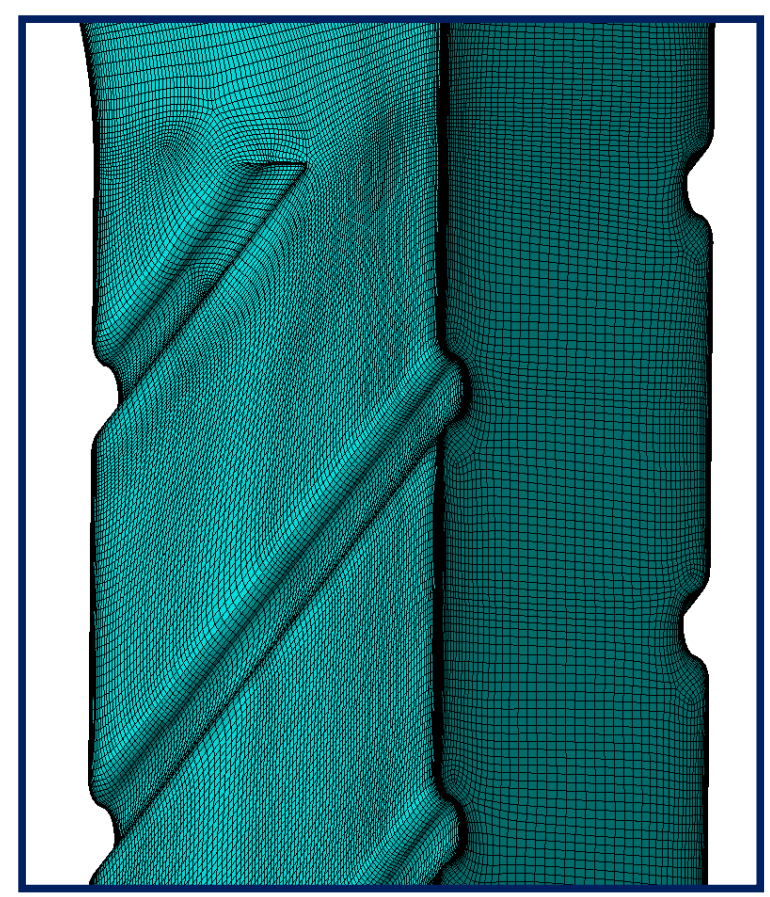

Figure 16. Nominal mesh resolution in turbulated region 
Another important consideration in terms of mesh is boundary layer resolution. In order to model low-Reynolds near wall behavior, either with $\mathrm{k}-\varepsilon$ enhanced wall functions or $\mathrm{k}$ $\omega \mathrm{SST}$, it is important to have sufficient elements within the near wall region of the boundary layer. A $1^{\text {st }}$ element wall spacing y+ below 1 is desirable. Figure 17 shows the $y+$ range observed in the turbulated baseline simulation.

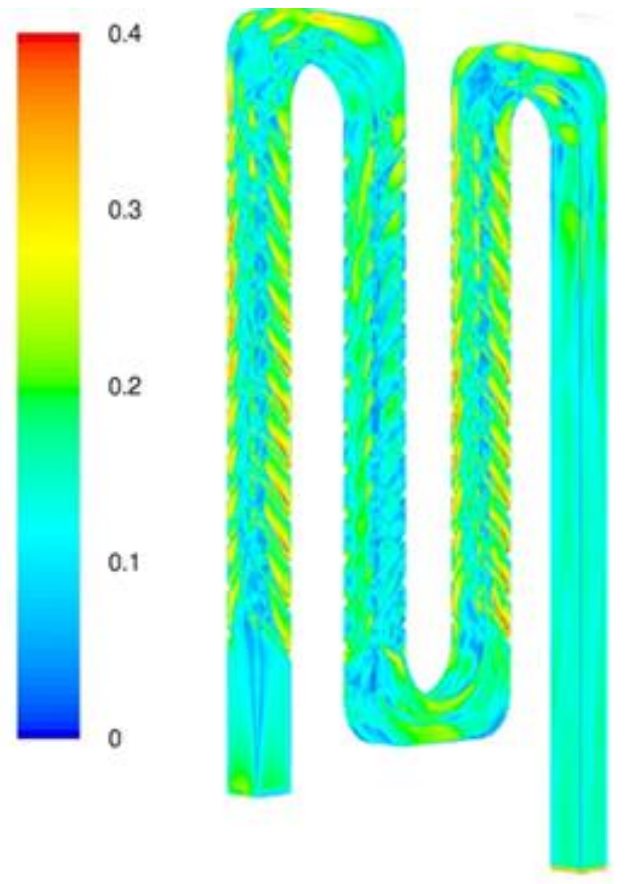

Figure 17. y+ contours for rotating turbulated baseline simulation

The contours of $y+$ shown are from turbulated results for the rotating baseline operating condition. The maximum $y+$ of 0.87 occurs near the exit boundary, and the area averaged $\mathrm{y}+$ is 0.165 .

When enhanced wall functions are applied, Fluent treats the region where the turbulent Reynolds number is below 200 as viscosity affected. The solver then blends from the near wall, low Reynolds model to the high Reynolds model. It is important to have several elements along this blend, with many elements in the viscosity affected region. 
Figure 18 shows 38 elements in the viscosity affected region and 5 elements along the layer blend.

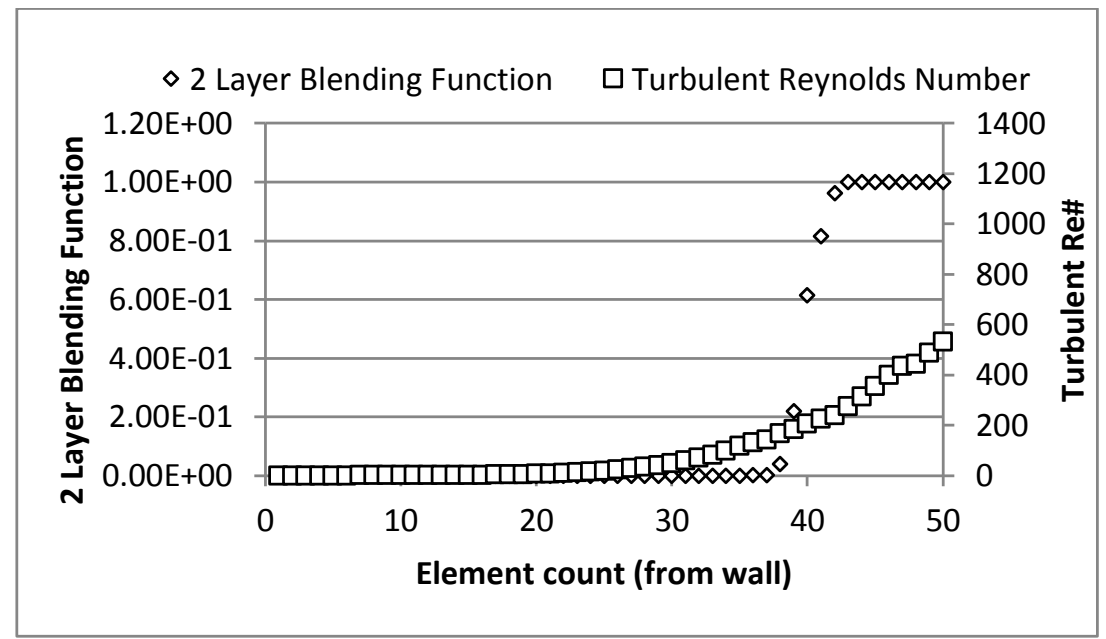

Figure 18. Boundary layer mesh properties

This data is collected normal to the wall at a location with average $y+$ compared to the bulk of the domain. The boundary layer is highly resolved in this analysis. Boundary layer meshing requirements are well understood. For the RANS modelling performed in this analysis, the boundary layer resolution in the utilized mesh is ideal.

The final meshes used for HOST program validation and turbulence modelling study are listed in Table 6. Any results presented for the HOST geometries in upcoming sections are simulated on these meshes. The non-turbulated mesh consists of much fewer elements as there is no need to resolve turbulator-induced flow structures. 
Table 6. Summary of HOST validation study meshes

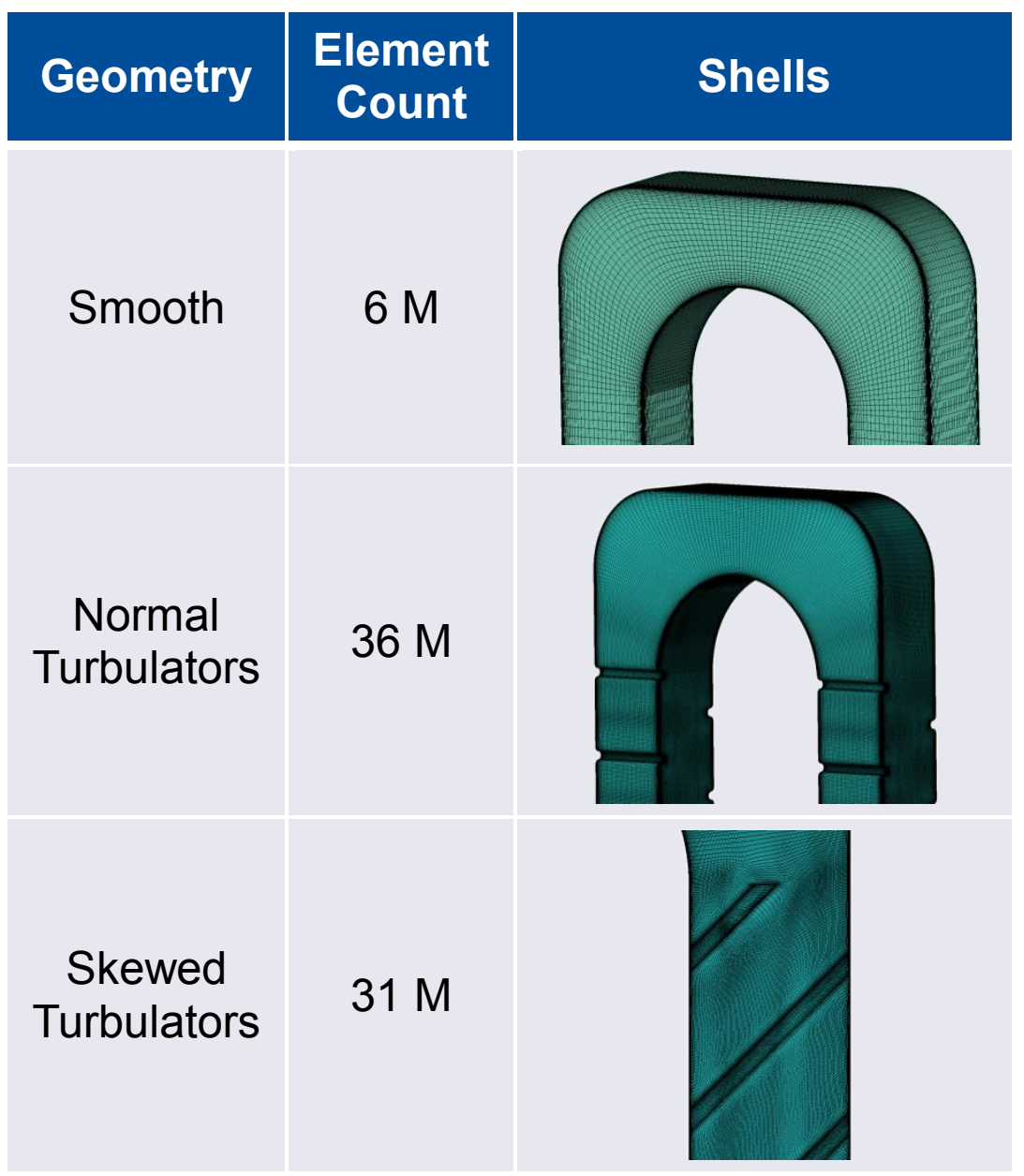

\subsection{Model Selection and Calibration}

Boundary conditions, geometric simplifications, and numerical models influence CFD results. This section describes the process undertaken to decide on each of these issues and create the most effective CFD model possible. The HOST program geometry and data were used to conduct this study.

\subsubsection{Plenum Entrance Effect}


Flow quantities at the simulation inlet significantly impact heat transfer in the first leg of duct. The HOST experiment included a feed tube providing air to a plenum upstream of the test article with a filter to condition the flow.

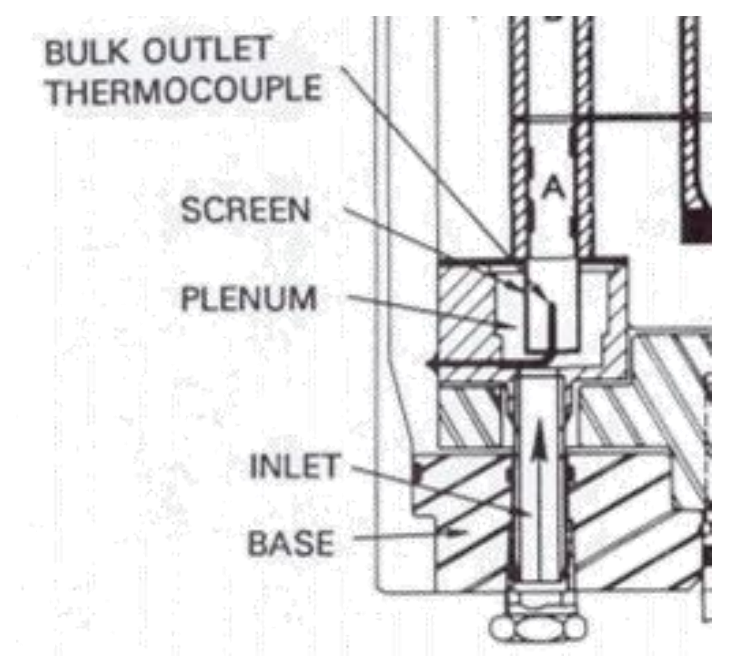

Figure 19. Experiment inlet configuration [2]

The plenum region is not included in the CFD model. A specific description of the plenum geometry and screen is not included in experiment documentation. Instead, experimental measurements were taken to characterize the velocity and turbulence intensity profiles at the passage inlet as illustrated in Figure 20. 


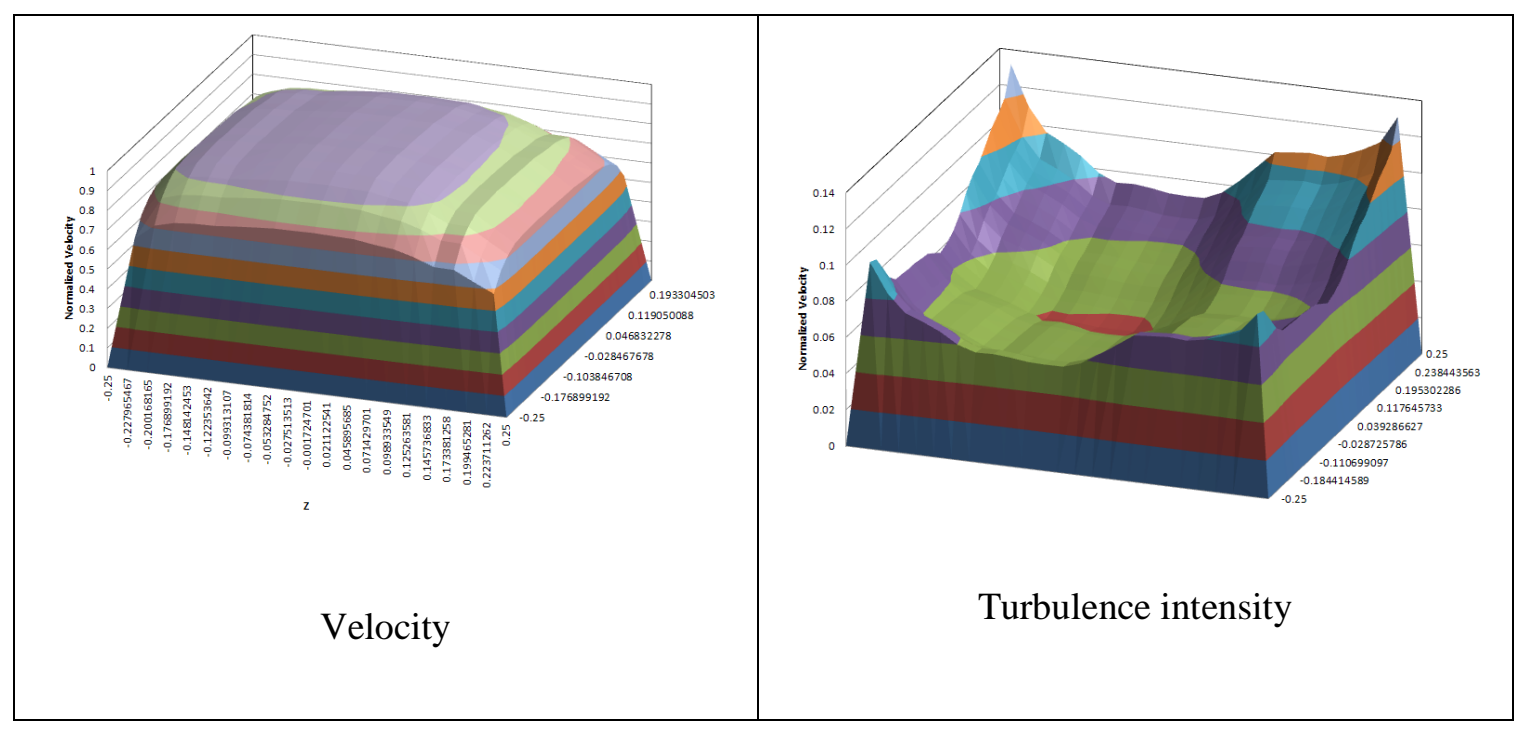

Figure 20. Experimentally measured inlet profiles

This data was collected for stationary, non-rotating operation during the HOST experiment. The resolution of the profiles applied at the inlet is determined by the resolution of the data collected during the HOST experimentation. The test data inlet profile is fairly coarse, resulting in a sudden jump at the boundary. The resolution of the CFD mesh to which these profiles are applied is much finer, as shown in Figure 21.

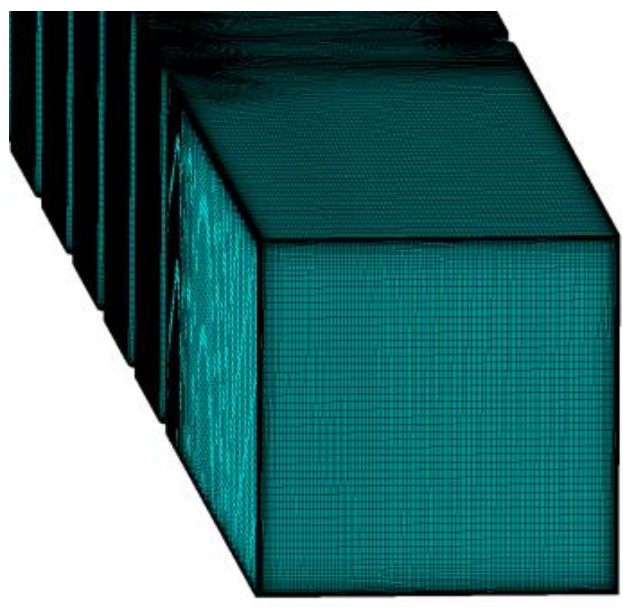

Figure 21. Entrance mesh resolution 
The impact of mapping these profiles to the CFD model was checked by comparing to a simulation which used a flat velocity profile. Figure 22 compares the heat transfer in the first leg between experiment, correlation, and CFD. Heat transfer is shown for the nonturbulated, stationary, baseline case.

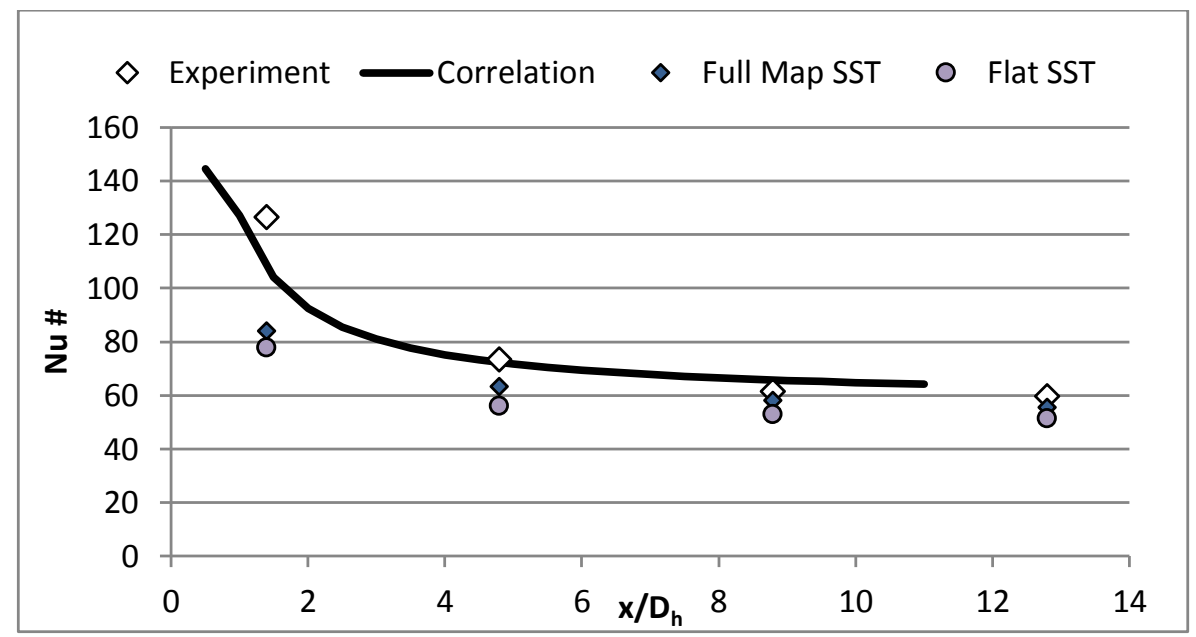

Figure 22. Entry region heat transfer

When compared to the experimental results, the CFD prediction of heat transfer at the duct entrance was consistently low. An entrance effect correlation was also applied, which predicted heat transfer between the experimental and computational prediction. The "Full Map" results in Figure 22 correspond to CFD simulation with the experimental profiles applied. The results for the full passage, including all legs is shown in Figure 25. 


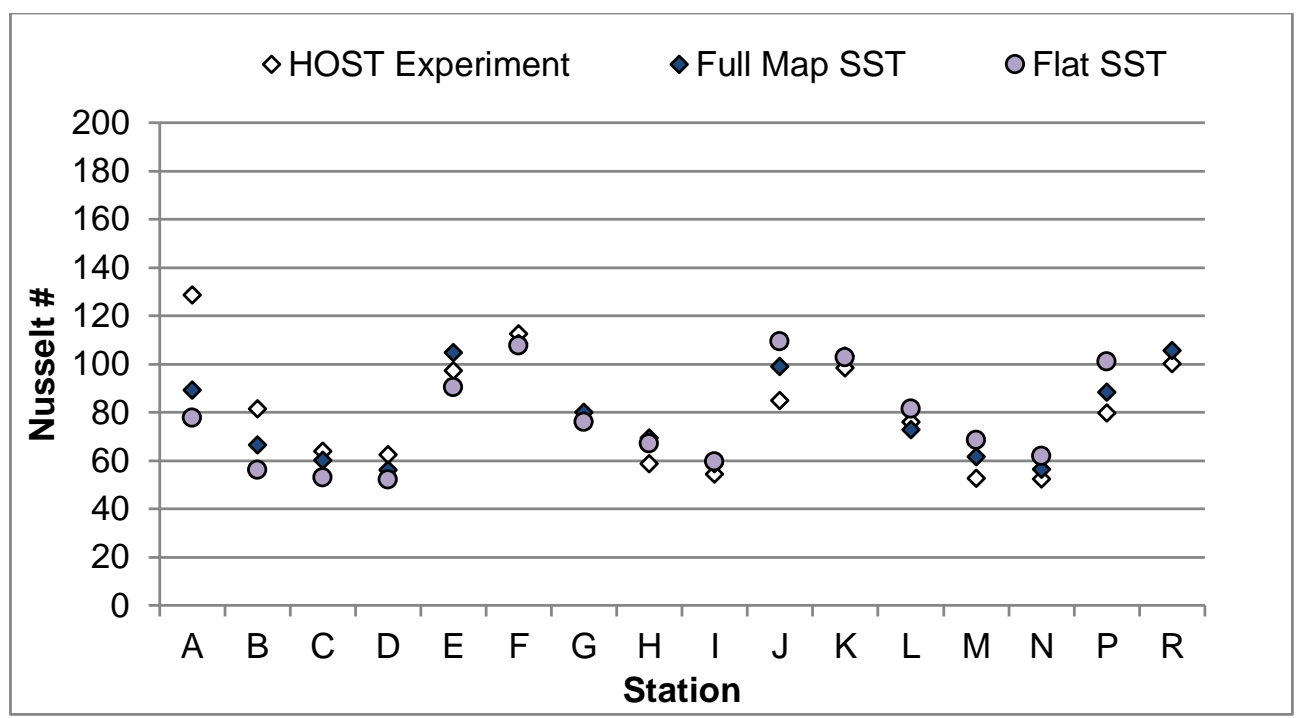

Figure 23. Full passage heat transfer with varying inlet condition

The impact of the applied profiles does impact the CFD result, but the deviation from experiment near the entrance is much greater than the difference between the sets of CFD results. Note that flow in the bends is subject to instability, and will be discussed in upcoming sections. The mapped inlet condition is used for upcoming analyses because it represents the most comprehensive boundary condition available for validation. Rotating simulations use a constant turbulence intensity inlet because the inlet profile is measured for a non-rotating case and does not represent the turbulence magnitude of rotating operation. $5 \%$ turbulence intensity and a 0.035 " length scale are applied. Inlet velocity is mapped for all HOST CFD cases.

\subsubsection{Chamfer Impact}

Small chamfers in the corners of HOST duct are indicated in the NASA documentations

[2]. These chamfers are created when the wall plates are pressed together and are roughly $1 \mathrm{~mm}$. Chamfer Geometry is illustrated in Figure 24. 


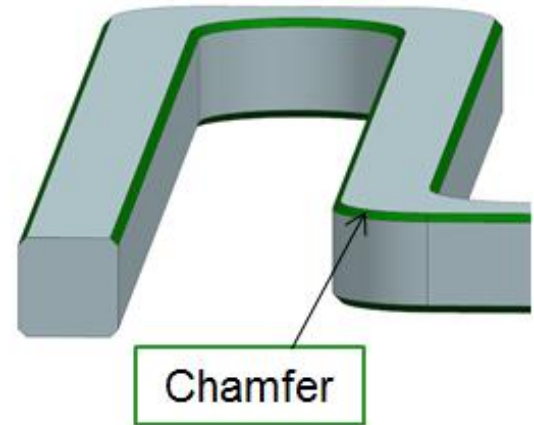

Figure 24. Chamfer Geometry

Mesh generation is greatly simplified by neglecting these chamfers. Heat transfer throughout the duct, with and without chamfers, for the non-turbulated, rotating, baseline operating point is compared in Figure 25.

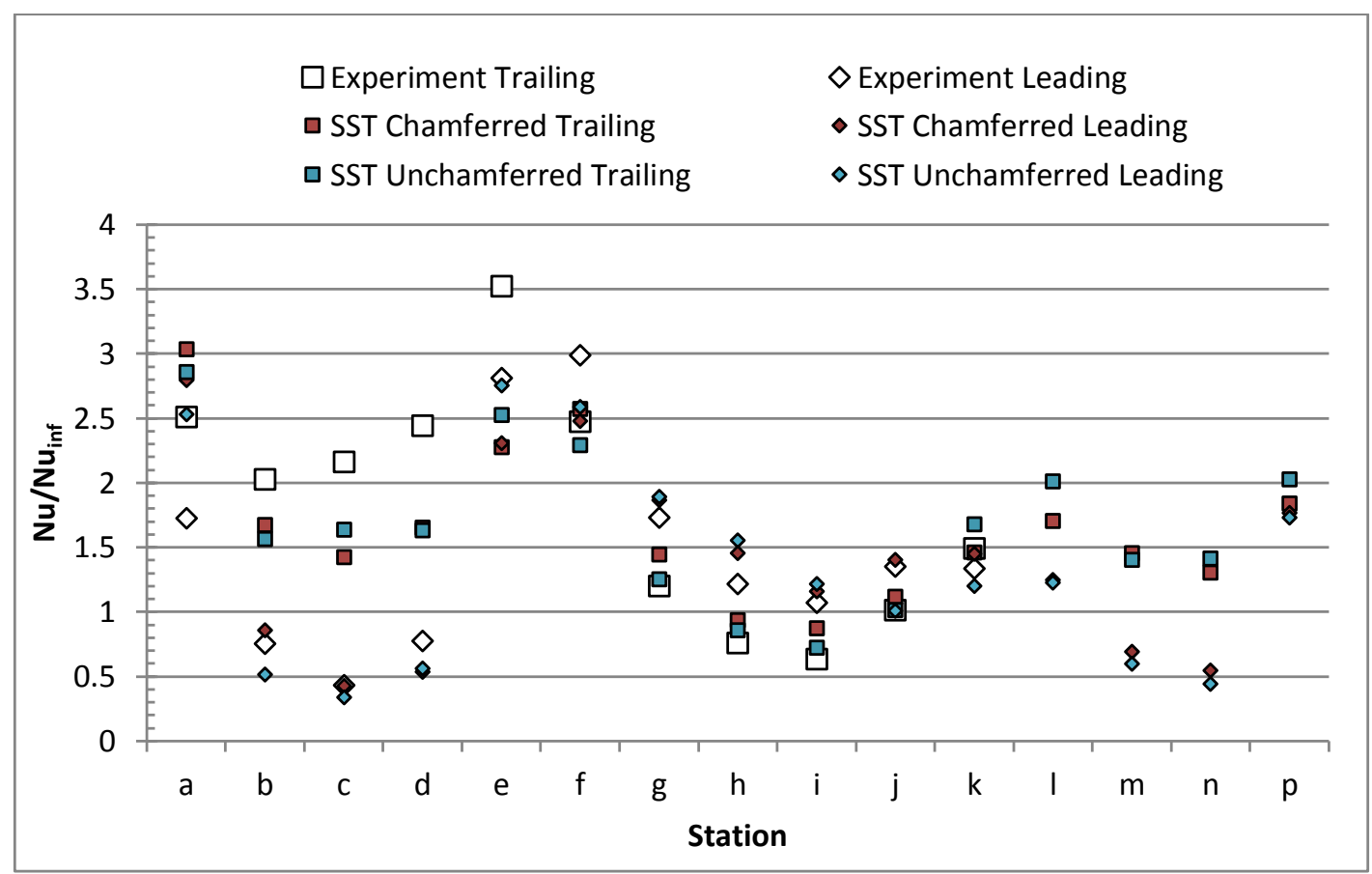

Figure 25. Heat transfer impact of chamfer

In the entry leg and the entrance to the first bend, the impact of the chamfer is notable.

Throughout the majority of the passage, the impact is below $10 \%$ and within the margin expected for CFD analysis of such a complex flow. Resolution of the chamfers 
throughout this study would come at the cost of increased time and effort required, reduced mesh quality, and increased element count. Inclusion of the chamfer is out of scope of the current effort considering the magnitude of the impact.

\subsubsection{Simulation convergence and Physical unsteadiness}

The physical phenomena experienced within rotating internal cooling passages result in complex, unsteady flow. Destabilized flow in the bends separates, resulting in unsteady turbulent structures which impact heat transfer. Additionally, the Coriolis driven flow is known to destabilize the boundary layer unstable at the trailing wall. [2]

Physical unsteadiness in the problem being solved manifests in steady-state CFD simulation as solution oscillations or apparent poor convergence. The integrated heat flux through the separate plates is monitored as the CFD solver iterates. The variation in heat transfer throughout the final 5000 iterations of the rotating baseline case with skewed turbulators is illustrated in Figure 26.

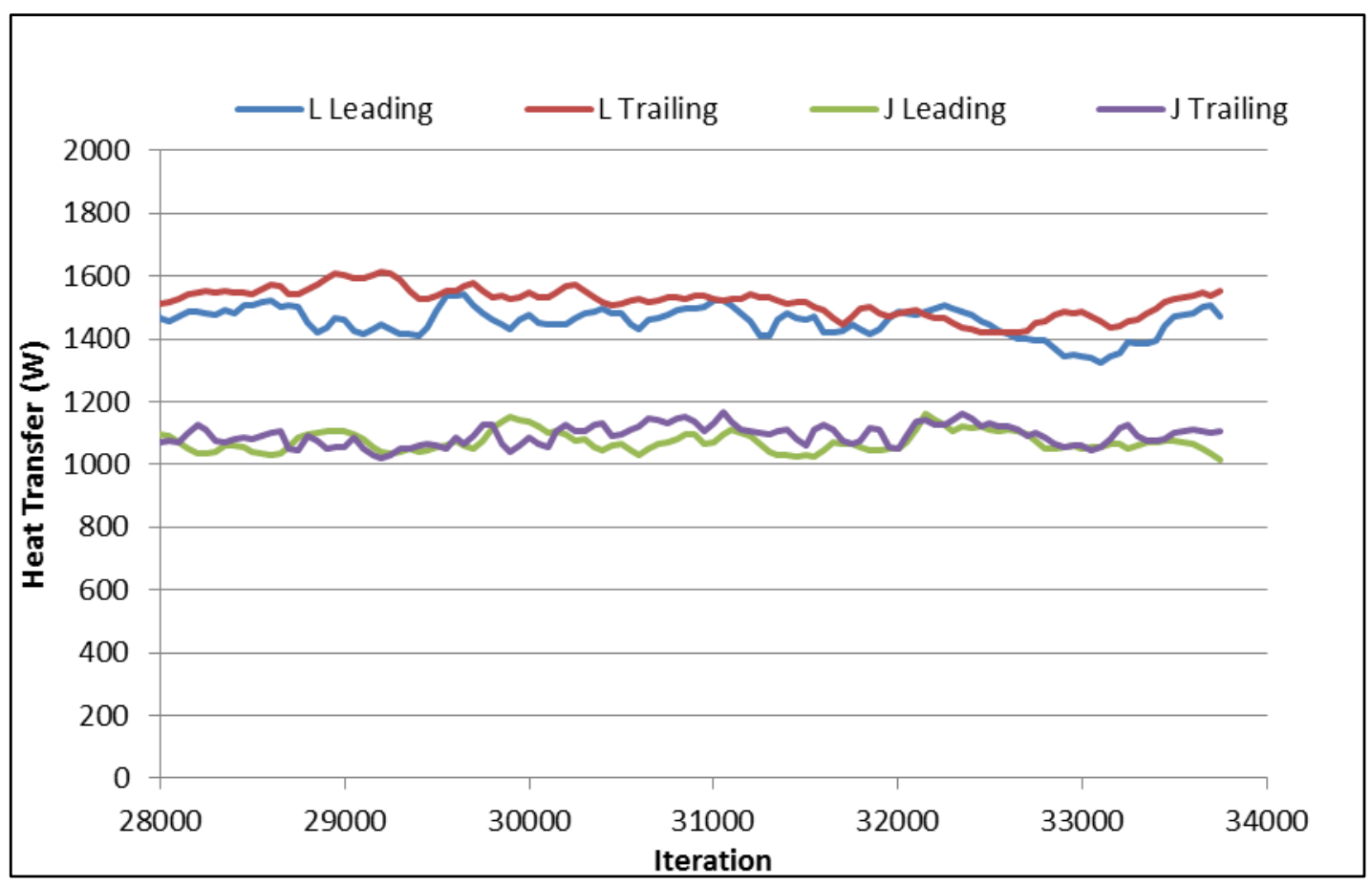

Figure 26. Solution oscillation with iteration 
The monitors shown correspond to the inner bend, which exhibits high relative instability compared to the rest of the domain. When steady state results are used to report the heat transfer through these plates, the result is representative of a snapshot at that specific iteration, without regard for the oscillations present. Error bars based on min and max values observed in the last 5000 iterations are plotted along with the steady state CFD solution and experimental data for the non-rotating baseline smooth walled case. The bend regions and regions further downstream exhibit significant variation.

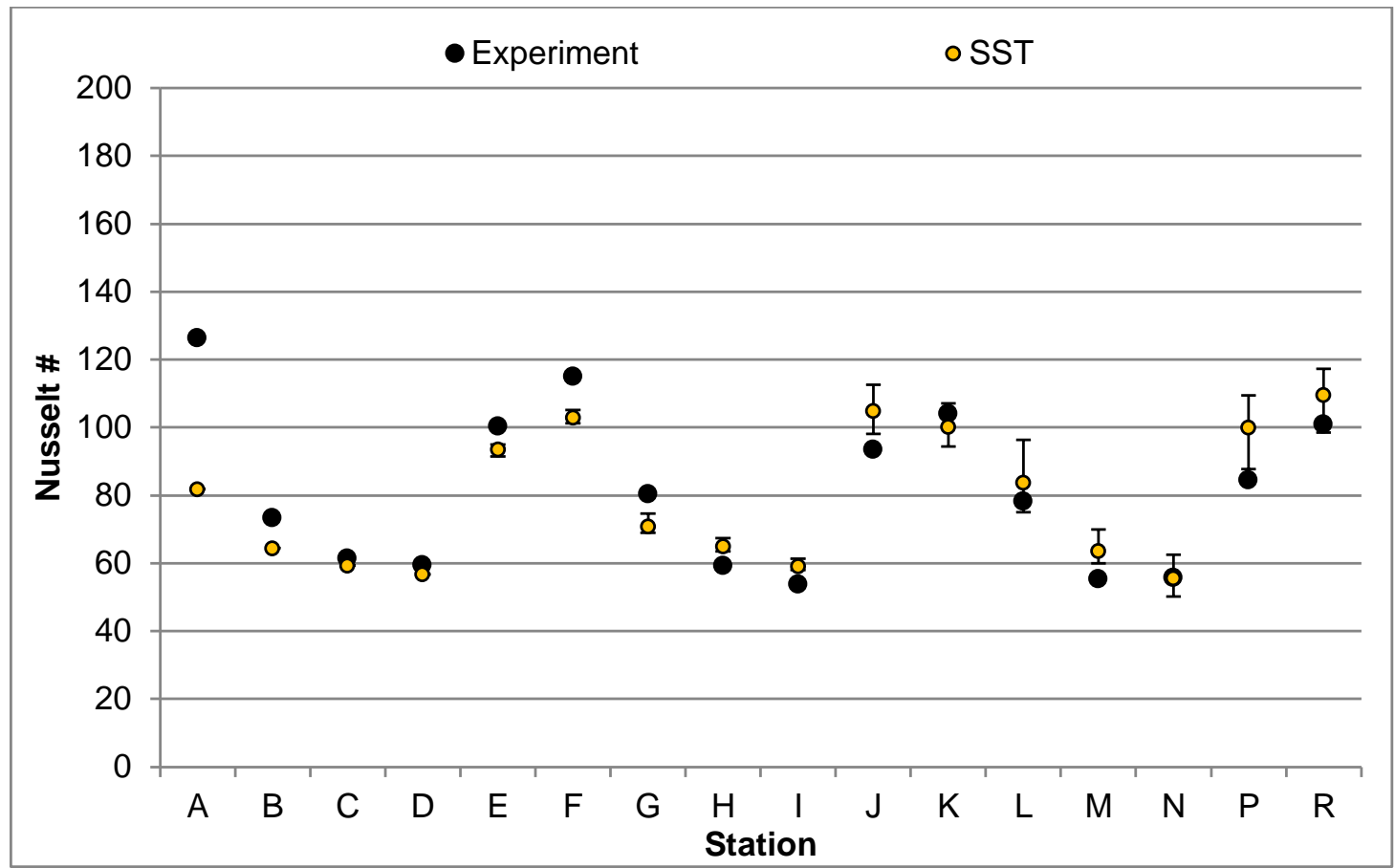

Figure 27. Heat transfer variation by station along the HOST passage

Note that the error bars are generated using the minimum and maximum value of each plate Nusselt number within the most recent 5000 iterations.

Transient CFD which explicitly resolves unsteady behavior in time is computationally expensive in terms of storage space and run time. URANS SST and SAS-SST were run to evaluate the impact of a time averaged unsteady solution compared to a steady state snapshot solution. 
A compromise between time averaging and steady state results is implemented to filter out noise without incurring the costs of full unsteady simulation. Heat flux is monitored through each plate in the passage and recorded, as shown in Figure 26. The heat flux of each plate is then arithmetically averaged over the most recent 5000 iterations, in a process referred to from here on as "pseudo-time averaging." This is not equivalent to a true time average, but serves to filter out some of the solution instability. True unsteady simulation advances between timesteps then executes an inner loop to converge the updated flowfield. This process accounts for the unsteady transport of conservation properties in a timeaccurate way that pseudo time averaging does not.

Steady state "snapshot" results, pseudo-time averaged results, and unsteady time averaged results are compared for the baseline rotating simulation in Figure 28

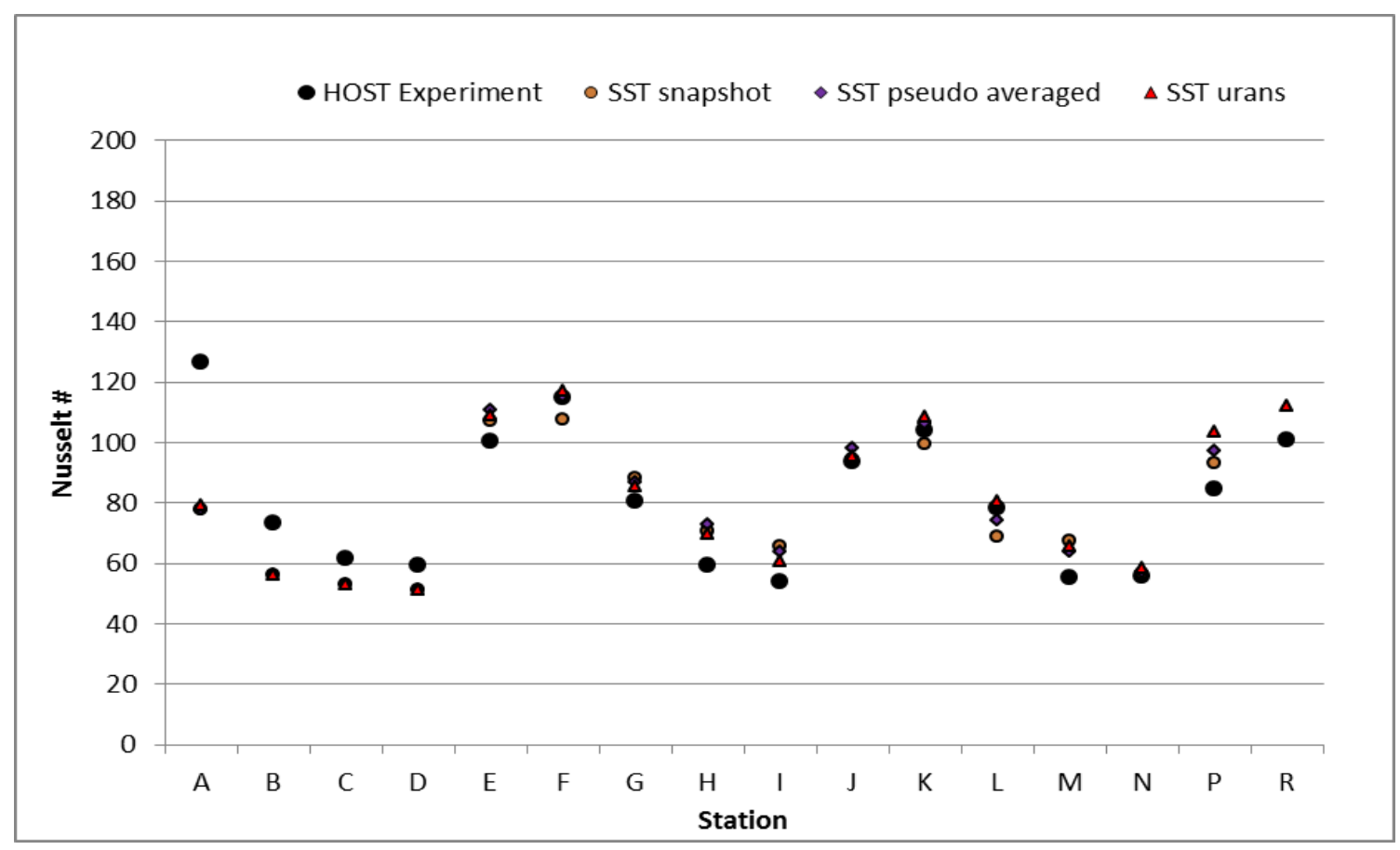

Figure 28. Steady state, pseudo averaged, and time averaged Nu\# on leading endwall at stationary baseline condition 
The pseudo averaged results follow the full unsteady results and experimental results more faithfully, with fewer outliers. At later stations, especially, the consistency of the results is improved significantly by filtering out unsteadiness with pseudo time averaging. The URANS dataset included is a time accurate solution with time averaging performed by Fluent.

\subsubsection{Turbulence Modelling}

As discussed, the behavior of fluid within a rotating serpentine passage is driven by a combination of complex flow phenomena. The ability of different turbulence models to capture different aspects of these physical phenomena becomes an important question to address. Several available turbulence models are applied to the stationary and rotating operation of the HOST passage to evaluate performance.

Realizable $\mathrm{k}-\varepsilon$, or RKE is a variation of the of the $\mathrm{k}-\varepsilon$ model which mathematically removes one of the correlation constant inherent to standard k- $\varepsilon$. k- $\varepsilon$ has been an industry standard 2 equation model for many years. Advantages are high industry familiarity and stability. Prediction of separation and reattachment, however, is relatively poor using RKE. Separation plays a large role in the internal cooling passage so RKE performance may be non-ideal.

SST or shear stress transport is another 2 equation turbulence model which blends between

k- $\omega$ near the wall, and k- $\varepsilon$ in the domain away from the walls. Fluent and CFX SST based calculations are performed to ensure consistency in setup and repeatability of the simulation.

RSM, or the Reynolds Stress Model is also included in this study. The advantage of RSM is that it represents turbulence viscosity anisotropically. $\mathrm{k}-\omega$ and $\mathrm{k}-\varepsilon$ each use two transport equations to compute a scalar value of turbulence viscosity, in accordance with the 
Boussinesq hypothesis. As a scalar, this viscosity is effectively isotropic, or acting the same in all directions. In reality turbulence is not always isotropic. For example, near a wall, turbulence fluctuations normal to the wall are suppressed relative to those along the wall. As indicated in section 2.1, capturing turbulence anisotropy allows RSM to capture features such as corner vortices that develop in a square duct.

Disadvantages to RSM are relative instability, and a lack of common adoption within industry.

The final model considered is SAS, or scale adaptive simulation. SAS is a modification to $\omega$ based models which adds a source term based on local mesh resolution [6]. The Fluent theory guide describes SAS as an "improved URANS formulation which allows the resolution of the turbulent spectrum in unstable flow conditions."

Heat transfer along the passage as predicted by the various turbulence models is illustrated in Figure 29 for the stationary operating point. Note that smooth wall geometry is used for comparison of turbulence models unless otherwise specified. 


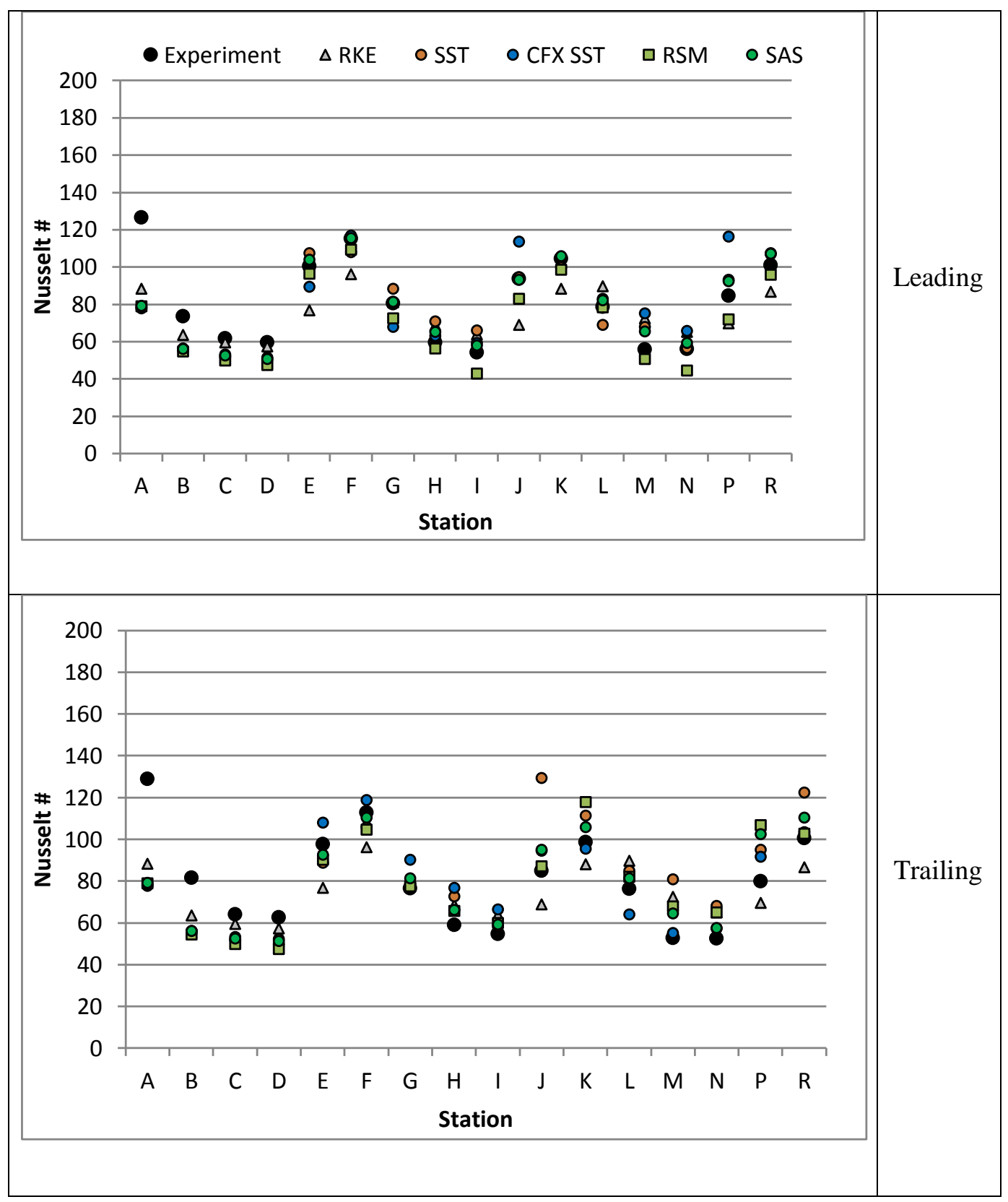




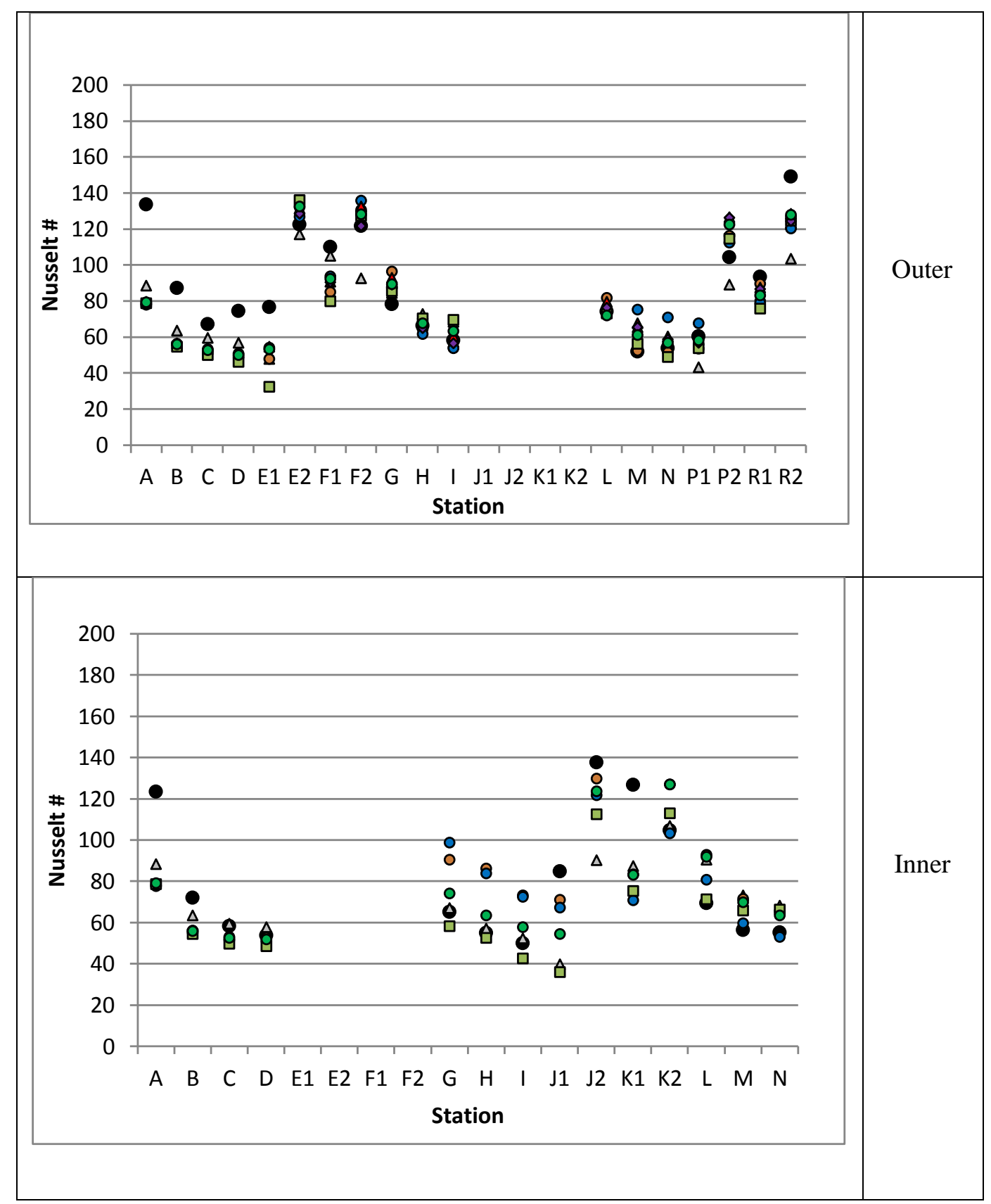

Figure 29. Passage Nusselt Number predicted with various turbulence models

RKE is the clear outlier in the heat transfer calculations in Figure 29. RKE demonstrates consistently low heat transfer relative to experiment. However, RKE demonstrates more stable convergence and a cleaner, more consistent trend than the other models. A more qualitative comparison of results is included in Figure 30, which illustrates wall shear predicted by various turbulence models. 


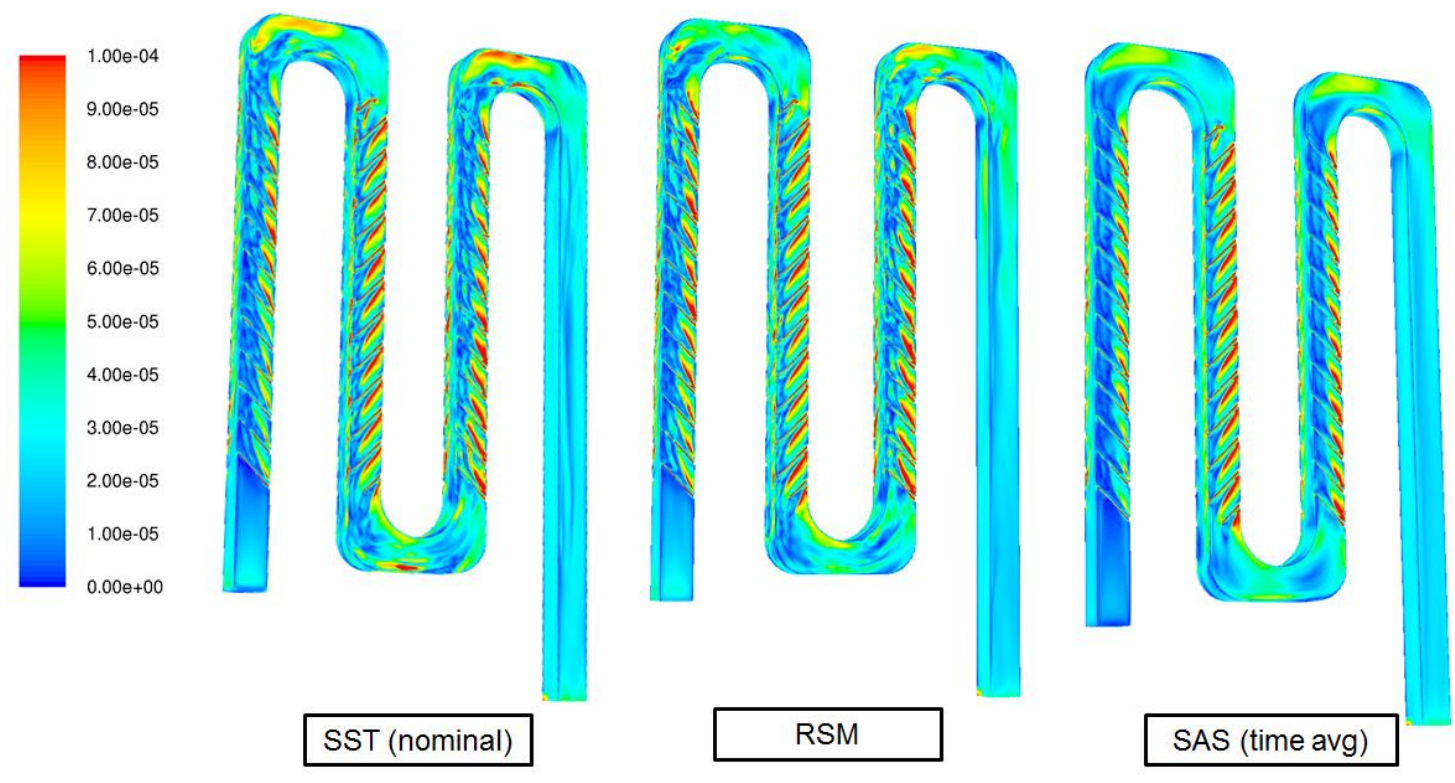

Figure 30. Wall shear contours from various turbulence models (psi)

SST and RSM contours are instantaneous at a given time step. Noise appears in the separated, turbulent regions. Qualitatively, the degree of separation between SST and RSM results varies. SAS results are shown on a time averaged basis. Generally, wall shear behavior is consistent across all 3 turbulence models. Physical phenomena such as anisotropy and high frequency turbulence, which are resolved by RSM and SAS, respectively, do not appear to dominate flow behavior in the passage. 
The heat transfer calculated by the various CFD turbulence models is compared in Figure

31 for the rotating baseline operating point.

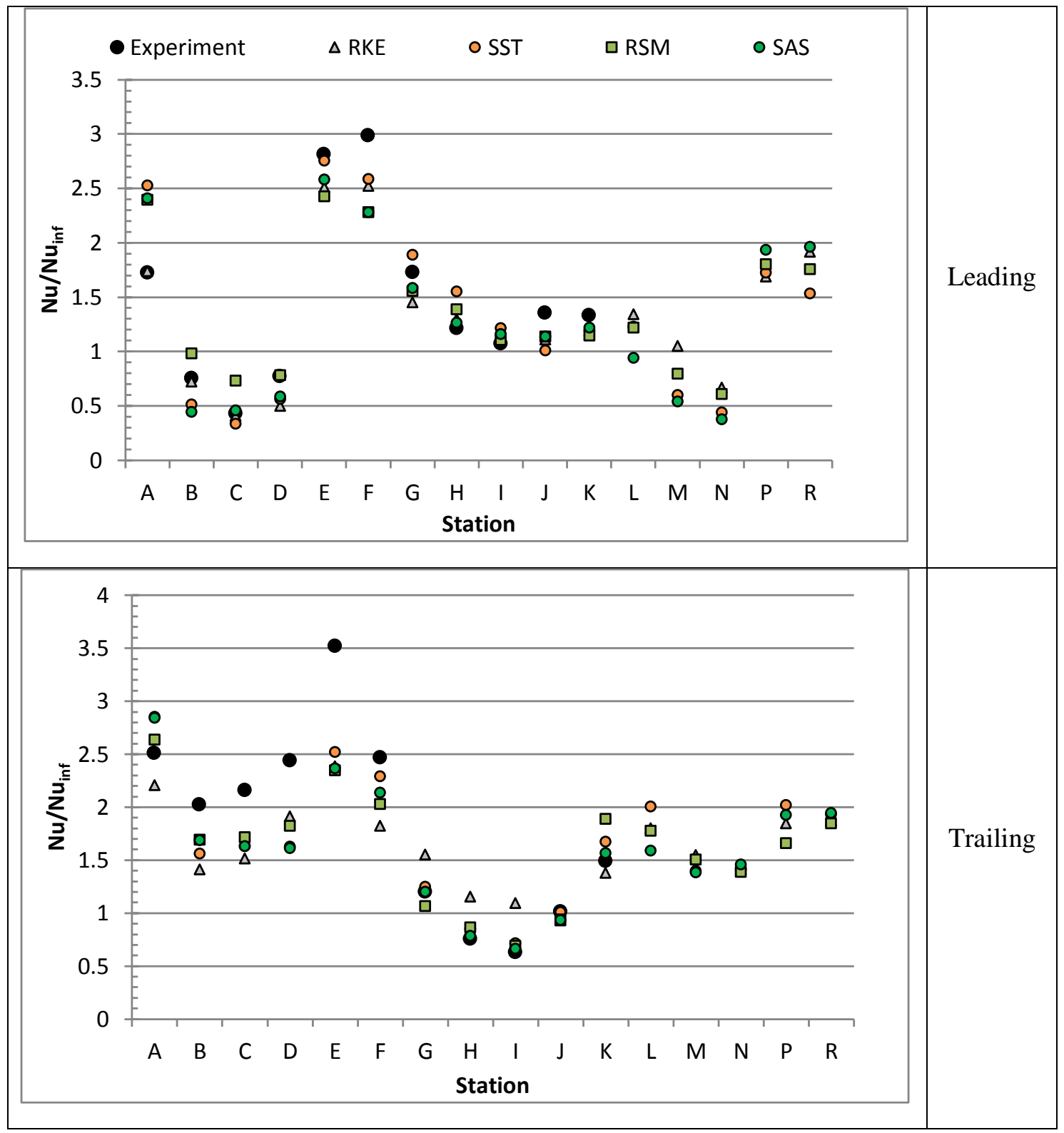




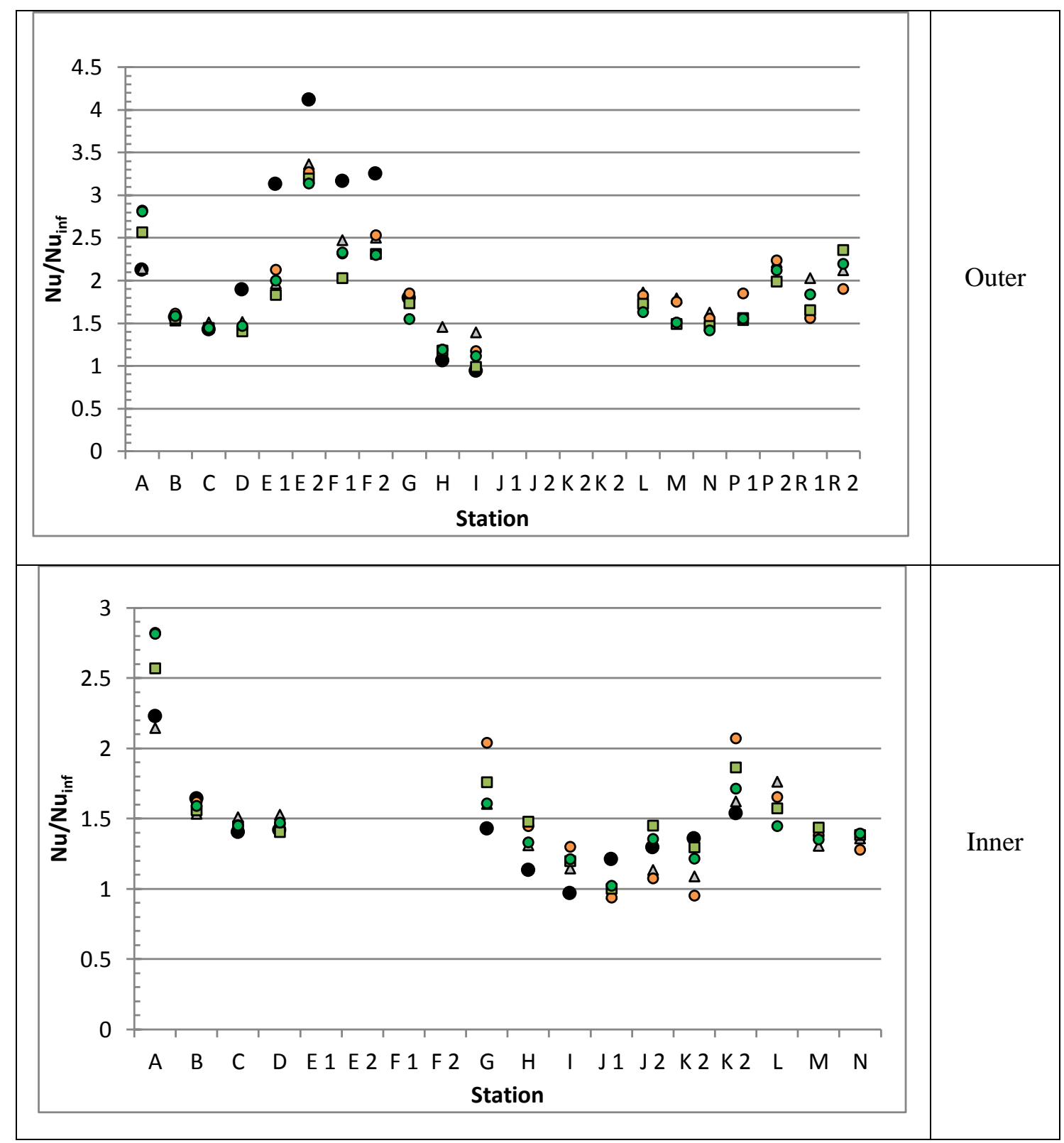

Figure 31. Nusselt Number Ratio predicted by various turbulence models for rotating baseline operating point

A summary of the deviation between CFD and experiment for the studied CFD models is presented in Table 7. 
Table 7. Error from experiment for Turbulence model study

\begin{tabular}{|c|c|c|c|c|}
\hline \multirow{2}{*}{ Model } & \multicolumn{2}{|c|}{ Stationary } & \multicolumn{2}{c|}{ Rotating } \\
\cline { 2 - 6 } & $\begin{array}{c}\text { Avg } \% \\
\text { error }\end{array}$ & $\begin{array}{c}\text { RMS } \% \\
\text { error }\end{array}$ & $\begin{array}{c}\text { Avg } \% \\
\text { error }\end{array}$ & $\begin{array}{c}\text { RMS } \% \\
\text { error }\end{array}$ \\
\hline RKE & -10.5 & 16.4 & -2.94 & 18.3 \\
\hline SST & -4.23 & 20.6 & N/A & N/A \\
\hline SST AVG & -5.66 & 18.6 & -0.885 & 19.3 \\
\hline SST CFX & -6.11 & 20.3 & N/A & N/A \\
\hline RSM & -14.8 & 19.6 & -0.287 & 17.6 \\
\hline SAS & -8.17 & 16.1 & -4.102 & 15.8 \\
\hline
\end{tabular}

"Avg error" indicates an arithmetic average which is equivalent to the average in the total heat flux of the passage. RMS error accounts for local deviations from experiment. "SST Avg" indicates results are averaged in pseudo-time using the most recent 5000 iterations of SST results.

The RMS error is increased for $\omega$ based models which capture a greater degree of separation and accompanying unsteadiness in solution. These models, however, match the experiment much better in terms of average \% error. This means that the omega based models exhibit local peaks and valleys that are not present in the experimental data, but follow the experimental trend in a bulk sense. RKE results in a smoother trend, but under-predicts total passage heat transfer.

The results for the leading wall with various models are further compared to experiment in Figure 32. The 45 degree trendline represents a perfect match between CFD and the experimental data. 


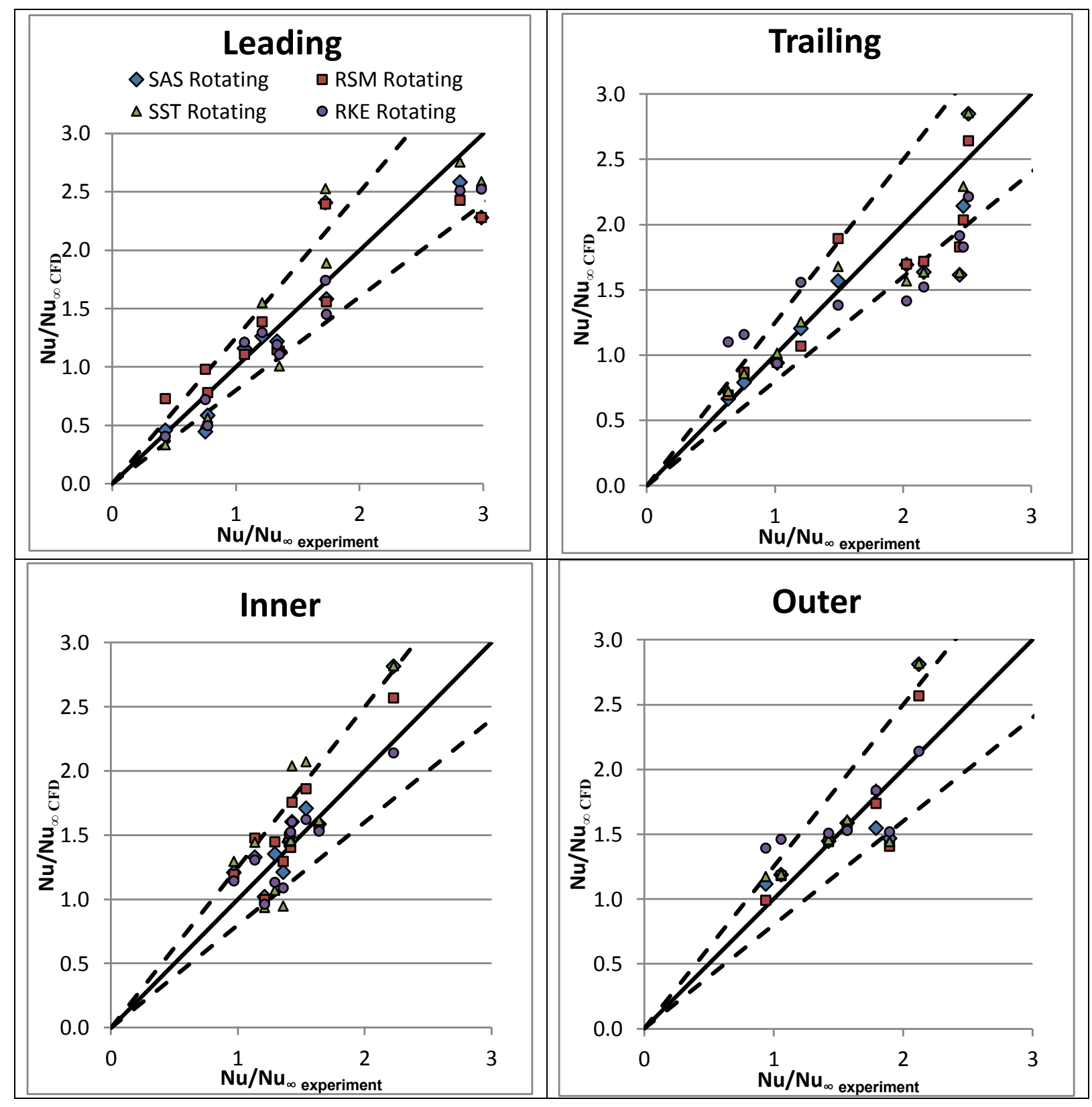

Figure 32. Experiment comparison to CFD 45 degree plot

By plotting each data point with experimental $\mathrm{Nu} / \mathrm{Nu}_{\infty}$, error is represented as deviation from a 45 degree line. $\pm \% 20$ variation between CFD and experiment is marked with dashed lines. The entrance region leading into the $1^{\text {st }}$ bend accounts for most of the outliers present. The SST model with pseudo time averaging is chosen for use in the computational model 
due to a low average percent error with a level of noise filtering to reduce the impact of physical unsteadiness.

\subsubsection{Model Selection Conclusions}

The exercises outlined in this section were used to calibrate a modelling setup and approach which best balances simulation accuracy, computational cost, Industry acceptance of models, and intuition to model the physics of internal cooling flow. Error in CFD analysis is generally categorized as discretization error, roundoff error, convergence error, and modelling error. The exercises performed in this model study allow the rough quantification of these various sources of error, summarized in 8 .

Table 8. CFD error and uncertainty breakdown

\begin{tabular}{|c|c|c|}
\hline Category & $\begin{array}{l}\text { Method of evaluation or } \\
\text { reasoning }\end{array}$ & Assessed error \\
\hline Discretization & Mesh sensitivity study & $\sim 1 \%$ for nominal hex mesh \\
\hline Round-off & $\begin{array}{c}\text { Double precision computing with } \\
\text { moderate aspect ratios and } \\
\text { variations }\end{array}$ & Negligible \\
\hline $\begin{array}{l}\text { Iterative / } \\
\text { convergence }\end{array}$ & $\begin{array}{c}\text { Flow unsteadiness evaluation and } \\
\text { averaging }\end{array}$ & $\begin{array}{l}\sim 2 \% \text { for } 1 \text { up-pass increasing } \\
\text { to } 15 \% \text { in final bend }\end{array}$ \\
\hline \multirow{3}{*}{$\begin{array}{c}\text { Modelling / } \\
\text { Approximation }\end{array}$} & Inlet Condition study & $\begin{array}{c}\sim 50 \% \text { for } 1 \text { st } \text { section, } \sim 10 \% \text { for } \\
\text { second, }>5 \% \text { further } \\
\text { downstream }\end{array}$ \\
\hline & Chamfer Simplification study & $\begin{array}{l}\text { Up to } 8 \% \text { locally in bend } 1, \\
\text { generally }<5 \%\end{array}$ \\
\hline & Turbulence model selection & $<20 \%$ observed error \\
\hline
\end{tabular}

The primary source of concern with respect to model accuracy is the level of variation from experiment observed in the turbulence model sensitivity study. Rotating passage flow is 
characterized by interaction of several viscous phenomena which is difficult to capture using off the shelf turbulence modelling. Note that decoupling error due to turbulence model selection alone from other sources of error is not immediately possible. Reported error for turbulence model selection includes impacts of all other error sources.

By investigating model sensitivity to various settings and inputs, an educated model selection is made possible. The model setup converged on for use in future simulations is summarized in Table 9.

Table 9. Simulation setup used for Final Simulations

\begin{tabular}{|cc|}
\hline & Value \\
\hline Solver & Fluent 14.5 \\
\hline Wall treatment & Enhanced \\
\hline Fluid & Air: incompressible Ideal Gas \\
\hline Discretization & Second Order \\
\hline Pressure-Velocity Coupling & SIMPLE \\
\hline Buoyancy & Non-Boussinesq \\
\hline Rotation & Frame Motion \\
\hline Turbulence Model & SST \\
\hline Curvature Correction & On \\
\hline Flitering & Pseudo Time Averaging, 5000 iterations \\
\hline Monitors & Not Included \\
\hline Chamfers & Mapped Velocity, TKE, and $\omega$ \\
\hline Stationary & Mapped Velocity, 5\% Turbulence Intensity, \\
\hline Rotating & 0.07 Dh length scale \\
\hline Inlet Mapping
\end{tabular}

The applied material properties of air are temperature dependent. Polynomial fits for dynamic viscosity, thermal conductivity, and specific heat are applied with respect to temperature. 


\subsection{HOST Validation}

The modelling setup and approach developed in the preceding sections is evaluated against the HOST data throughout a suite of operating points with variations in various non-dimensional parameters. For the set of experimental operating points validated, see Table 10.

Table 10. HOST validation test suite

\begin{tabular}{|c|c|c|c|c|c|c|c|}
\hline Case & $\begin{array}{c}\text { Re } \\
\text { Number }\end{array}$ & $\begin{array}{c}\text { Ro } \\
\text { Number }\end{array}$ & $\begin{array}{c}\text { Radius } \\
\text { Ratio } \\
\text { (R/d) }\end{array}$ & $\begin{array}{c}\text { Rotation } \\
\text { Angle }\end{array}$ & $\begin{array}{c}\text { Inlet } \\
\text { Density } \\
\text { Ratio }\end{array}$ & Turbulators & Description \\
\hline 1 & 25,000 & 0 & 49 & 0 & 0.13 & None & \multirow{3}{*}{$\begin{array}{l}\text { Baseline } \\
\text { Stationary }\end{array}$} \\
\hline 2 & 25,000 & 0 & 49 & 0 & 0.13 & Straight & \\
\hline 3 & 25,000 & 0 & 49 & 0 & 0.13 & $45 \mathrm{deg}$ & \\
\hline 4 & 25,000 & 0.24 & 49 & 0 & 0.13 & None & \multirow{3}{*}{$\begin{array}{l}\text { Baseline } \\
\text { Rotating }\end{array}$} \\
\hline 5 & 25,000 & 0.24 & 49 & 0 & 0.13 & Straight & \\
\hline 6 & 25,000 & 0.24 & 49 & 0 & 0.13 & $45 \mathrm{deg}$ & \\
\hline 7 & 25,000 & 0.34 & 49 & 0 & 0.13 & None & \multirow{2}{*}{$\begin{array}{l}\text { Effect of } \\
\text { Rotation } \\
\text { Number }\end{array}$} \\
\hline 8 & 25,000 & 0.34 & 49 & 0 & 0.13 & $45 \mathrm{deg}$ & \\
\hline 9 & 25,000 & 0.24 & 49 & 0 & 0.07 & None & \multirow{4}{*}{$\begin{array}{c}\text { Effect of } \\
\text { Density Ratio }\end{array}$} \\
\hline 10 & 25,000 & 0.24 & 49 & 0 & 0.23 & None & \\
\hline 11 & 25,000 & 0.24 & 49 & 0 & 0.07 & $45 \mathrm{deg}$ & \\
\hline 12 & 25,000 & 0.24 & 49 & 0 & 0.23 & $45 \mathrm{deg}$ & \\
\hline 13 & 12,500 & 0.12 & 49 & 0 & 0.13 & None & \multirow{4}{*}{$\begin{array}{l}\text { Effect of } \\
\text { Reynolds } \\
\text { Number }\end{array}$} \\
\hline 14 & 50,000 & 0.12 & 49 & 0 & 0.13 & None & \\
\hline 15 & 12,500 & 0.12 & 49 & 0 & 0.13 & 45 deg & \\
\hline 16 & 75,000 & 0.12 & 49 & 0 & 0.13 & $45 \mathrm{deg}$ & \\
\hline
\end{tabular}

Heat transfer experimental and CFD results are compared primarily in terms of total passage heat pick up, Plate Nusselt number, Plate Nusselt number ratio, \% deviation from experiment, and \% deviation from baseline operating point. 


\subsubsection{Baseline Results}

Simulations 1-6 represent baseline operation and are consistent with the grid independence and turbulence model comparison studies. Other operating points typically vary from these baseline conditions by varying one of the non-dimensional parameters used to describe the physics of rotating passages.

The flowfield through the passage is illustrated using volume renderings and streamlines looking toward the leading suction side endwall. The volume rendering is a 3-D representation of the flow with opacity increasing with velocity and colored by velocity. The flow inlet is the leftmost leg base as shown.

The nonrotating results with smooth walls, Case 1, are shown in Figure 33.

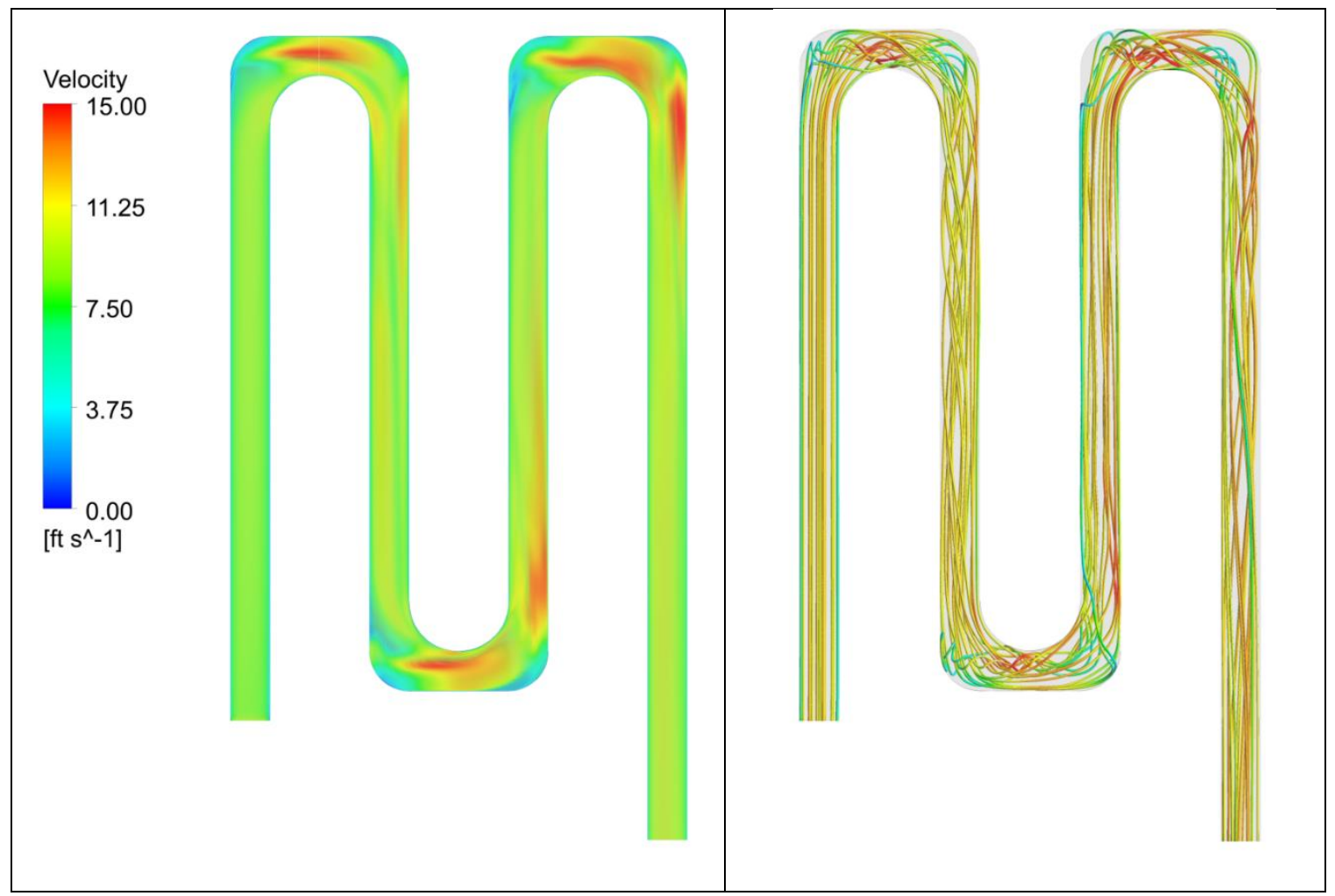

Figure 33. Side view of passage flow for non-rotating smooth HOST geometry (Case 1)

Secondary flows in the form of dean vortices are generated by the bend, but the flow is otherwise calm, with little crossflow. 
The nonrotating baseline case with skewed turbulators (Case 3) is shown in Figure 34.

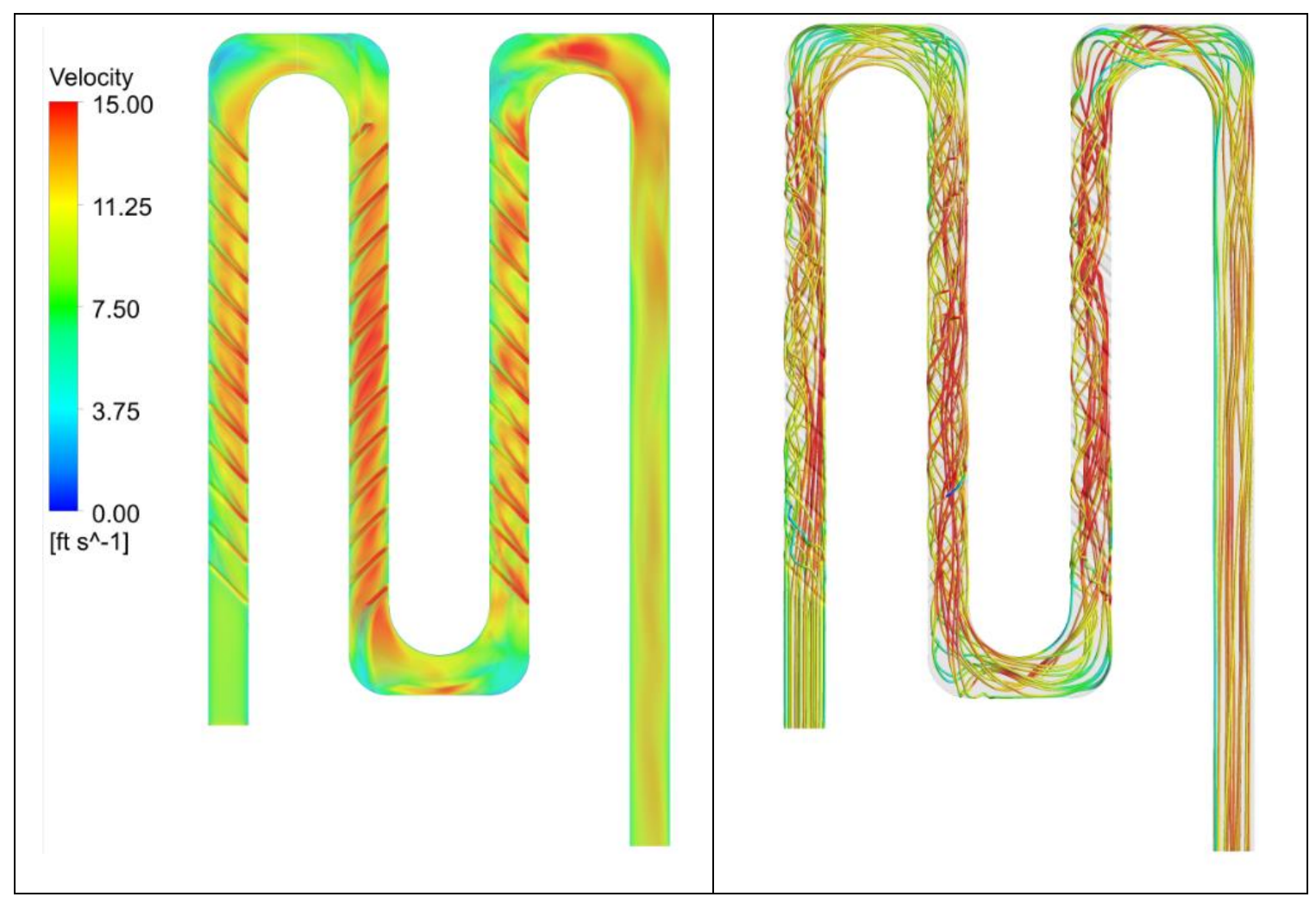

Figure 34. Velocity visualization viewed toward leading end wall

The addition of skewed turbulators generates secondary flows in the straight leg sections. Additionally, flow is concentrated, resulting in regions of higher velocity than is observed in the non-turbulated results.

Results for the rotating baseline case with skewed turbulators are illustrated in Figure 35 


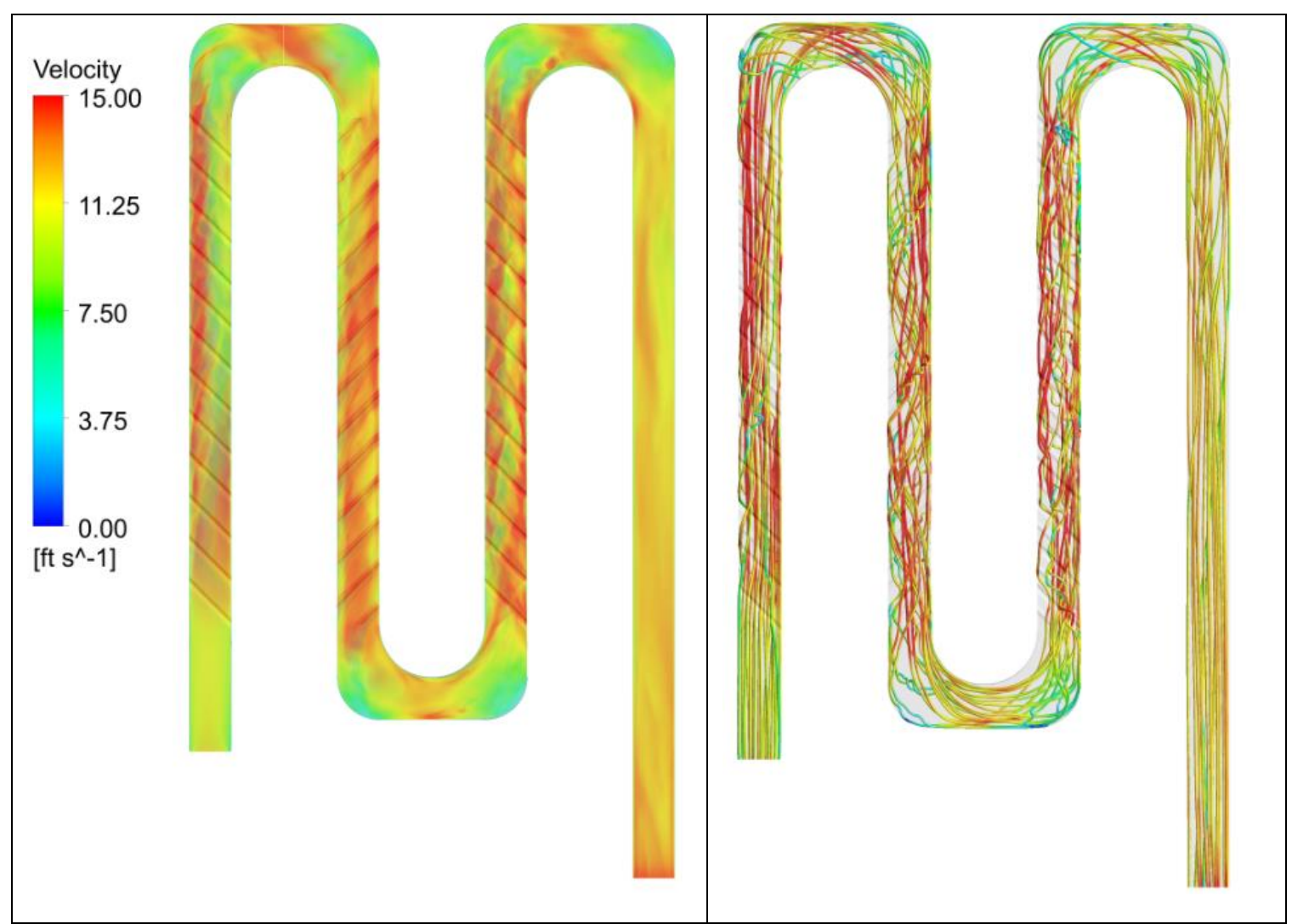

Figure 35. Case 6 Velocity Side View

In the bends, vortices carry high velocity flow toward the leading endwall. The flow is visibly less clean and uniform than in stationary operation, with passage rotation creating regions of high and low velocity near the wall in locations that were clean and uniform for the non-rotating case. 


\subsubsection{Passage Heat Pickup}

Validation of the total heat transfer of the entire heated section of the duct is performed in this section. The total heat transfer is computed by summing the plate heat transfer, Q, across all the heated plates in the passage. In this comparison, good agreement is achieved between the CFD model and experimental data. Table 11 compares the net heat pick up of the first two passes for each of the operating points simulated.

Table 11. Net passage heat transfer from CFD and experiment (Btu/h)

\begin{tabular}{|c|c|c|c|}
\hline & Qt,exp & Qt,cfd & Diff \\
\hline St. smooth & 468.12 & 454.49 & $2.91 \%$ \\
\hline St. skewed & 607.65 & 624.52 & $2.78 \%$ \\
\hline Rot. Smooth & 502.73 & 474.82 & $5.55 \%$ \\
\hline Rot. Straight & 557.73 & 572.41 & $2.63 \%$ \\
\hline Rot. Skewed & 606.22 & 609.95 & $0.62 \%$ \\
\hline Low Re. smooth & 324.75 & 277.46 & $14.56 \%$ \\
\hline Low Re. skew & 349.06 & 335.16 & $3.98 \%$ \\
\hline High Re. smooth & 1054.97 & 878.64 & $16.71 \%$ \\
\hline High Re. skew & 1615.16 & 1540.95 & $4.59 \%$ \\
\hline High Ro smooth & 584.35 & 522.57 & $10.57 \%$ \\
\hline High Ro skewed & 660.14 & 631.35 & $4.36 \%$ \\
\hline Low Temp. Smooth & 246.91 & 271.11 & $9.80 \%$ \\
\hline Low Temp. Skewed & 314.86 & 310.75 & $1.30 \%$ \\
\hline High Temp. smooth & 1165.16 & 1136.57 & $2.45 \%$ \\
\hline High Temp. skewed & 1359.45 & 1265.73 & $6.89 \%$ \\
\hline
\end{tabular}

Results from only the first 2 passes are used to maintain consistency because, in some cases, experimental data is not available after the root bend.

A trend observed is that the CFD validates bulk heat transfer more accurately against the experiment for turbulated passage geometries. Turbulators break up large secondary flows and 
tend to stabilize a flow by restarting the boundary layer. In a smooth passage, complex flow features can develop gradually along a passage section. Errors that do develop are allowed to propagate and snowball more freely in a smooth passage. Future sections will investigate plate by plate heat transfer correlation, providing greater insight.

For turbulated passages, the variation in net heat transfer rarely exceeds $5 \%$, which is on the order of experimental uncertainty, quoted as $2 \%$ to $7 \%$. The consistent match across the entire operating regime indicates that The CFD model captures the impact of changes in each of the parameters studied. The percent change in heat transfer observed due to variations in Reynolds number, Rotation number, and Buoyancy parameter is consistent between the CFD and experiment for smooth and turbulated geometries. The sensitivity of the experimental results and computational model to each of the perturbations from the baseline rotating condition is documented in Table 12. Baseline is defined as case 4 for smooth walled simulations and case 6 for cases with skewed turbulators.

Table 12. Sensitivity of experiment and CFD to changes in operating condition

\begin{tabular}{|c|c|c|}
\hline & $\begin{array}{c}\text { \%change from baseline } \\
\text { (experiment to experiment) }\end{array}$ & $\begin{array}{c}\text { \%change from baseline } \\
\text { (CFD to CFD) }\end{array}$ \\
\hline Low Re. smooth & $-35.4 \%$ & $-41.6 \%$ \\
\hline Low Re. skew & $-42.4 \%$ & $-45.0 \%$ \\
\hline High Re. smooth & $109.8 \%$ & $85.0 \%$ \\
\hline High Re. skew & $166.4 \%$ & $152.6 \%$ \\
\hline High Ro smooth & $16.2 \%$ & $10.0 \%$ \\
\hline High Ro skewed & $8.9 \%$ & $3.5 \%$ \\
\hline Low Temp. Smooth & $-50.5 \%$ & $-42.9 \%$ \\
\hline Low Temp. Skewed & $-48.0 \%$ & $-49.0 \%$ \\
\hline High Temp. smooth & $131.7 \%$ & $139.4 \%$ \\
\hline High Temp. skewed & $124.2 \%$ & $107.5 \%$ \\
\hline
\end{tabular}




\subsubsection{Detailed Validation}

With the general impression that the computational model captures bulk heat transfer and the impacts of the various physics at play in the HOST passage, more localized validation is performed. Heat transfer data from the HOST program is available for each of the 4 passage walls at 16 stream wise "plate" locations. CFD results are post-processed to match the data extraction methodology at these same locations. For each operating point considered, local heat transfer is compared against experiment, and conclusions are drawn about the passage physics and model capabilities.

\subsubsection{Stationary Baseline Condition}

The stationary baseline condition benchmarks the computational model against experiment with as few physics involved as possible. In the absence of rotation, Coriolis Effect does not exist, and the impact of Buoyancy is vastly reduced. The stationary condition is primarily characterized by entrance effect and bend behavior. Figure 36 compares the net heat transfer at various stations along the passage between CFD and experiment for the smooth walled passage geometry, corresponding to Case 1 . 


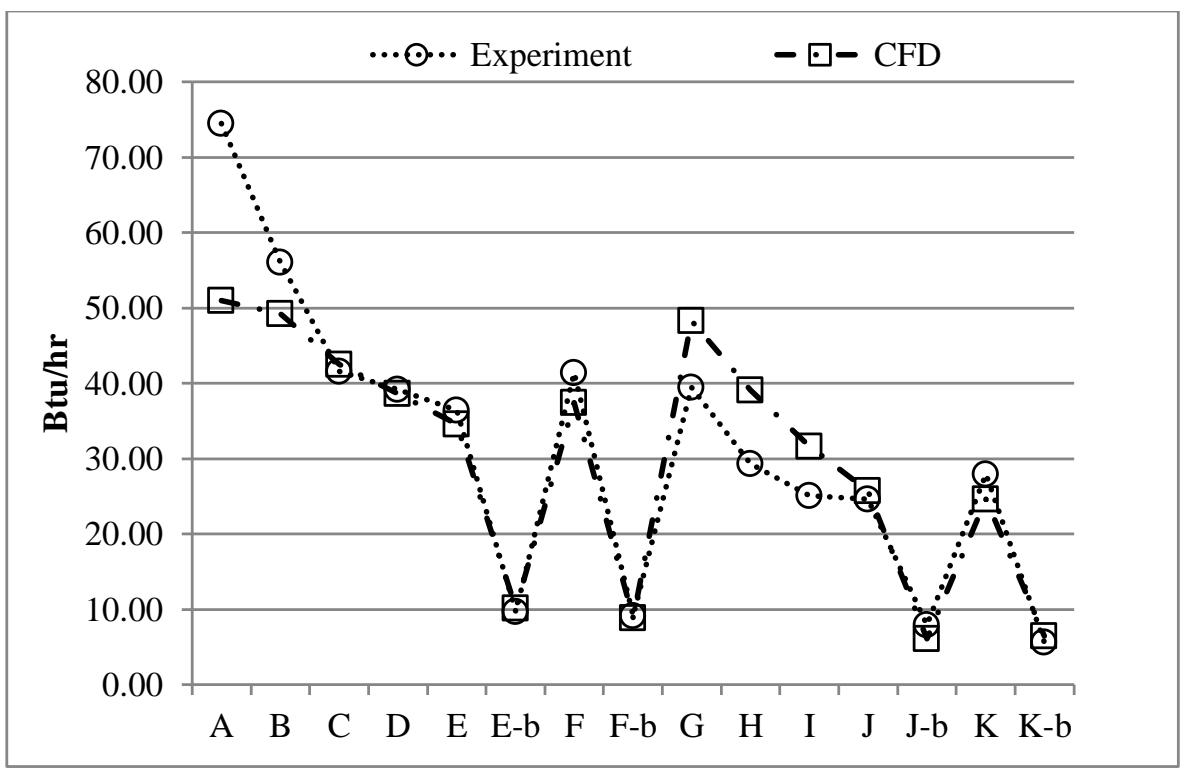

Figure 36. Heat transfer along passage for Case 1

The heat transfer shown includes all heated plates from the experiment, including leading, trailing, and side walls. The first 2 stations exhibit a significant underprediction compared to experimental results. In spite of applying HOST program measurements for inlet velocity profile and turbulence intensity, entrance effects appear underpredicted by the CFD model. The behavior of the plenum setup in the HOST experimentation is not reflected properly using the applied CFD inlet condition. As discussed, specifics of the plenum and inlet setup from the HOST tests are not immediately available. Good data agreement is achieved downstream of entrance effects through the end of bend 1 (stations $\mathrm{E}$ and F). In the inflowing leg, the CFD results indicate higher heat transfer than observed in HOST testing. As the flow enters the root bend (stations $\mathrm{J}$ and $\mathrm{K}$ ) the heat transfer rate again matches well between CFD and experiment.

The third leg is not included in net heat transfer validation because the data was not provided in the HOST documentation beyond the root turn at some test conditions. Error analysis indicates that the experimental error due to accumulated error in the energy balance could reach $30 \%$ in the final outflowing leg. [2] 
Addition of turbulators to the stationary baseline operating point adds complexity to the flowfield. Turbulators along the leading and trailing walls reset the boundary layer and enhance turbulent mixing. This mixing also creates turbulent structures which tend to suppress the impact of other secondary flows. The total heat transfer along the passage with straight turbulators, orthogonal to the flow, is plotted in Figure 37.

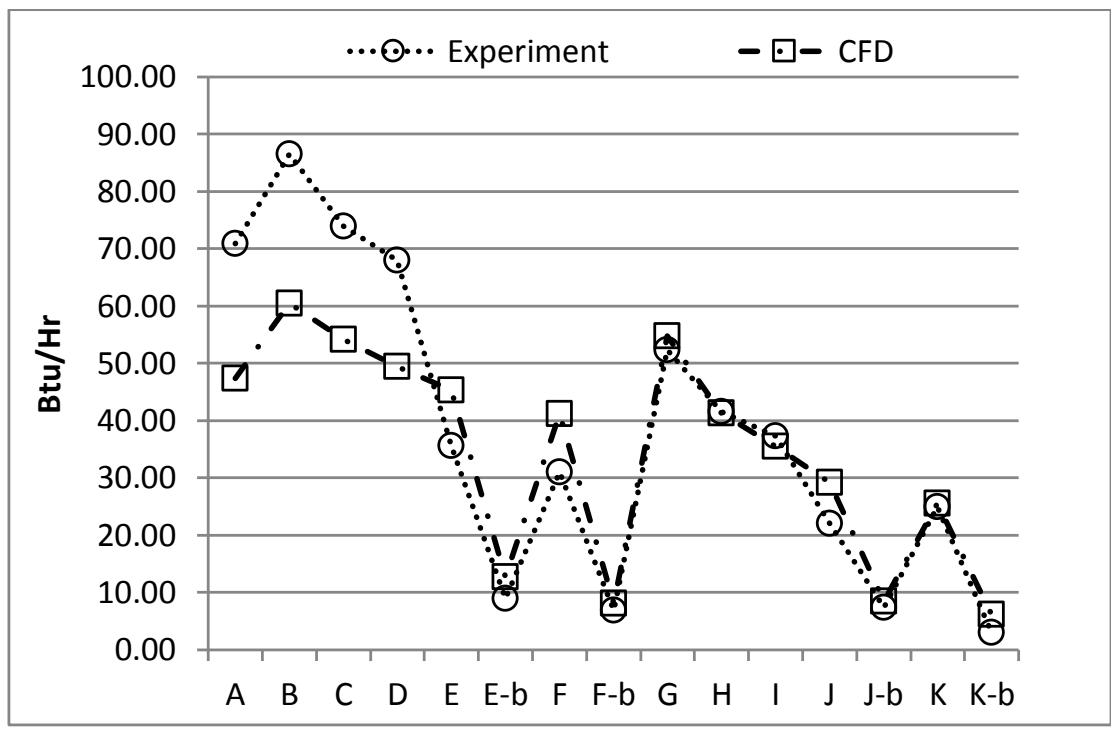

Figure 37. Heat transfer along passage for Case 2

Under-prediction of heat transfer in the first leg due to inlet condition appears once again.

Downstream of bend 1, excellent agreement is achieved.

Net heat transfer in Case 3 is illustrated in Figure 38 with turbulators skewed 45 degrees relative to the passage orientation 


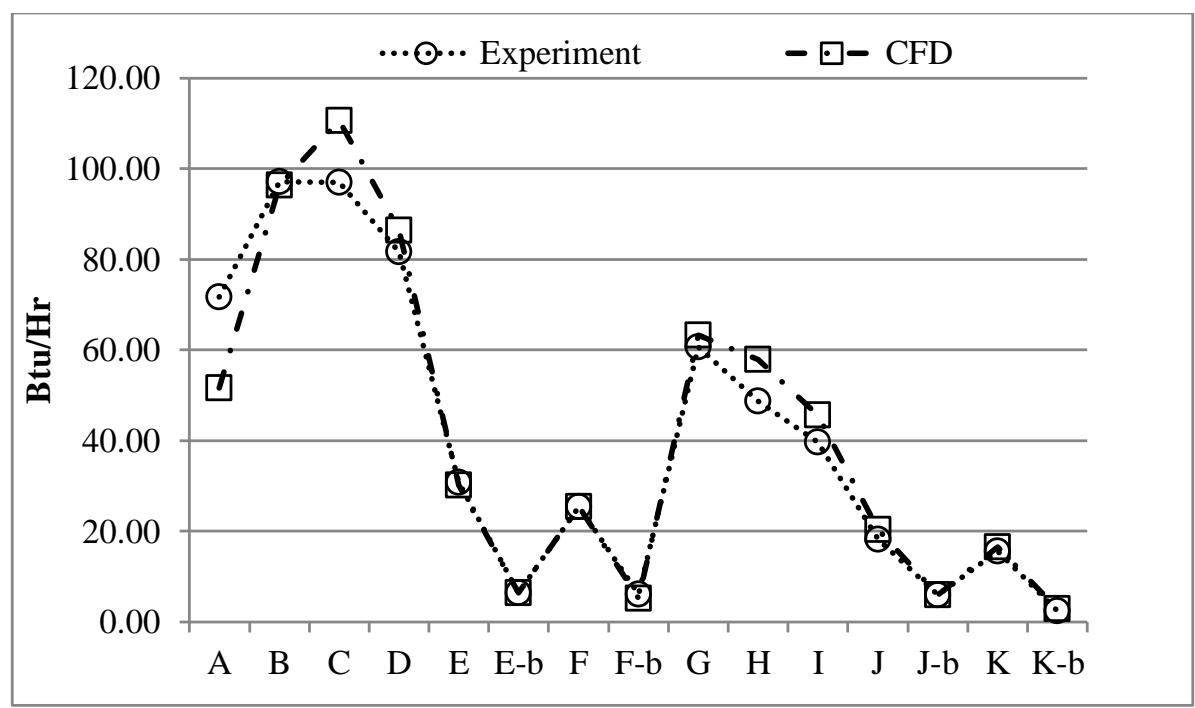

Figure 38. Heat transfer along passage for Case 3

The presence of turbulators enhances mixing near the wall, increasing the net heat transfer significantly. Similar to the smooth operating point, the CFD model underpredicts entry effects and overpredicts heat transfer in the inflowing leg. The skewed turbulators develop a secondary flow which appears to desensitize the first leg heat transfer to the passage inlet condition. Disparaties from experimental results are less noticable with skewed turbulators present. The impact of the turbulators appears well captured by the computational model.

Plate by plate heat transfer represents the highest resolution at which experimental data from the HOST program is available. CFD results were postprocessed to allow back to back comparison of the heat transfer through each of the plates on individual walls for comparison. Figure 39 illustrates this comparison for the Stationary Baseline operating condition with no turbulators, "straight " turbulators, and skewed turbulators. Heat transfer is represented in terms of Nusselt Number. 


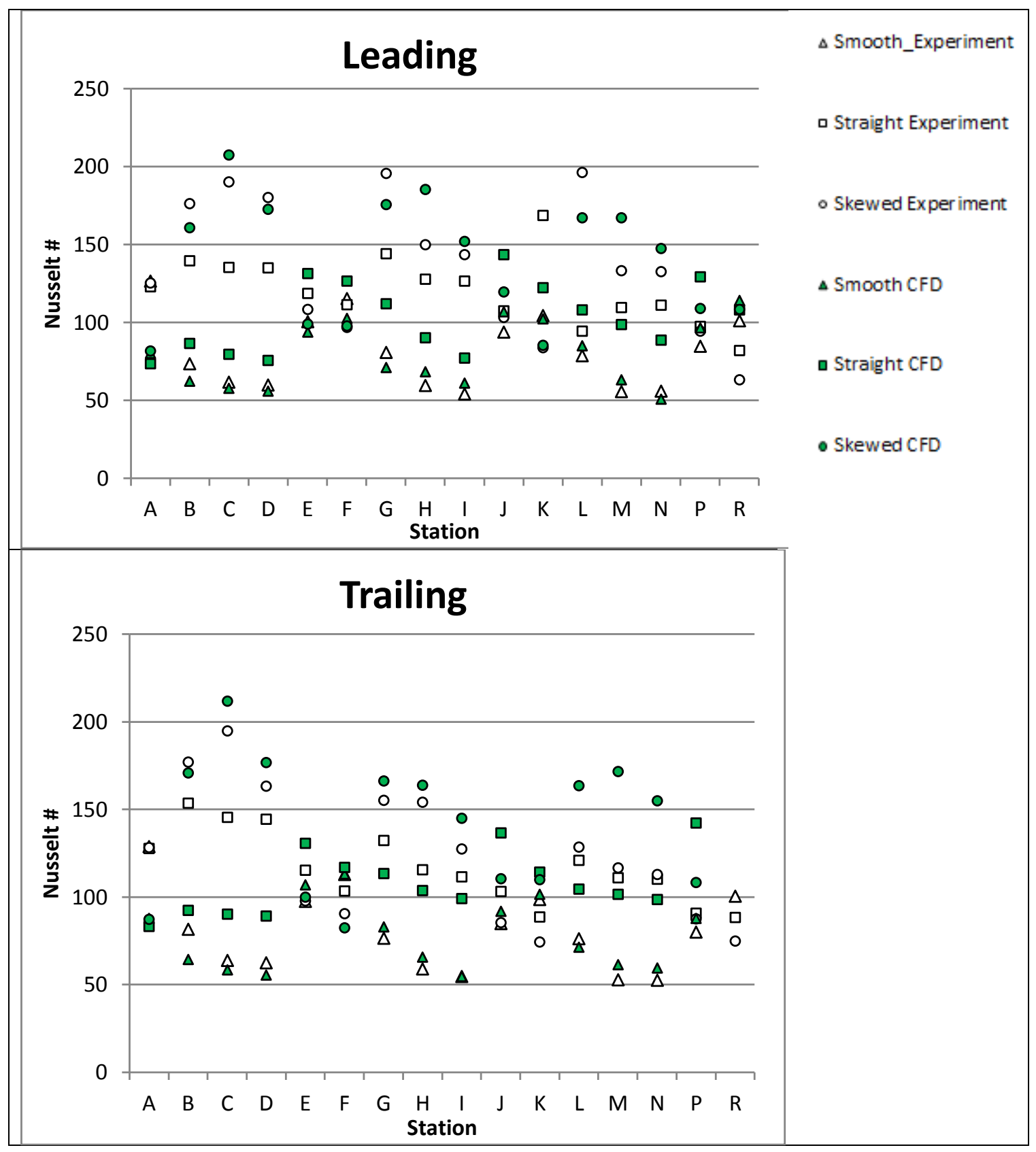




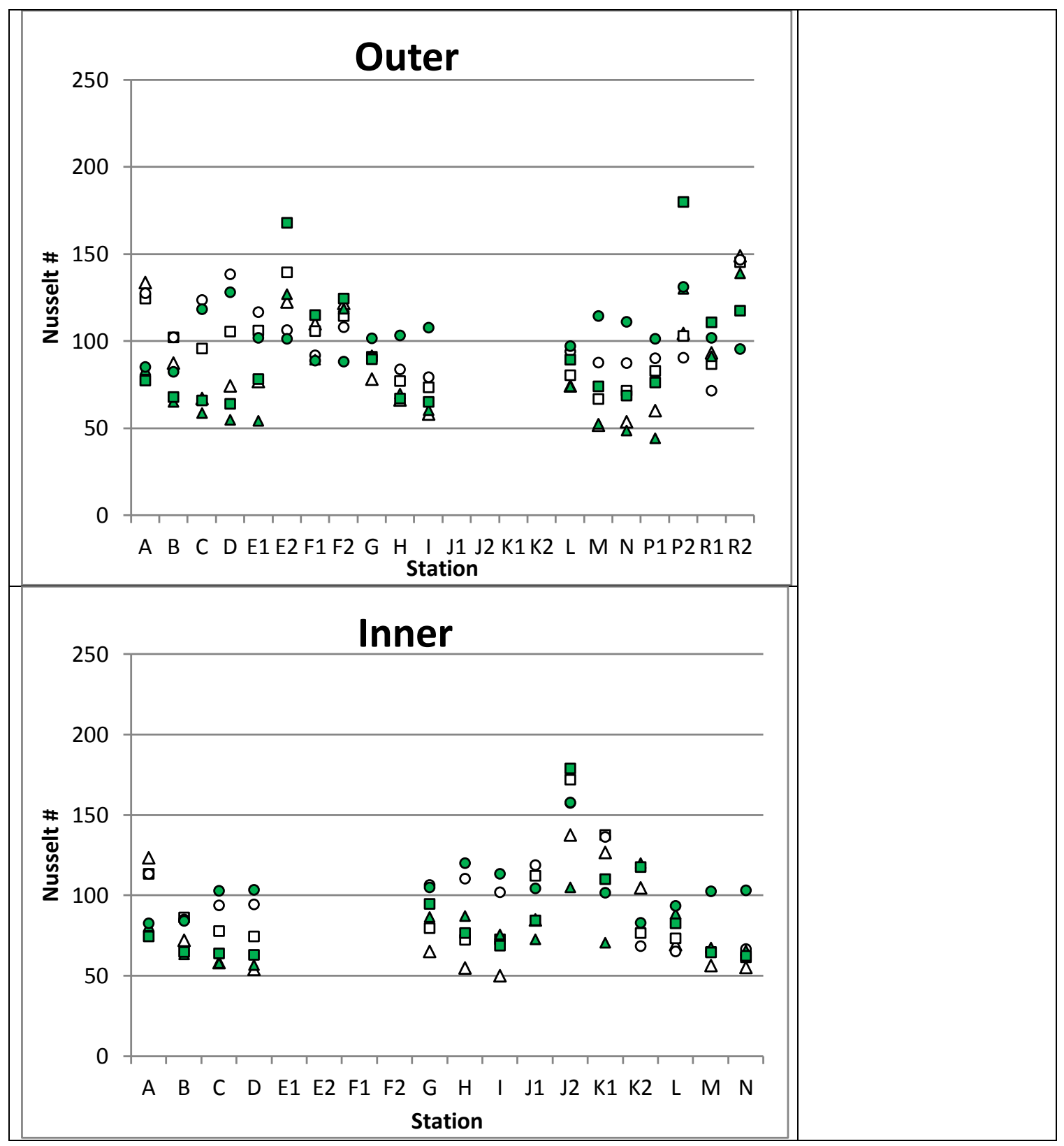

Figure 39. Passage Nusselt number for stationary operation

Eddies are generated in the passage due to bend effects, turbulators, and other phenomena which interact to create a complex flow. Individual plate heat transfer data is more sensitive to these secondary turbulent structures than station by station results, such as the results shown in Figure 36, Figure 37, and Figure 38. Station by station heat transfer, including leading, trailing, and side walls as one heat transfer surface, provides a measure of net heat transfer augmentation along the duct due to the various physical phenomena. Agreement between CFD and experimental data on 
a station by station basis indicates that the magnitude of mixing and turbulence generation at any streamwise location is represented properly. Figure 39, which illustrates heat transfer on a plate by plate basis, is sensitive to the exact location at which turbulent flow structures interact with sidewalls. Agreement between CFD and experimental data on a plate by plate basis indicates a capture of the passage physics down to the location at which turbulent structures and eddies that are generated interact with individual walls. With RANS turbulence modelling and modern CFD capability, such agreement is challenging to achieve.

Away from the entrance, the distribution of heat transfer on a plate by plate basis is reasonably well matched for cases 1,2 , and 3 .

Because flow behavior within a turbine cooling passage is complex, it is difficult to evaluate the agreement to CFD results by comparing heat transfer magnitude directly between CFD results and Experimental results. For example, if good data match is attained at Case 1, which is smooth walled and stationary, but a poor data match is observed at an operating point which includes reduced flowrate, rotation, and skewed turbulators, it is difficult to determine specific shortcomings of the model without further analysis. In order to evaluate model sensitivity to various physics at play, the change in heat transfer due to a single change in operating point is analyzed.

In order to evaluate the ability of the CFD model to capture the impact of leading and trailing wall turbulators, The change in heat transfer predicted by the CFD model between smooth and turbulated results is compared to the change in heat transfer measured in the HOST data set. $\% \Delta$ $\mathrm{Nu}$ going from case $\mathrm{A}$ to case $\mathrm{B}$ is defined as:

$$
\frac{N u_{A}-N u_{B}}{N u_{B}}
$$


The impact of each turbulator type, straight and skewed, is compared against the smooth walled geometry for the stationary operating point in Figure 40.

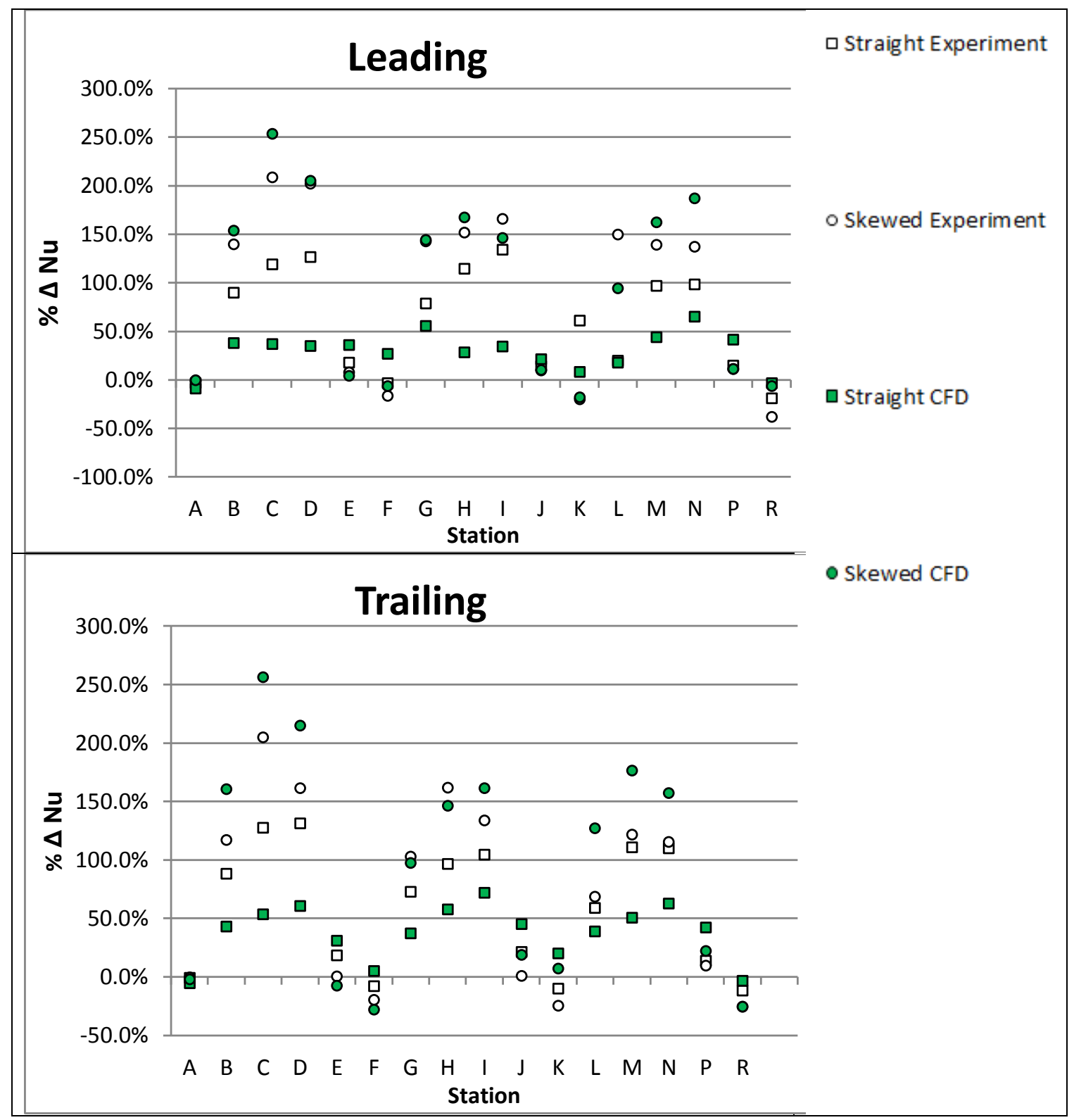




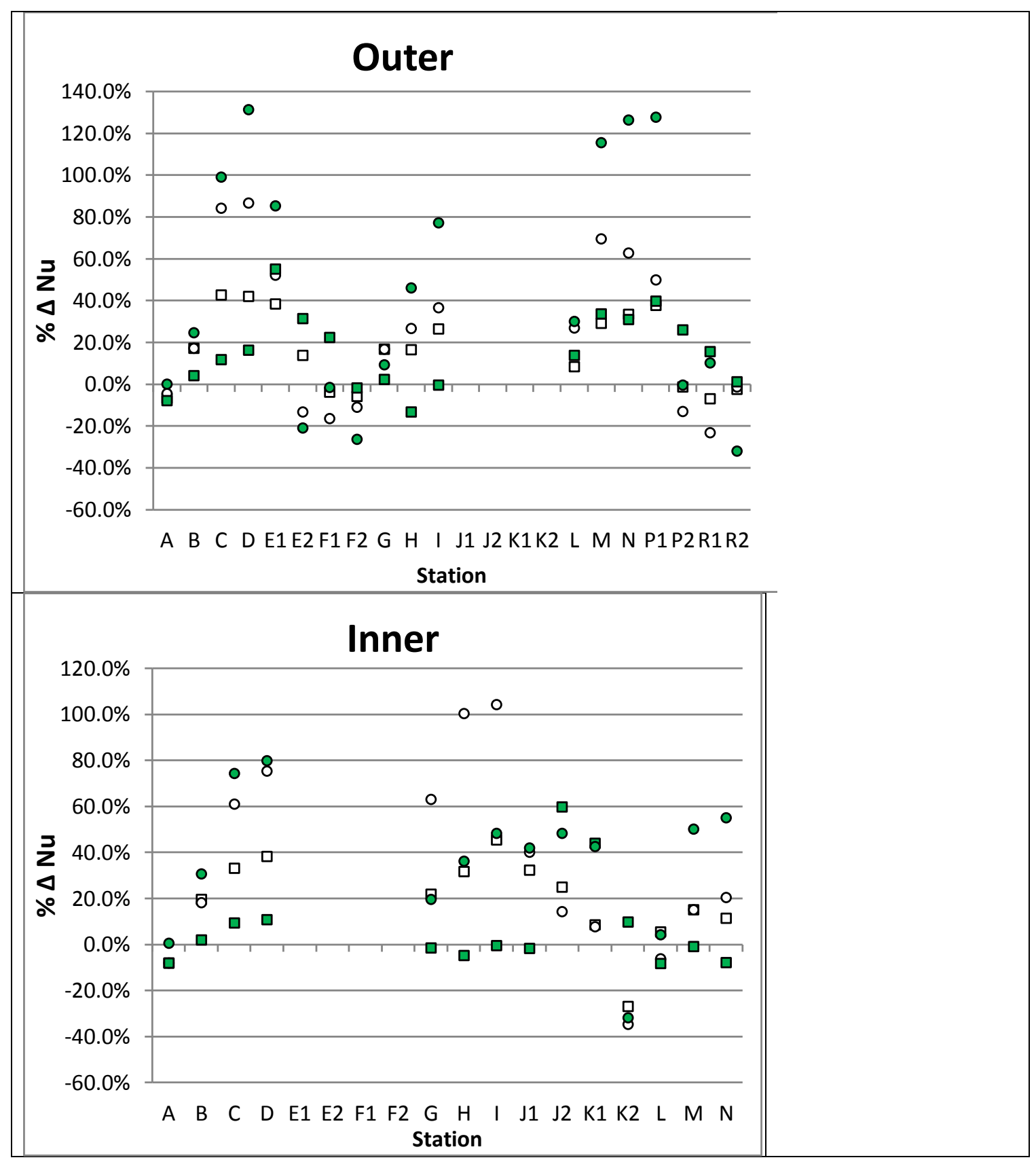

Figure 40. Impact of various turbulator geometries on passage heat transfer 
It appears that the impact of the straight turbulators is relatively poorly represented in the CFD model. Because the primary purpose of this effort is to validate a model used to improve the understanding of flow in passages with skewed turbulators, Investigation into the cause of disagreement between straight turbulated CFD results and experimental data was minimal in order to budget time to higher priority information. A likely cause for the degraded data match with straight turbulators is increased separation and reattachment which is historically difficult to capture with RANS CFD.

With some exceptions late in the passage concerning the outer and inner sidewall heat transfer, the CFD model appears to capture the impact of adding skewed turbulators to the stationary passage well.

\subsubsection{Rotating Baseline Condition}

The rotating baseline condition adds the impact of rotation to the stationary baseline operating point. Reynolds number and density ratio are held constant. The total heat transfer at each station along the passage is plotted in Figure 41 for the smooth walled rotating baseline case. 


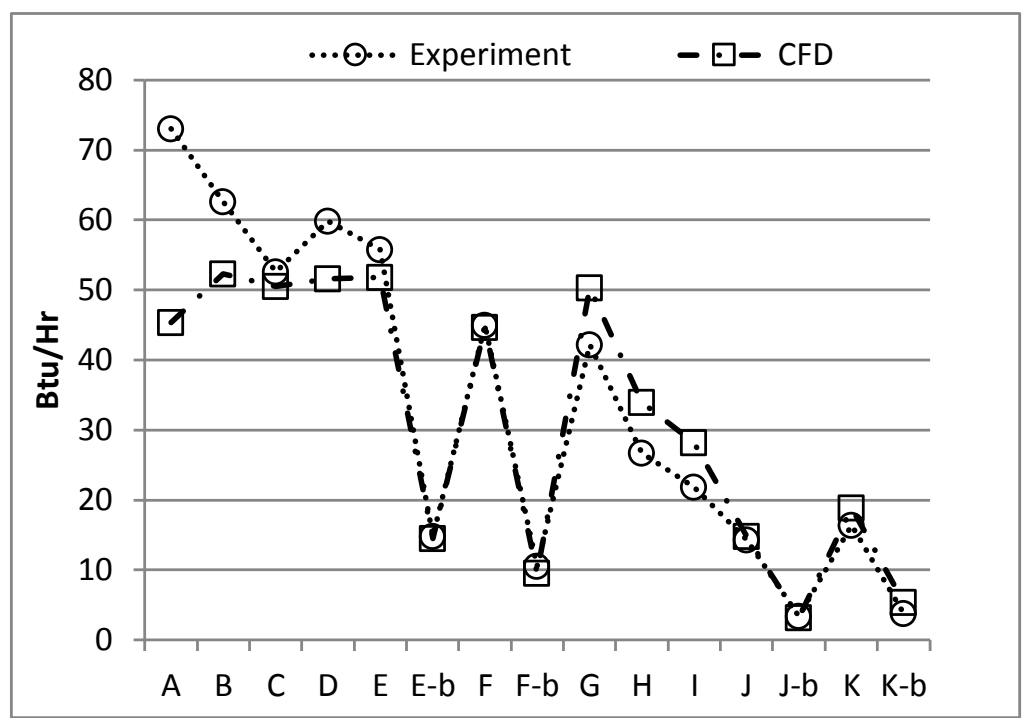

Figure 41. Heat transfer along passage for Case 4

The CFD model differs from experimental results primarily in terms of entrance effect due to inlet condition, and the behavior downstream of the first bend.

Figure 42 similarly plots section heat transfer along the rotating passage for case 5, with straight turbulators.

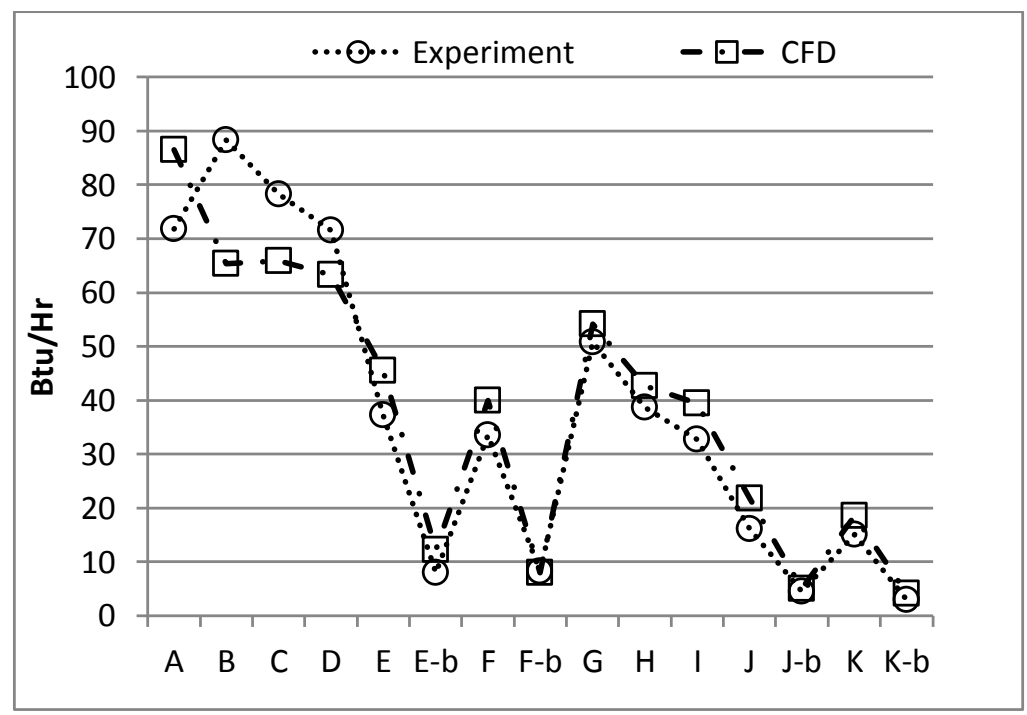

Figure 42. Heat transfer along passage for Case 5 
Downstream of the entrance, the agreement between CFD and experiment in terms of combined heat transfer on all 4 walls is very good. Heat transfer along the rotating passage with skewed turbulators, case 6, is illustrated in Figure 43.

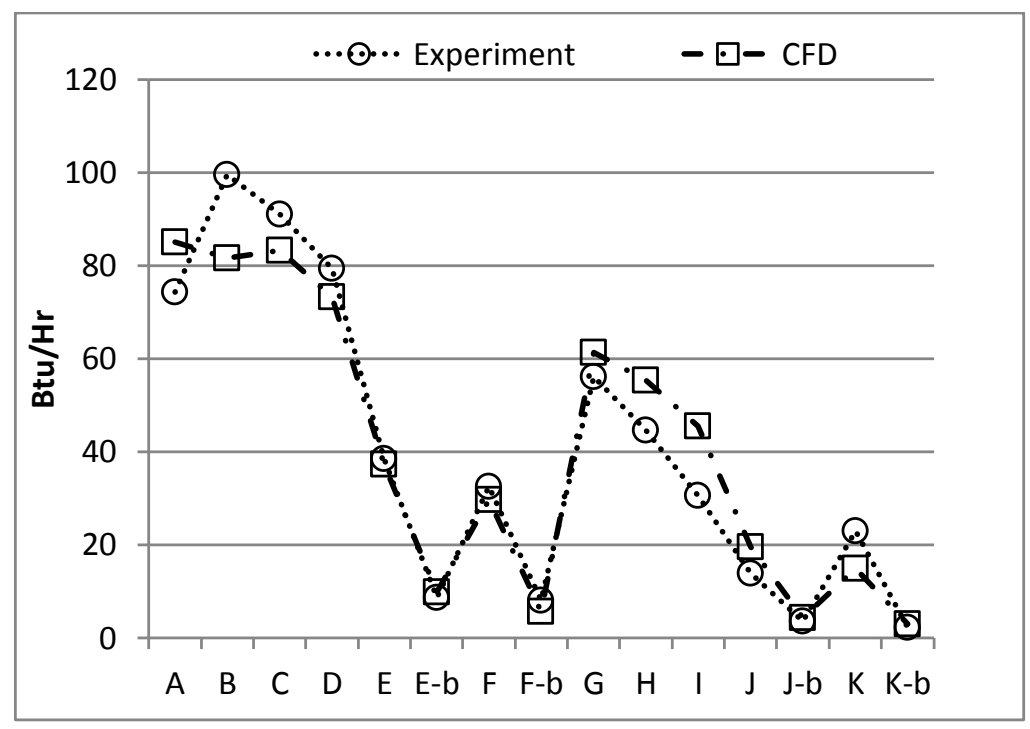

Figure 43. Heat transfer along passage for Case 6

$\mathrm{Nu} / \mathrm{Nu}_{\infty}$ for each of the plates within the passage for the rotating baseline condition, case 4,5 , and 6 is illustrated in Figure 44. 


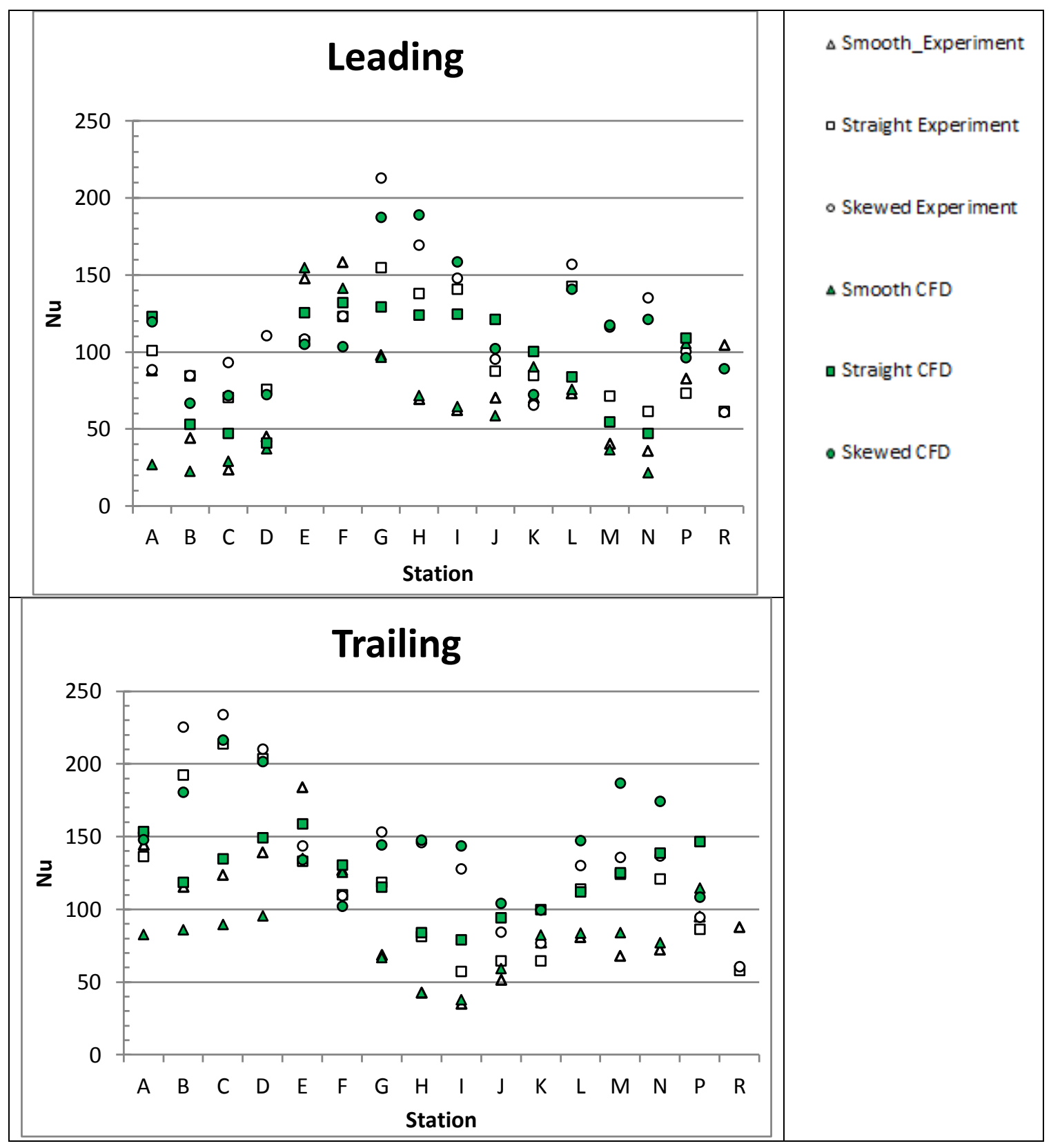




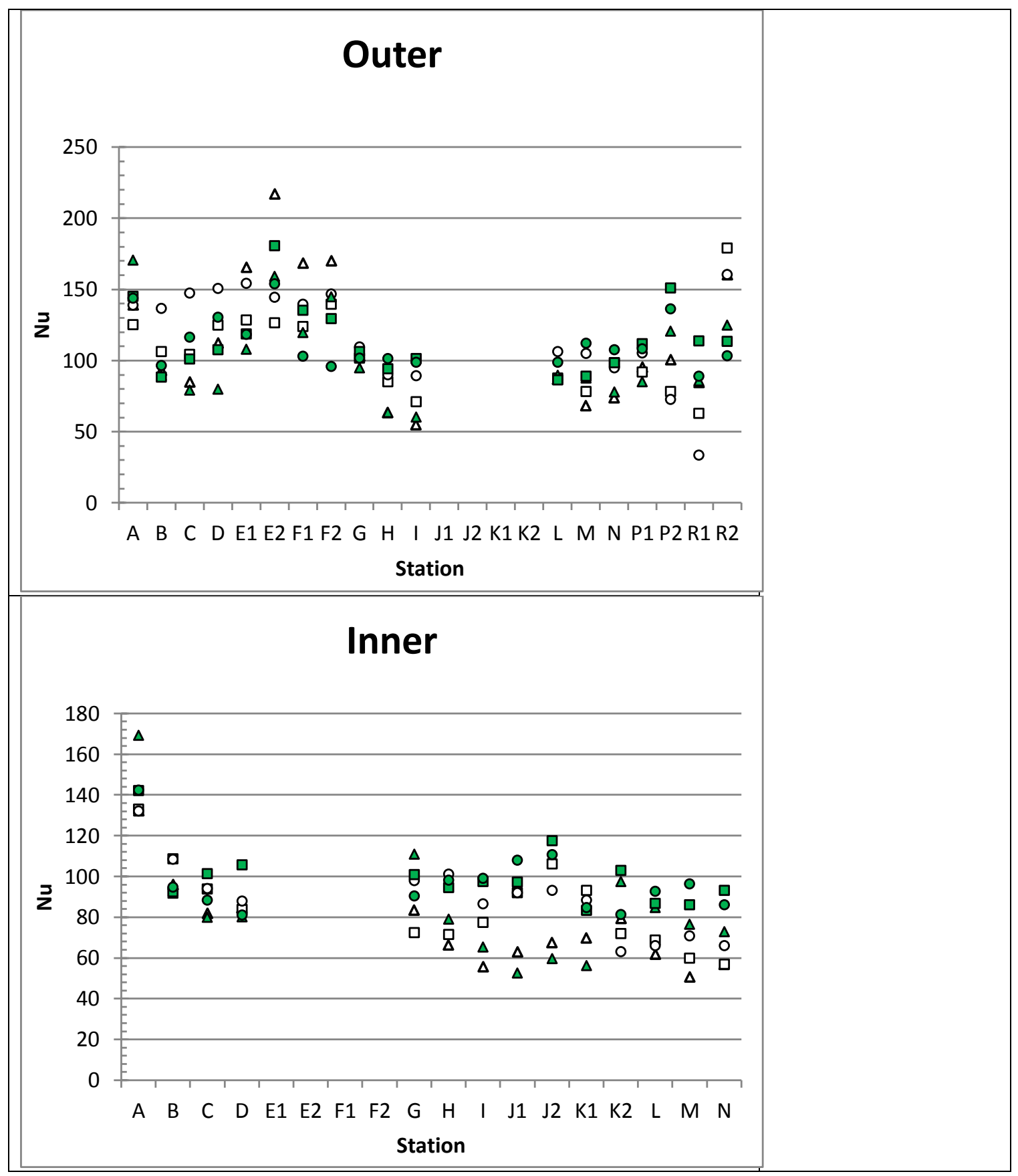

Figure 44. Nusselt number for baseline rotating operating point simulations, Cases 4, 5, and 6

Similar to stationary operation, the straight turbulator geometry results show the greatest deviation from experiment. The behavior in the passage is significantly altered from stationary operation, with the trailing walls showing enhanced heat transfer for outflowing legs and degraded heat transfer on inflowing legs, as expected. The suction side, leading wall demonstrates 
the opposite trend. Overall, a reasonable level of agreement is achieved between CFD and experiment.

In order to isolate the impact of rotation on passage heat transfer, the percent increase in $\mathrm{Nu} / \mathrm{Nu} \infty$ from the stationary operating point to the rotating baseline operating point is plotted in Figure 45 . 


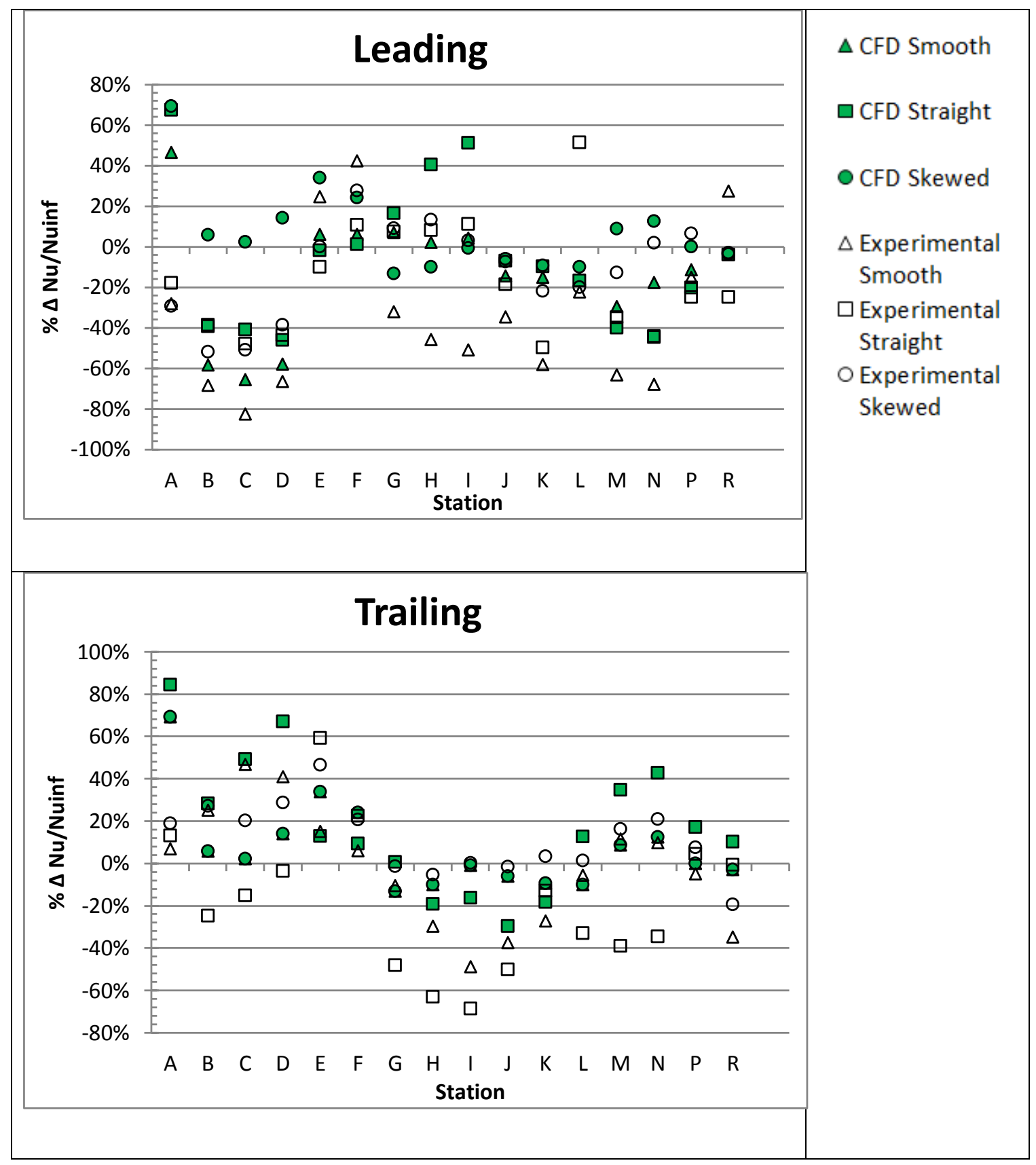




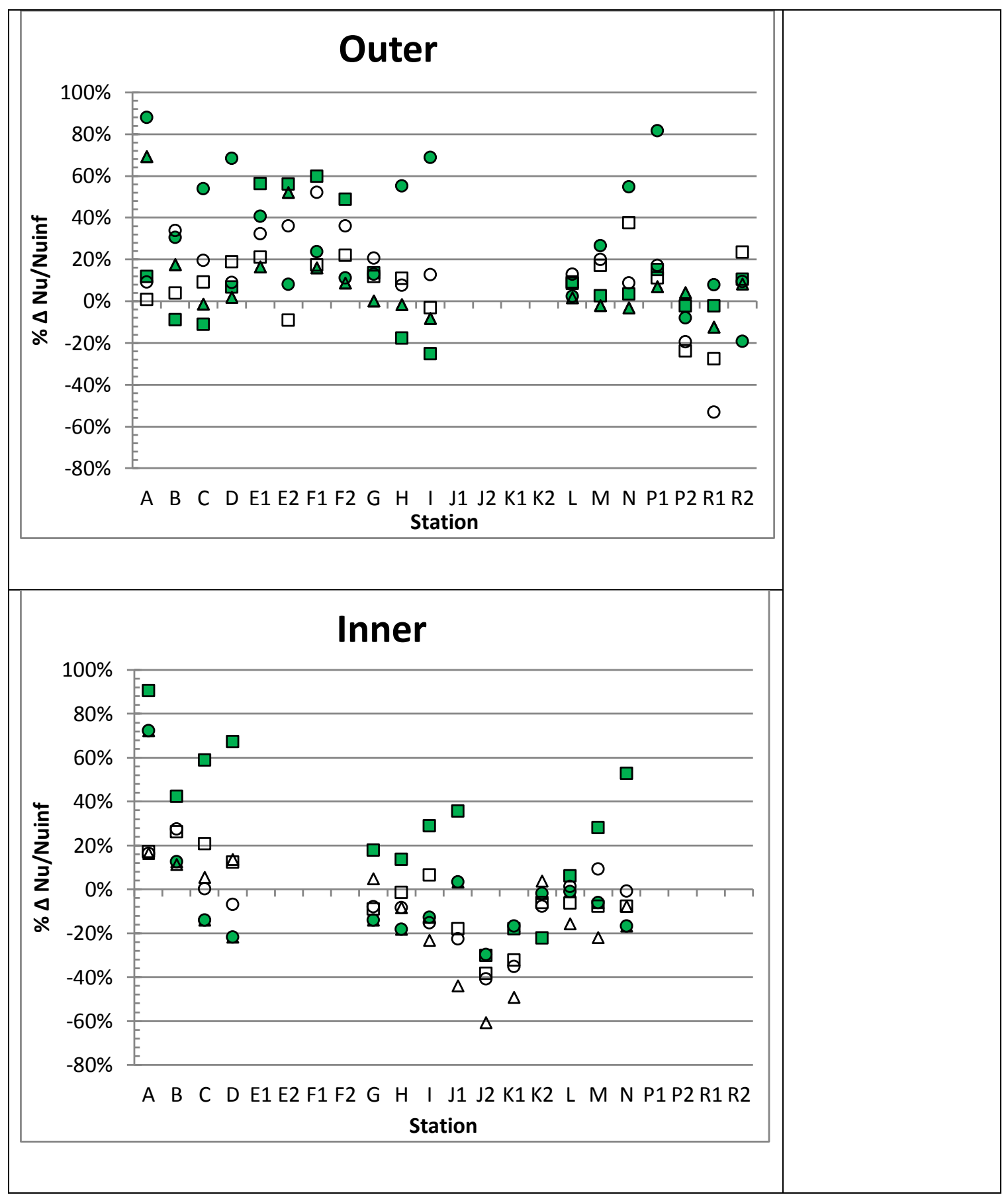

Figure 45. \% variation in Nusselt number associated with rotating vs. stationary baseline cases

The leading, suction wall experiences decreased shear on the first outflowing leg when the passage is rotating. Legs further downstream exhibit less influence of rotation. Case 6, the skewed rotating baseline simulation, differs from the others and from the experiment in this first 
leg. Aside from the leading wall for case 6, the impact of rotation appears well captured by the CFD model.

Cases 1 through 6 are considered as the baseline data sets for HOST validation. The calculated Nusselt numbers are compared to the experimentally measured Nusselt numbers for all plates and each of these operating points in Figure 46. The solid line represents an exact agreement between CFD and experiment. The dashed lines represent $\pm 20 \%$ error.

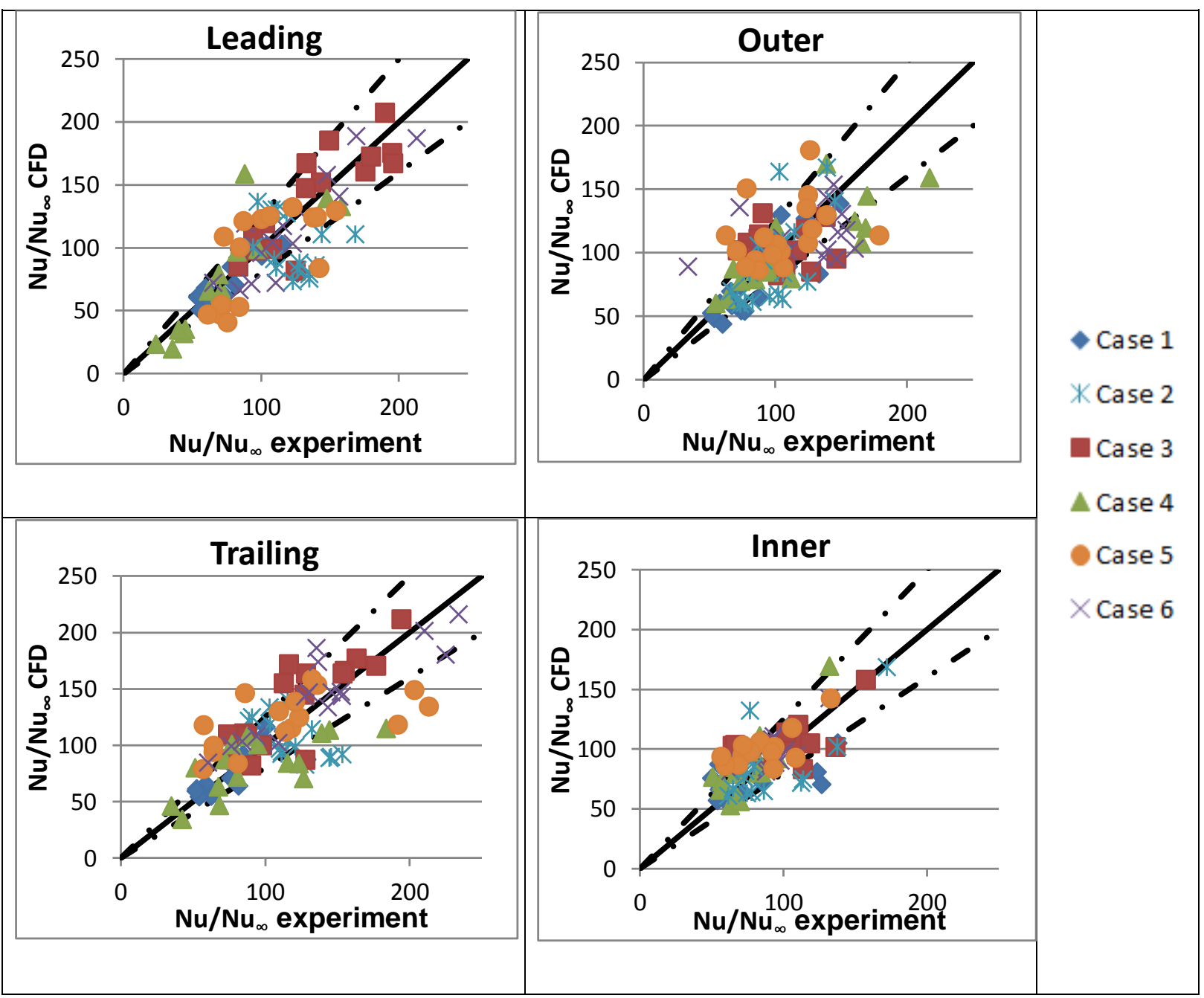

Figure 46. 45 degree plots representing error of baseline set of operating points

Consistent with previous observations, the straight turbulator datasets, case 2 and case 4 , which correspond to straight turbulators, are consistently outside the $20 \%$ error margin. Other datasets 
have outliers which exceed $20 \%$ error, but are generally within the dashed lines representing $20 \%$ error.

Cases 15 through 18 explore the impact of variations to Reynold number relative to the baseline rotating operating condition with smooth walls and skewed turbulators. Reynolds number is reduced from 25,000 at the baseline operating point to 12,500 for cases 15 and 17 , which feature smooth walls and skewed turbulators, respectively. Reynolds number is increased from 25,000 to 50,000 for case 16 with smooth walls and increased to 75,000 for case 18 with skewed turbulators. Low Reynolds passage operation is of particular concern to this research.

Heat transfer for low Reynolds number operation with smooth passage walls, case 15, is illustrated in Figure 47.

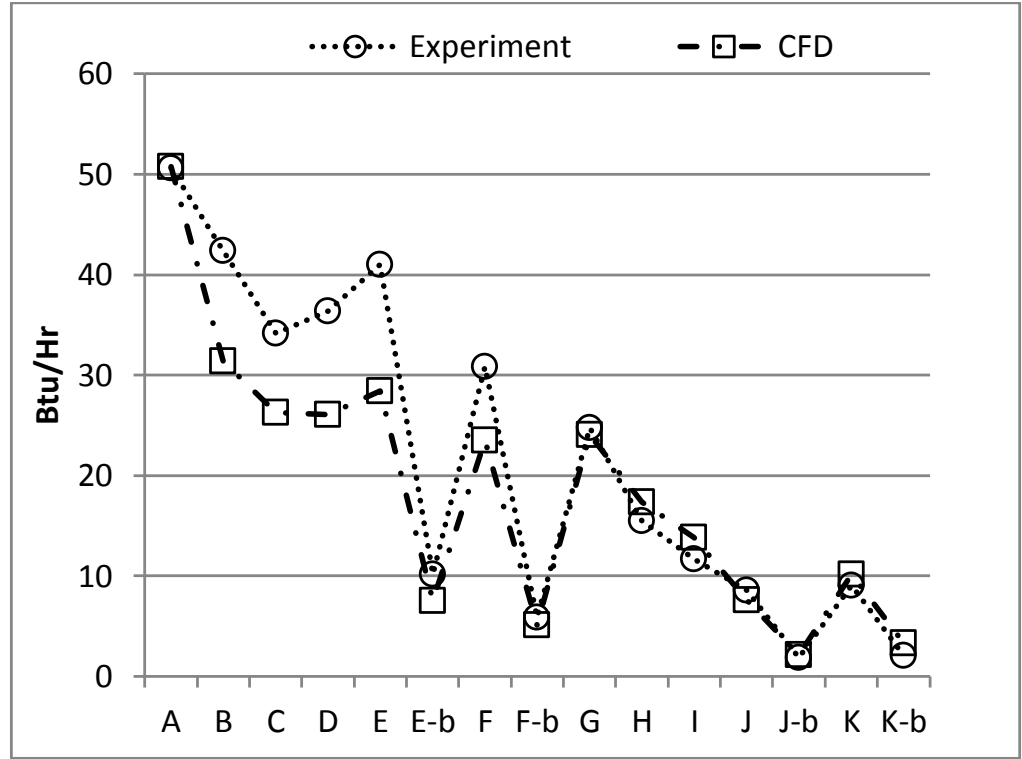

Figure 47. Heat transfer along passage for Case 15

Combined heat transfer of all 4 walls along the passage for case 17, Low Reynolds number with skewed turbulators, appears in Figure 48. 


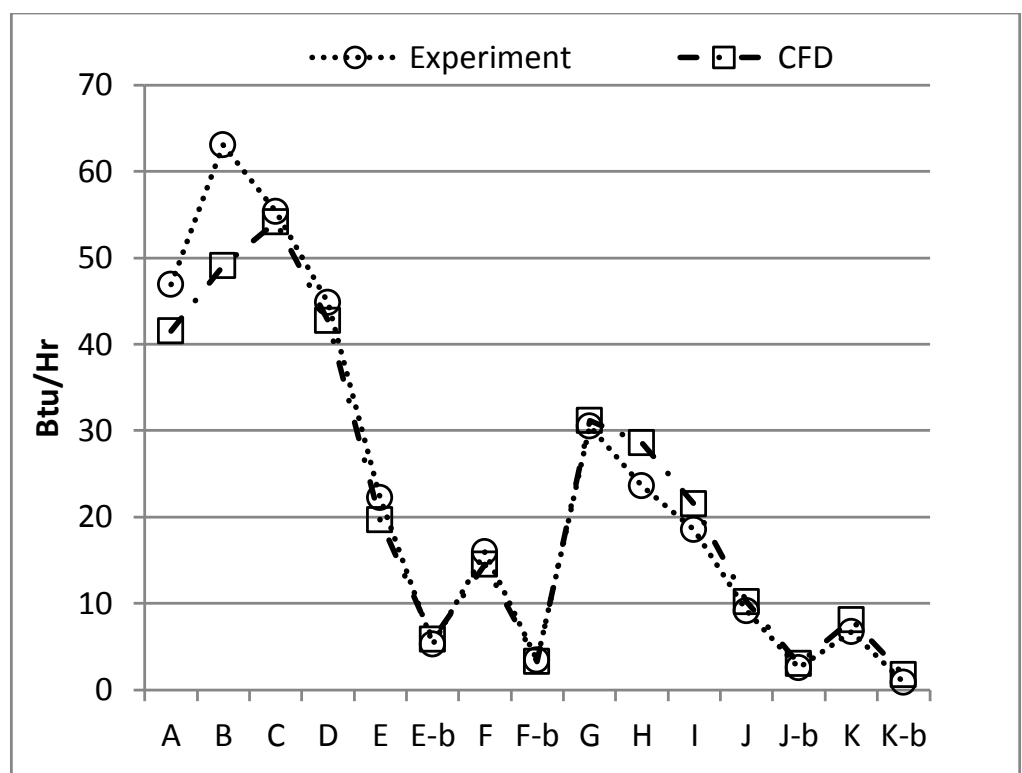

Figure 48. Combined wall heat transfer along passage for Case 17

Heat transfer for low Reynolds number operation with smooth passage walls, case 16, is illustrated in Figure 49. Note that the High Reynolds Number operating point for the smooth walled case, driven by experimental data availability was at $\operatorname{Re}=50,000$. The turbulated high Reynolds Number operating point is at $\operatorname{Re}=75,000$

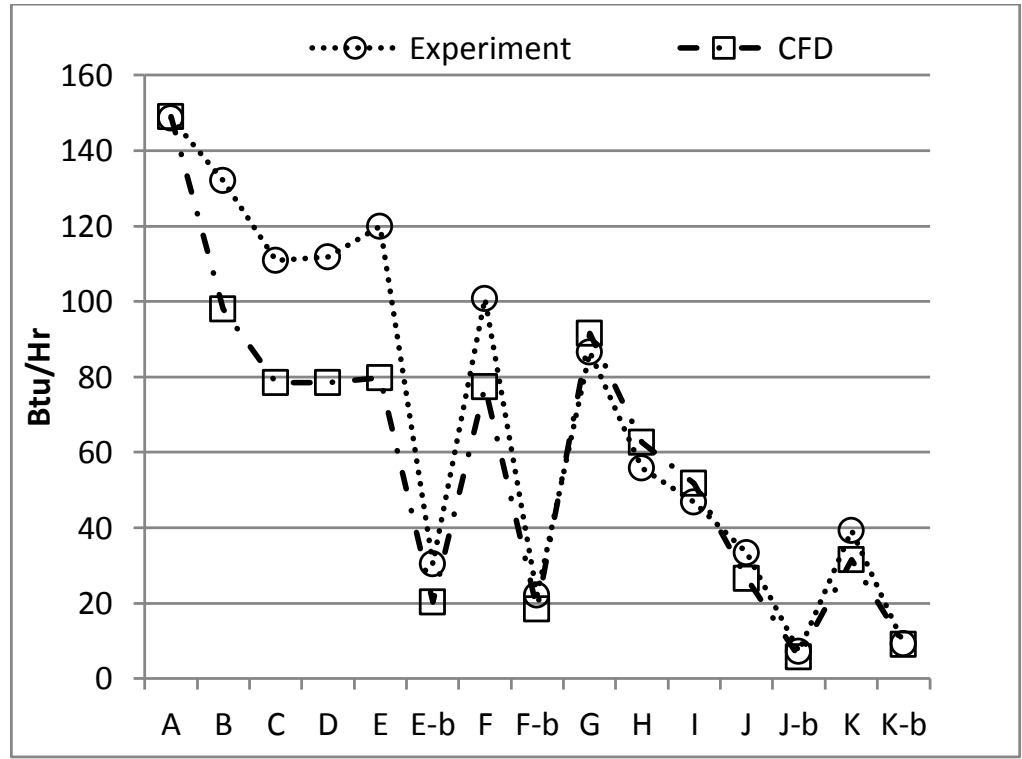

Figure 49. Combined wall heat transfer along passage for Case 16 
Combined heat transfer of all 4 walls along the passage for case 18, high Reynolds number with skewed turbulators, appears in Figure 48.

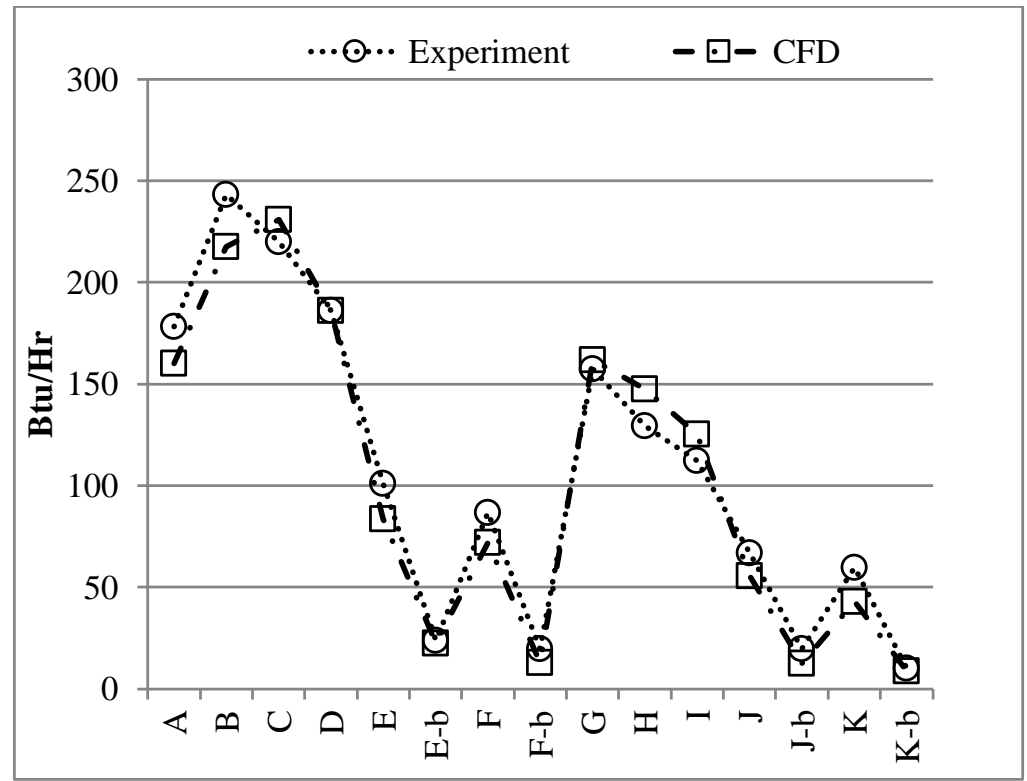

Figure 50. Combined wall heat transfer along passage for Case 18

The impact of Reynolds Number variation for the each heated plate independently is documented in Figure 51. 


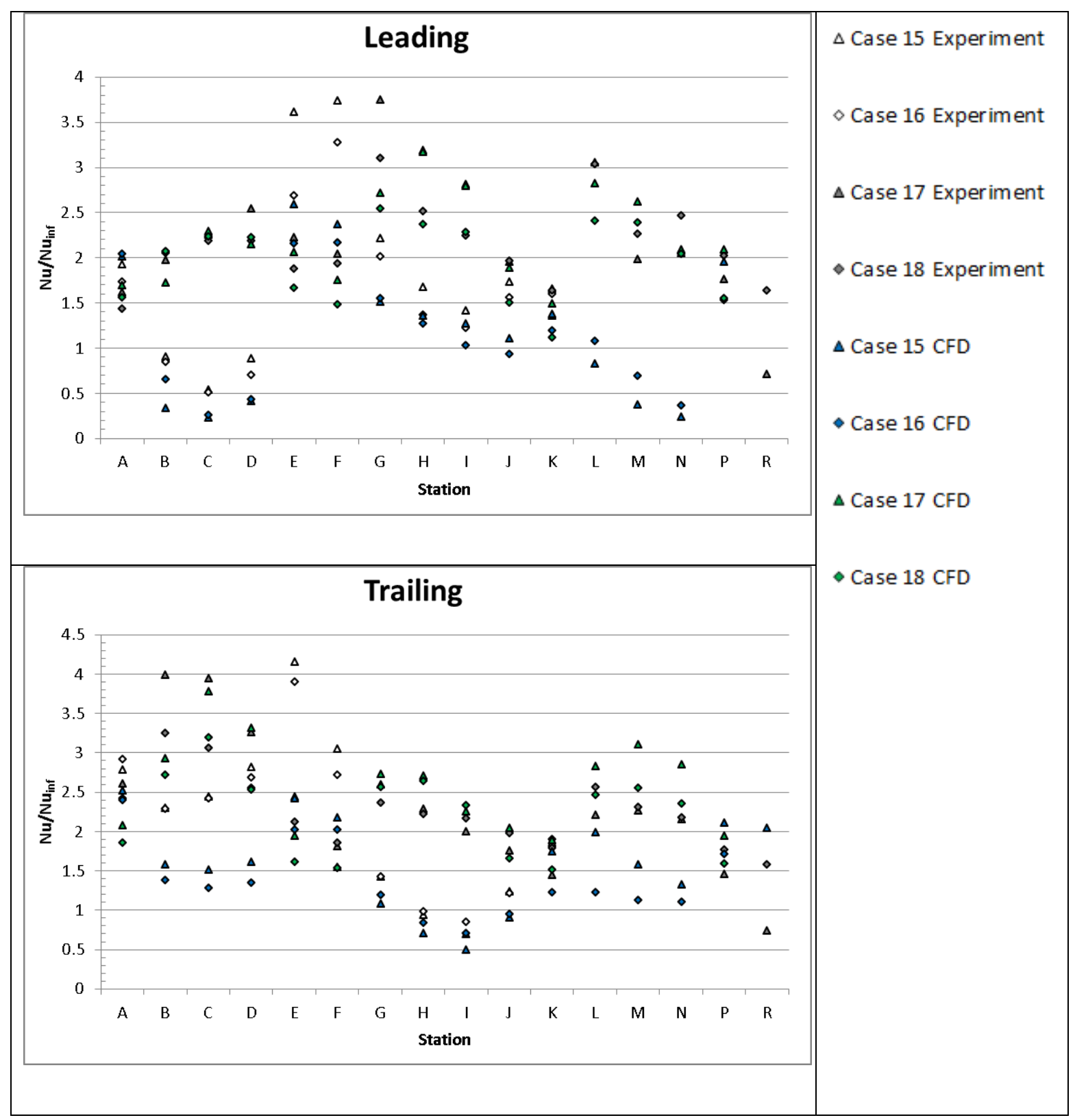




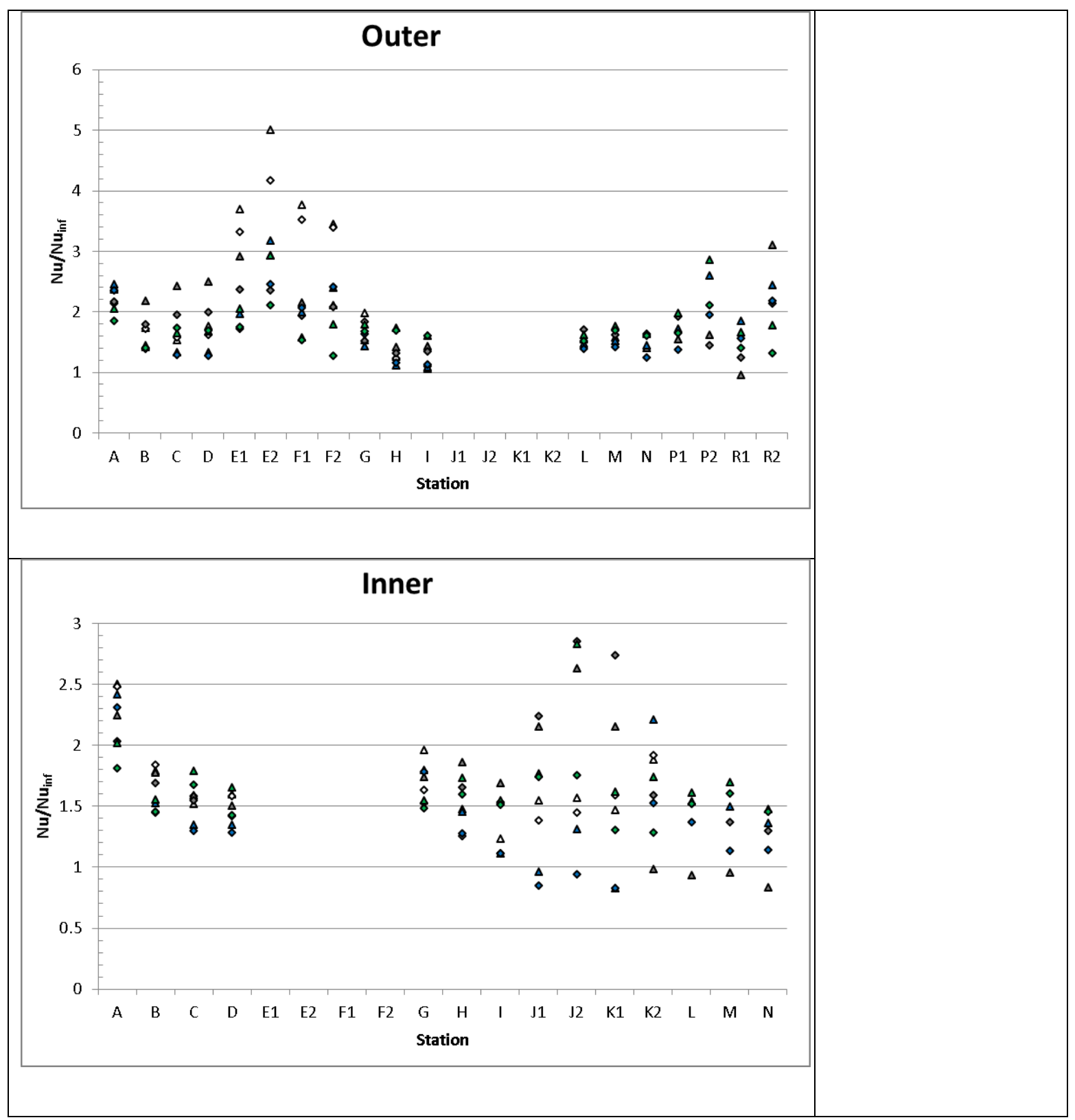

Figure 51. $\mathrm{Nu} / \mathrm{Nu}_{\infty}$ variation throughout passage due to changing Reynolds Number

Impact of Reynolds \# variation is of significant importance to the current research. Behavior has been observed in cooling passages with low passage velocity which do not adhere to correlations developed using the HOST experimental dataset. One purpose of the current effort is to develop a model which can be used to extend the understanding of low Reynolds internal cooling.

There is a strong dependence between Reynolds number and Nusselt number, increasing heat transfer with increasing Reynolds number. When Nusselt number is normalized as $\mathrm{Nu} / \mathrm{Nu}_{\infty}$, The 
impact of increased Reynolds number is fairly small. $\mathrm{Nu} / \mathrm{Nu}_{\infty}$ can be interpreted as the ratio of heat transfer observed to the heat transfer that would occur in a smooth duct with the same bulk velocity. Any impact of Reynolds number observed on this basis is not simply an increase in heat transfer that comes with increased bulk velocity.

Overall, the HOST documentation cites a small impact on Nusselt number ratio due to Reynolds number variation [2]. The HOST data indicates that the greatest sensitivity to Reynolds number exists in the first bend on the leading and outer walls. Figure 52 below shows the change in Nusselt number ratio between the low Reynolds and High Reynolds datasets for turbulated and smooth geometries. Plotting $\Delta N u / N u_{\infty}$ compares the impact of changing Reynolds number only, filtering out any differences between the baseline CFD and experimental results. For the Smooth walled data, the data shown is the increase in $N u / N u_{\infty}$ due to increasing Reynolds number from 15,000 to 50,000 . For the turbulated results the shown data represents the increase in $N u / N u_{\infty}$ due to increasing Reynolds number from 15,000 to 75,000 . 


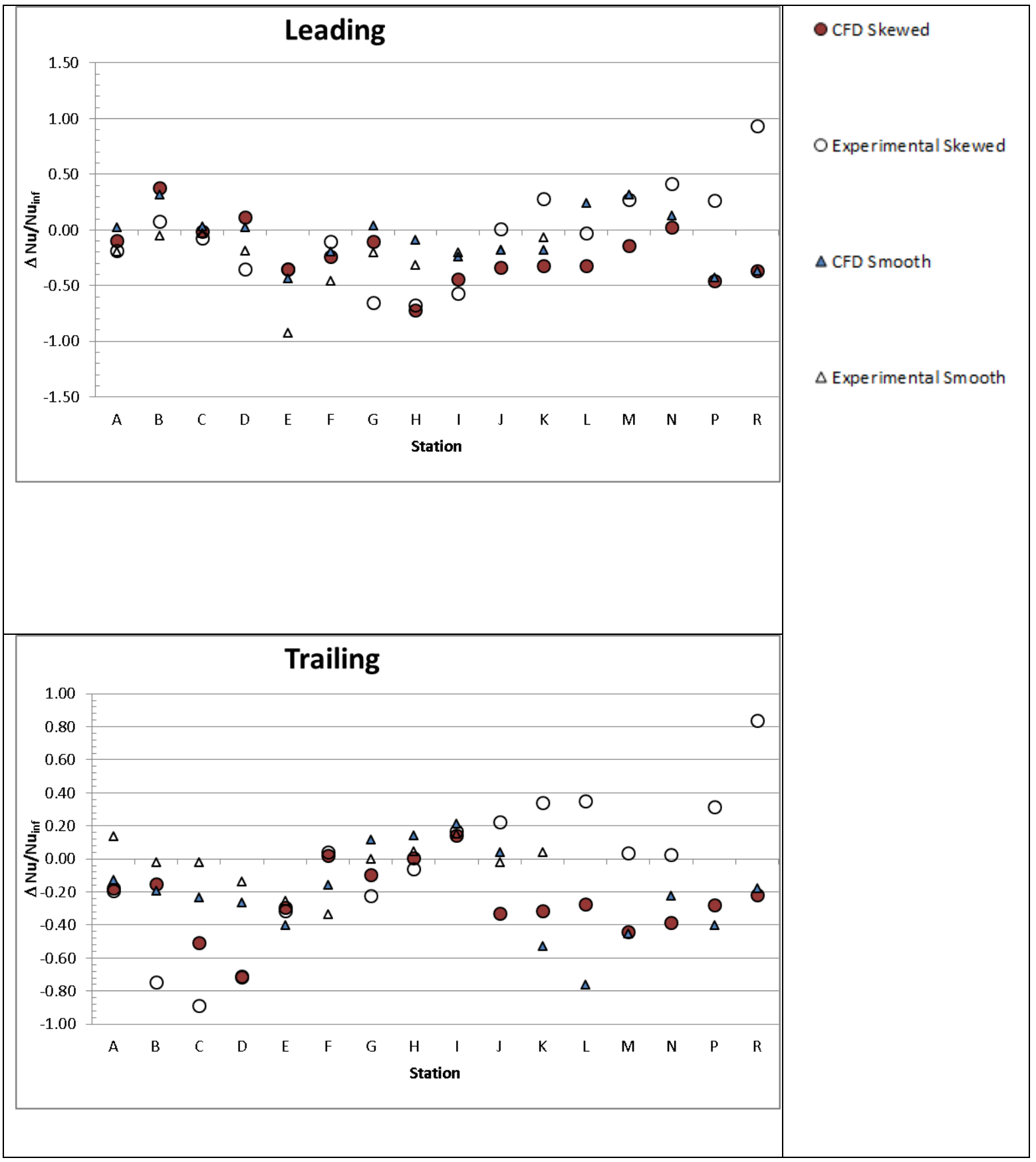




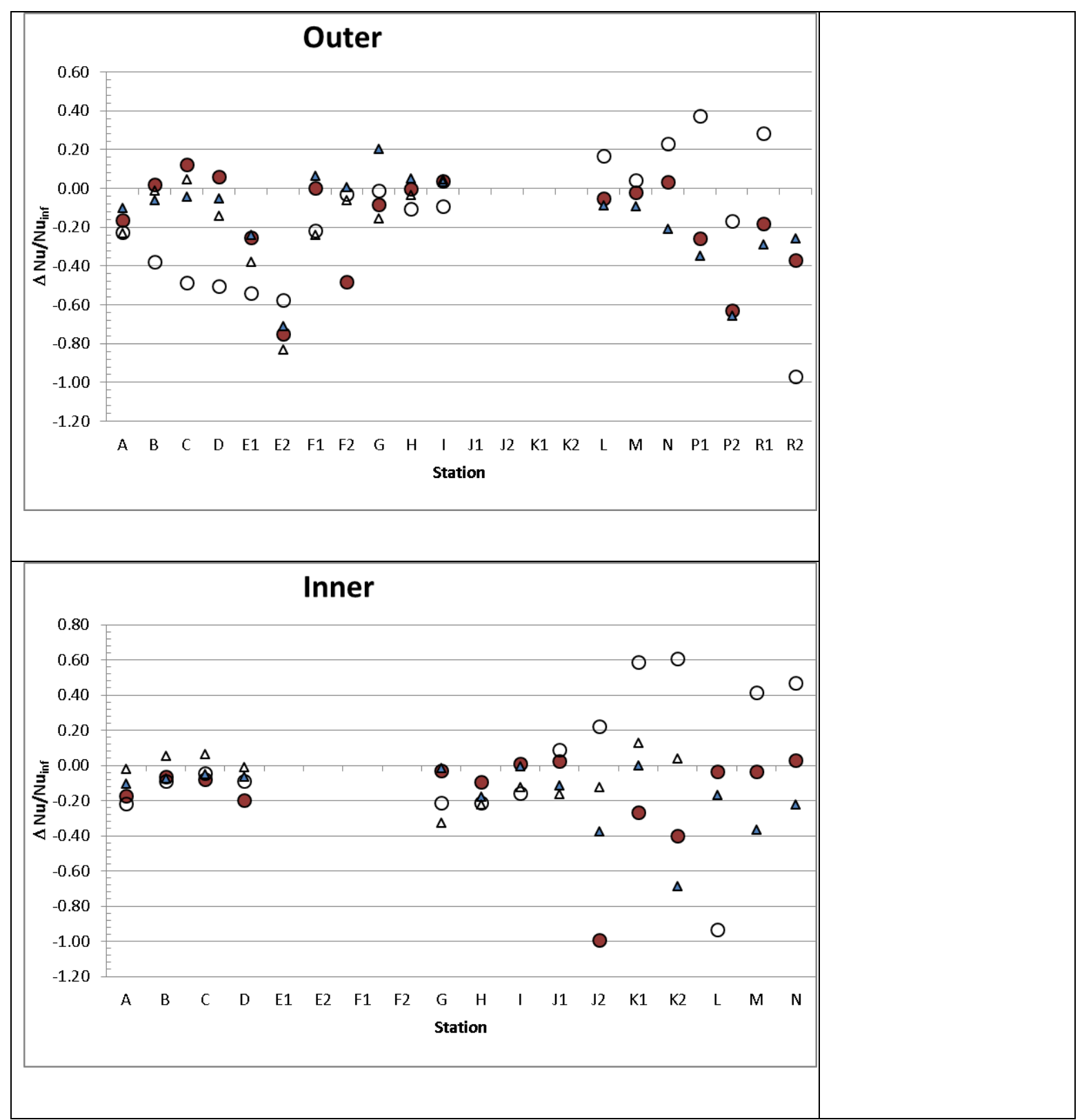

Figure 52. Reynolds number impact on passage heat transfer

Keep in mind that the experimental error cited in the host documentation increases to $~ 7 \%$ after station $\mathrm{K}$, in the final heated leg. Excluding the outer sidewall and data points after station $\mathrm{K}$, the variation in heat transfer due to Reynolds number change is well represented. The outer sidewall exhibits noticeable mismatch between experimental result and CFD for the turbulated geometry in the $1^{\text {st }}$ leg. 
HOST documentation indicates that relaminarization is likely to occur at the low Reynolds operating point $(\mathrm{Re}=12,500)$. The CFD SST model used for this research assumes fully turbulent flow.

\subsubsection{High Rotation Number Condition}

Simulations 9 and 10 in the HOST validation test suite exhibit a rotation number of 0.34 compared to the baseline Rotation number, 0.24 . These high rotation cases were included in the validation to assess the ability of the model to capture the impact of increased rotational speed relative to the bulk flow speed.

Heat transfer along the passage for the smooth walled geometry is illustrated in Figure 53.

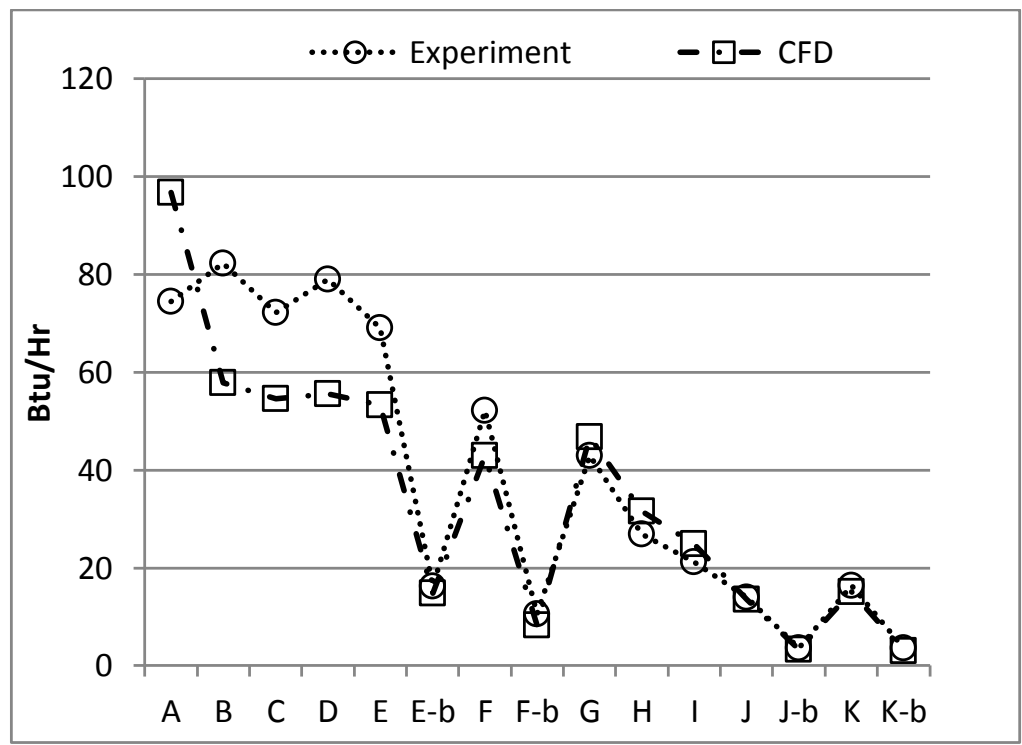

Figure 53. Heat transfer by station along HOST passage for simulation 9 
Consistently, we see a large discrepancy in heating in the first leg of the smooth walled passage. The impact of the plenum and screen used in the HOST experiment is clearly not captured in the CFD model inlet condition. The modelling approach used in this research would best be used in combination with a more precise inlet condition, or a domain that extends further upstream relative to the area of interest than in the current simulation. Downstream of the first bend, excellent data match is achieved.

Passage heat transfer for the high rotation operating point with skewed turbulators (case 10) is illustrated in Figure 54.

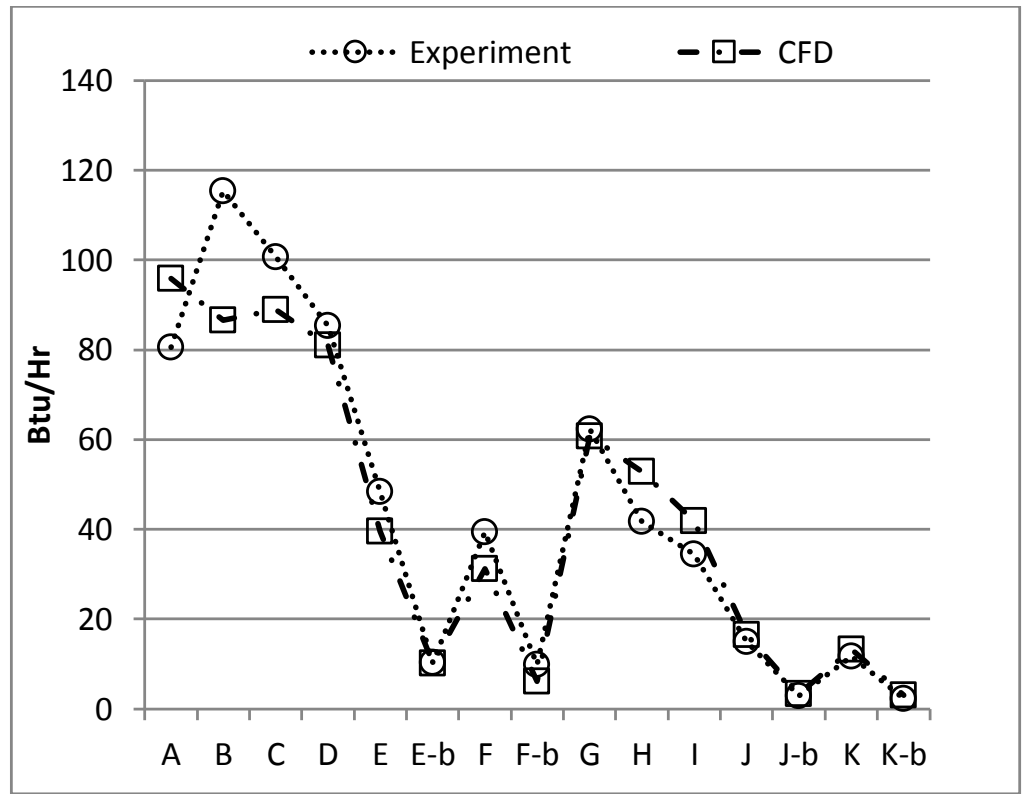

Figure 54. Heat transfer by station along HOST passage for simulation 10

The impact of the inlet condition mismatch compared to the experiment is quickly drowned out by the turbulator induced mixing in the passage, resulting in a smaller region of net heat transfer error than with smooth walls.

The impact of variation in Rotation number is illustrated in Figure 55 which compares, rotating baseline, and high Rotation \# for the smooth walled and skewed turbulator geometry. 


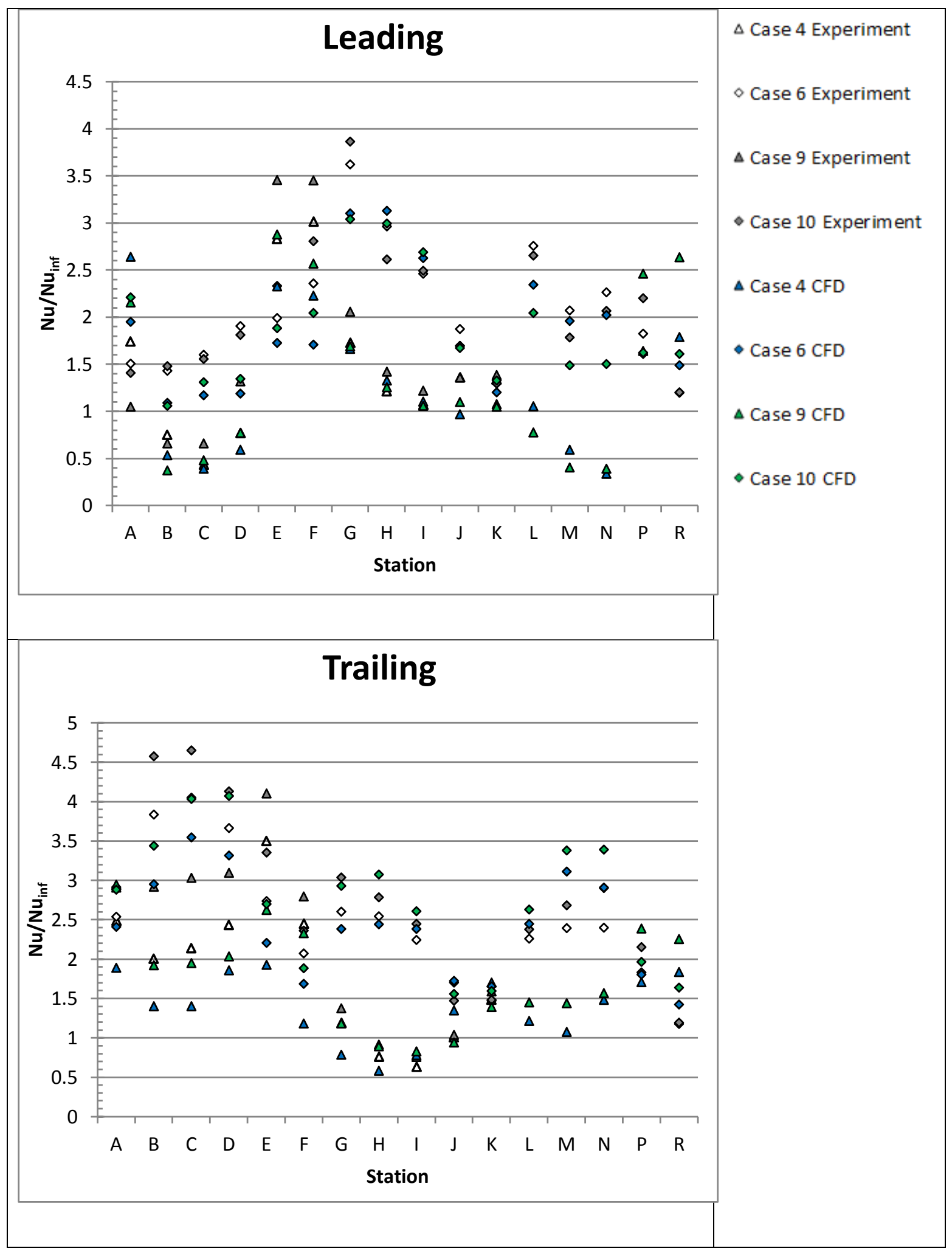




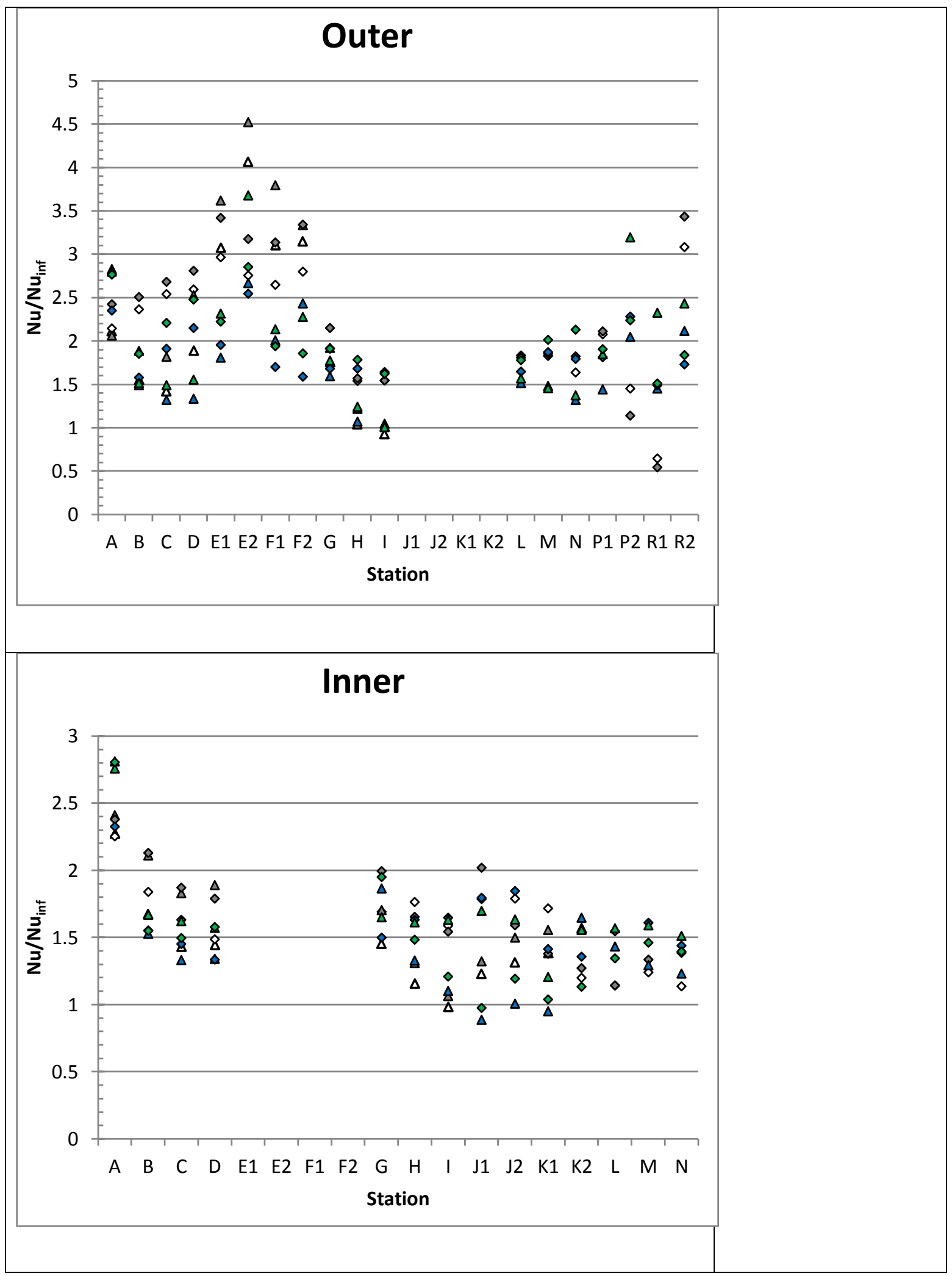

Figure 55. $\mathrm{Nu} / \mathrm{Nu}_{\infty}$ variation in passage at increased rotational speed 
The difference in Nusselt number between the baseline condition and high rotation condition for the smooth and skewed passages is examined by further processing the data. In order to isolate the impact of variations in Ro\# on wall heat transfer, $\Delta N u / N u_{\infty}$ is plotted in Figure 56. Increase in $N u / N u_{\infty}$ due to changing rotation number from 0.24 to 0.34 is elucidated. 


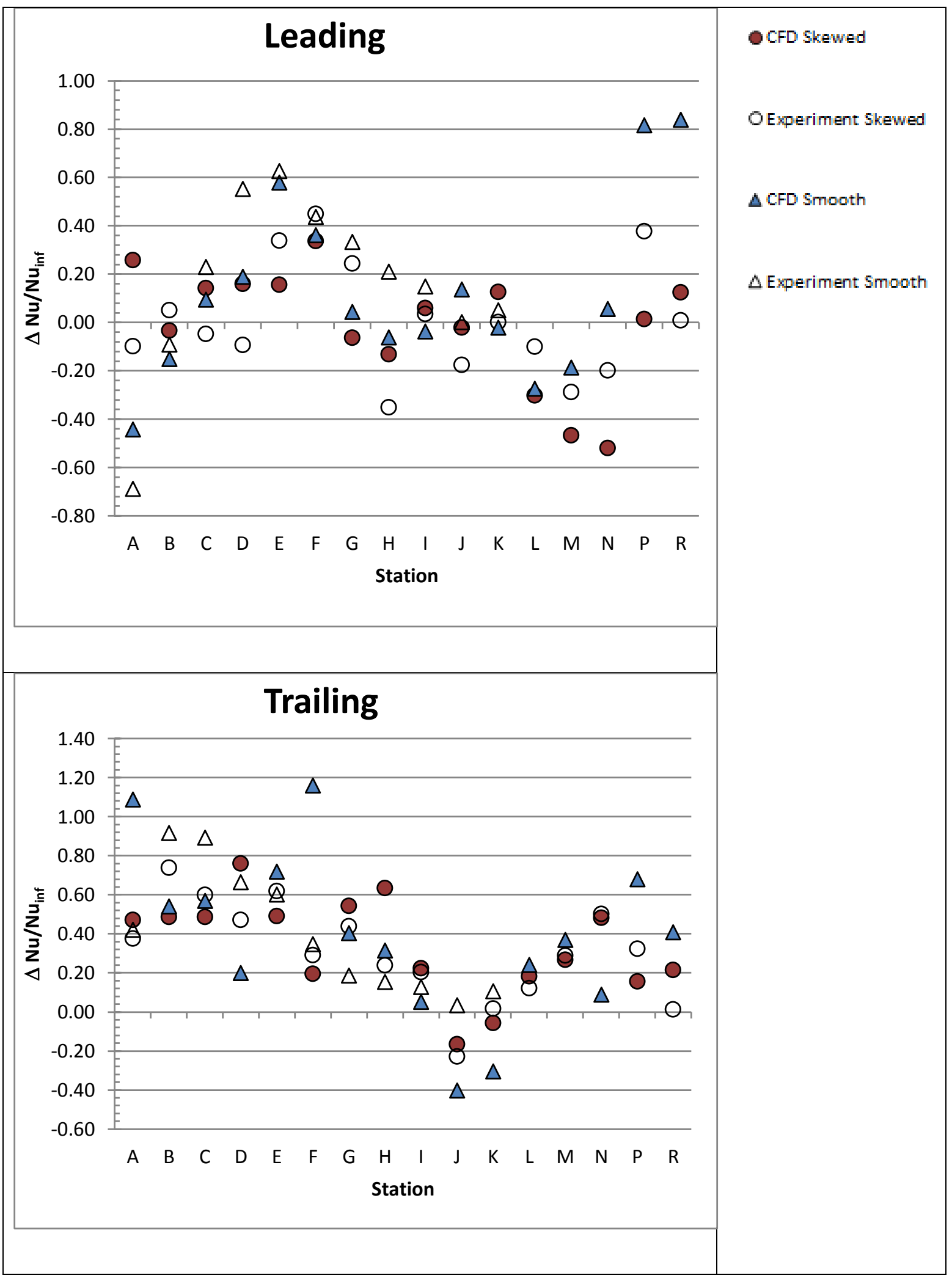




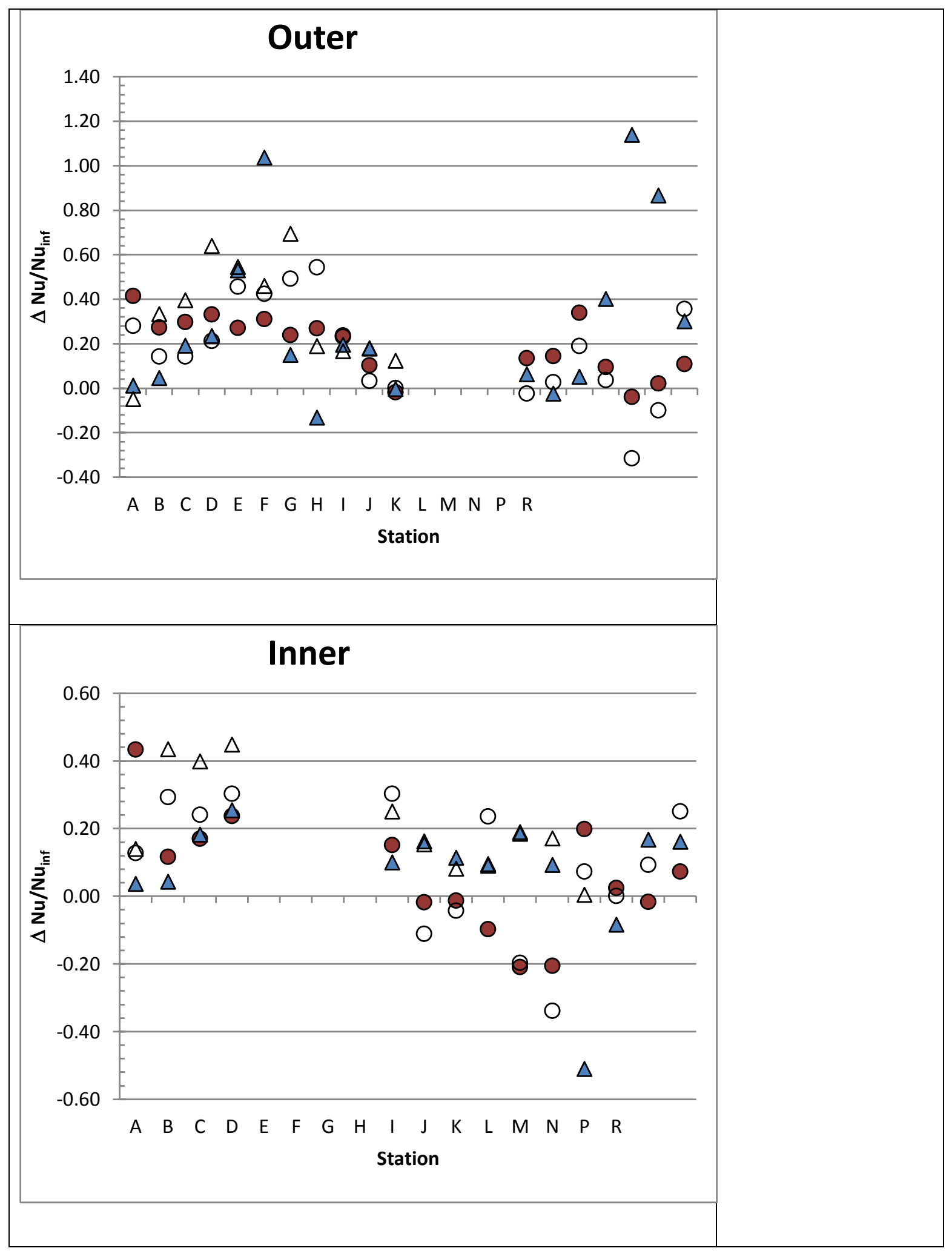

Figure 56. \% Impact of increased rotational speed on passage heat transfer 
Overall, the impact of varying rotation speed is captured by the CFD model. Small, complex turbulent structures exist in the flow, which make local data matching difficult. These localized effects result in deviations that are typically limited to individual plates. Simulations with the smooth walled geometry are especially susceptible to localized variations in heat transfer.

\subsubsection{Density Ratio Variation}

Heat pickup results with no turbulators and reduced density ratio, case 11 is illustrated along the passage in Figure 60.

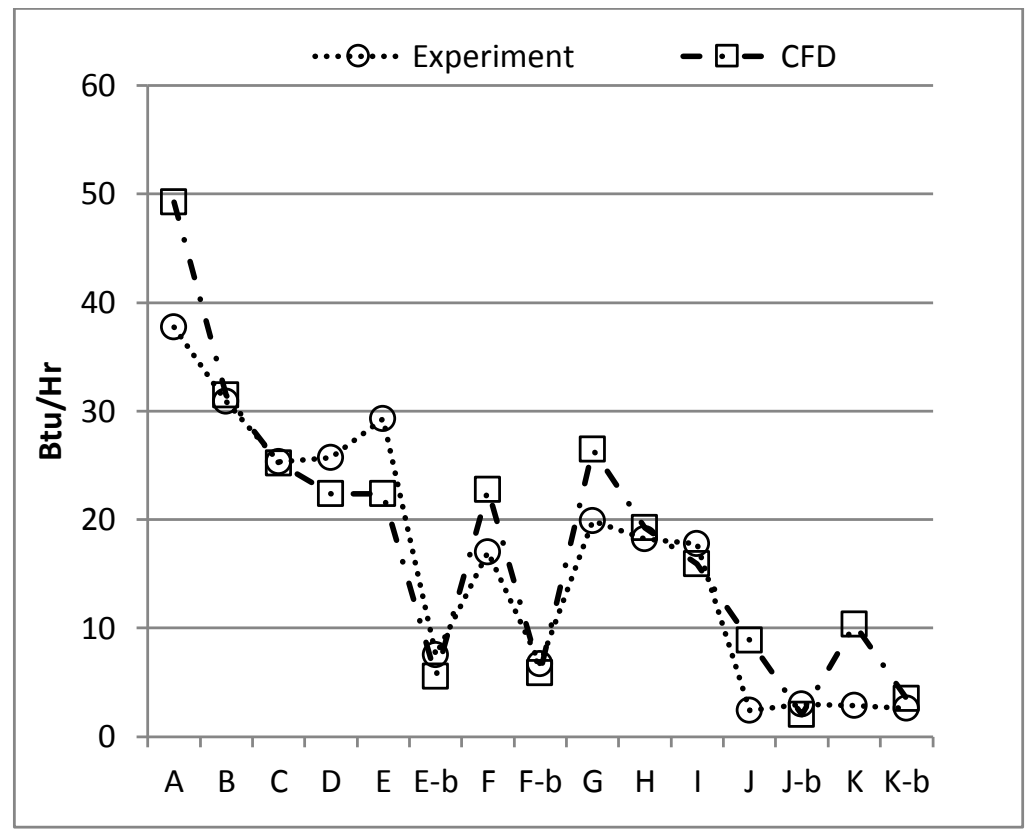

Figure 57. Heat transfer by station along HOST passage for simulation 11

Heat pickup results with skewed turbulators and reduced density ratio, case 12 is illustrated along the passage in Figure 60. 


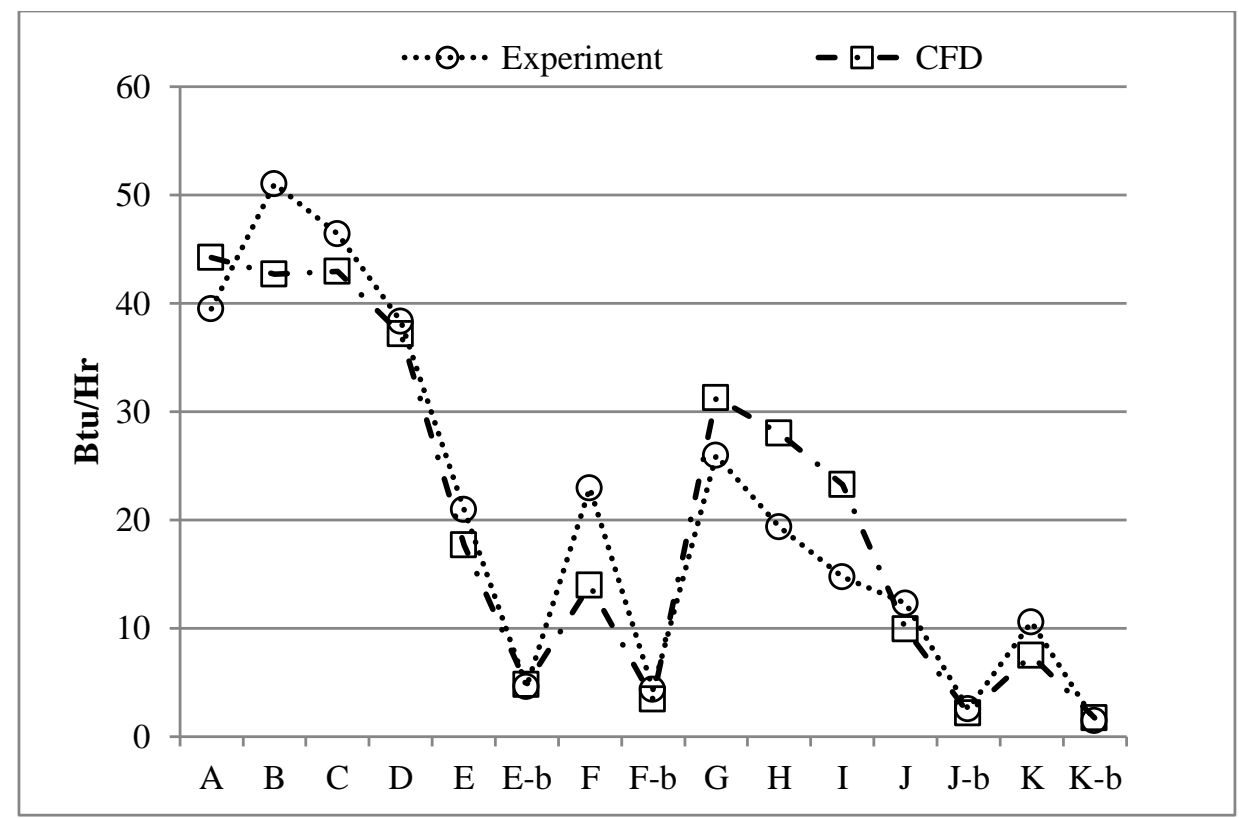

Figure 58. Heat transfer by station along HOST passage for simulation 12

Heat pickup results without turbulators and increased density ratio, case 14 is illustrated along the passage in Figure 59.

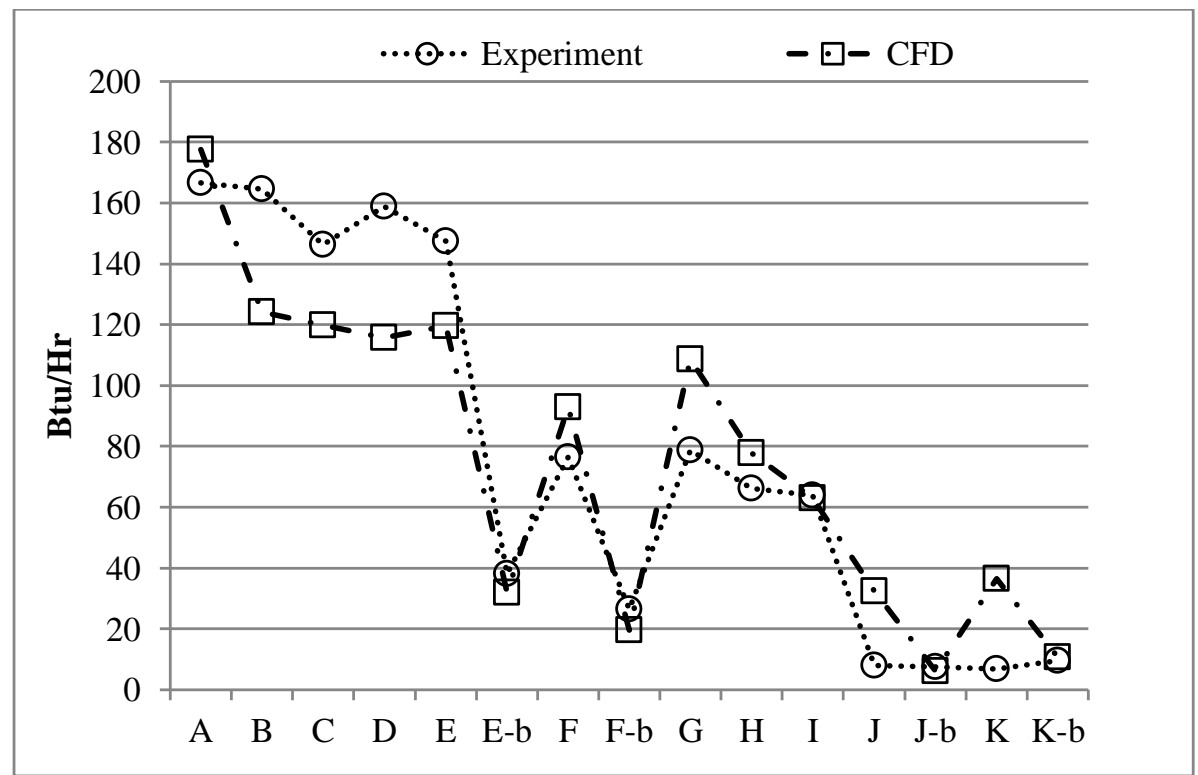

Figure 59. Heat transfer by station along HOST passage for simulation 13 
Heat pickup results with skewed turbulators and high density ratio, case 14 is illustrated along the passage in Figure 60.

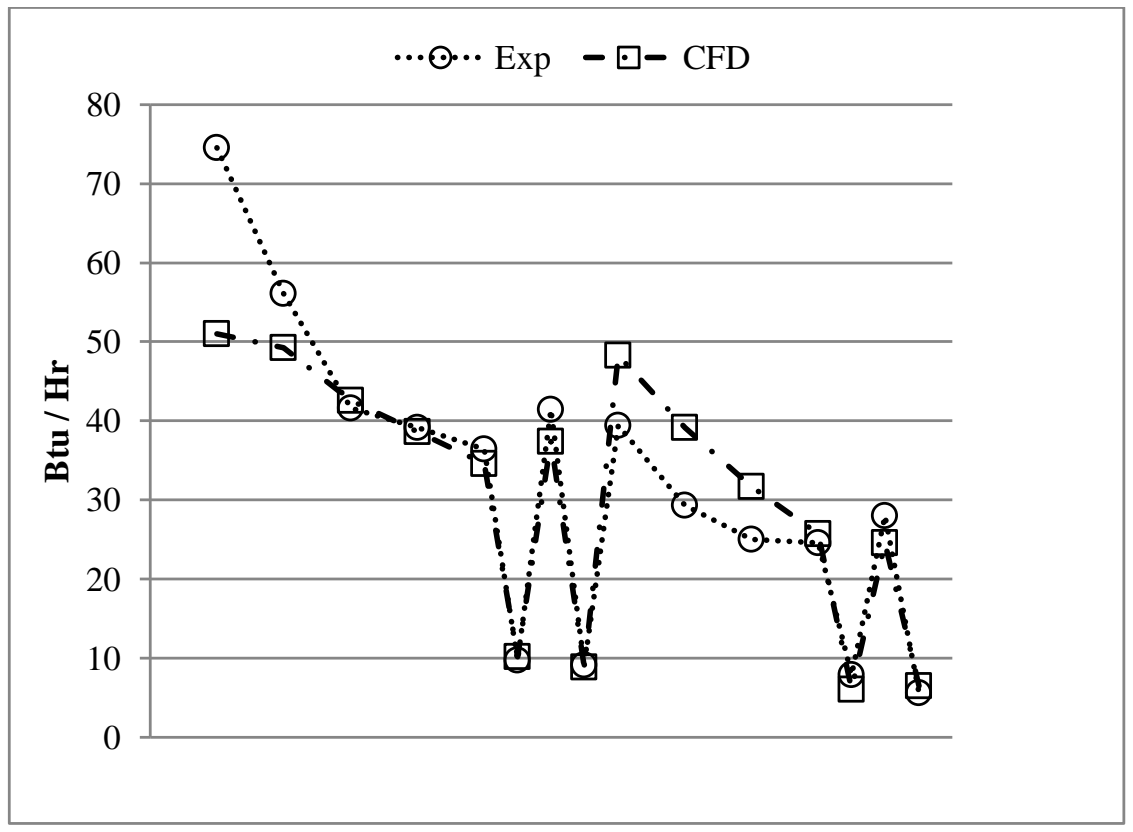

Figure 60. Heat transfer by station along HOST passage for simulation 14

The impact of Density ratio variation for the smooth walled passage geometry is documented in Figure 61. Case 11, 12, 13, and 14 are included. 


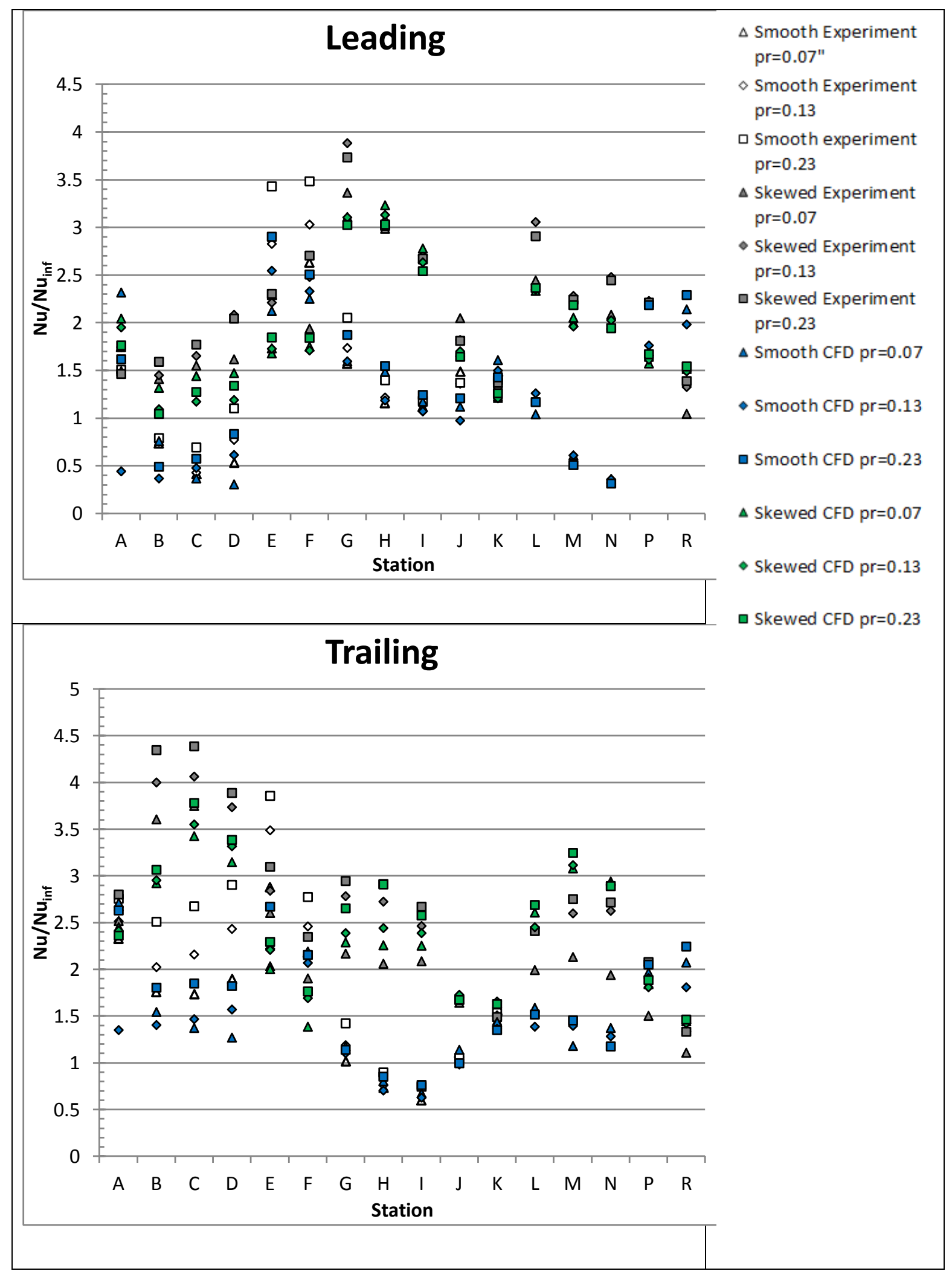

97 


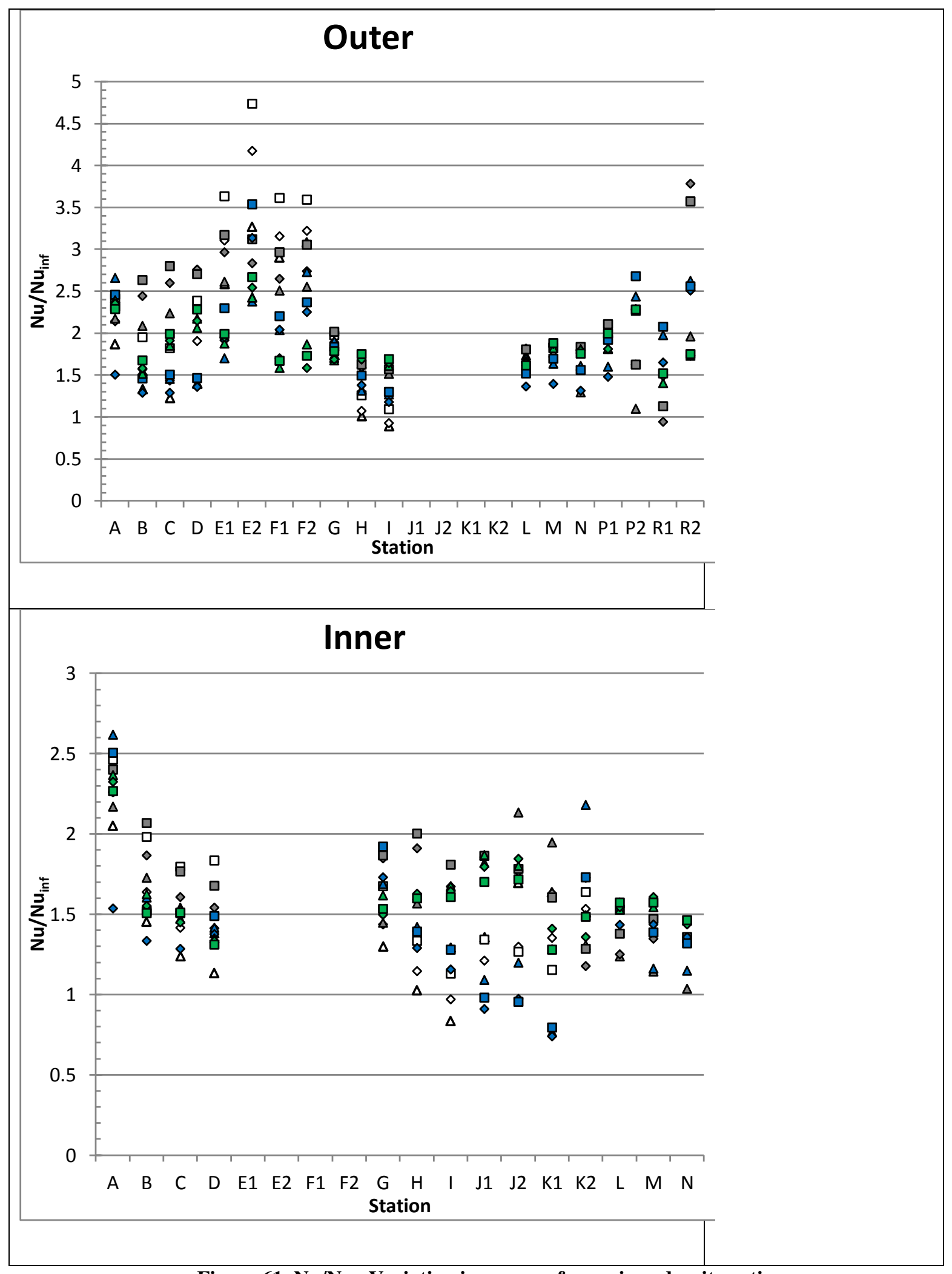

Figure 61. $\mathrm{Nu} / \mathrm{Nu}_{\infty}$ Variation in passage for various density ratios 
Further processing of the large dataset generated for HOST validation is undertaken to evaluate model sensitivity to changes in passage operating point.

\subsubsection{HOST Validation Summary}

Overall the validation of CFD data against HOST experimental data indicates a sensitivity of the CFD model to all of the primary factors that determine heat transfer in a rotating passage.

Combined heat transfer of all 4 walls matches experiment much more consistently than the single plate results, indicating that small local flow structures are not as well represented by the model as is the bulk impact of flow features and operating point on total heat exchange.

Entrance effect, and flow behavior entering and exiting the bends represent the greatest difficulty for the CFD model. An accurate representation of passage entry effects or a sufficient model runin to separate the area of interest from the inlet boundary is critical when modelling internal cooling to mitigate the sensitivity of calculation to assumptions made at the inlet. Bend entry and exit are driven by complex separated flow. RANS models such as SST are commercially applicable to such flows, but research has indicated that a more explicit Navier-Stokes simulation, such as L.E.S. can better predict free shear and turbulence behavior. The current CFD model accuracy is generally bounded by $\pm 20 \%$ error. LES based modelling could likely improve the accuracy of the model, but at great cost, outside the scope of current effort. Current practice relies on correlations for heat transfer predictions which typically exhibit error on the order of the current CFD model.

\subsection{Texas A\&M Experiment CFD Validation}

The simulation methodology developed for the HOST validation was also applied to the data set described in [5]. This data set is based on more modern instrumentation, with a more precise description provided of the test article geometry. 
Mesh density is based on results of the HOST program grid independence study. A block structured hexahedral mesh is generated which features about 9 million elements. The geometry exhibits quarter symmetry so a quarter sector mesh is generated and smoothed, then instanced to create the full domain mesh.

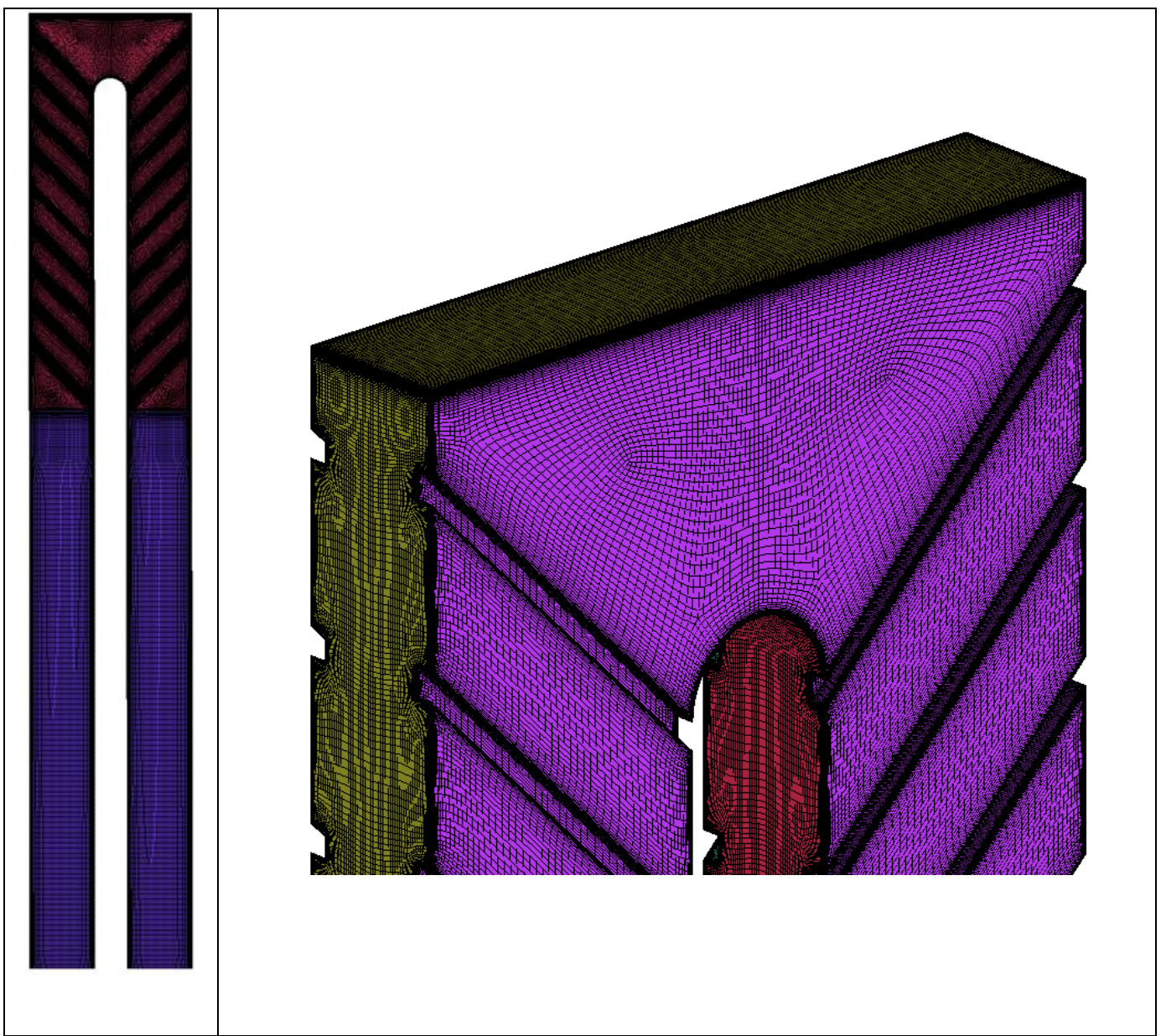

Figure 62. Mesh illustration for passage

Mesh density is targeted toward the mesh size chosen for HOST simulation based on the grid independence study performed. The experimental operating point validated against is outlined in Table 13. 
Table 13. J.C. Han Validation Operating Point

\begin{tabular}{|c|c|}
\hline Parameter & Value \\
\hline Re & 25,000 \\
\hline Ro & 0.21 \\
\hline Aspect Ratio & $2: 1$ \\
\hline Turbulators & 45 degree skewed \\
\hline
\end{tabular}

The inlet condition described for the experimental rig [5] is difficult to replicate in CFD. Other documentation for internal cooling experiments from Texas A\&M describe the inlet as a feed hole that is drilled into the base of the first leg which flows orthogonal to the leg direction. This approach is taken to provide a sudden inlet condition which does not carry developed rotational effects into the domain. The specific inlet geometry of this test is not described explicitly. An unheated section, purple in Figure 62, is included upstream of the heated section to create a partially developed flow profile in the experiment and CFD. An appreciable mismatch is observed, however, in the first heated section between the CFD and experiment. The heat transfer in the experimental rig is enhanced at the first plate relative to the others, suggesting that the flow was in fact not fully developed at that point.

Figure 63 compares the leading and trailing wall heat transfer within the passage between CFD and experiment. 


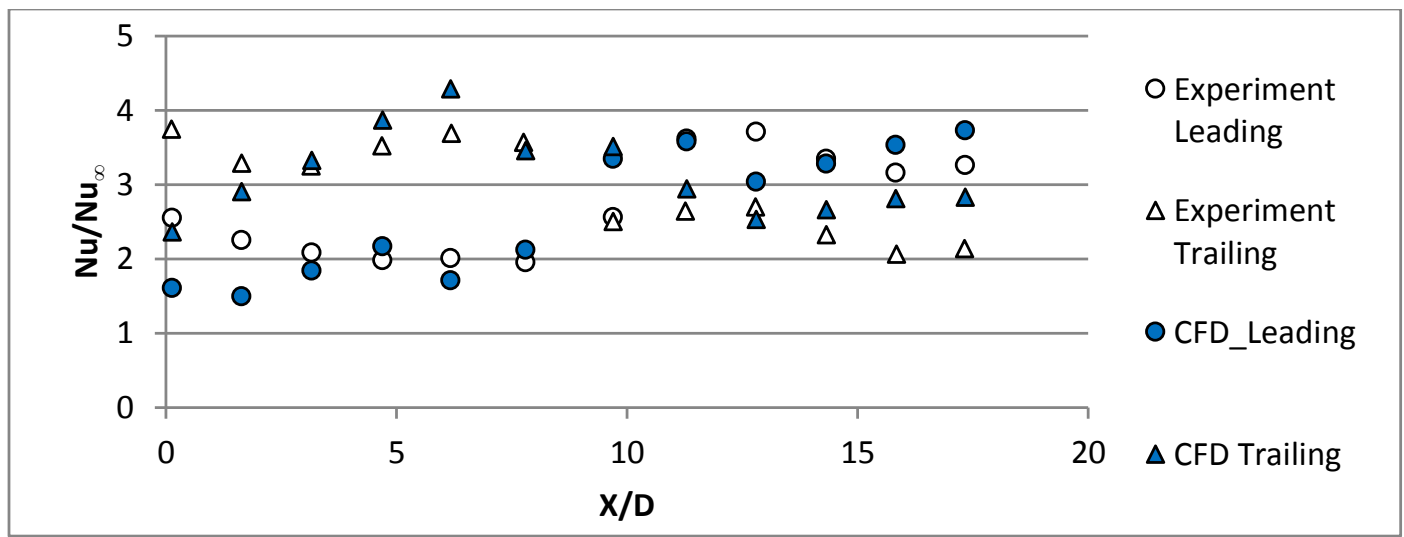

Figure 63. Nusselt number along passage for CFD and Experiment

Good agreement is observed away from the inlet, outlet and bend exit. Variations from the experiment at the first and last heated sections are likely due to differences between the treatment of the inlet and outlet in the CFD model and the experimental rig. CFD also predicts a higher level of heat transfer on both walls at the bend exit than measured in the experiment. The bend exit appears consistently to be the most difficult region to model due to large scale separation and coupling of various unstable physical phenomena.

Because the rig geometry is perfectly symmetric, heat transfer variations between the leading and trailing walls can be attributed entirely to rotation effects. The difference in Nusselt number between the leading and trailing wall is illustrated for the CFD and experimental data in Figure 64. A positive value corresponds to trailing wall heat transfer greater than leading wall heat transfer. 


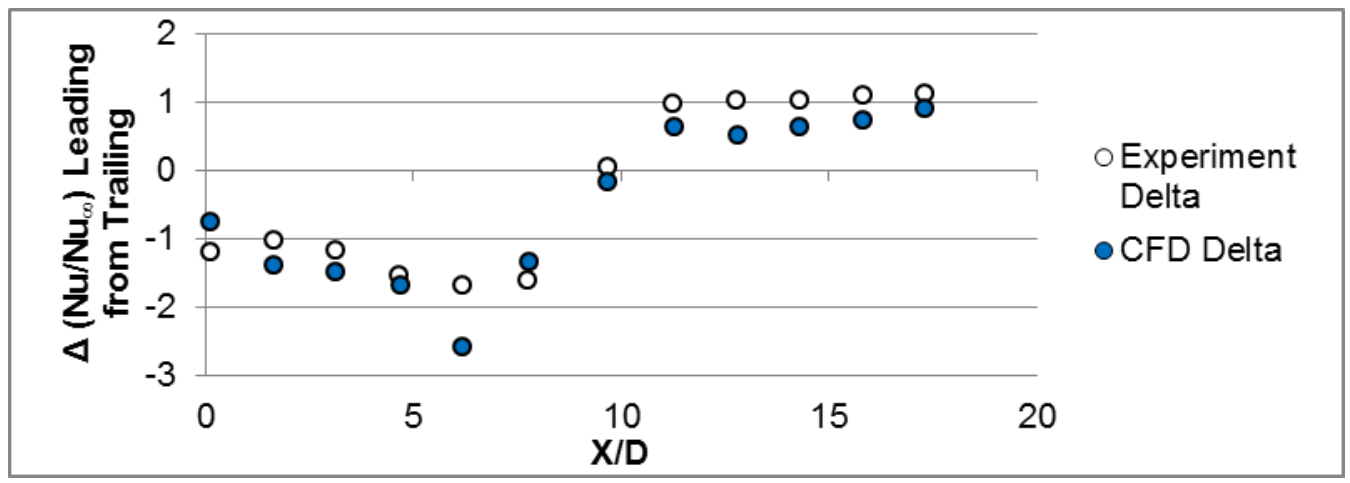

Figure 64. Difference between Leading and Trailing Nusselt number ratio due to rotation

The impact of rotation appears consistently reproduces by the CFD model with respect to the experiment. Aside from an overestimation of the impact leading into the bend at the fifth data point, the augmentation of heat transfer due to rotation is accurately modelled.

The percent difference in Nusselt number ratio at each location is presented in Figure 65.

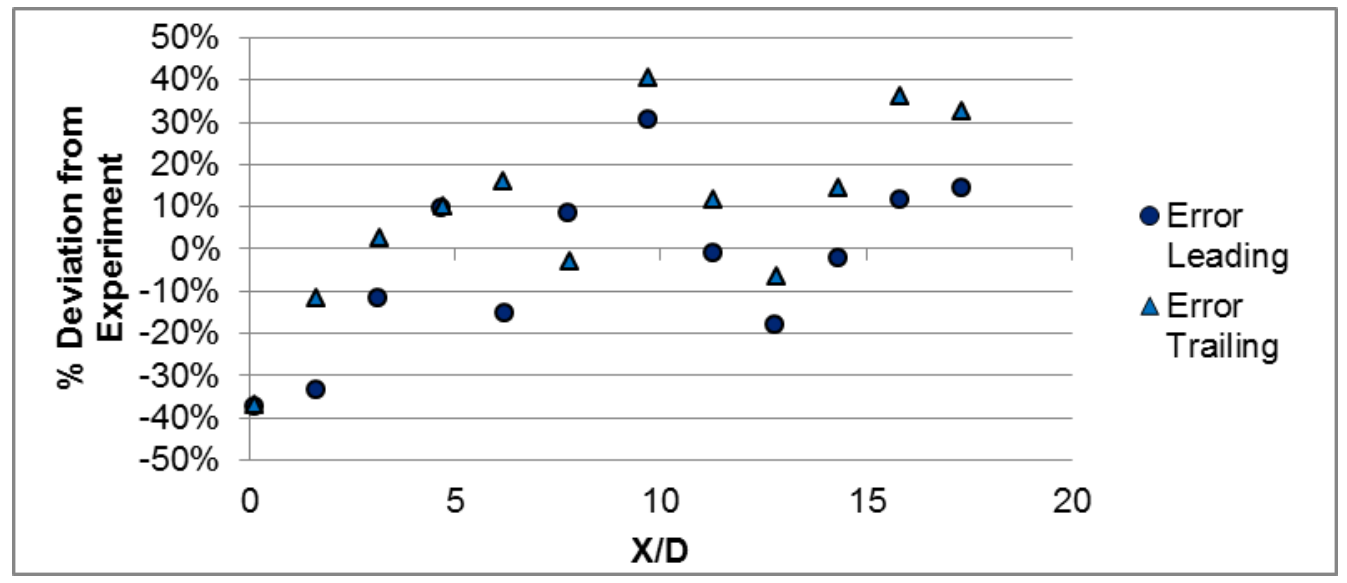

Figure 65. \% Deviation between experimental and CFD predicted Nusselt Number

The observations are consistent with prior discussion of Figure 63. Early and late in the passage, a significant difference exists between the CFD and experiment, likely due to boundary conditions which don't perfectly represent the rig inlet and outlet which are not explicitly described.

Otherwise, the only data point which exceeds $20 \%$ error is immediately after the bend. 
Wall shear contours shown in Figure 66 illustrate the interaction with separated vortex cores and the wall downstream of the bend. High shear areas can be seen which attribute to over predicted heat transfer.

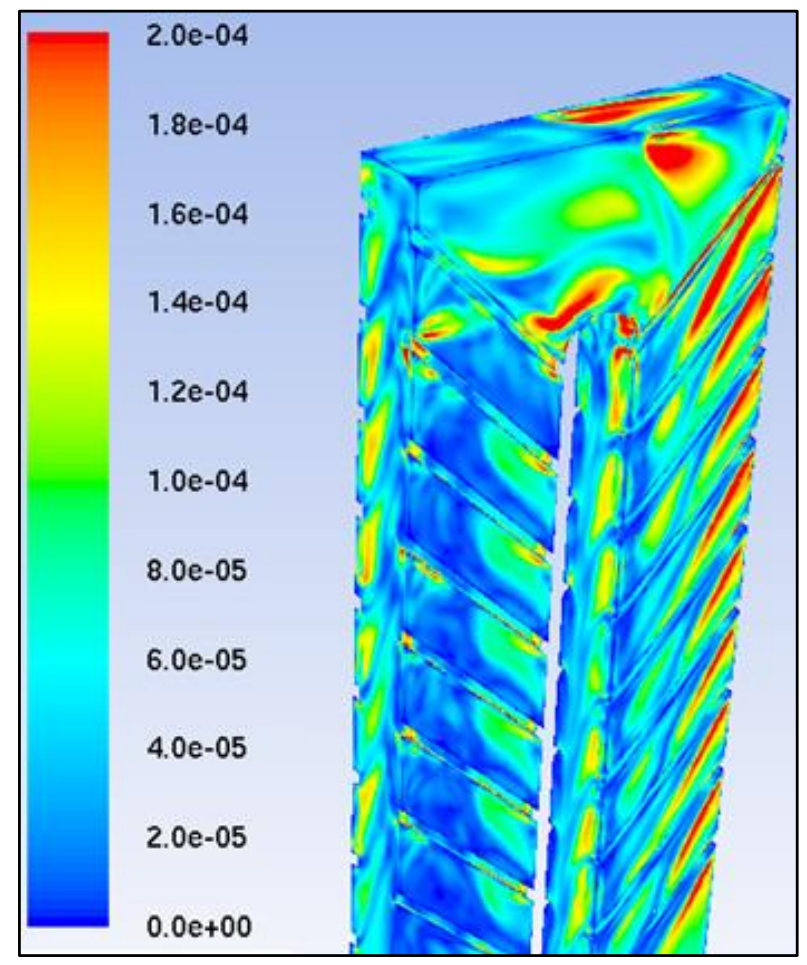

Figure 66. Wall shear in the bend region (in psi)

Overall, results of the added validation of the CFD model against the Texas A\&M experimental data is fairly consistent with the HOST data based validation effort. Care needs to be taken to accurately represent domain boundaries, or include a sufficient buffer region upstream and downstream of the region of interest to eliminate error which is attributed to inlet and outlet treatment. Additionally, local heat transfer immediately downstream of the bend is difficult to match in terms of localized heat transfer, but in a bulk sense

\subsection{University of Manchester Experiment CFD Validation}


As indicated in section 3.1.3, The Iacovides experiment is validated against CFD at a Reynolds number of 36,000 and a Rotation number of 0.4. The working fluid is water with Prandtl \# 6.99.

Before discussing results comparison between the CFD model and experimental results for this dataset, uncertainty in agreement between the operating points should be mentioned. The primary uncertainty in terms of agreement between the experimental test point and the operation point modelled in the CFD is the location of the axis of rotation. Figure 67 shows a cut plane of the passage mesh with an arbitrary axis of rotation illustrated.

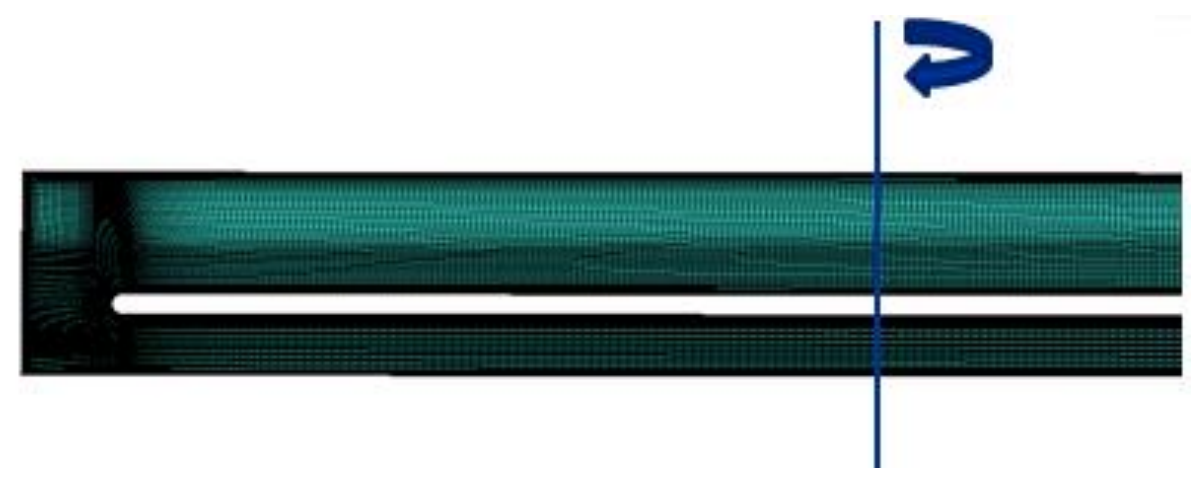

Figure 67. Mesh cut plane with axis of rotation indicated

For this analysis, the axis of rotation was placed halfway between the inlet and the bend tip. The actual location is not explicitly called out in the experimental documentation.

Because data is sampled near continuously, HTC, and Nusselt number published from the experiment are based on a continuously varying bulk temperature. Implementation of similar postprocessing of CFD results is prohibitive, so the CFD result are normalized by a constant reference temperature, specifically the mean of the inlet and exit temperature, $537{ }^{\circ} \mathrm{R}$. 


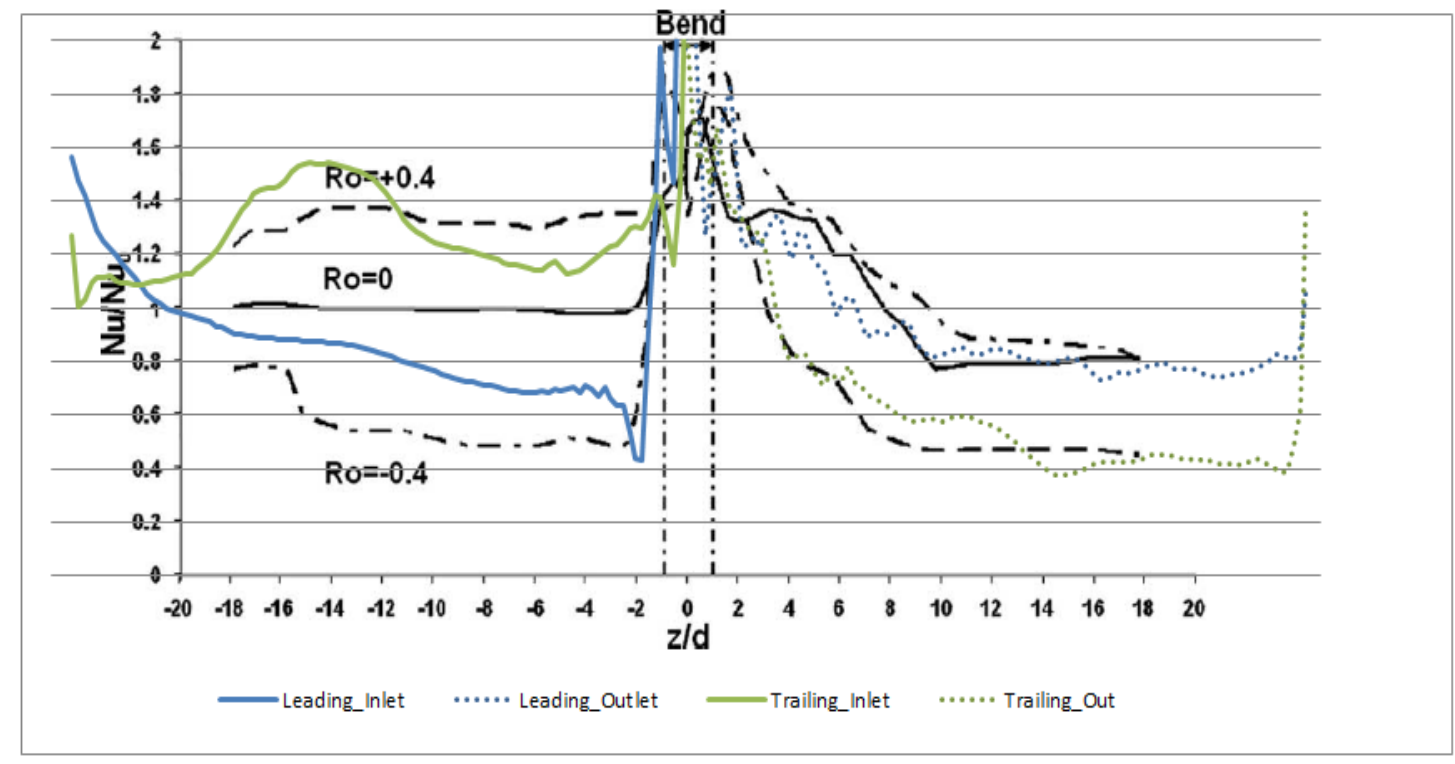

Figure 68. $\mathrm{Nu} / \mathrm{Nu}$ comparison between experiment and CFD

Experimental contours of wall Nusselt number are inherently time averaged. Because the behavior in the bend is inherently separated and unstable, Time averaged CFD results are necessary to make a good comparison. Unsteady CCT with time averaging of Nusselt number is performed in CFX. Figure 69 includes contours of Nusselt Number from the CFD simulation in color, and contours from the experiment as black bands. Recall that the CFD Nusselt Number is defined with a constant reference temperature, while the simulation uses a continuously varying reference temperature. 


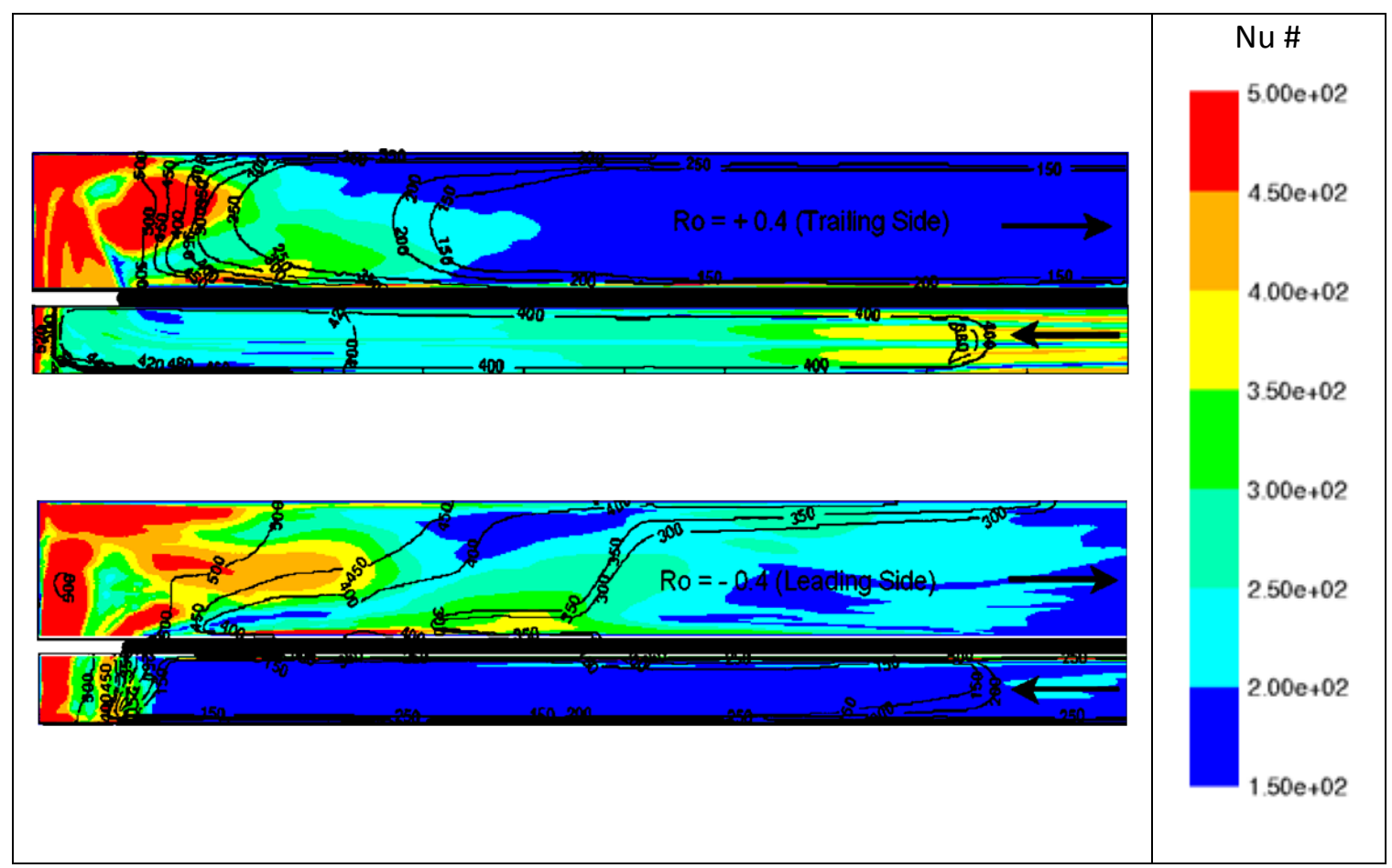

Figure 69. Experimental and CFD Nusselt Number Contours overlaid

On entry to the bend, The CFD model predicts the formation of flow structures similar to the experimental contour results. Exiting the bend however, specifics of heat transfer and location of hot spots are less similar. In a bulk sense the duration and magnitude of heat transfer augmentation exiting the bend matches well, but the interaction of vortices with the wall varies. This behavior is consistent with observations from validation efforts against HOST [2] [3] data and Texas A\&M [5] data. 


\section{CONCLUSIONS}

The validation exercise documented within this report evaluates the ability of RANS based CFD to predict convective heat transfer of cooling flow within a rotating serpentine passage.

Comparison of various experimental data sets to CFD results provides an understanding of the capabilities and limitations of this modelling approach for the physics involved in turbine cooling with serpentine passages.

With respect to initial modelling approach, note that Boundary conditions, mesh resolution, and turbulence modelling of the CFD model must be carefully considered, as these modelling choices can severely impact results. In the case of each of the three experimental programs considered, the treatment of the flow at the computational domain inlet proved a source of significant local error. Heat transfer at the entry of a passage is largely dependent on the flow state immediately upstream. A quality CFD model for a serpentine passage should either apply known turbulence levels and velocity profiles at the domain inlet, or include extra geometry upstream of the region of interest to allow the passage entry condition to develop within the model. In the case of this research, the inlet conditions and upstream geometry were not readily available.

The level of mesh sensitivity for turbulated serpentine passages demonstrated herein indicates the importance of performing a mesh sensitivity analysis for any CFD analysis. The physical length scales to be captured by CFD must be resolvable on the provided mesh. In the case of this analysis, the smooth block structured meshes compare very favorably to unstructured tetrahedral meshes in terms of efficiency. A 6 million element structured mesh is shown to achieve lower grid dependence than a tetrahedral mesh of 63 million elements. It is often thought that high order discretization eliminates any effective performance advantage of structured hexahedral meshes over tetrahedral meshes, but this research demonstrates otherwise. High order 
numerics reduce the impact of flow alignment of faces, but also note that a cube of a given edge length occupies over 8 times the volume of a perfect tetrahedron of the same edge length. This means that if you wish to resolve a given length scale, it requires roughly 8 times as many tetrahedra as hexahedra to occupy the same volume. Mesh sensitivity evaluation in conjunction with CFD analysis is an important check which greatly improves veracity of model results.

Another key modelling choice which demonstrated great impact on simulation results is turbulence model selection. Different turbulence models present different benefits. It is important to consider what physics are involved in a problem and understand what available models can capture those physics. The balance between model complexity and the fidelity of turbulence modelling is an important consideration for CFD analysis and of central focus to research. The 2 equation SST model is demonstrated to provide sufficient accuracy in this case without incurring the significant computational cost of more sophisticated models such as RSM or Large Eddy Simulation.

The experimental data utilized in this validation effort allowed for the evaluation of model accuracy with respect to bulk heat transfer and other key performance metrics for internal cooling, but a more complete and physically grounded validation may be possible with additional experimental data. The ideal experimental outputs for a comprehensive CFD model validation are listed below:

- Explicit documentation and characterization of inlet geometry

- Detailed dimensioned drawings sufficient to entirely re-create test article without assumptions

- High resolution 3-D velocity data at domain inlet to capture secondary flows

- High resolution turbulence intensity or turbulence kinetic energy and length scale data at the domain inlet 
- PIV (particle image velocimetry) data at bend entry, mid bend, and bend exit to understand secondary flow behavior and better understand turbulence model shortcomings

- Continuous wall shear data throughout heated section

It is understood that acquisition of the data outlined above is extremely difficult given modern techniques and the inertial loading associated with rotating turbomachinery. Consider the list above a wish list which, if achieved, would facilitate future CFD validation to more completely understand simulation performance. Particularly, the understanding of secondary flow structures throughout the bend at various operating points could provide significant insight into the interaction of the driving physics which impact turbine cooling flow. Turbulence model against experimental behavior of these interactions would be paramount in providing physically based rationale for selection of a given model and open the door to model studies to improve heat transfer predictions in various rotating machinery applications.

Internal cooling passages present an extremely difficult fluid dynamics problem. Considering the complexity of the flow and the level of error that is typically expected for Internal cooling heat transfer predictions, the CFD model validated herein provides a reliable means for the estimation for cooling passage heat transfer across a wide range of operating points. In general, the accuracy of the computational model is within $10 \%$ for heat transfer of a full passage, and $20 \%$ in terms of local features. This accuracy is consistent across variations in Reynolds number, rotation number, density ratio, and turbulator configuration.

This level of accuracy is within an acceptable range for turbine cooling predictions. The key benefit of the model compared to Experimental correlation is in extrapolation. Because CFD is based on the solution of a set of governing conservation equations, the physical viability is not limited to the range of operating points for which tests have been performed. Having evaluated the ability of the CFD model to capture the effects of the range of physical phenomena which 
impact internal cooling flow for rotating turbomachinery, we can simulate different internal cooling operating points with reasonable certainty in the results without performing additional experimentation. 


\section{BIBLIOGRAPHY}

1. Dual Solutions for Steady Laminar Flow Through a Curved Tube. Dennis, S.C.R. and Ng, Michael. 3, s.1. : Oxford Journals, 1982, Quarterly Journal of Mechanics and Applied Mathematics, Vol. 35, pp. 305-324.

2. Effects of Rotation on Coolant Passage Heat Transfer, Volume 1. T.J. Hajek, J.H. Wagner, B.V. Johnson, A.W. Higgins, and G.D. Steuber. NASA Contractor report 4396, Vol. I

3. Effects of Rotation on Coolant Passage Heat Transfer, Volume 2. B.V. Johnson, J.H. Wagner, G.D. Steuber. NASA Contractor Report 4396, Vol. II

4. Experimental study of thermal development in a rotating square-ended U-bend. H. Iacovides, D. Kounadis, Z. Xu. 2009, Experimental Thermal and Fluid Science Vol. 33 pp. 482-494

5. Heat Transfer in a Two-Pass Rectangular Rotating Channel with 45-deg Angled Rib

Turbulators. Gm S. Azad Mohammad J. Uddin, Je-Chin Han, Hee-Koo Moon, and Boris Glezer. April 2002, Journal of Turbomachinery Vol. 124 pp. 251-259

6. Fluent Theory Guide, version 15 ANSYS Software documentation

7. Nikuradse, J 1926. Untersucheungen uber die Geschwindigkeitsverteilung in turbulenten Stromungen. Ph.D. Thesis, University of Gottingen, Germany

8. Reynolds number dependence of mean flow structure in square duct turbulence. Alfredo Pinelli, Markus Uhlmann, Atsushi Sekimoto, and Genta Kawahara. Journal of Fluid Mechanics 644 pp 107-122

9. Numerical Study of Turbulent Secondary Flows in Curved Ducts. N. Hur, S. Thangam, C.G. Speziale. NASA Contractor Report 181830, April 1, 1989. 


\section{APPENDICES}

A: Section Cuts of Velocity for Select HOST Operating Points

Case 4
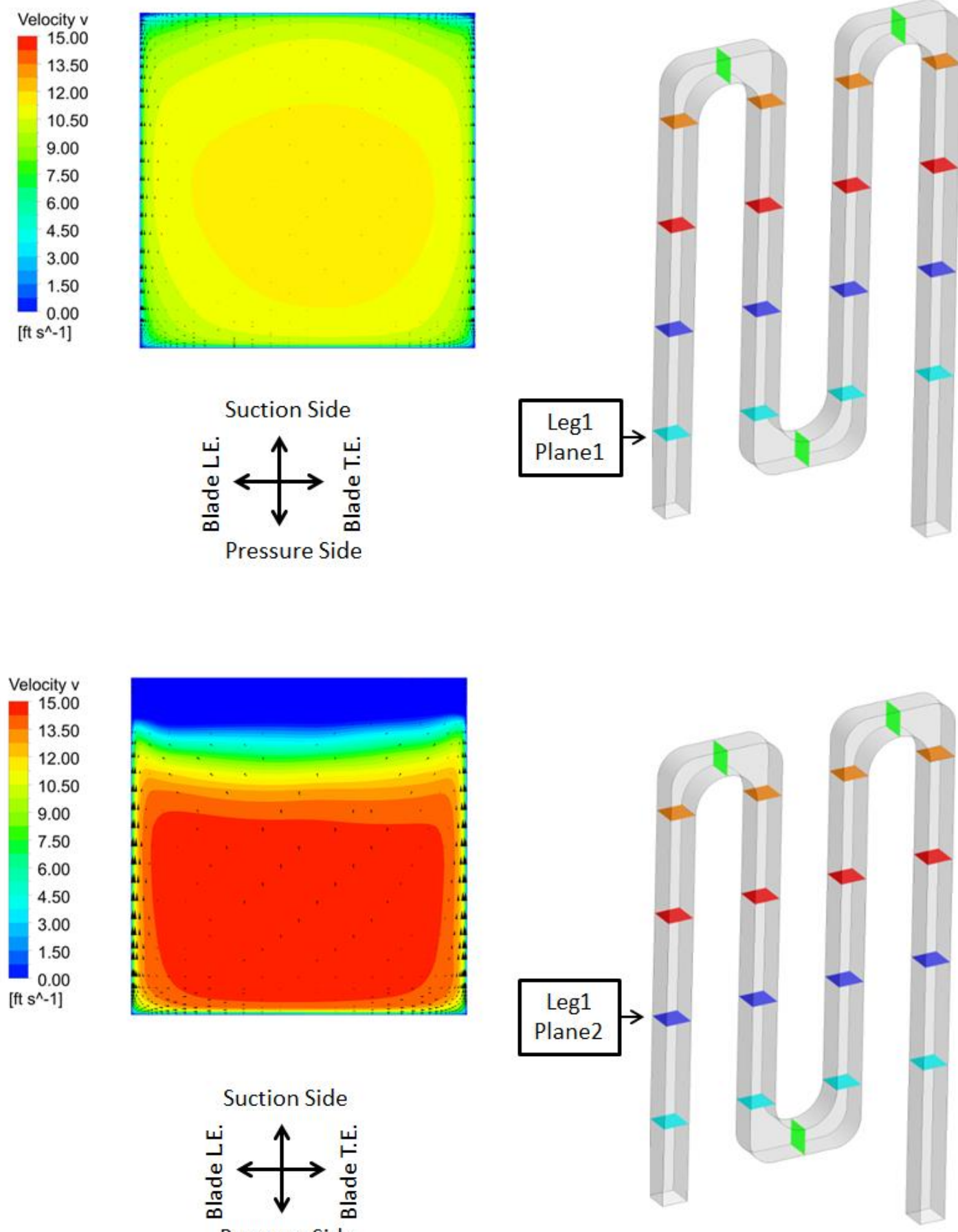

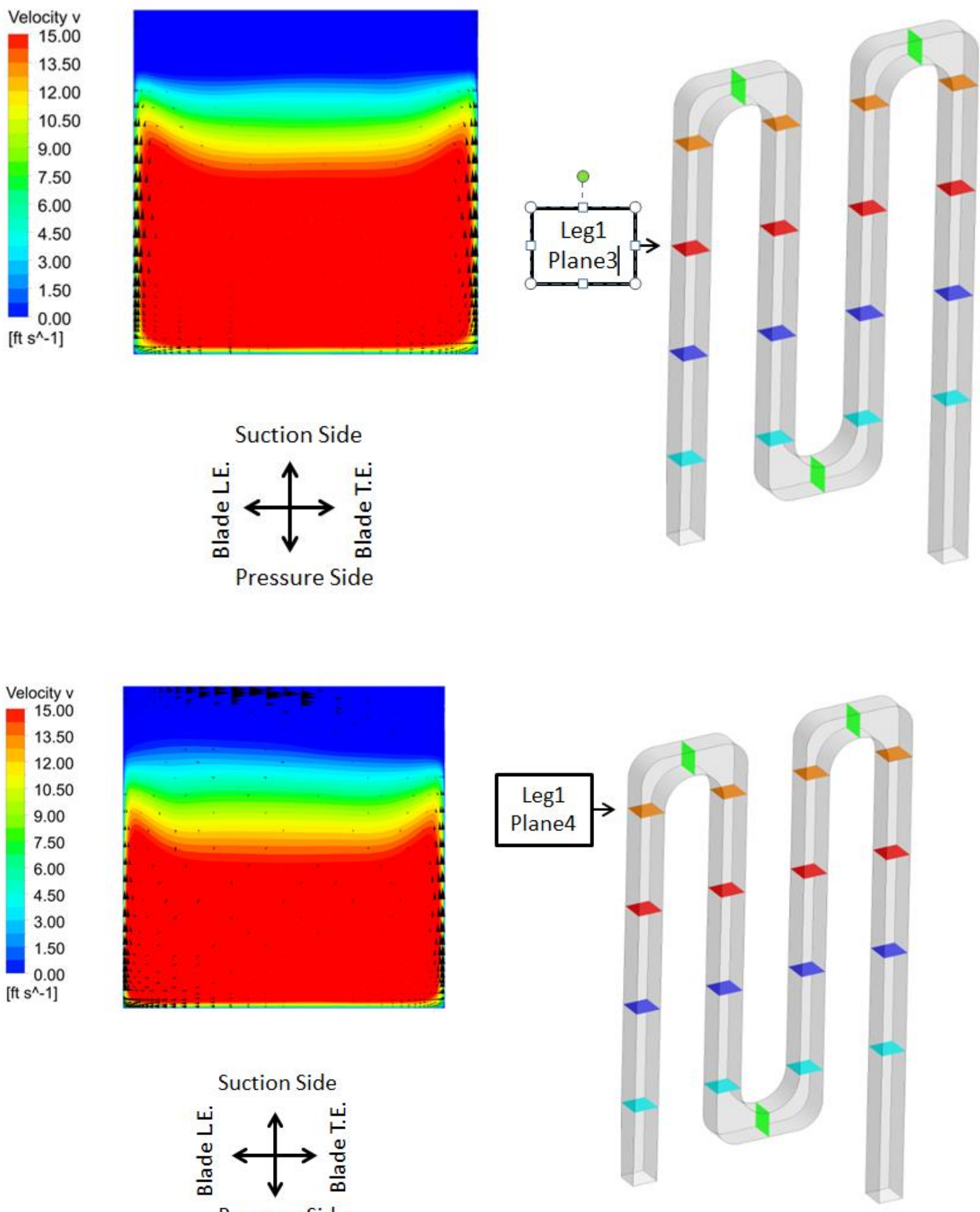

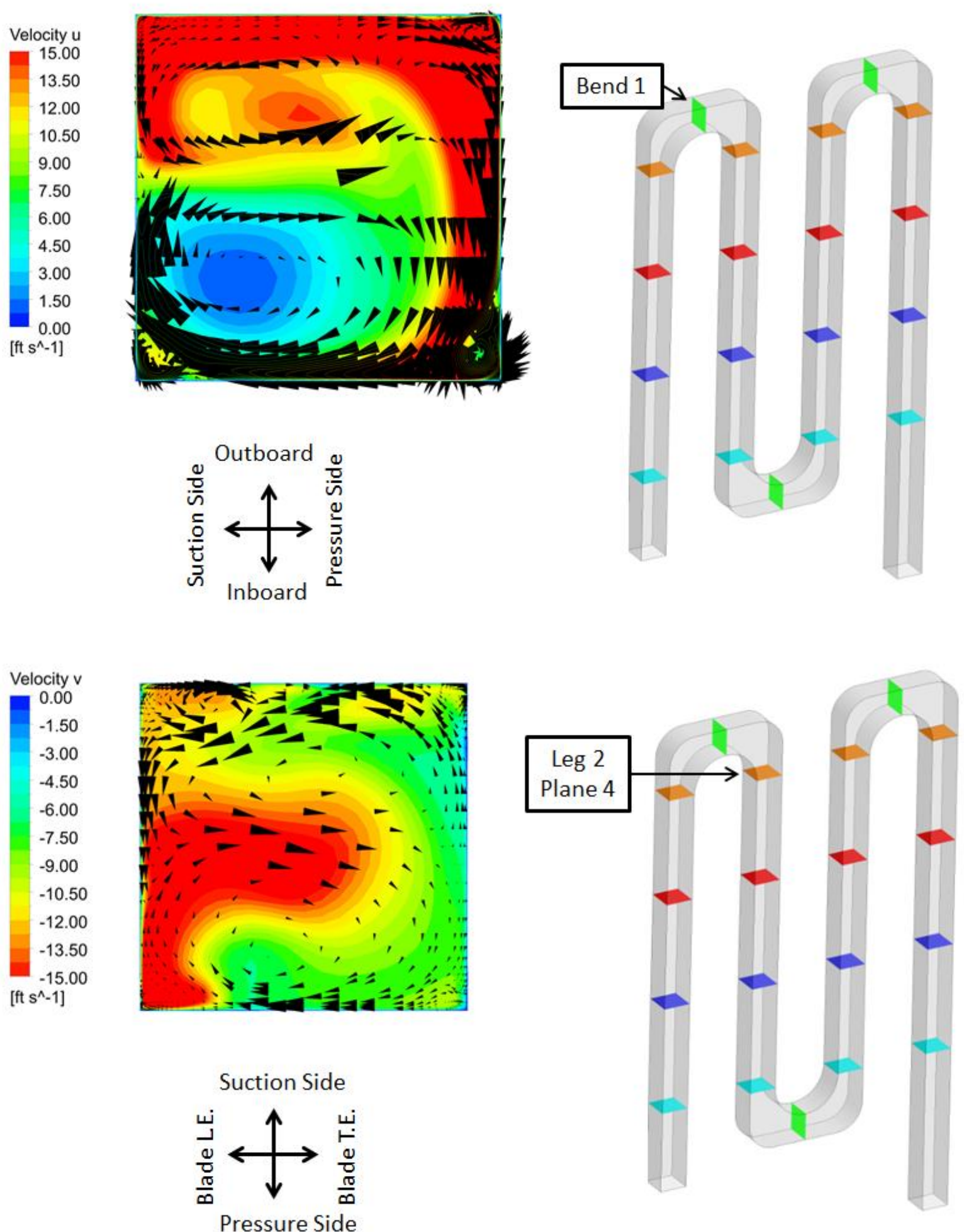

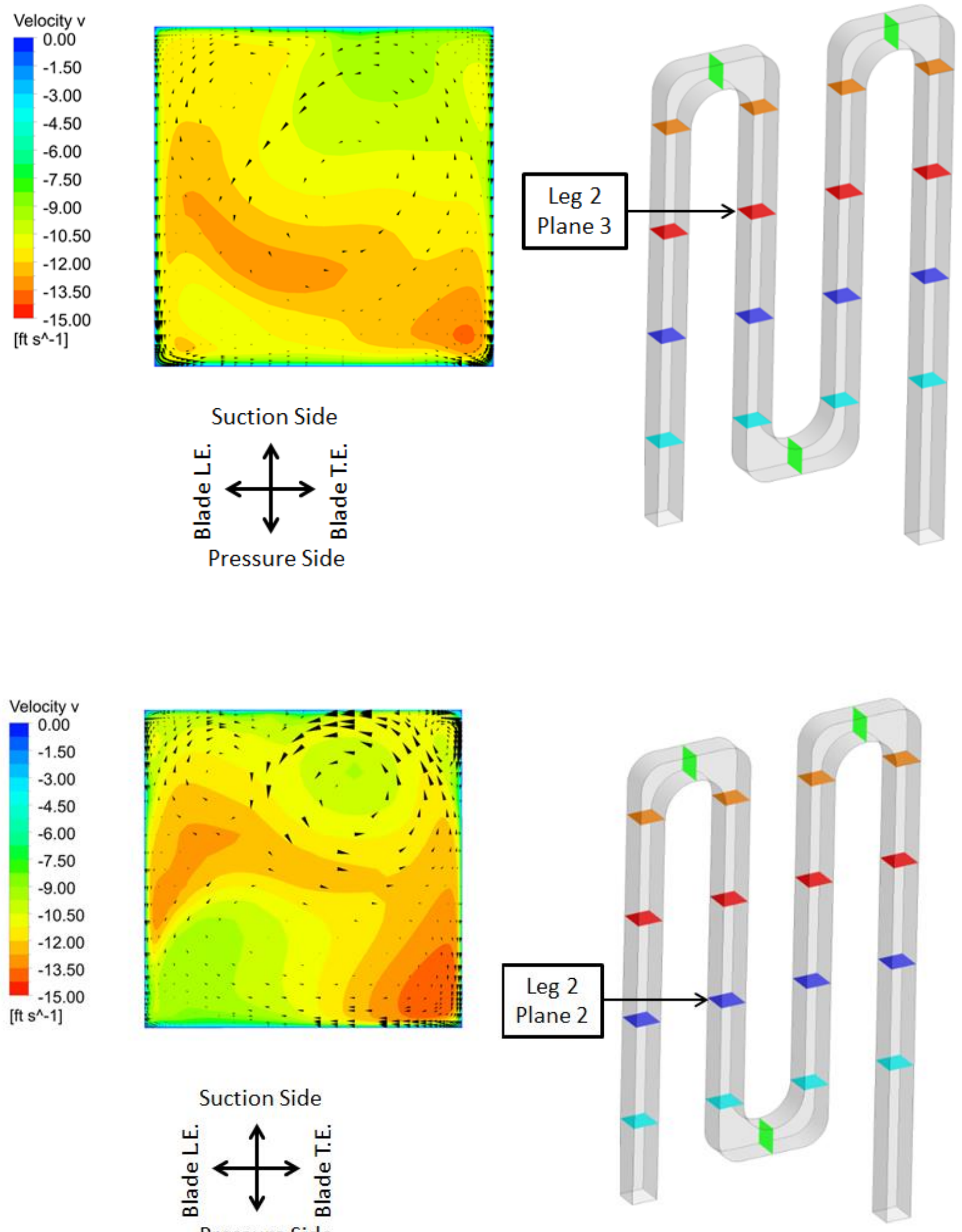

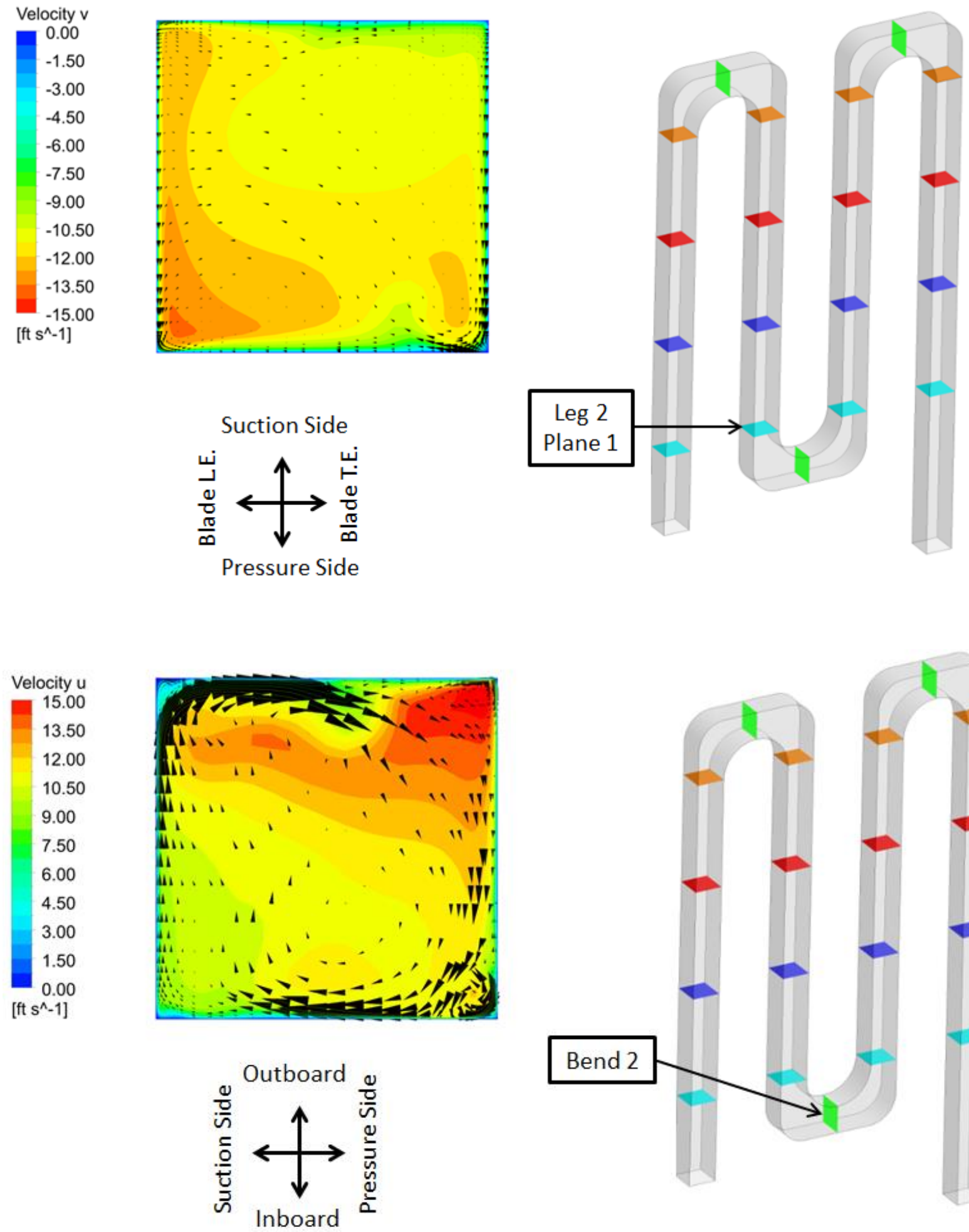

Bend 2
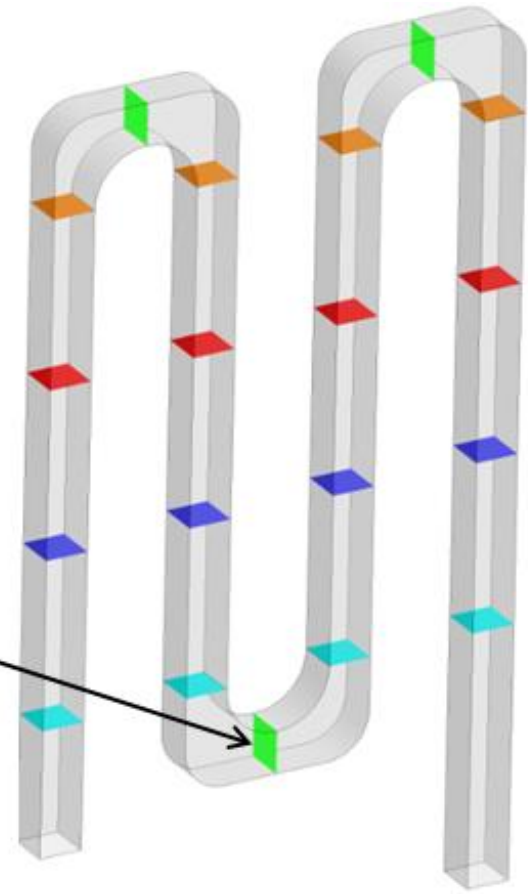

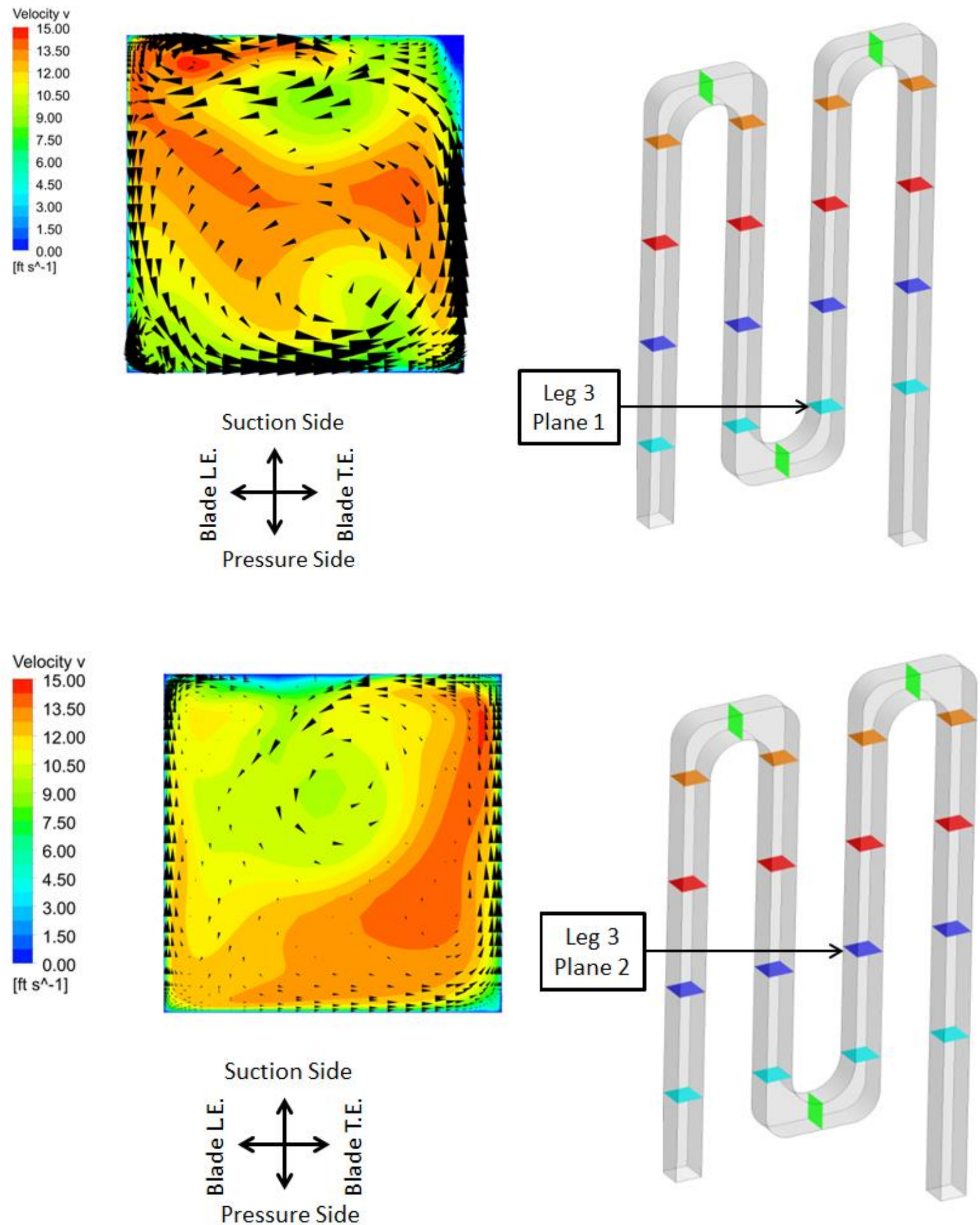

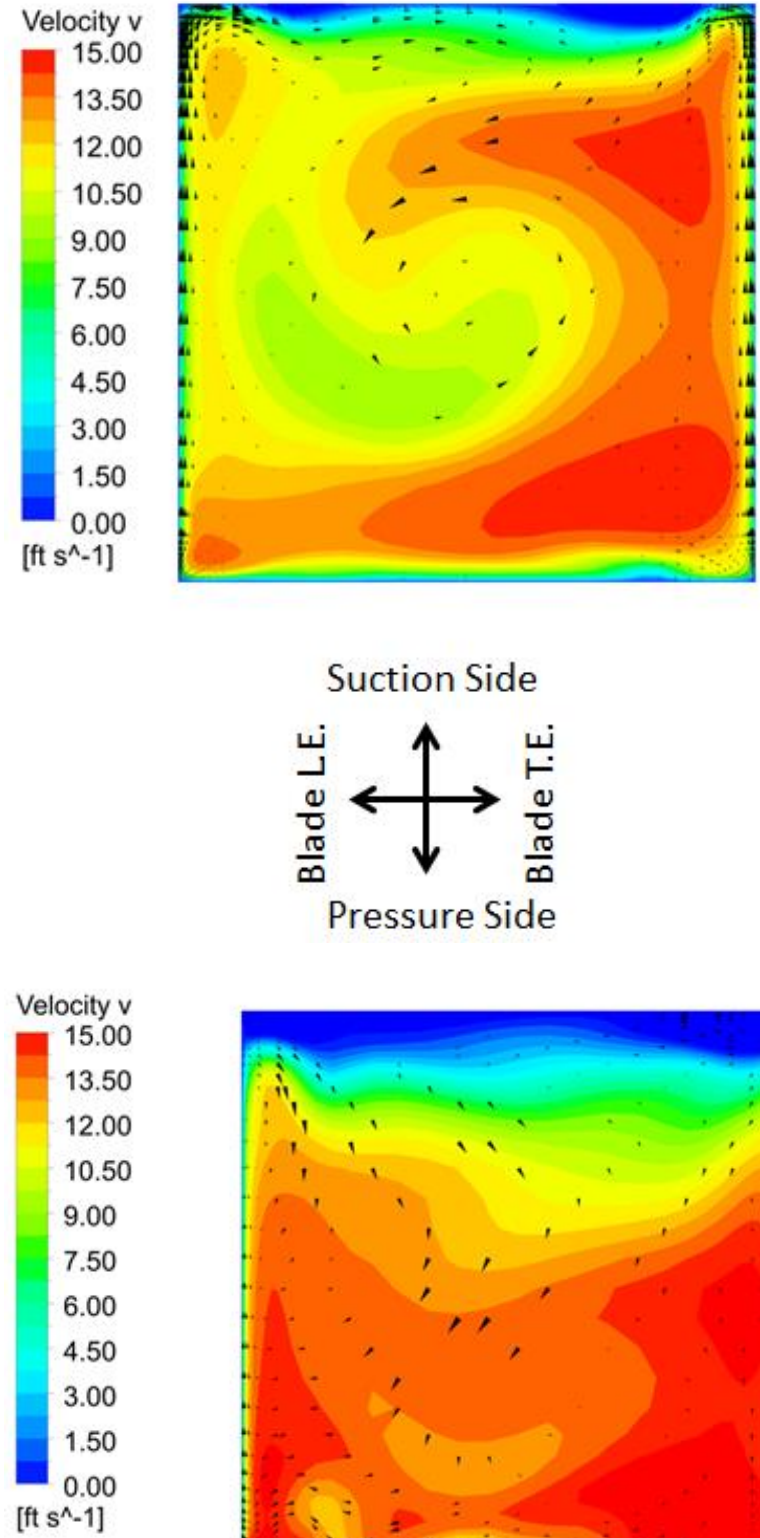
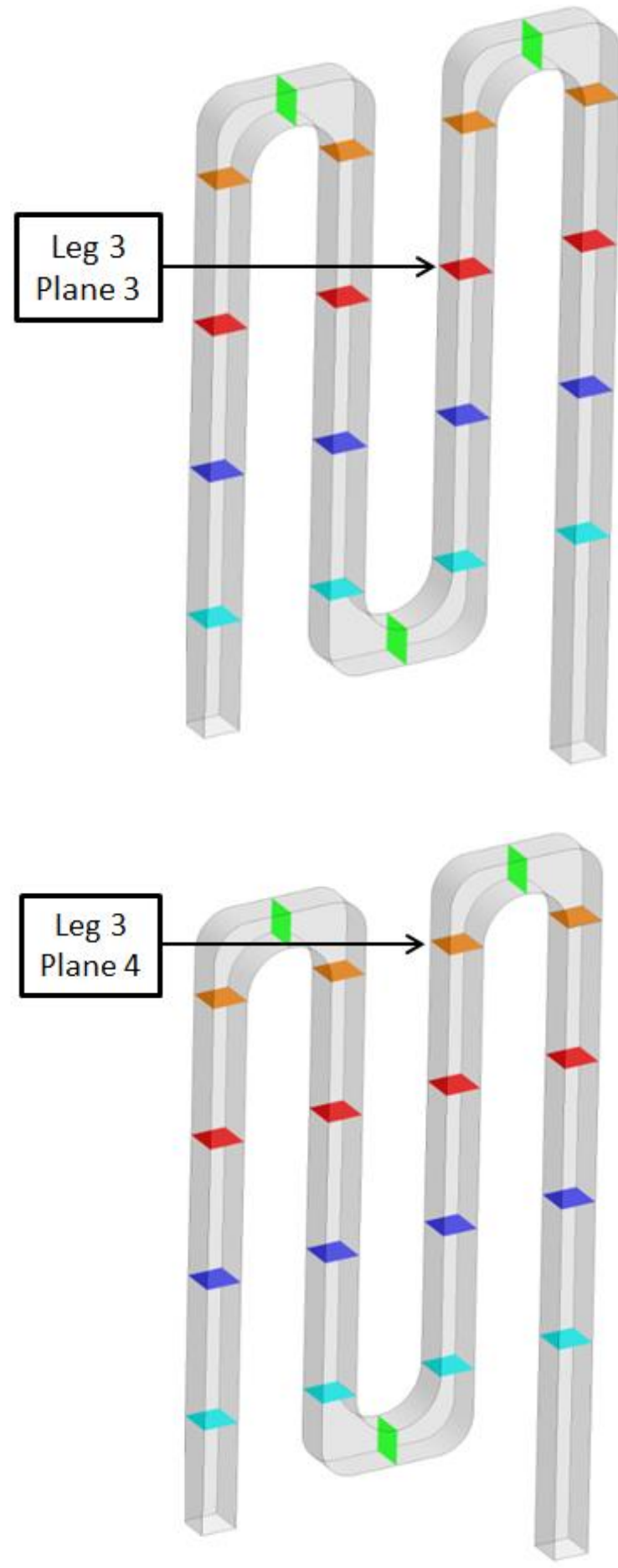

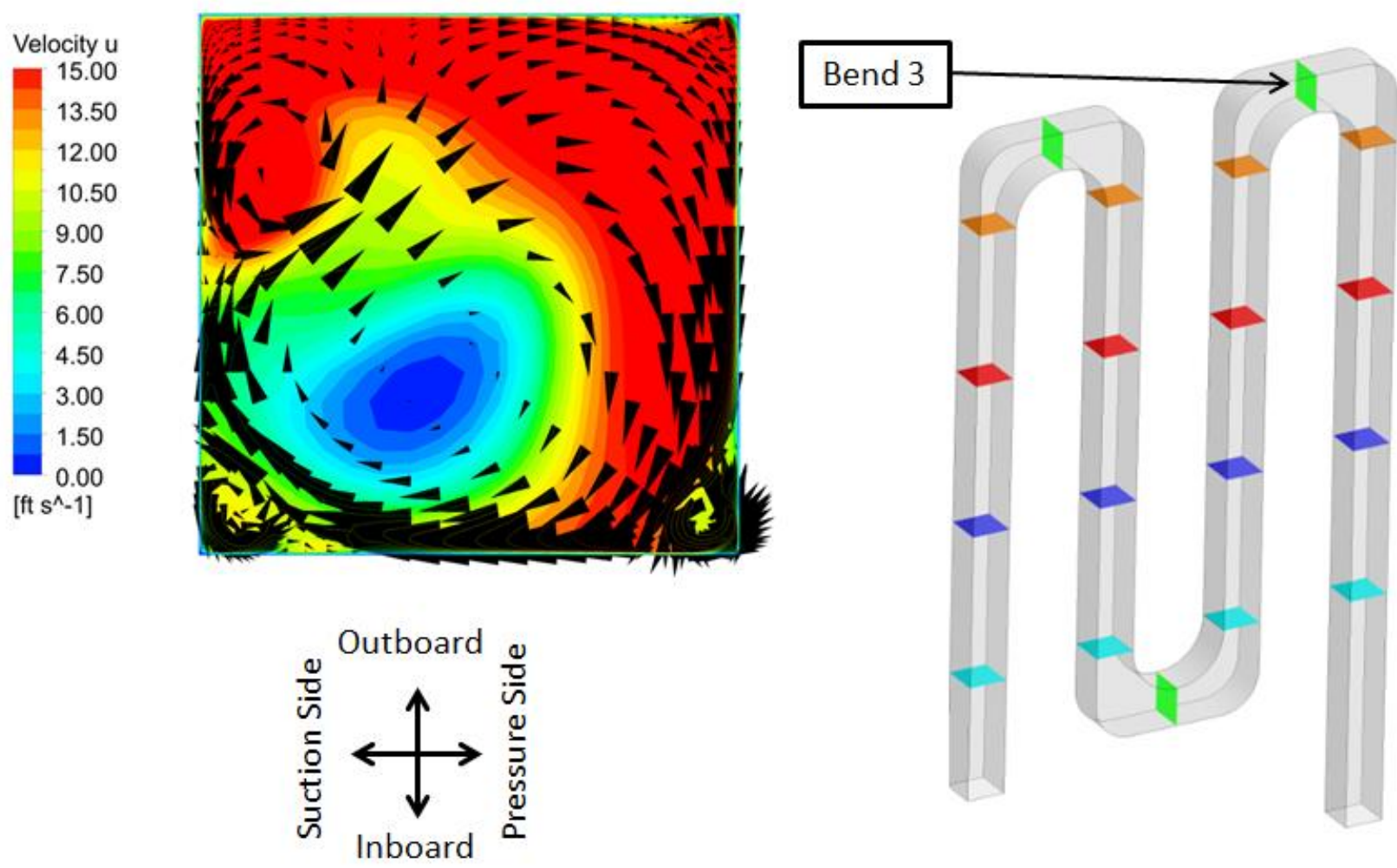

Case 6
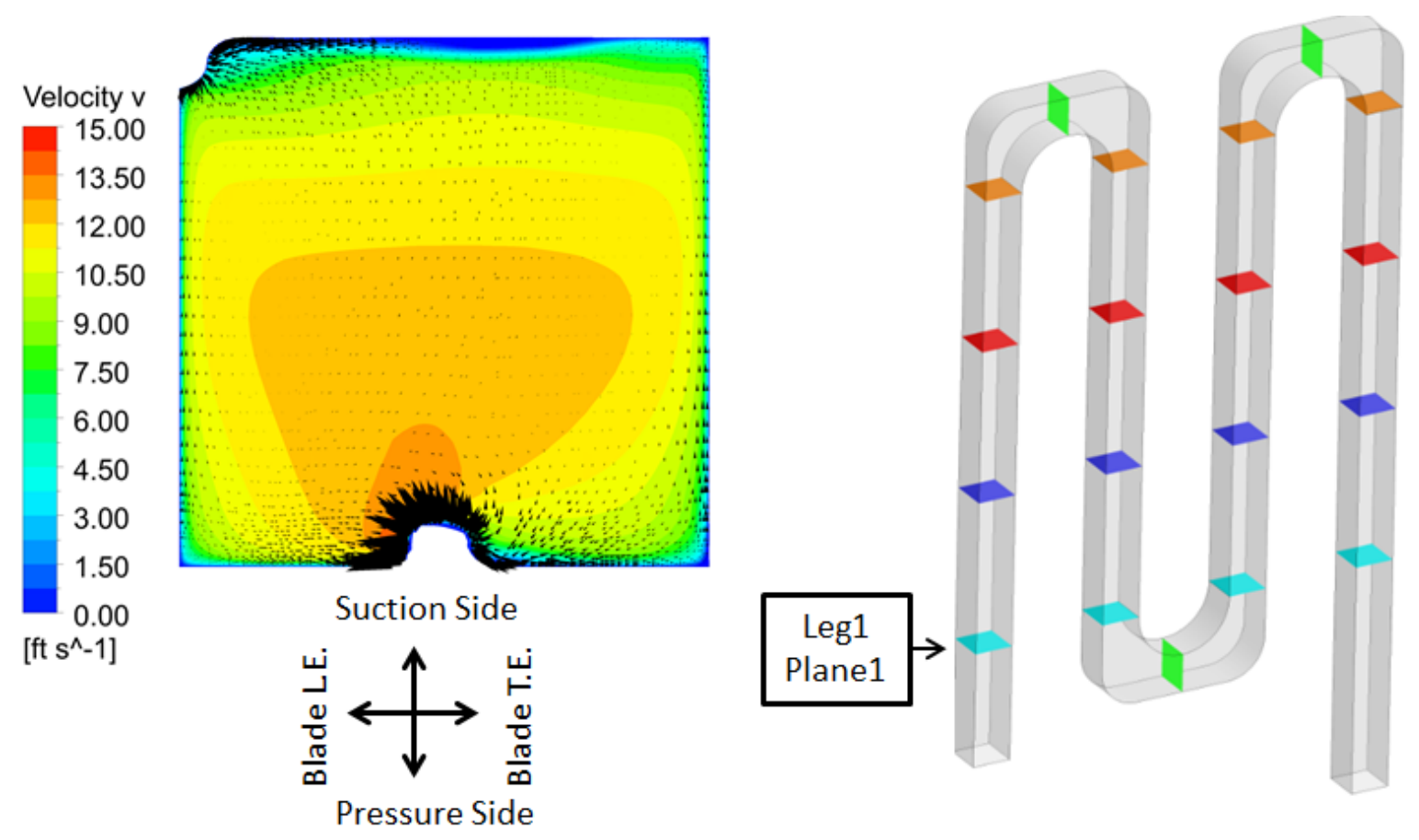


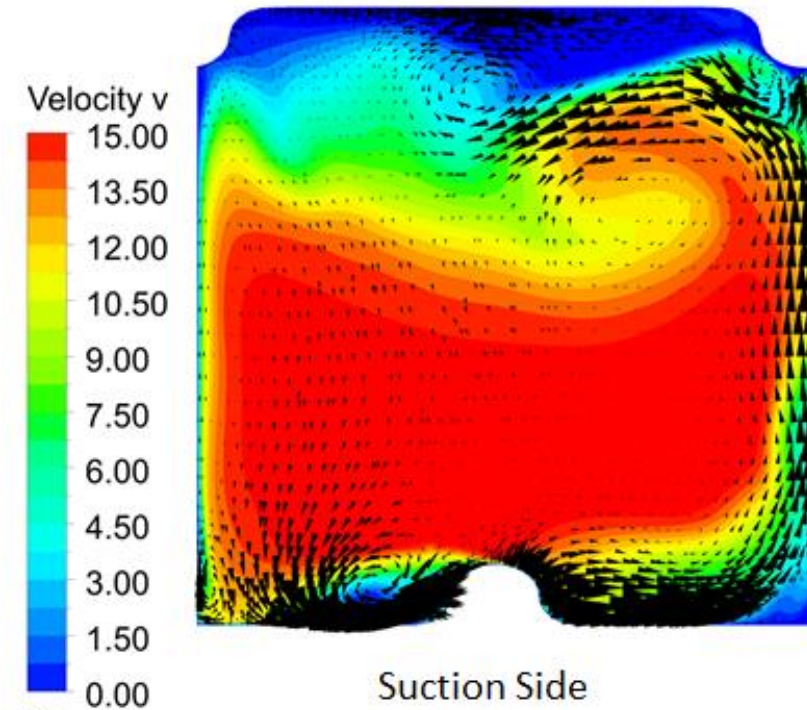

[ft s$\left.{ }^{\wedge}-1\right]$
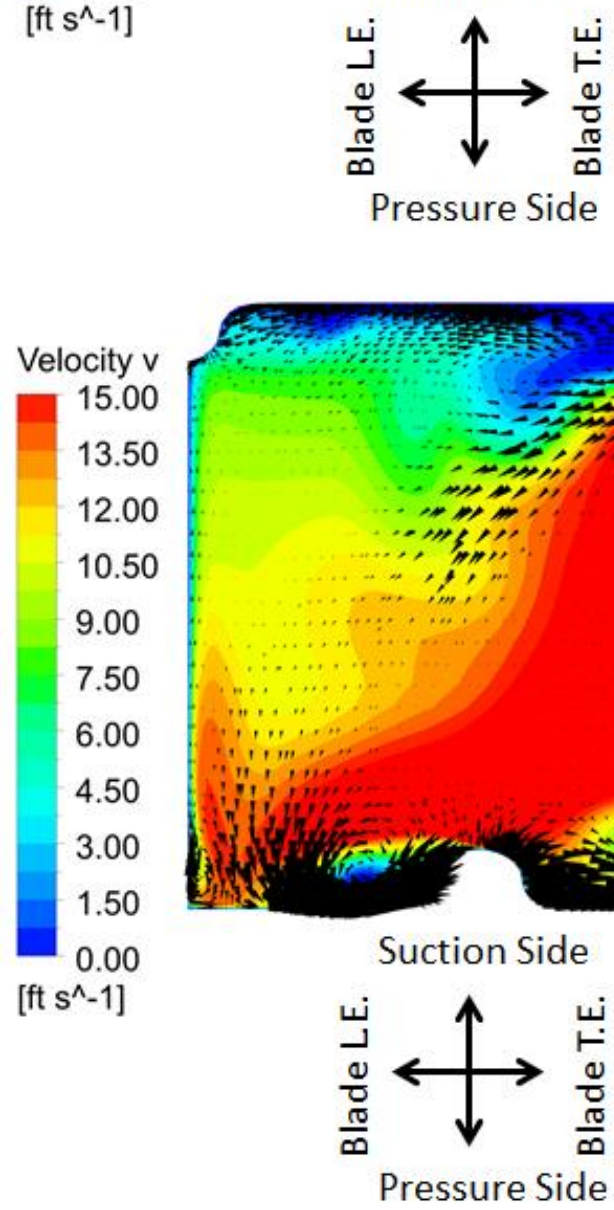
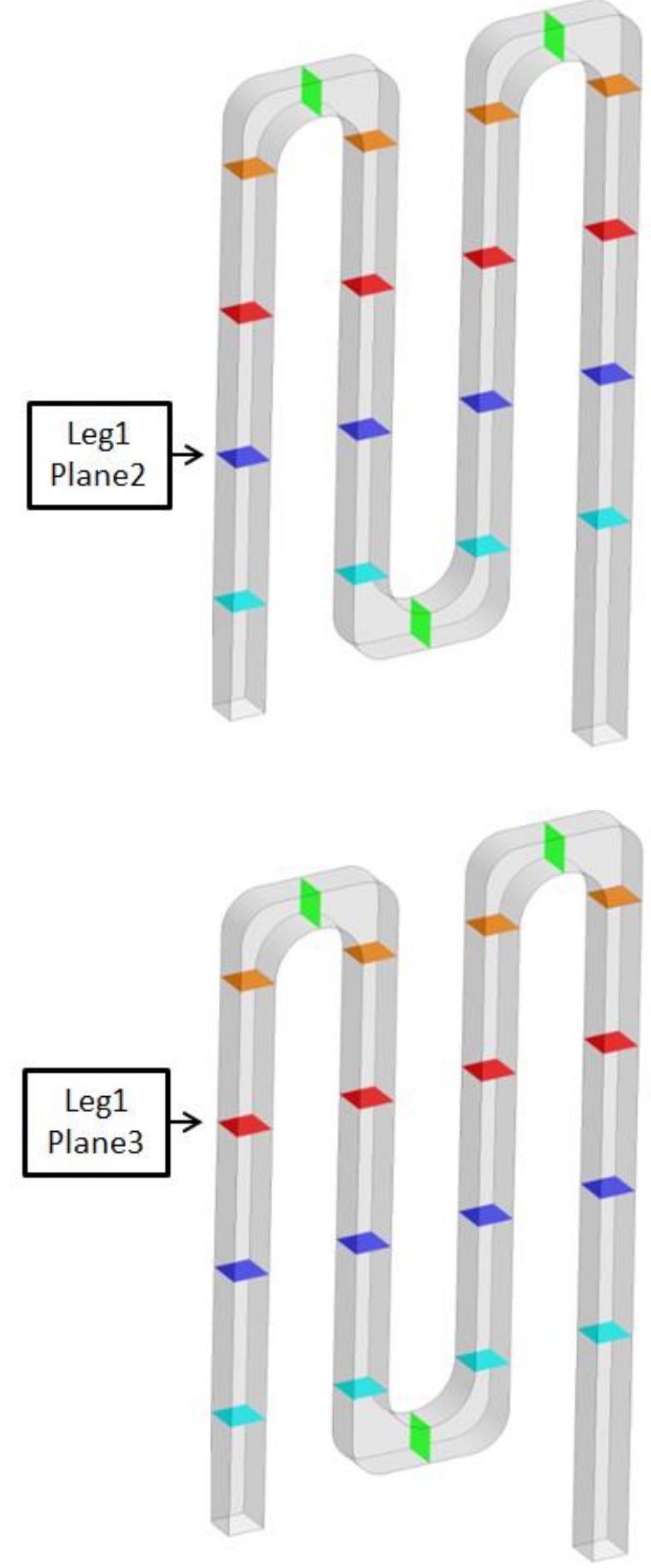

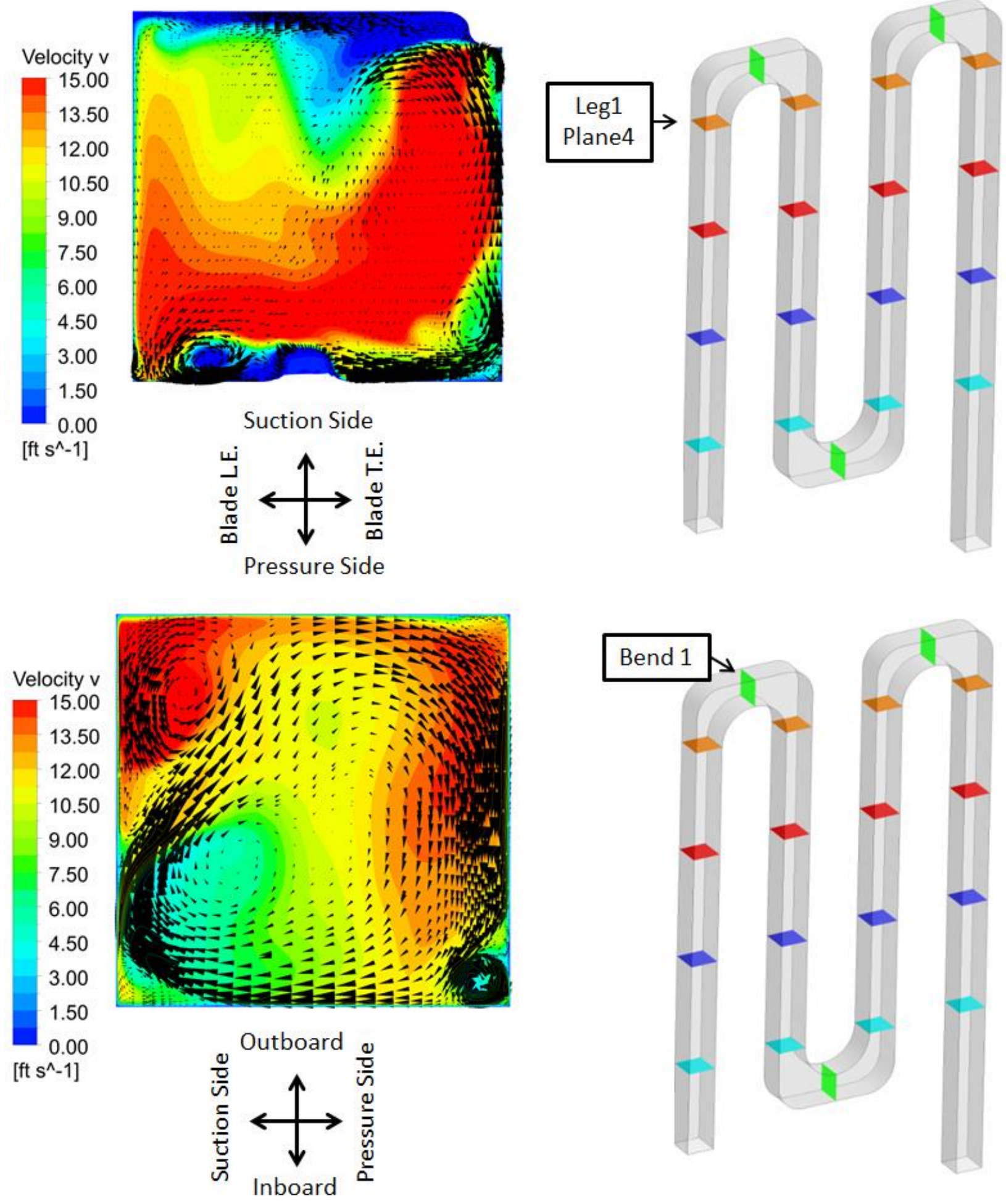

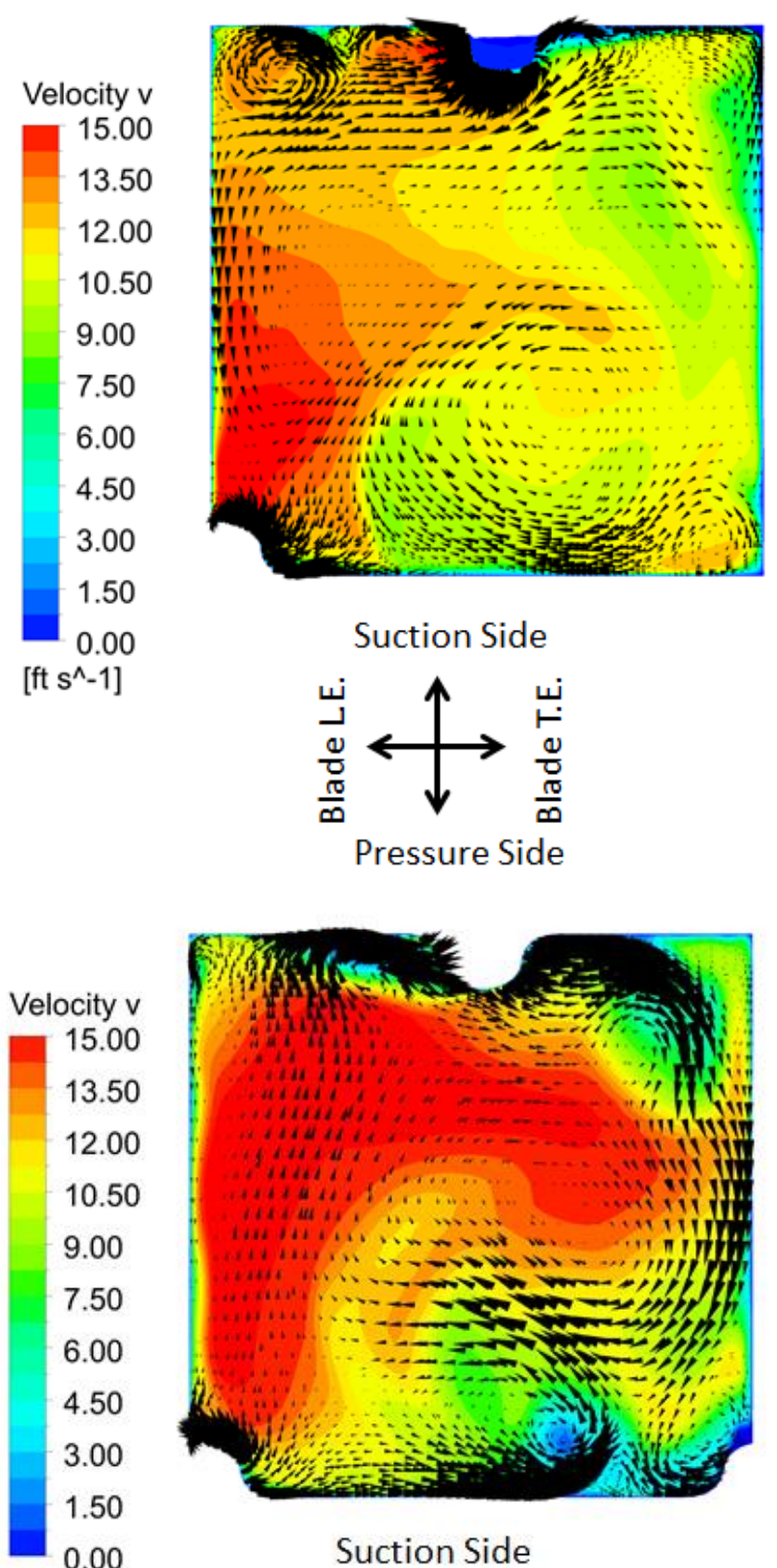

[ft s^-1]

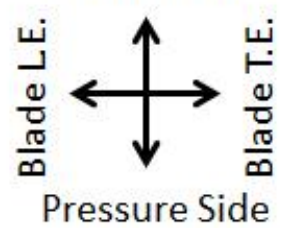

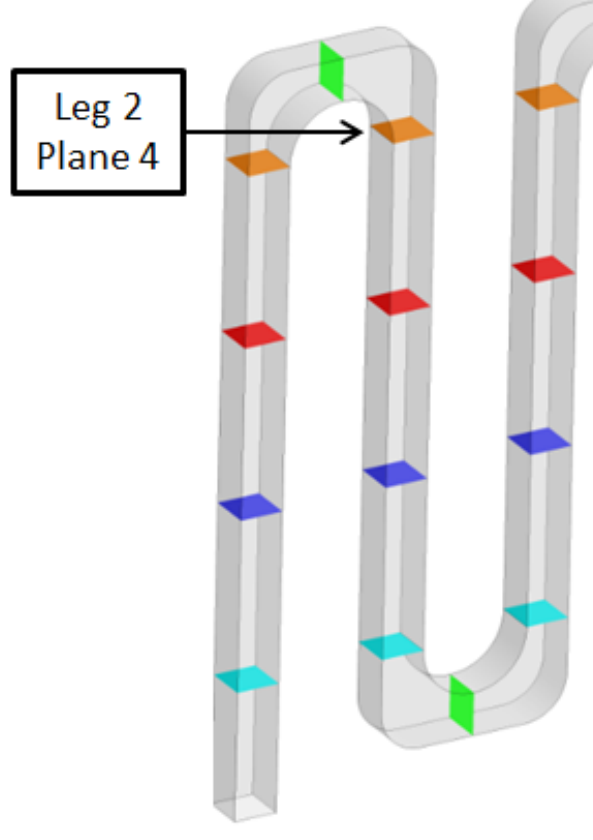

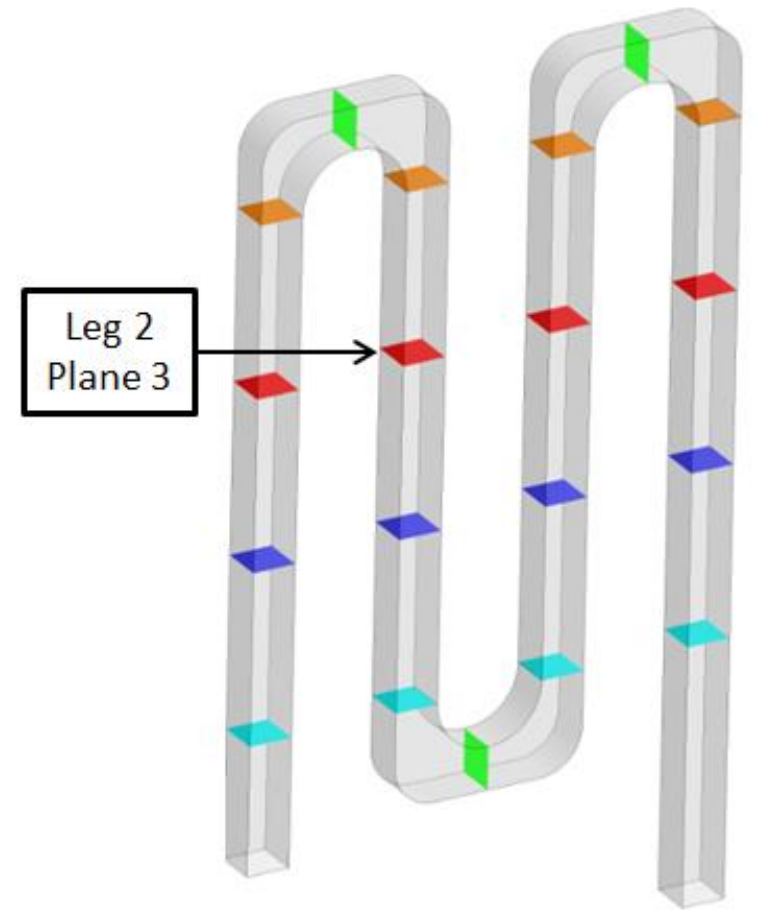



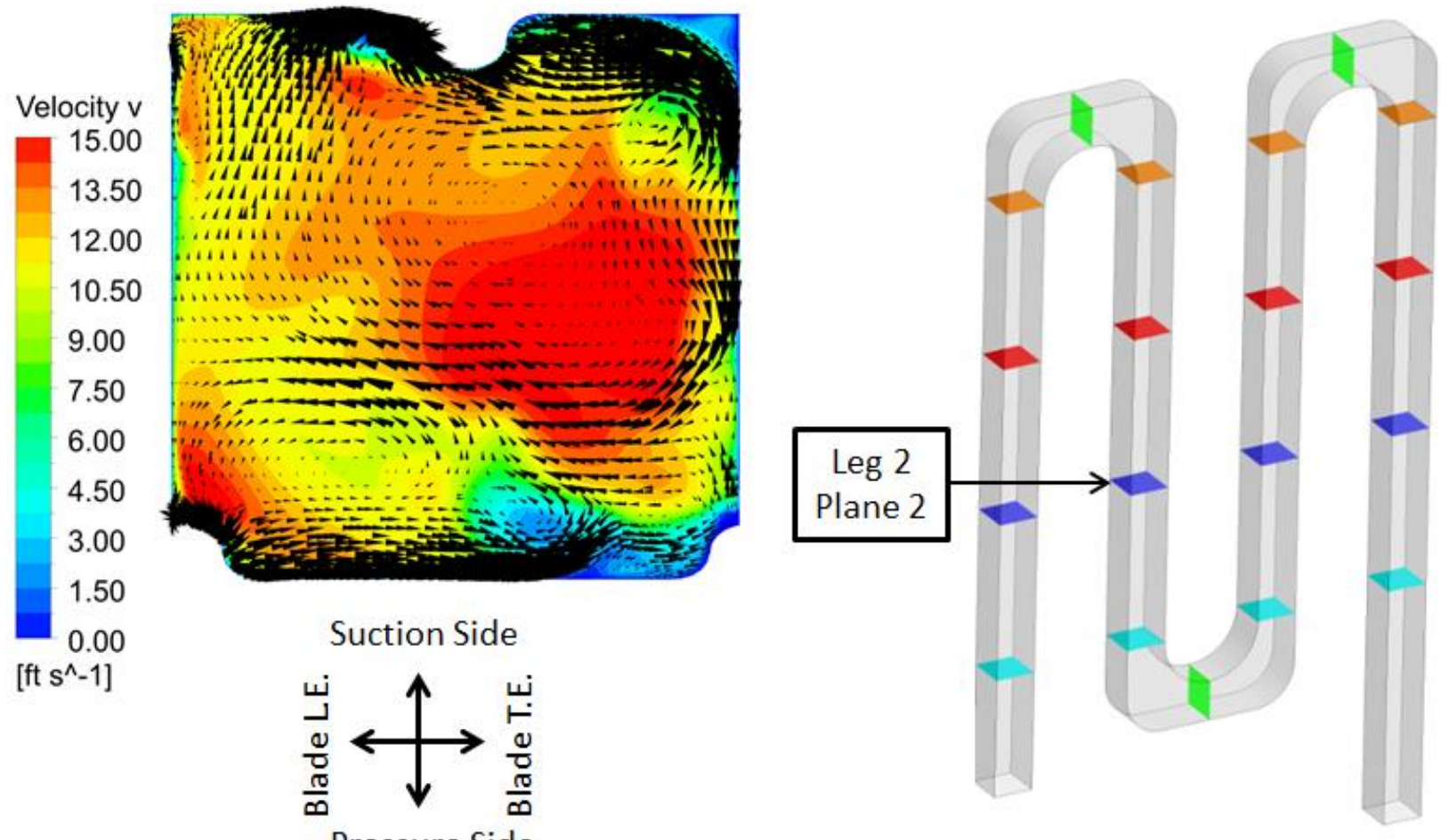

Suction Side
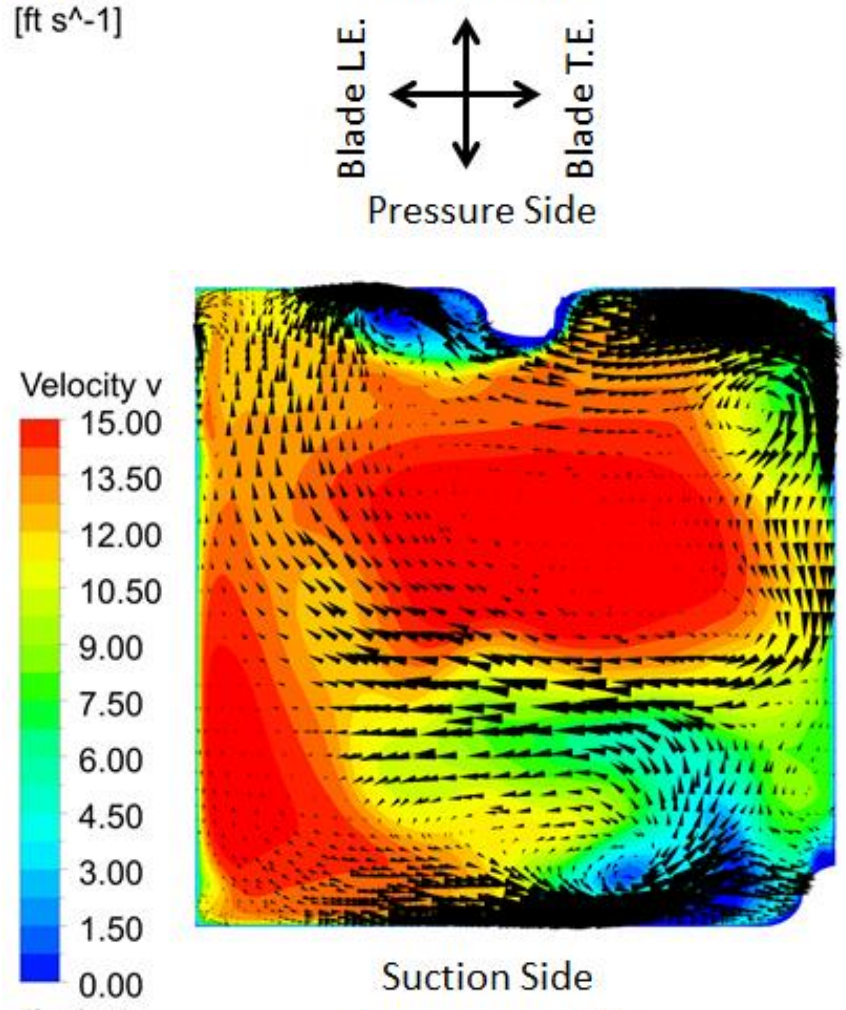

[ft s^-1]

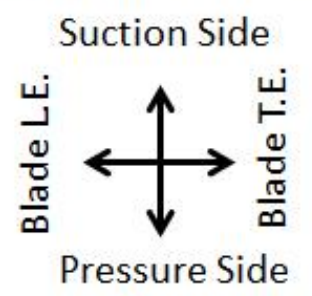

Leg 2

Plane 1 

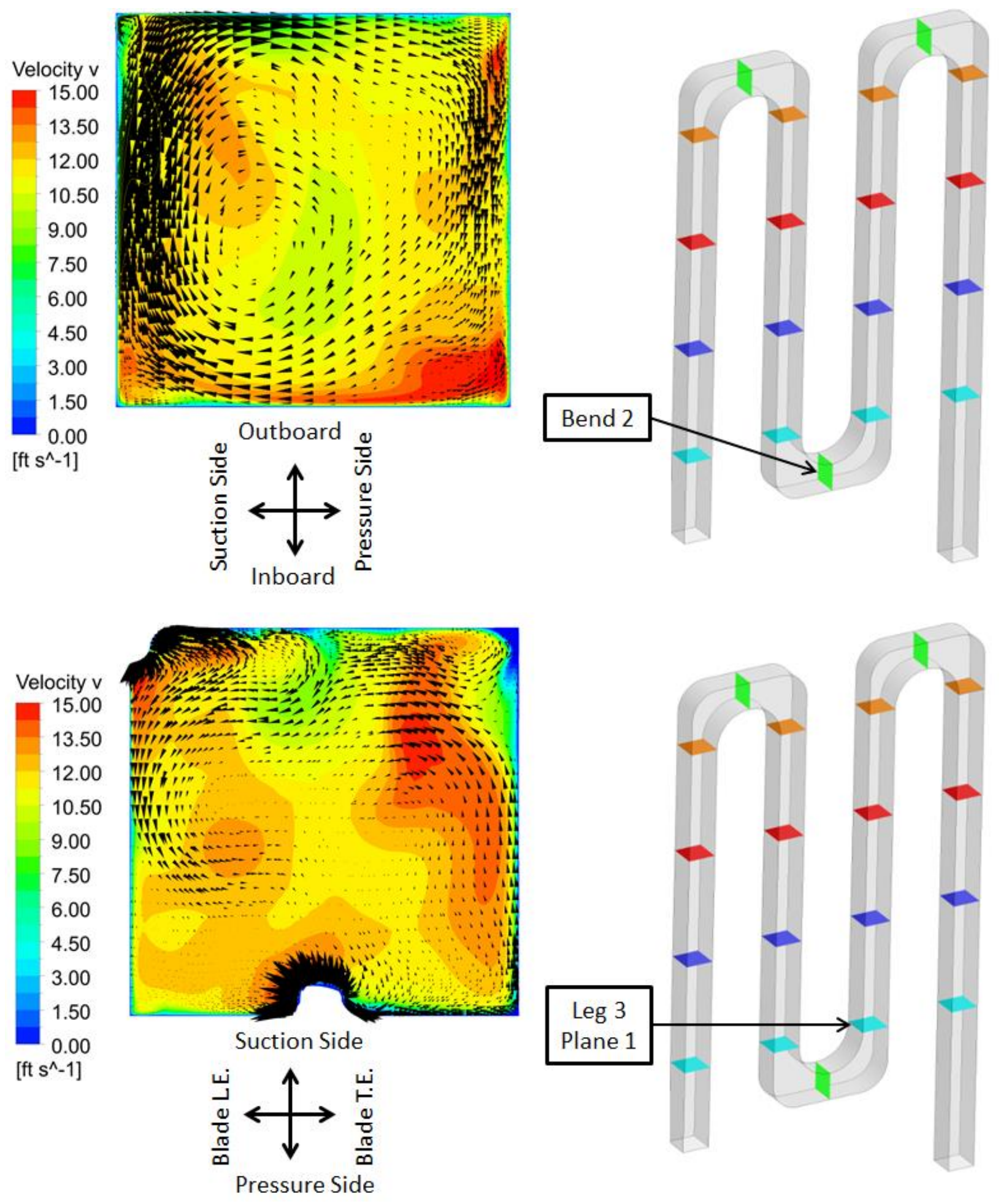


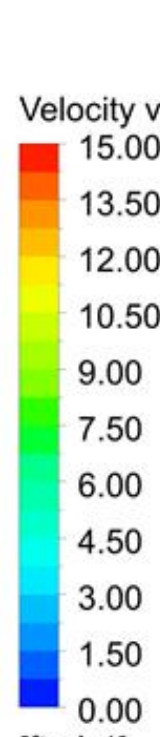

[ft s^-1]

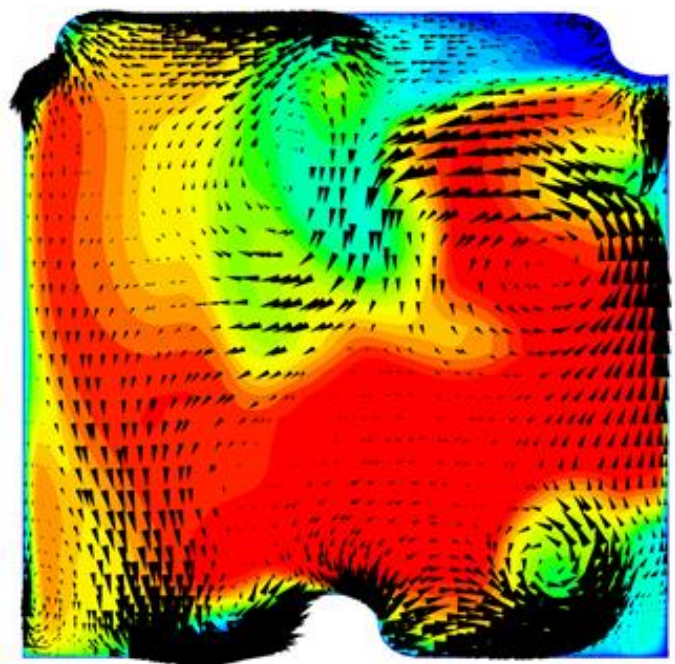

Suction Side
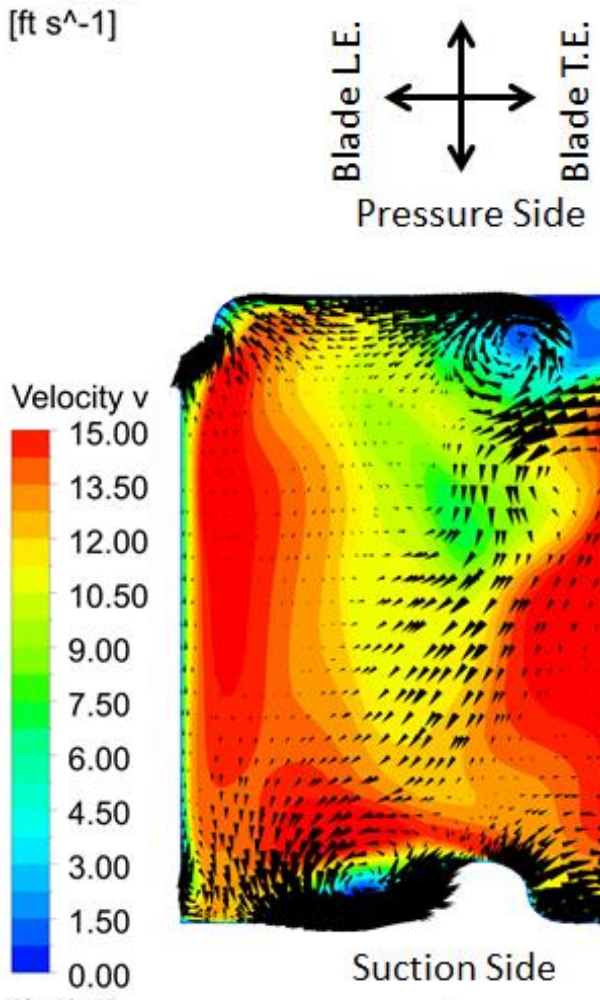

[ft $\mathrm{s}^{\wedge}-1$ ]
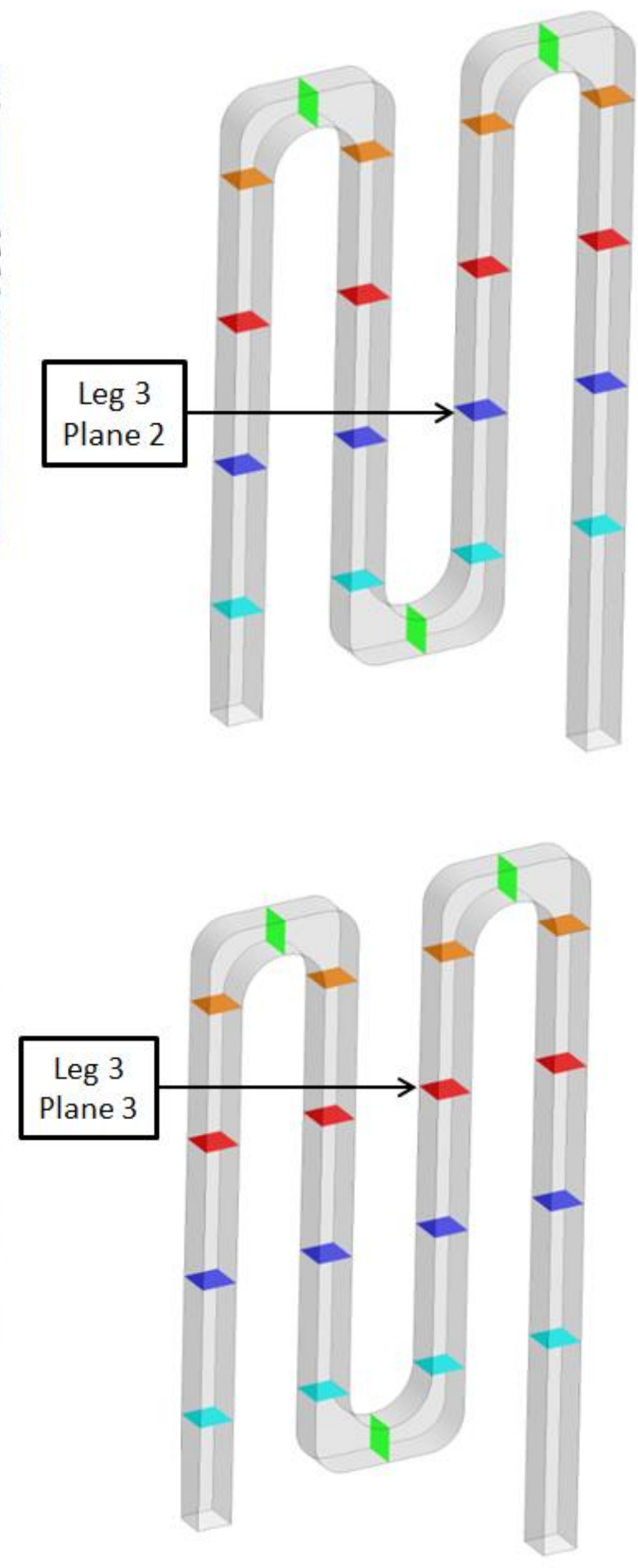

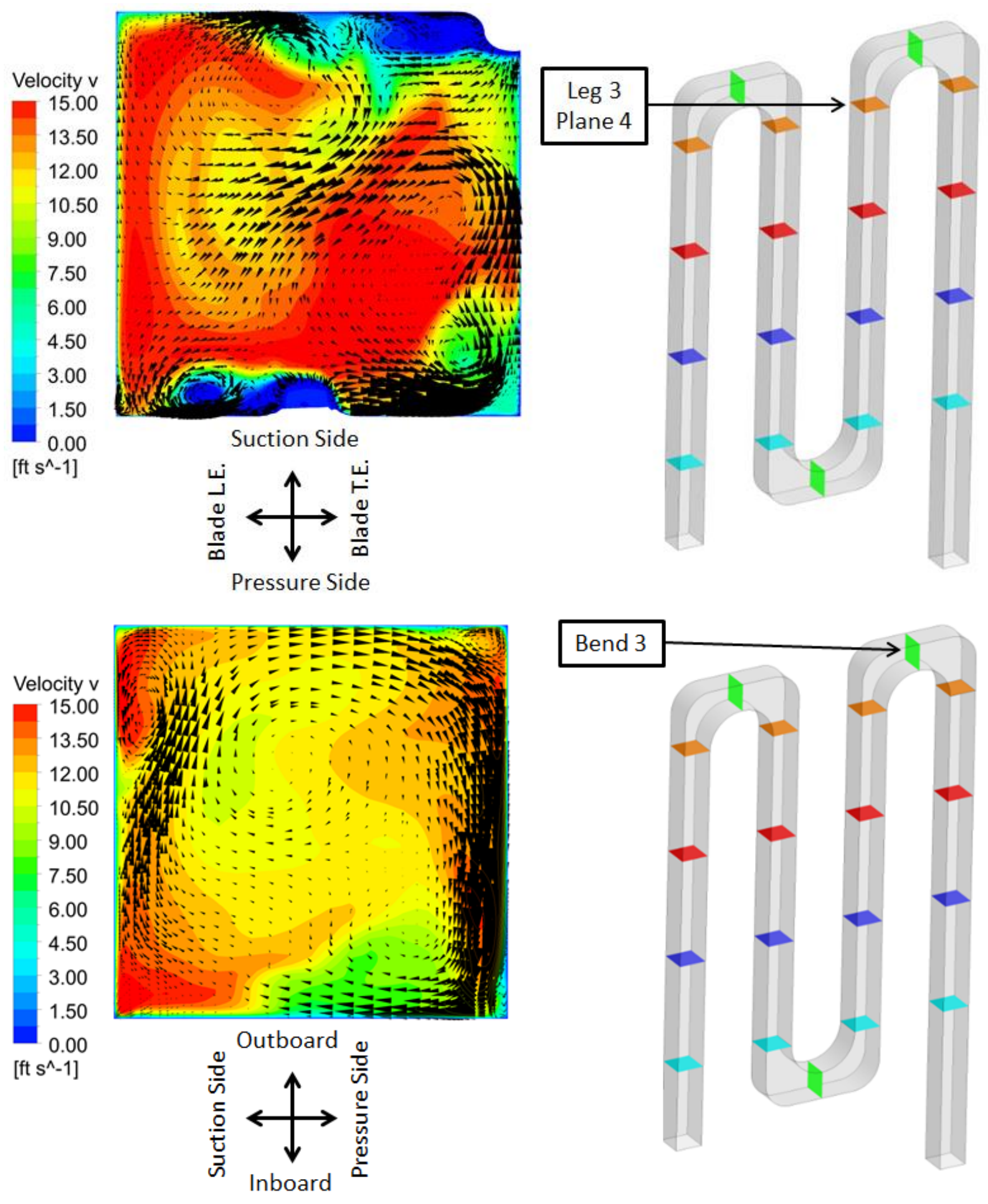

Case 7 

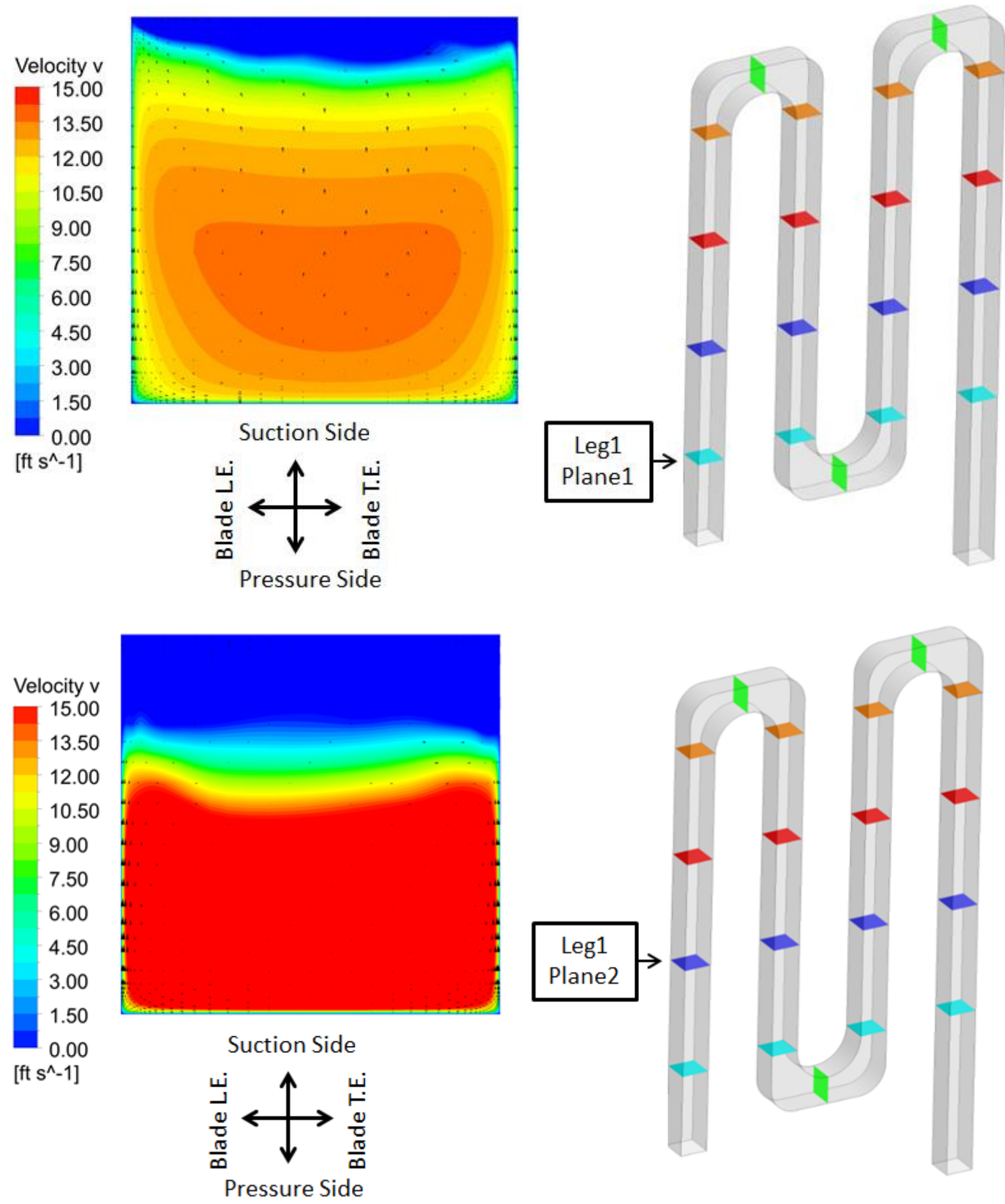

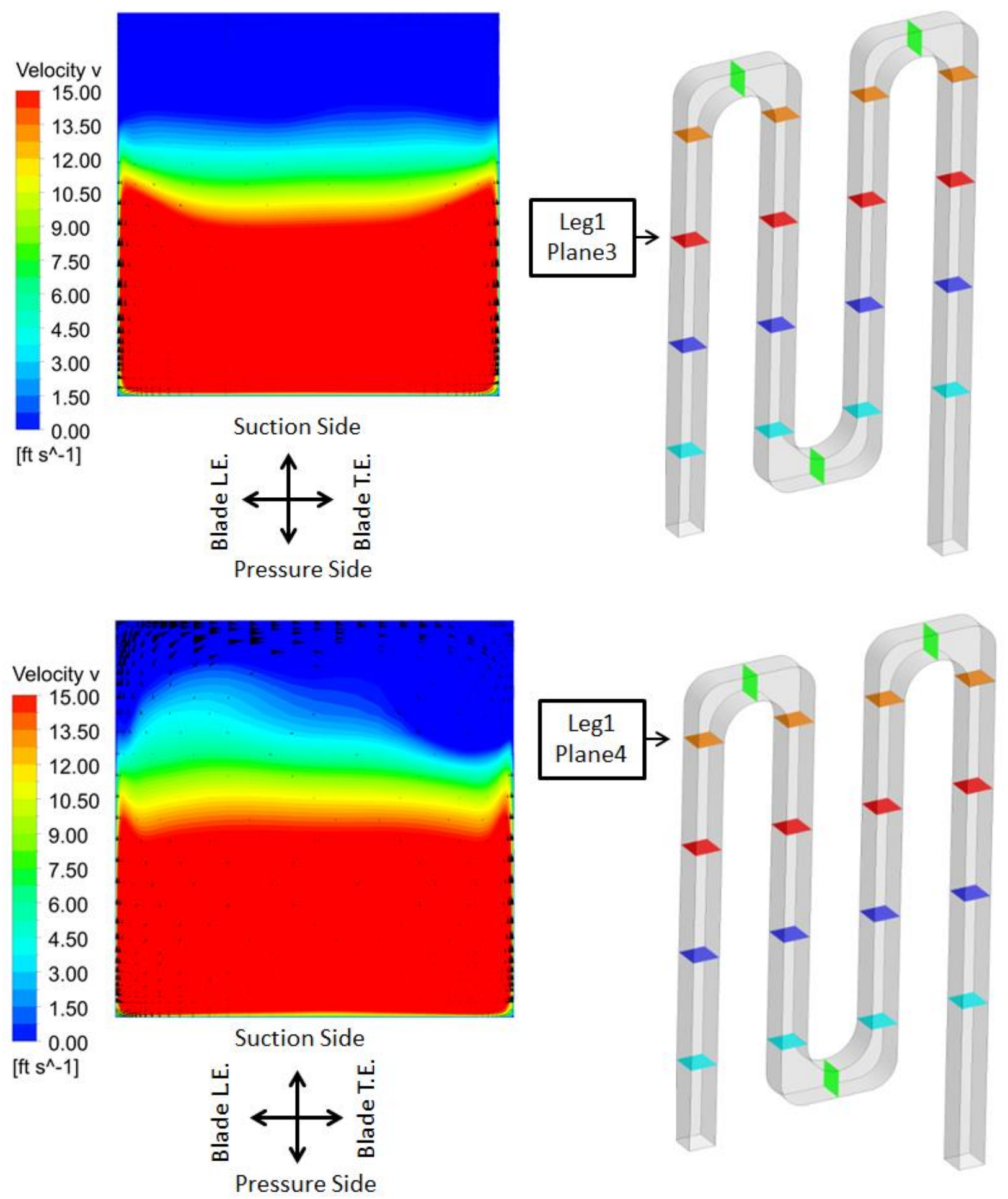

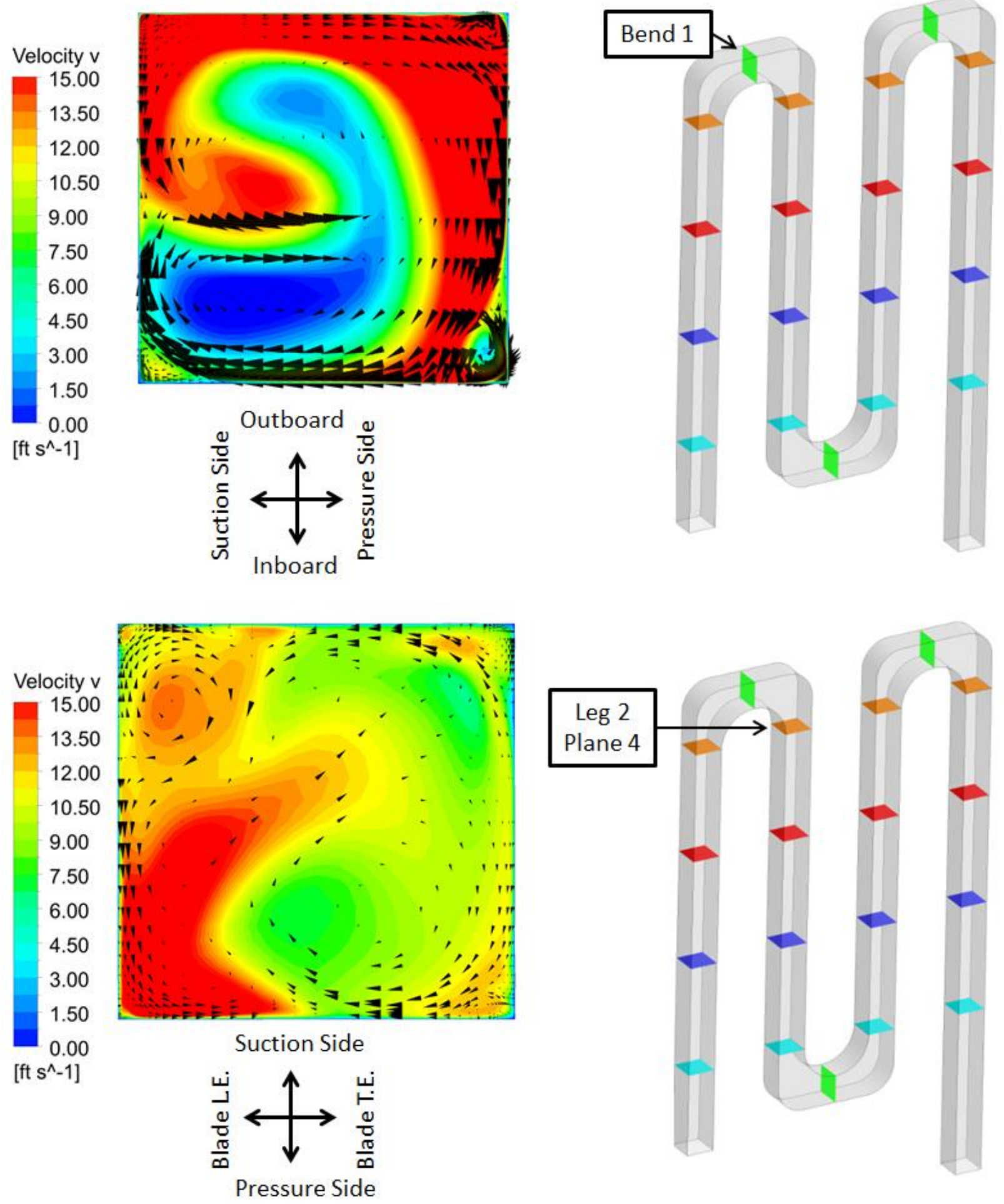

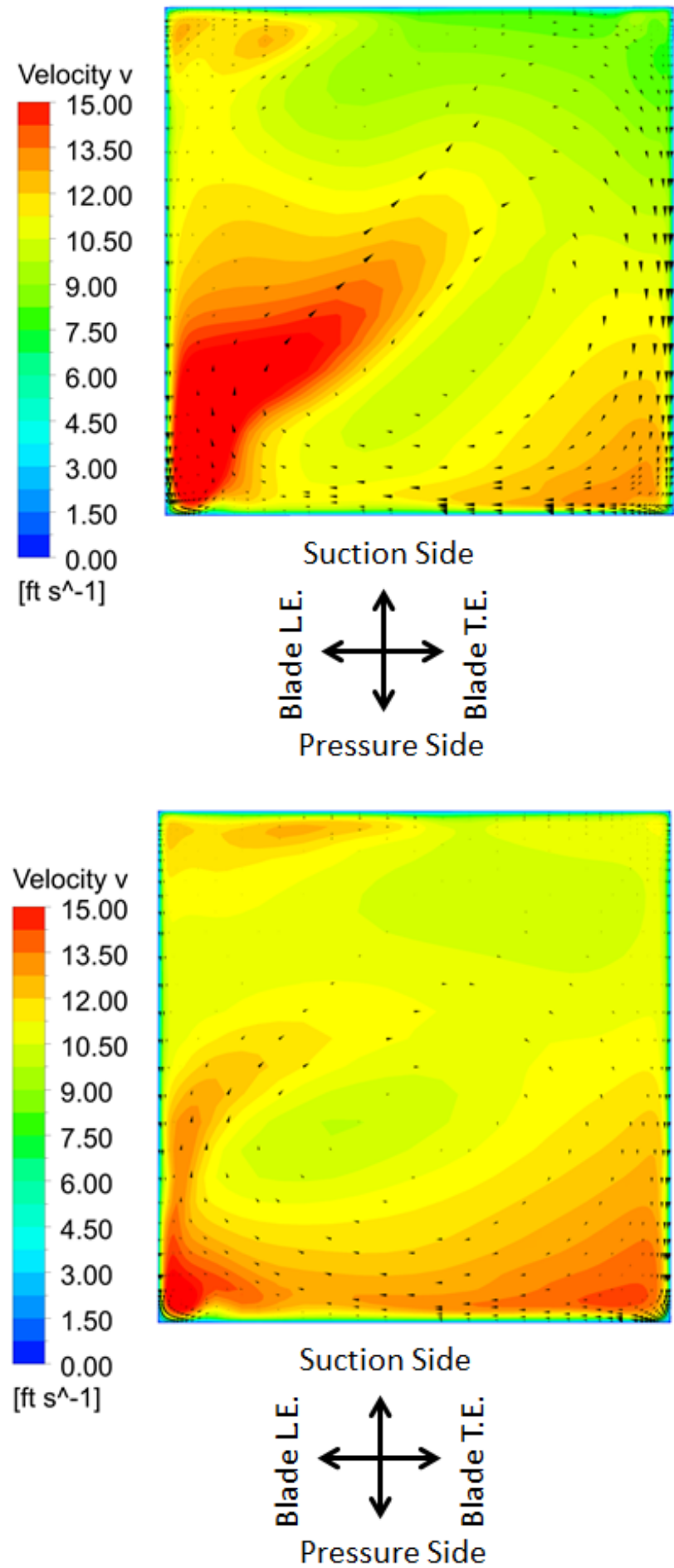

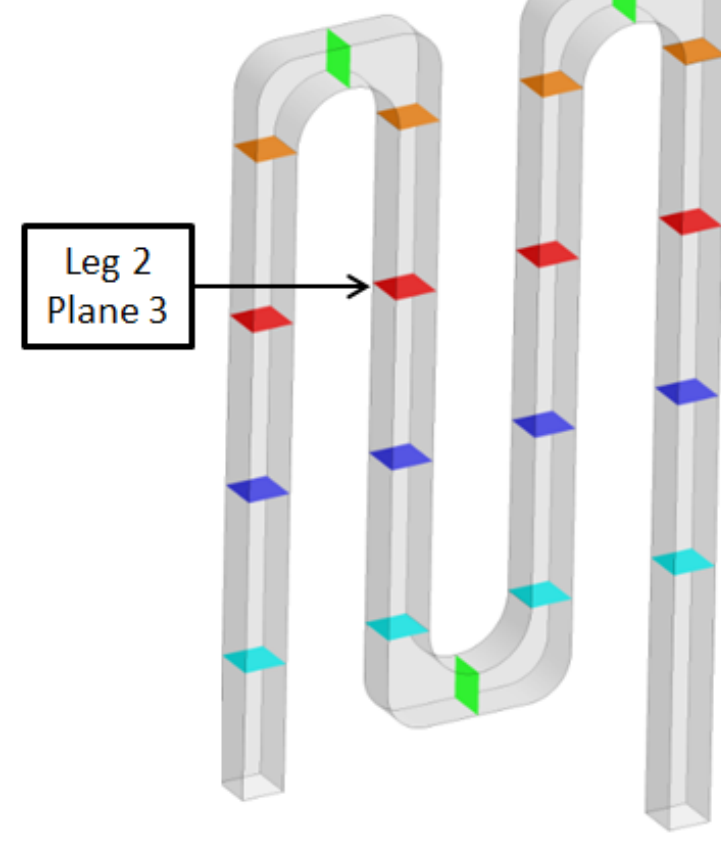

Leg 2 Plane 2 

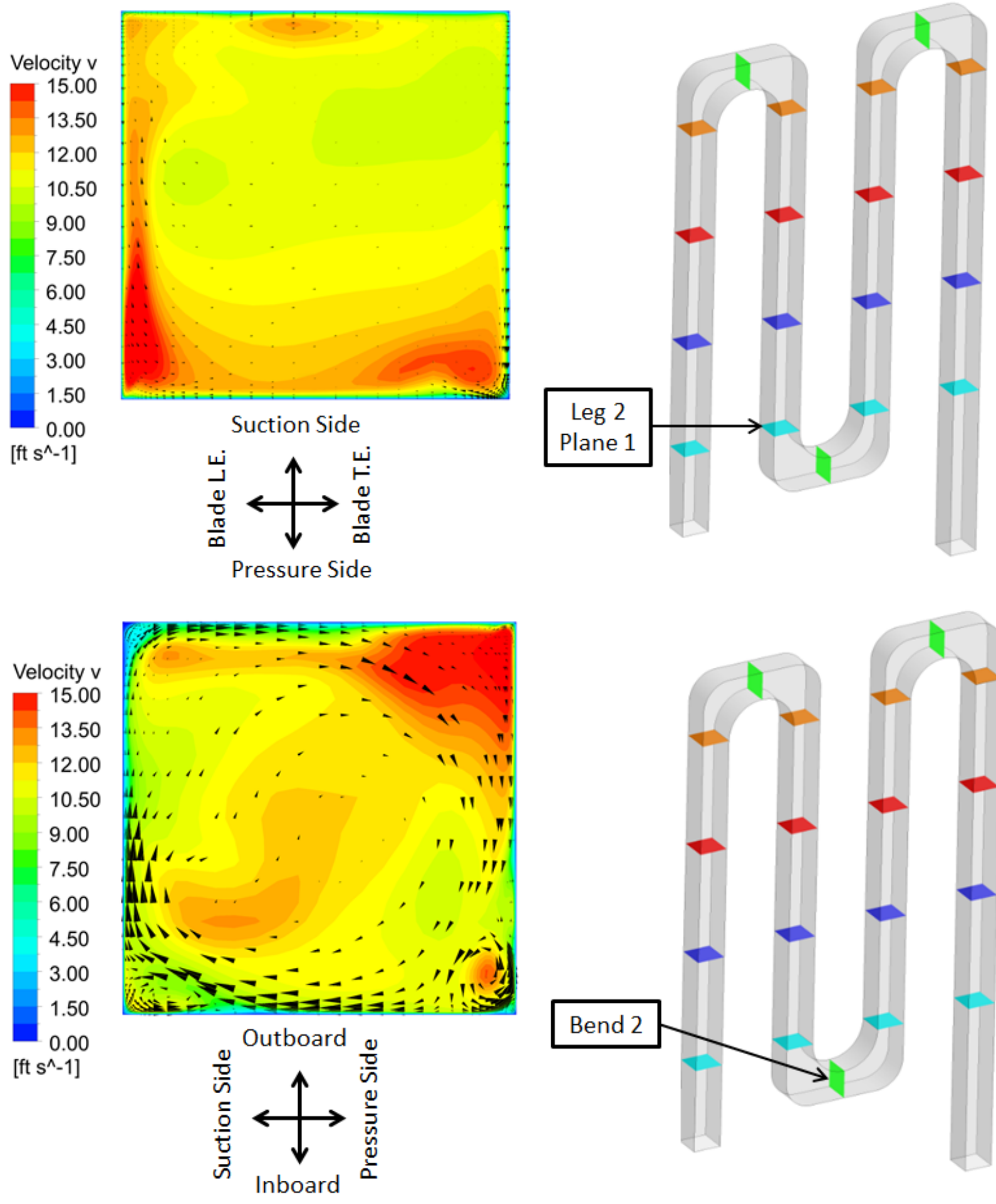

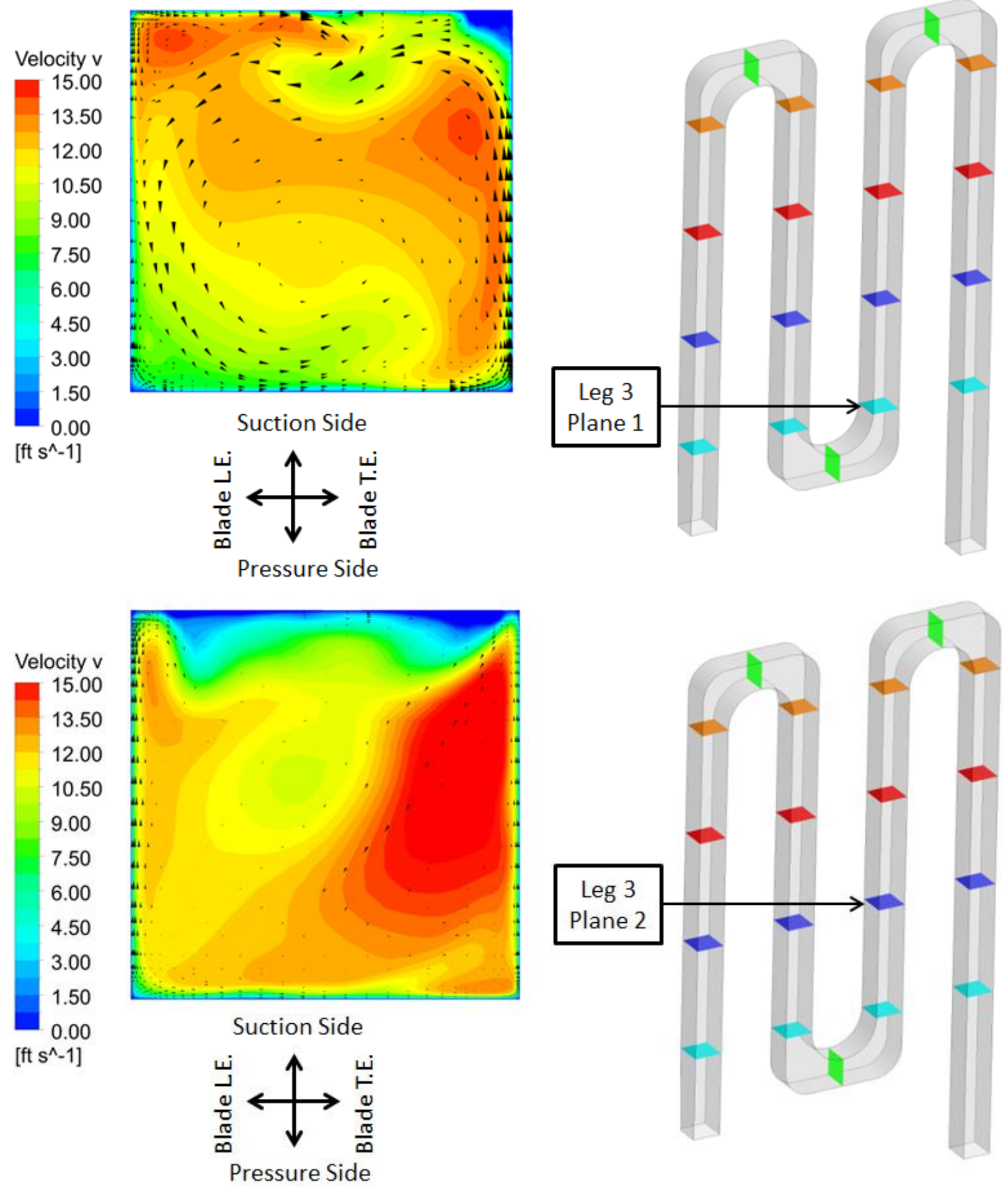

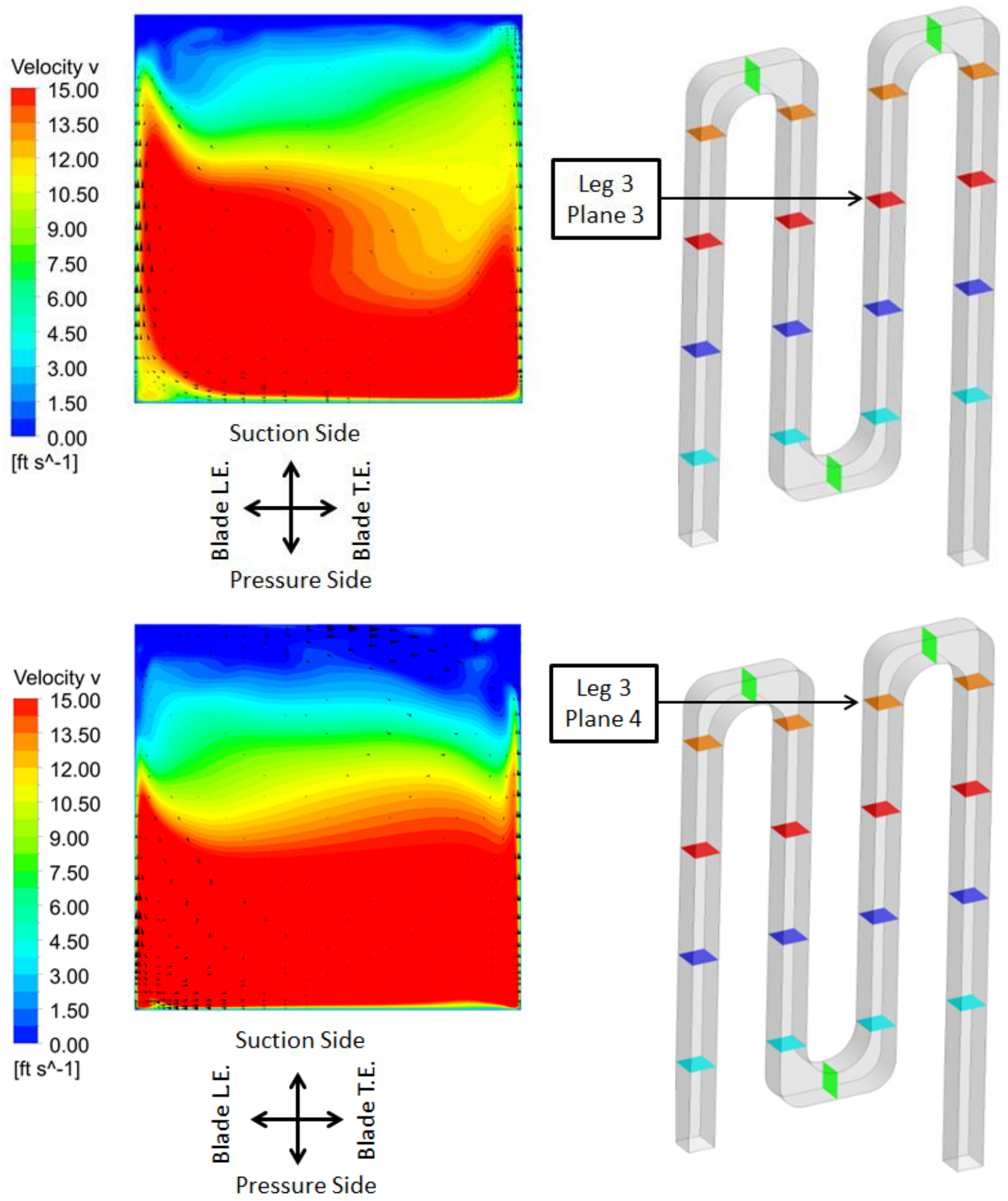

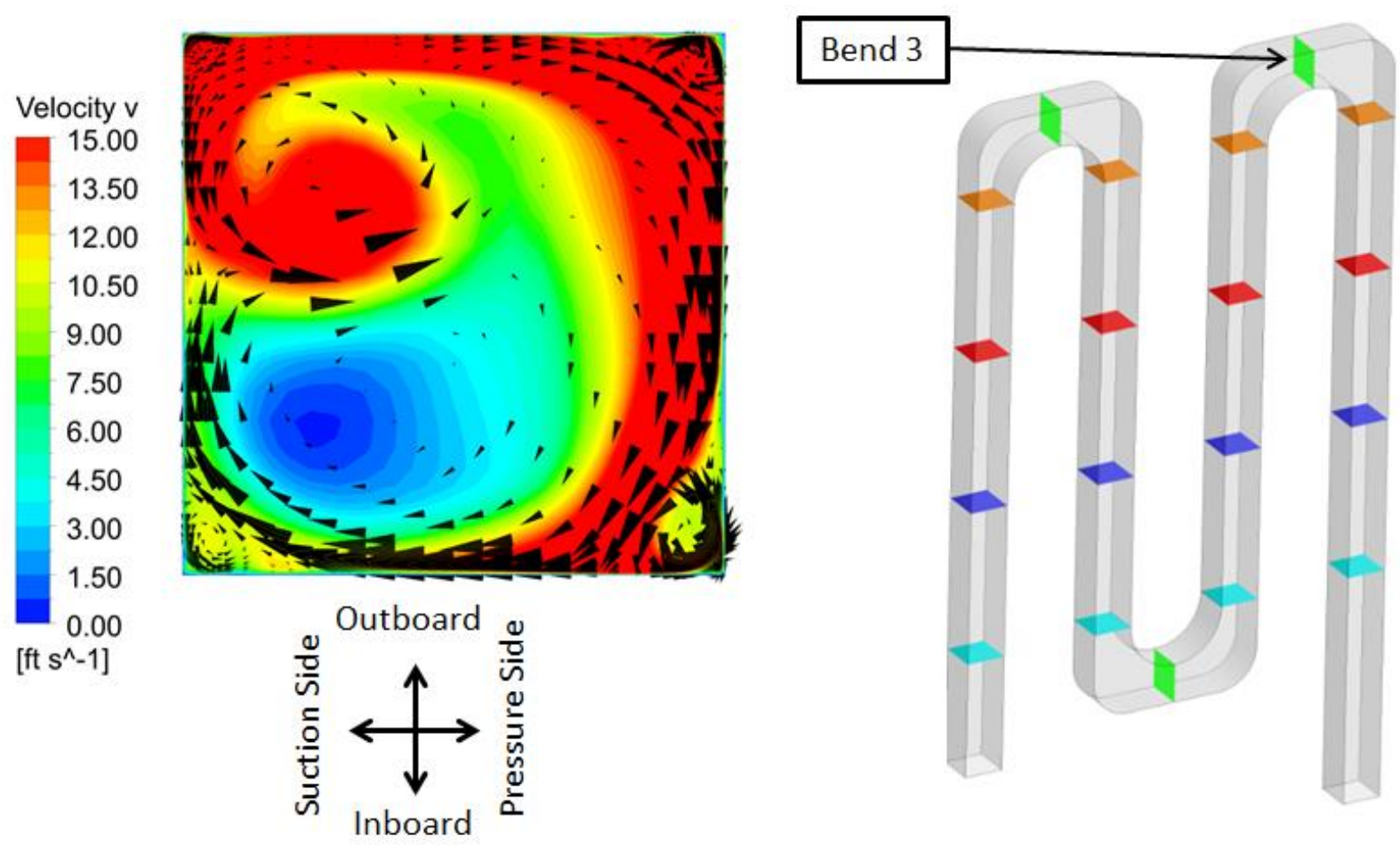